Prepared for the U.S. Department of Energy

under Contract DE-AC05-76RL01830

\title{
Evaluation of Conservation Voltage Reduction (CVR) on a National Level
}

$\begin{array}{ll}\text { KP Schneider } & \text { FK Tuffner } \\ \text { JC Fuller } & \text { R Singh }\end{array}$

July 2010

Pacific Northwest

NATIONAL LABORATORY

Proudly Operated by Battelle Since 1965 


\title{
DISCLAIMER
}

This report was prepared as an account of work sponsored by an agency of the United States Government. Neither the United States Government nor any agency thereof, nor Battelle Memorial Institute, nor any of their employees, makes any warranty, express or implied, or assumes any legal liability or responsibility for the accuracy, completeness, or usefulness of any information, apparatus, product, or process disclosed, or represents that its use would not infringe privately owned rights. Reference herein to any specific commercial product, process, or service by trade name, trademark, manufacturer, or otherwise does not necessarily constitute or imply its endorsement, recommendation, or favoring by the United States Government or any agency thereof, or Battelle Memorial Institute. The views and opinions of authors expressed herein do not necessarily state or reflect those of the United States Government or any agency thereof.

\author{
PACIFIC NORTHWEST NATIONAL LABORATORY \\ operated by \\ BATTELLE \\ for the \\ UNITED STATES DEPARTMENT OF ENERGY \\ under Contract DE-AC05-76RL01830
}

Printed in the United States of America

Available to DOE and DOE contractors from the Office of Scientific and Technical Information, P.O. Box 62, Oak Ridge, TN 37831-0062; ph: (865) 576-8401 fax: (865) 576-5728 email:

reports@adonis.osti.gov

\author{
Available to the public from the National Technical Information Service, \\ U.S. Department of Commerce, 5285 Port Royal Rd., Springfield, VA 22161 \\ ph: (800) 553-6847 fax: (703) 605-6900 \\ email: orders@ntis.fedworld.gov online ordering: http://www.ntis.gov/ordering.htm
}

This document was printed on recycled paper. $(9 / 2003)$ 


\section{Table of Contents}

TABLE OF FIGURE

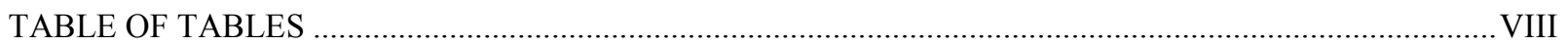

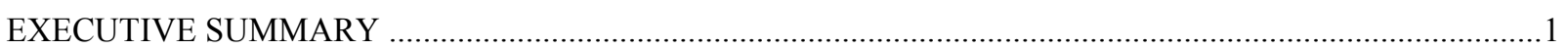

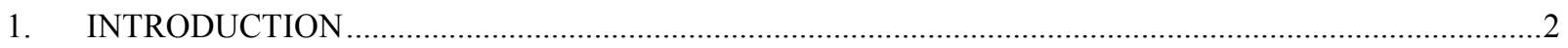

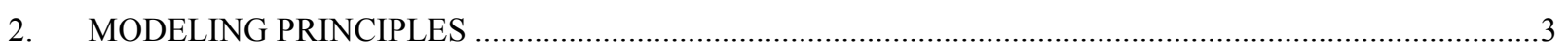

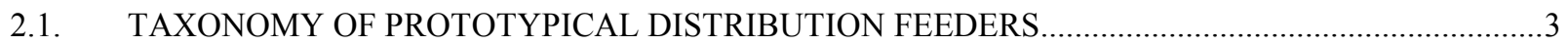

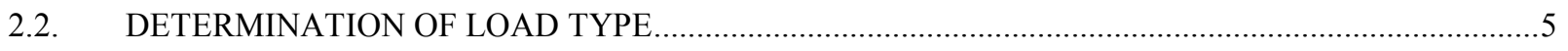

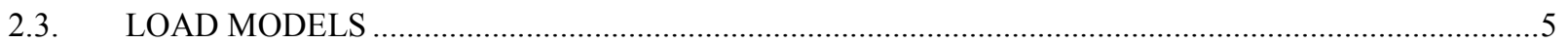

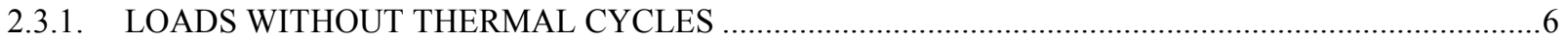

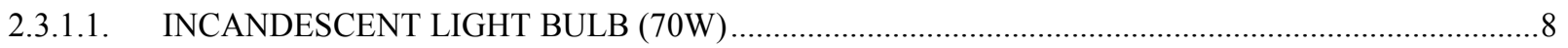

2.3.1.2. MAGNAVOX TELEVISION (CATHODE RAY TUBE) ................................................................

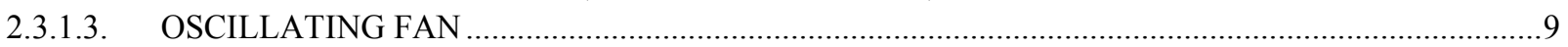

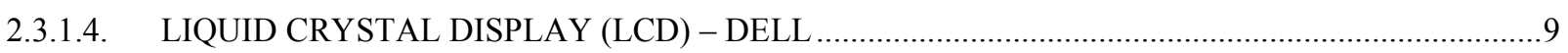

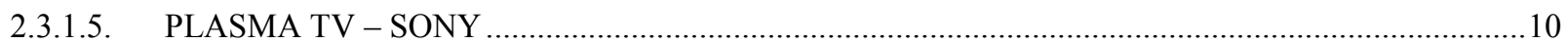

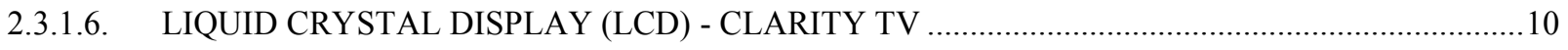

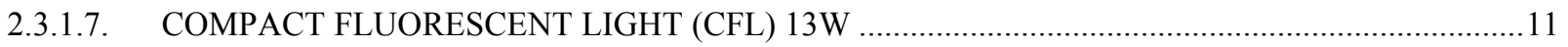

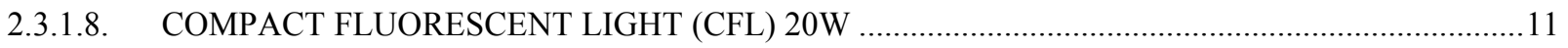

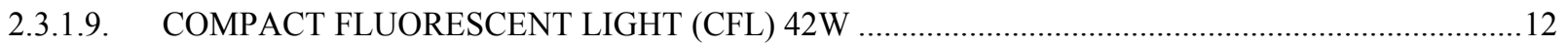

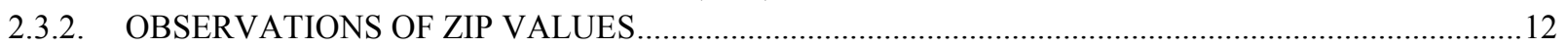

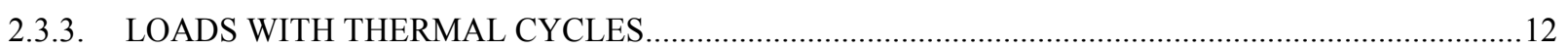

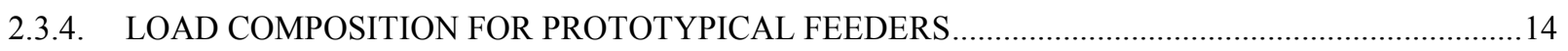

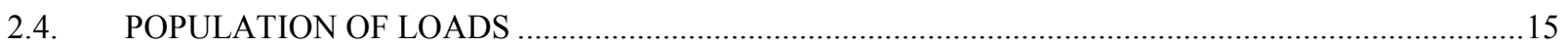

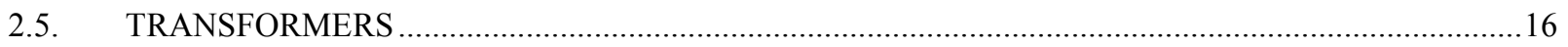

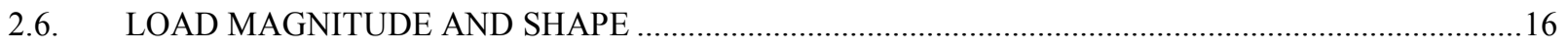

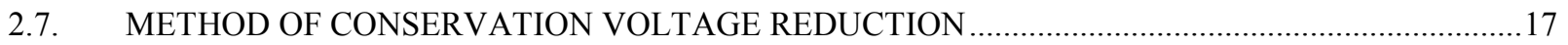

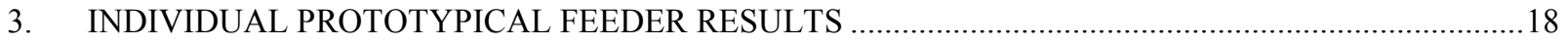

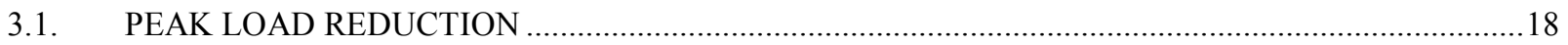

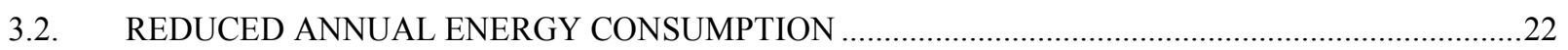

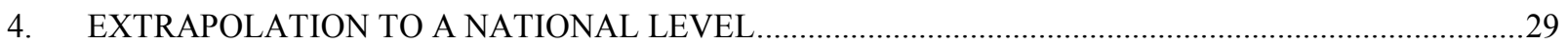

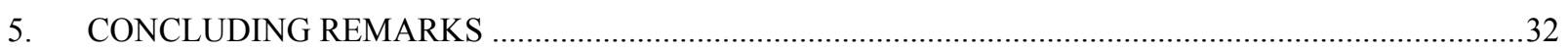

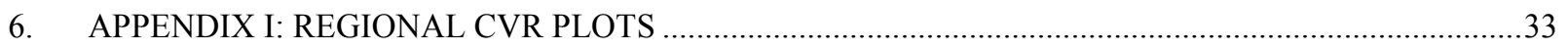

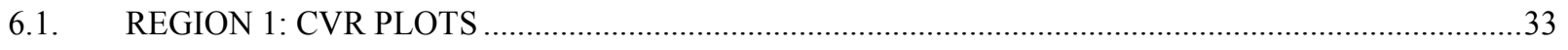

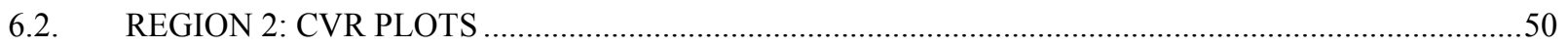

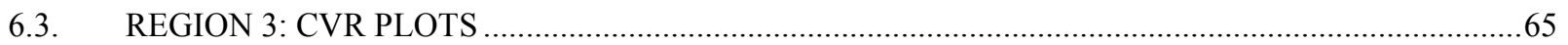

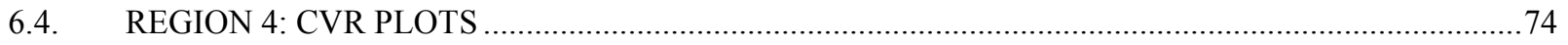

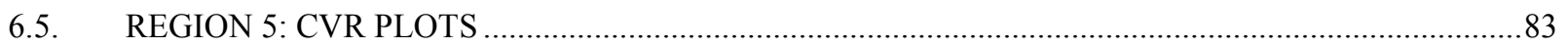

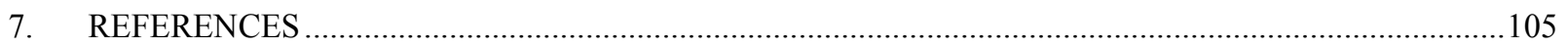




\section{Table of Figure}

Figure 2.1: Climate Zones Used for Development of Prototypical Feeders.......................................... 3

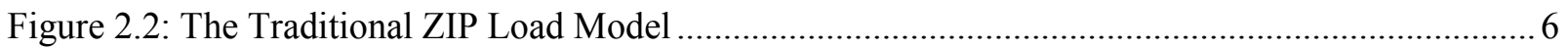

Figure 2.3: Voltage Dependent Energy Consumption of a 70W Incandescent Light Bulb.................... 8

Figure 2.4: Voltage Dependent Energy Consumption of a CRT Television ......................................... 8

Figure 2.5: Voltage Dependent Energy Consumption of an Oscillating Fan ......................................... 9

Figure 2.6: Voltage Dependent Energy Consumption of a Dell LCD ................................................. 9

Figure 2.7: Voltage Dependent Energy Consumption of a Sony Plasma ................................................ 10

Figure 2.8: Voltage Dependent Energy Consumption of a Clarity LCD ............................................. 10

Figure 2.9: Voltage Dependent Energy Consumption of a 13W CFL ................................................. 11

Figure 2.10: Voltage Dependent Energy Consumption of a 20W CFL ................................................ 11

Figure 2.11: Voltage Dependent Energy Consumption of a 42W CFL ................................................. 12

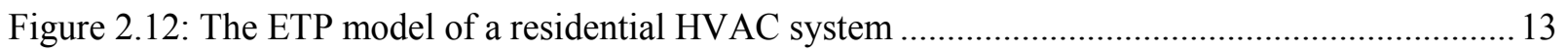

Figure 3.1: Peak Demand Change (kWh) by Taxonomy Feeder........................................................ 19

Figure 3.2: Peak Demand Change (\%) by Taxonomy Feeder ........................................................... 19

Figure 3.3: Peak loading (MW) by Taxonomy Feeder ..................................................................... 20

Figure 3.4: Minimum Annual EOL Voltages for Prototypical Feeders with CVR Off......................... 21

Figure 3.5: Minimum Annual EOL Voltages for Prototypical Feeders with CVR On ......................... 21

Figure 3.6: Average Annual EOL Voltages for Prototypical Feeders with CVR Off ........................... 22

Figure 3.7: Average Annual EOL Voltages for Prototypical Feeders with CVR On............................ 22

Figure 3.8: Annual Energy Change $(\mathrm{kWh})$ by Taxonomy Feeder...................................................... 23

Figure 3.9: Annual Energy Change (\%) by Taxonomy Feeder .......................................................... 23

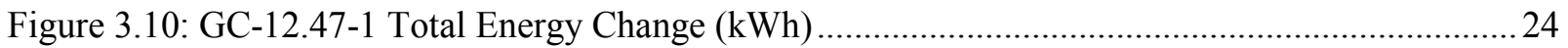

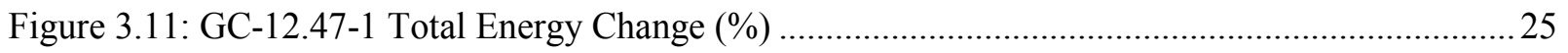

Figure 3.12: GC-12.47-1 Total Load Change (kWh) ...................................................................... 25

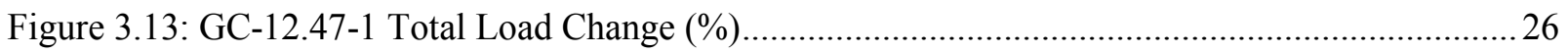

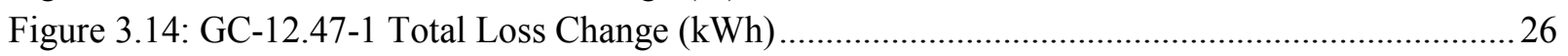

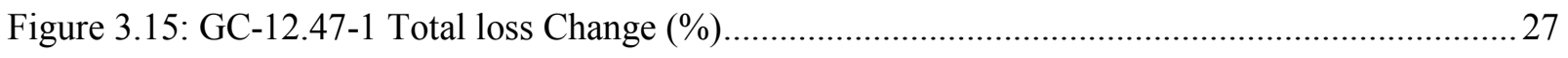

Figure 3.16: Comparison of Load and Loss Change (kWh) ......................................................... 27

Figure 4.1: Percent Total Benefit vs. Percent Total Number of Feeders in the United States ................ 31

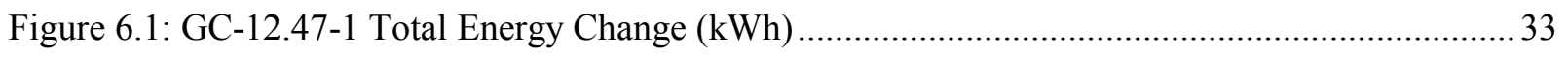

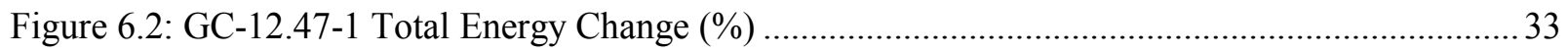

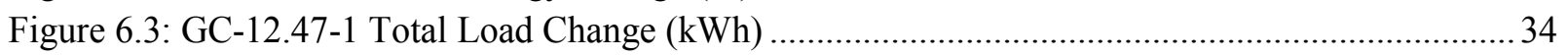

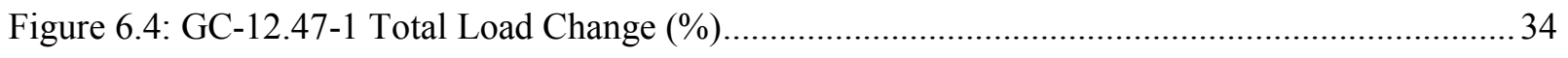

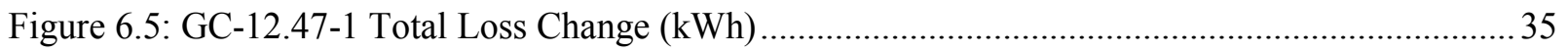

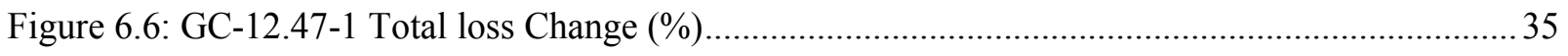

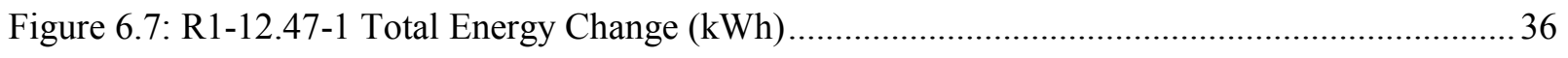

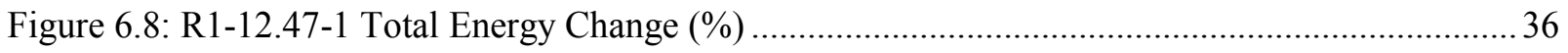

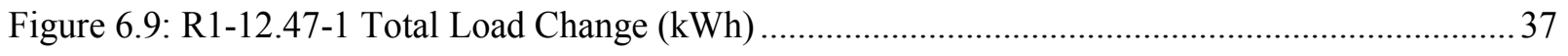

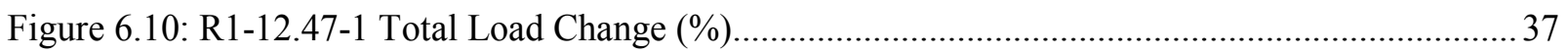

Figure 6.11: R1-12.47-1 Total Loss Change (kWh) ..................................................................... 38

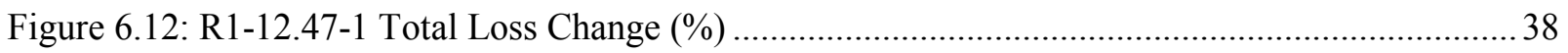

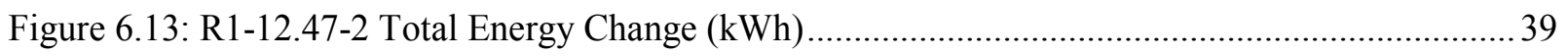

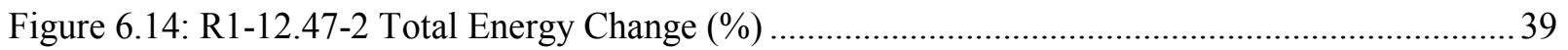




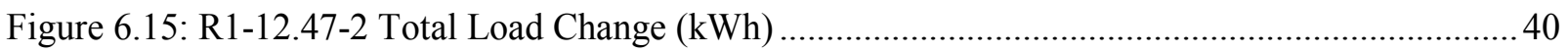

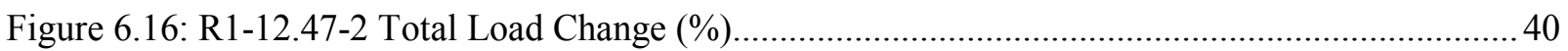

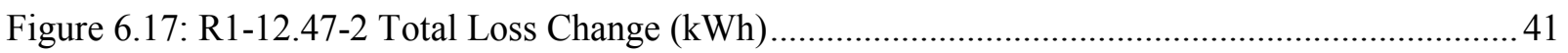

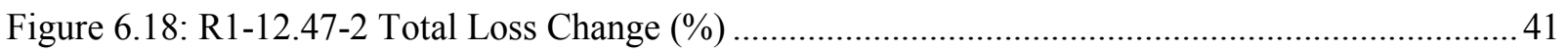

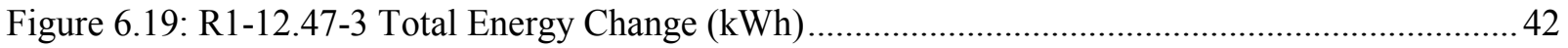

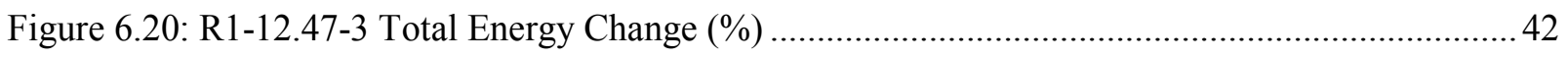

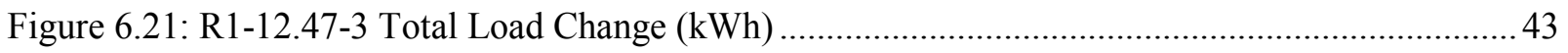

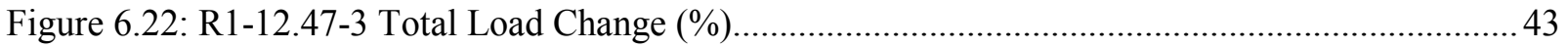

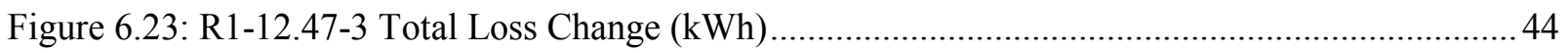

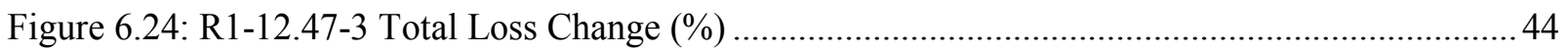

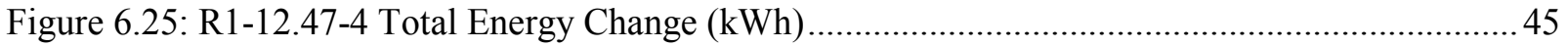

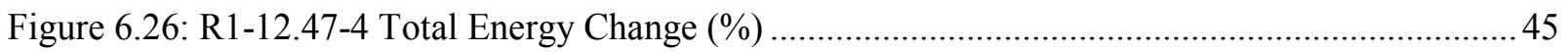

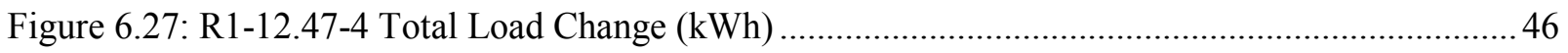

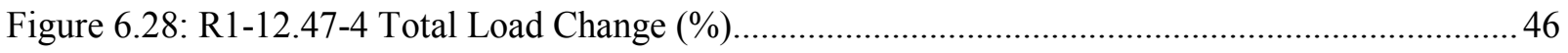

Figure 6.29: R1-12.47-4 Total Loss Change (kWh) ..................................................................... 47

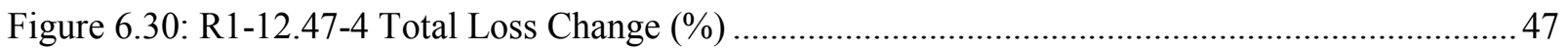

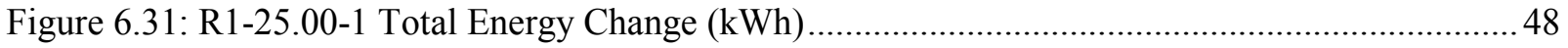

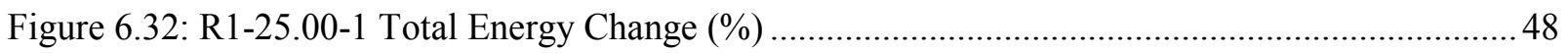

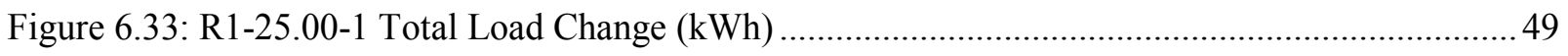

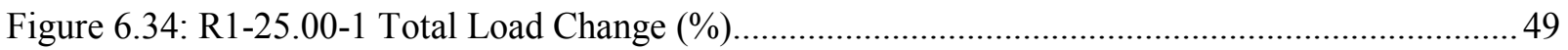

Figure 6.35: R1-25.00-1 Total Loss Change (kWh) .................................................................... 50

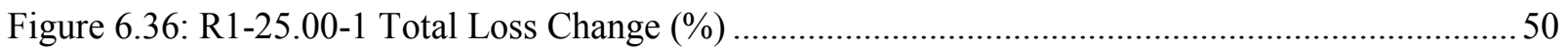

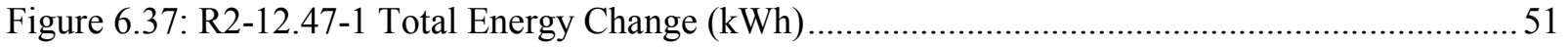

Figure 6.38: R2-12.47-1 Total Energy Change kWh ..................................................................... 51

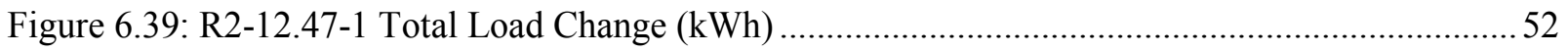

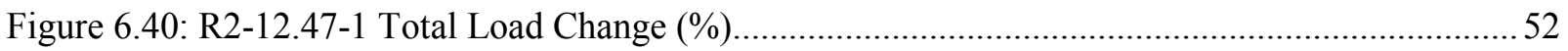

Figure 6.41: R2-12.47-1 Total Loss Change (kWh) ................................................................... 53

Figure 6.42: R2-12.47-1 Total Loss Change (\%) .......................................................................... 53

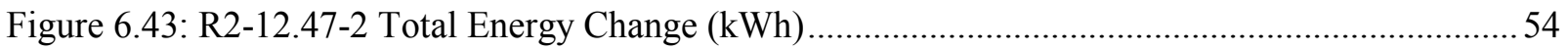

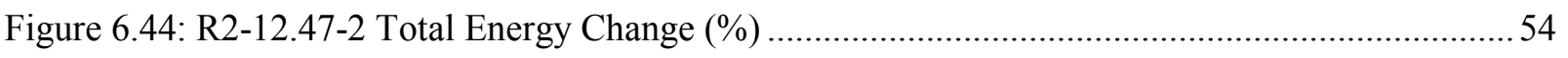

Figure 6.45: R2-12.47-2 Total Load Change $(\mathrm{kWh})$...................................................................... 55

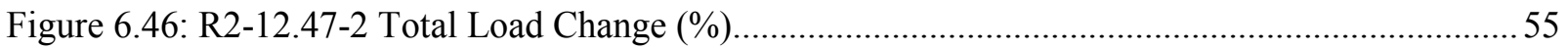

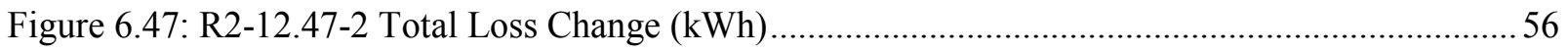

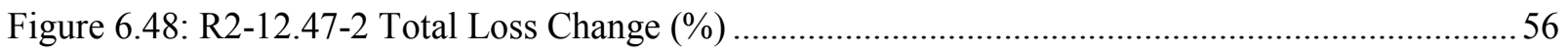

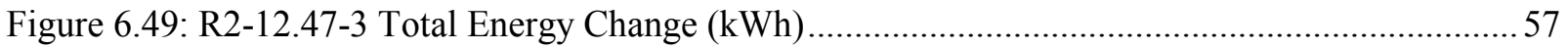

Figure 6.50: R2-12.47-3 Total Energy Change (\%) ....................................................................... 57

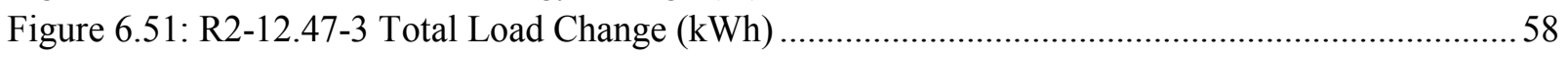

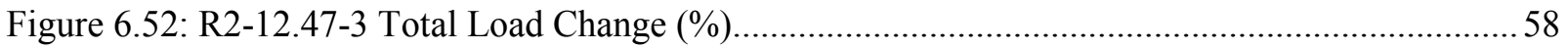

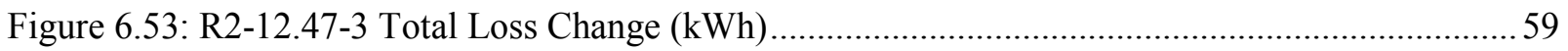

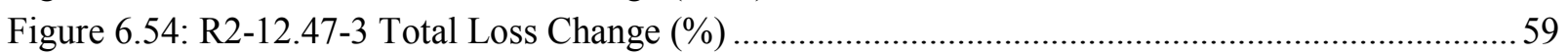

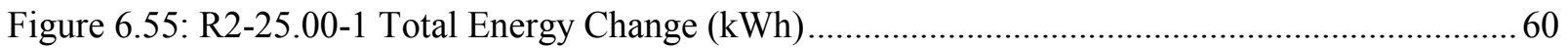

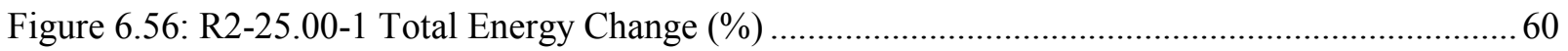

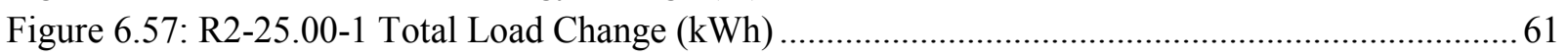

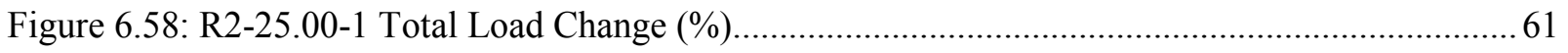




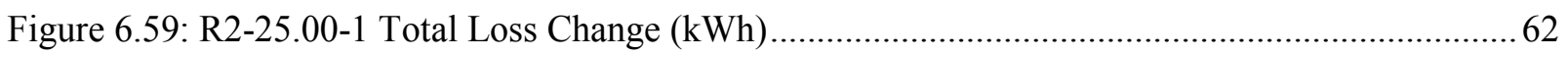

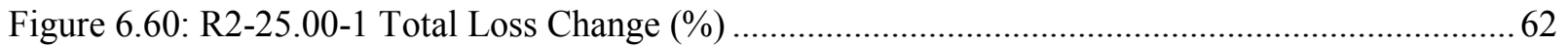

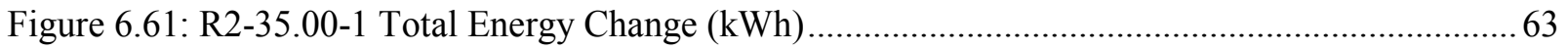

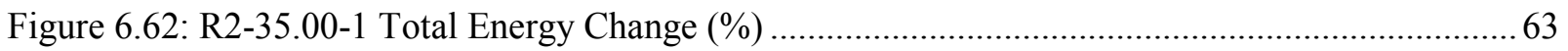

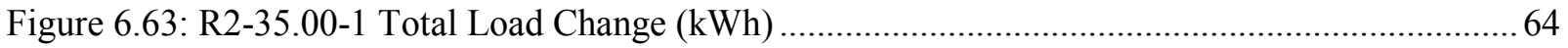

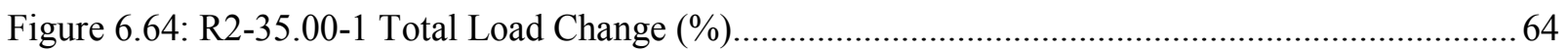

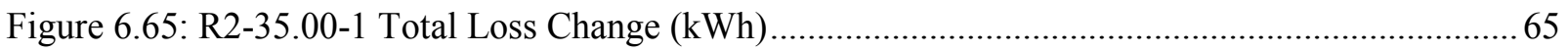

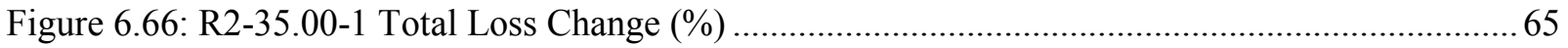

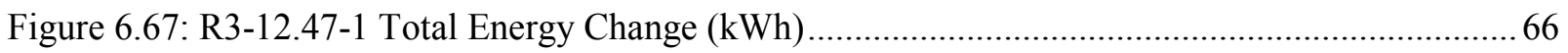

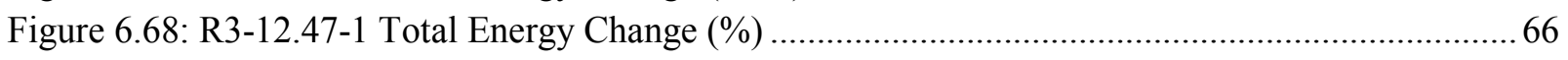

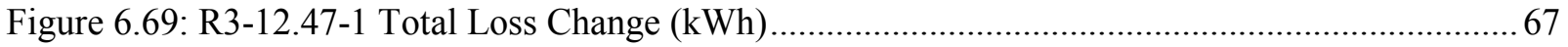

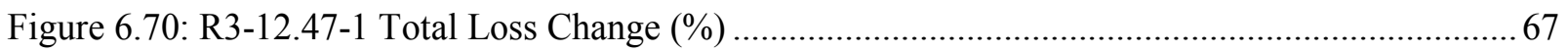

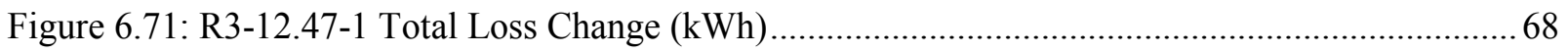

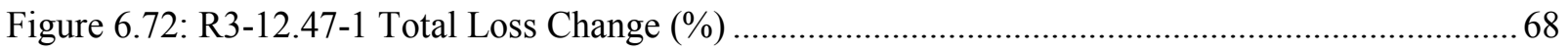

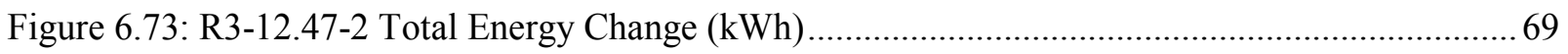

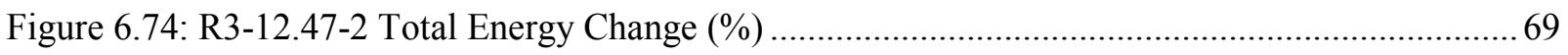

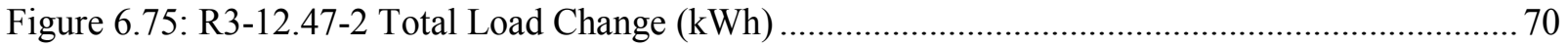

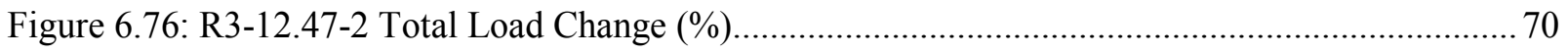

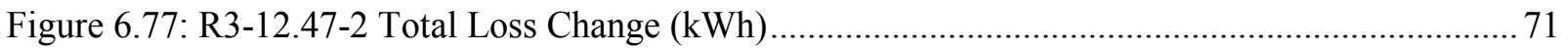

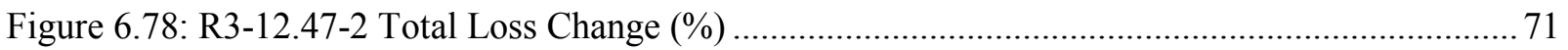

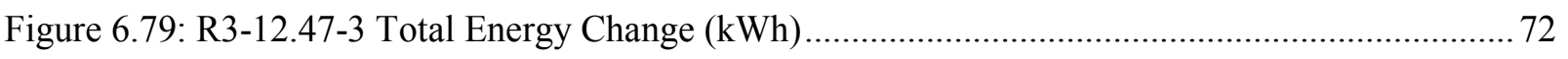

Figure 6.80: R3-12.47-3 Total Energy Change (\%) ...................................................................... 72

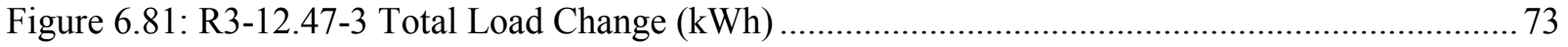

Figure 6.82: R3-12.47-3 Total Load Change (\%)...................................................................... 73

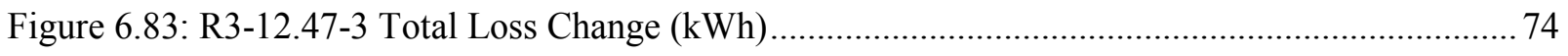

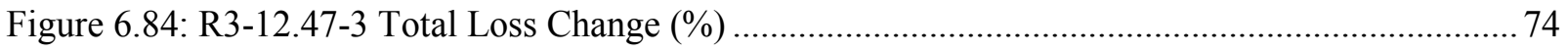

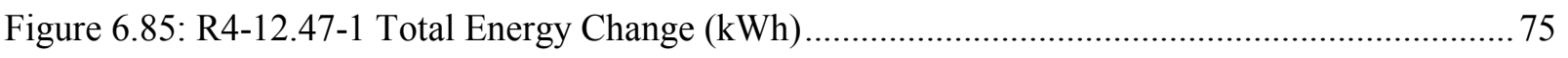

Figure 6.86: R4-12.47-1 Total Energy Change (\%) ...................................................................... 75

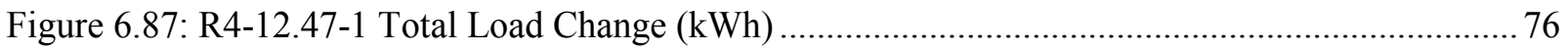

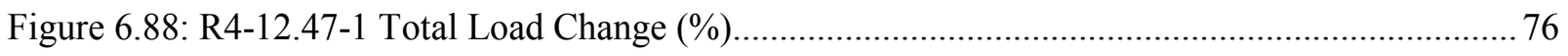

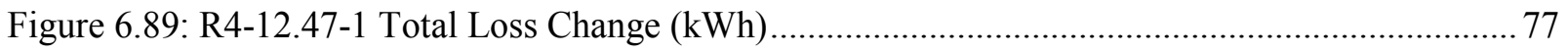

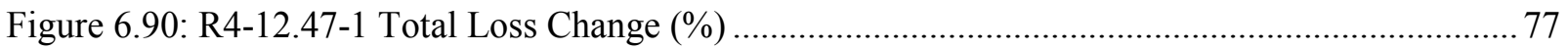

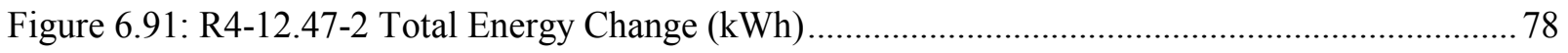

Figure 6.92: R4-12.47-2 Total Energy Change (\%) ........................................................................ 78

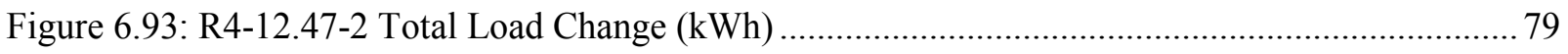

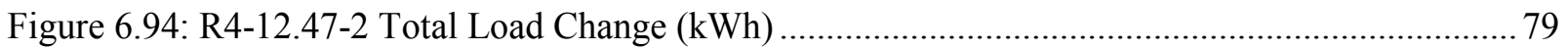

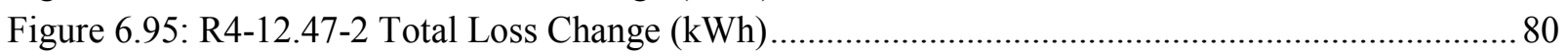

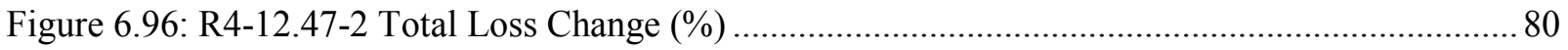

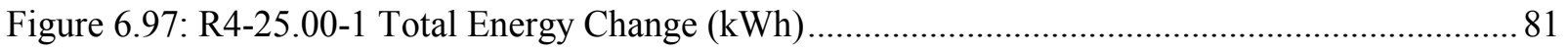

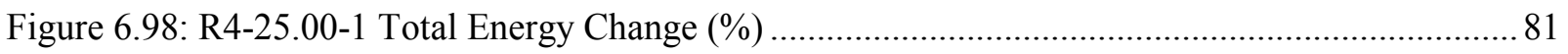

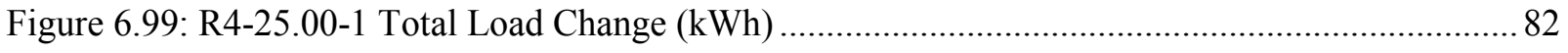

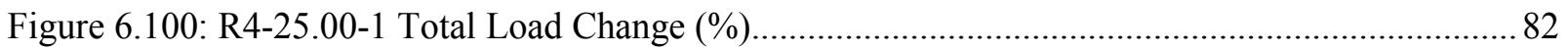

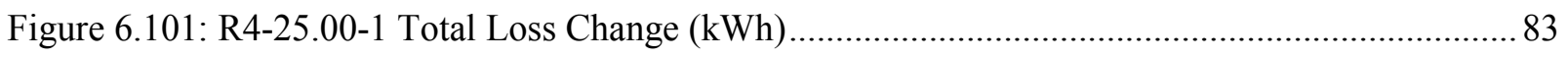

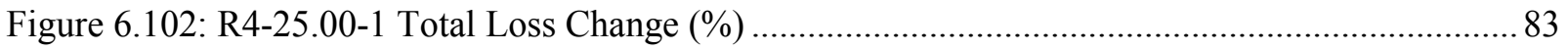




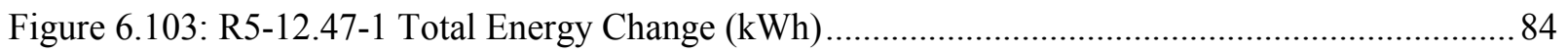

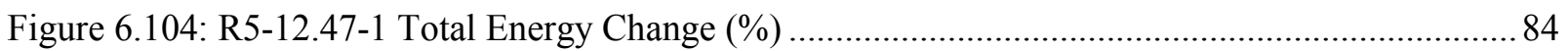

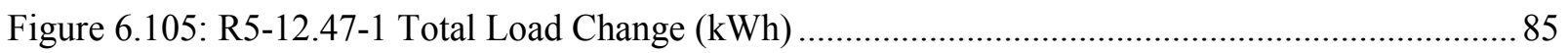

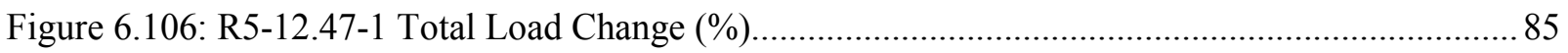

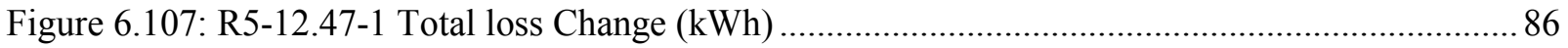

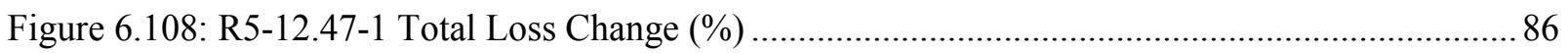

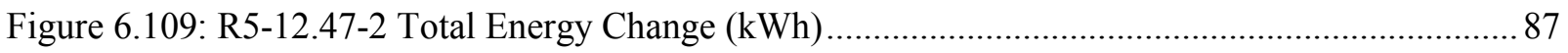

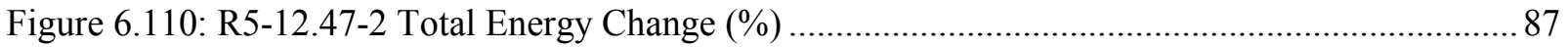

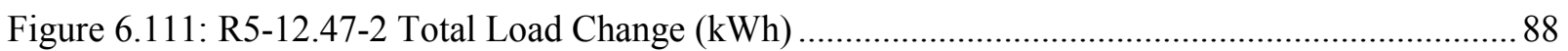

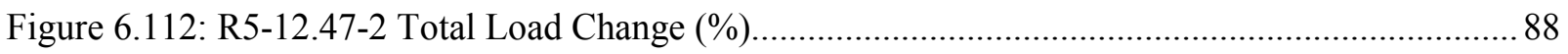

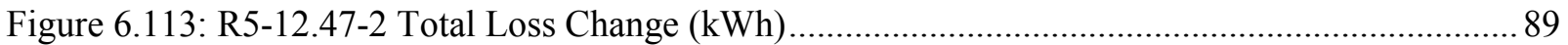

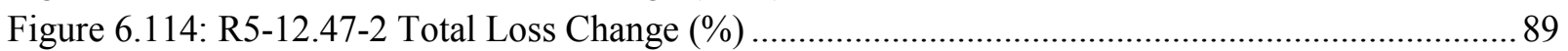

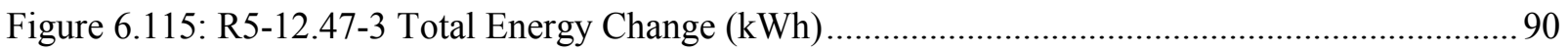

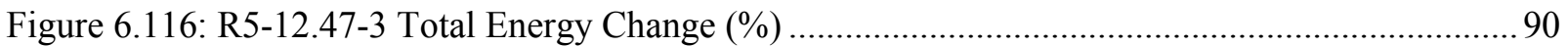

Figure 6.117: R5-12.47-3 Total Load Change (kWh) ...................................................................... 91

Figure 6.118: R5-12.47-3 Total Load Change (\%)......................................................................... 91

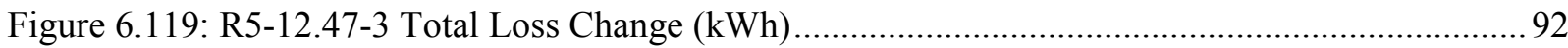

Figure 6.120: R5-12.47-3 Total Loss Change (\%) ........................................................................ 92

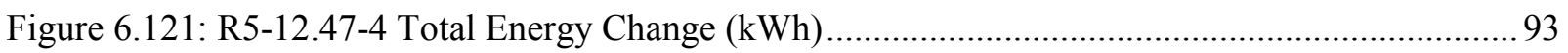

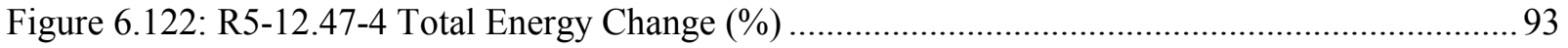

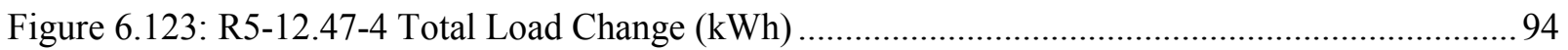

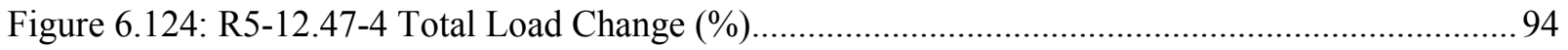

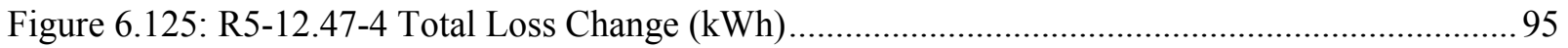

Figure 6.126: R5-12.47-4 Total Loss Change (\%) ........................................................................... 95

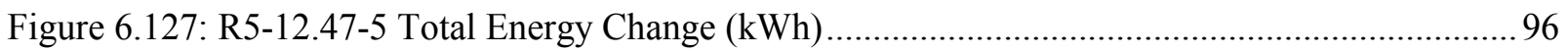

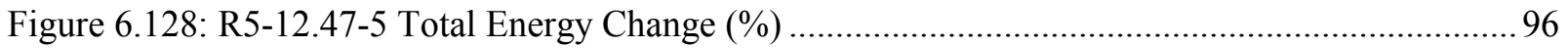

Figure 6.129: R5-12.47-5 Total Load Change (kWh) …................................................................. 97

Figure 6.130: R5-12.47-5 Total Load Change (\%)............................................................................ 97

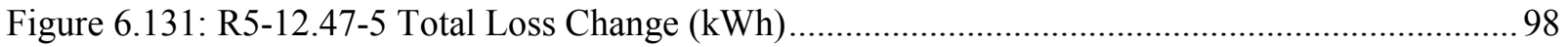

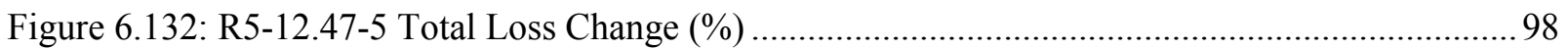

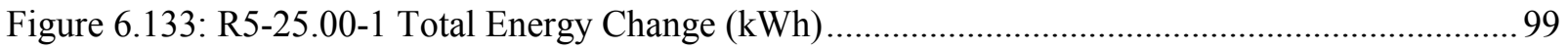

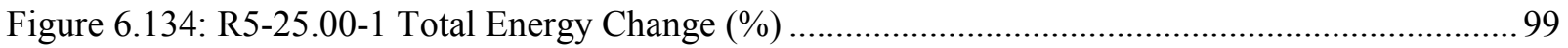

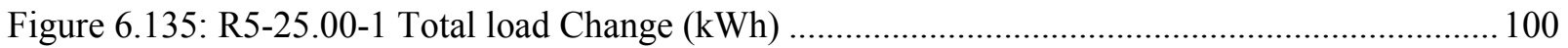

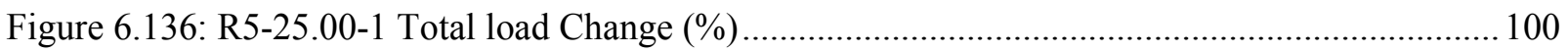

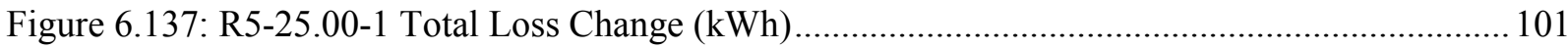

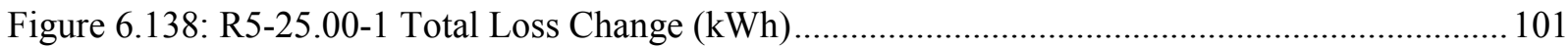

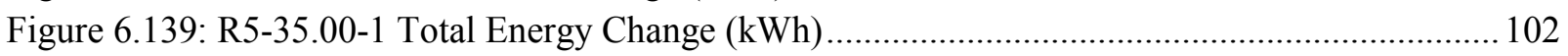

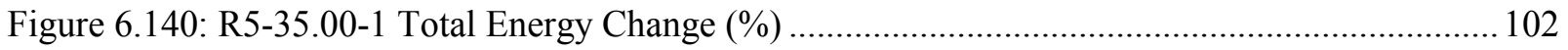

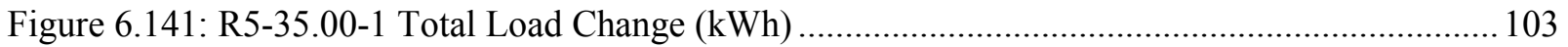

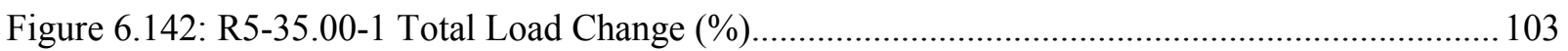

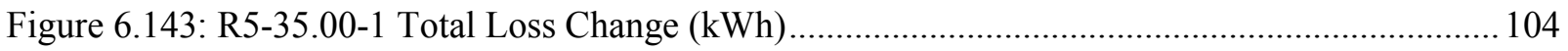

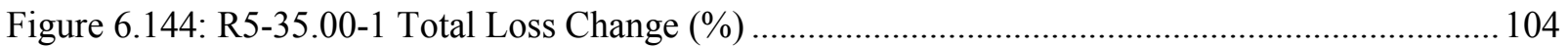




\section{Table of Tables}

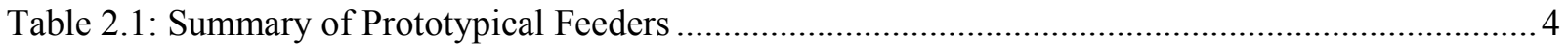

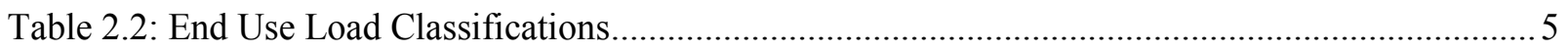

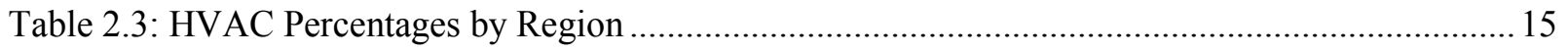

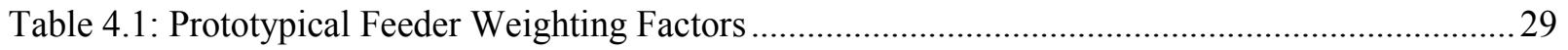

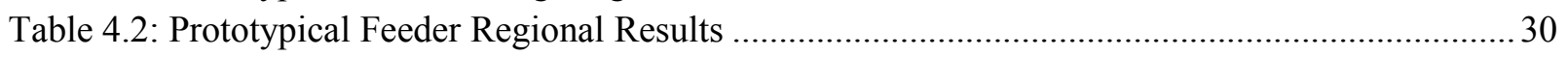




\section{Executive Summary}

This report has developed an estimate of the benefits of Conservation Voltage Reduction (CVR) for individual distribution feeder types, as well as an extrapolation of the benefits on a national level. Simulations were conducted using the GridLAB-D simulation environment, developed at the Pacific Northwest National Laboratory (PNNL), as well as the Taxonomy of Prototypical Feeders developed under the Modern Grid Initiative (MGI), now the Modern Grid Strategy (MGS). Based on the results of this report there are seven high level conclusions:

1) The analysis of CVR, as well as other smart grid technologies, requires the use of time-series simulations.

2) The behavior of end use loads is more complicated than generally acknowledged. Voltage dependent multi-state models must be used to accurately represent the effects of CVR.

3) CVR provides peak load reduction and annual energy reduction of approximately $0.5 \%-4 \%$ depending on the specific feeder.

4) When extrapolated to a national level, it can be seen that a complete deployment of CVR, $100 \%$ of distribution feeders, provides a $3.04 \%$ reduction in annual energy consumption.

5) If deployed only on high value distribution feeders, $40 \%$ of distribution feeders, the annual energy consumption is still reduced by $2.4 \%$.

6) In a practical deployment of CVR heavily loaded, higher voltage feeders should be targeted.

7) Loss reduction is not a significant benefit of CVR. 


\section{Introduction}

Conservation Voltage Reduction (CVR) is a reduction of energy consumption resulting from a reduction of feeder voltage. While there have been numerous CVR systems deployed in North America, there has been little substantive analytic analysis of the effect; the majority of the published results are based on empirical field measurements. As a result, it is difficult to extrapolate how this technology will behave on the various types of distribution feeders found throughout the nation.

To ensure that the results of this report can be reproduced by other researchers, all of the tools, models, and materials used are openly available at [http://sourceforge.net/projects/gridlab-d/]. In order to prevent showing bias to any particular commercial vendor, the method of CVR selected was from a twenty year old academic publication. While this method of CVR does not represent the current state of the art, it does contain the fundamental elements that are used in current commercial CVR schemes. The majority of CVR schemes contain two fundamental components: reactive power compensation and voltage optimization. Reactive power compensation is achieved through the operation of shunt capacitors in order to maintain the power factor at the substation transformer within a prescribed band. Voltage optimization is achieved through the operation of substation voltage regulators in order to regulate the voltage at specific End of Line (EOL) points within a prescribed range. In this way the peak load is reduced and the annual energy consumption is reduced.

Through detailed time-series simulations conducted in GridLAB-D, the effectiveness of CVR can be examined on each of the 24 Prototypical Feeders. The weighting factors developed in [1] are then used to extrapolate these results to a national level. This methodology allows for the operational impact of CVR to be analyzed from the device level to the national level.

The remainder of this report is divided into five additional sections. Section 2 discusses the level of complexity which must be included in simulations in order to effectively evaluate CVR, while Section 3 examines the simulation results of the 24 Prototypical Feeders. Section 4 extrapolates the individual feeder results of Section 3 in order to develop a national level estimate of the benefits of CVR and Section 5 contains the concluding remarks. Section 6 is an appendix which contains multiple analysis plots for each of the 24 Prototypical Feeders. 


\section{Modeling Principles}

In order to effectively model CVR, as well as most distribution level behaviors, it is necessary to perform time series simulations. Examining the peak load behavior and inferring behavior for the rest of the year is not adequate. For the analysis of CVR presented in this report simulations were performed with a one (1) minute time step for an entire year (8760 hours).

Additionally, standard power flow solutions are insufficient for analyzing the effects of CVR. Many loads within distribution systems cannot be defined as simple constant impedance, constant current, and constant power loads (ZIP). Many are thermostatically controlled, provide constant mechanical power, or draw a constant amount of energy over different time periods. To properly understand the effects of voltage reduction on the distribution system, such loads must be properly modeled. Additionally, standard distribution solvers ignore the effects of residential transformers (typically split-phase or center-tapped) and the cabling that connects the consumer to the transformer. While omitting these components may be acceptable for traditional capacity planning studies, when studying the effects of voltage reduction, they must be included. This section will discuss the level of detail which was used for determining the impacts of CVR on the various prototypical distribution feeders.

\subsection{Taxonomy of Prototypical Distribution Feeders}

As part of the 2008 MGI efforts, a Taxonomy of Prototypical Distribution Feeders was developed [1]. The feeders within this taxonomy were designed to provide researchers with an openly available set of distribution feeder models which are representative of those seen in the continental United States. Because climate and energy consumption are closely coupled, the prototypical feeders were divided into five climate regions based on the U.S DOE handbook (1980) providing design guidance for energy-efficient small office buildings [2].

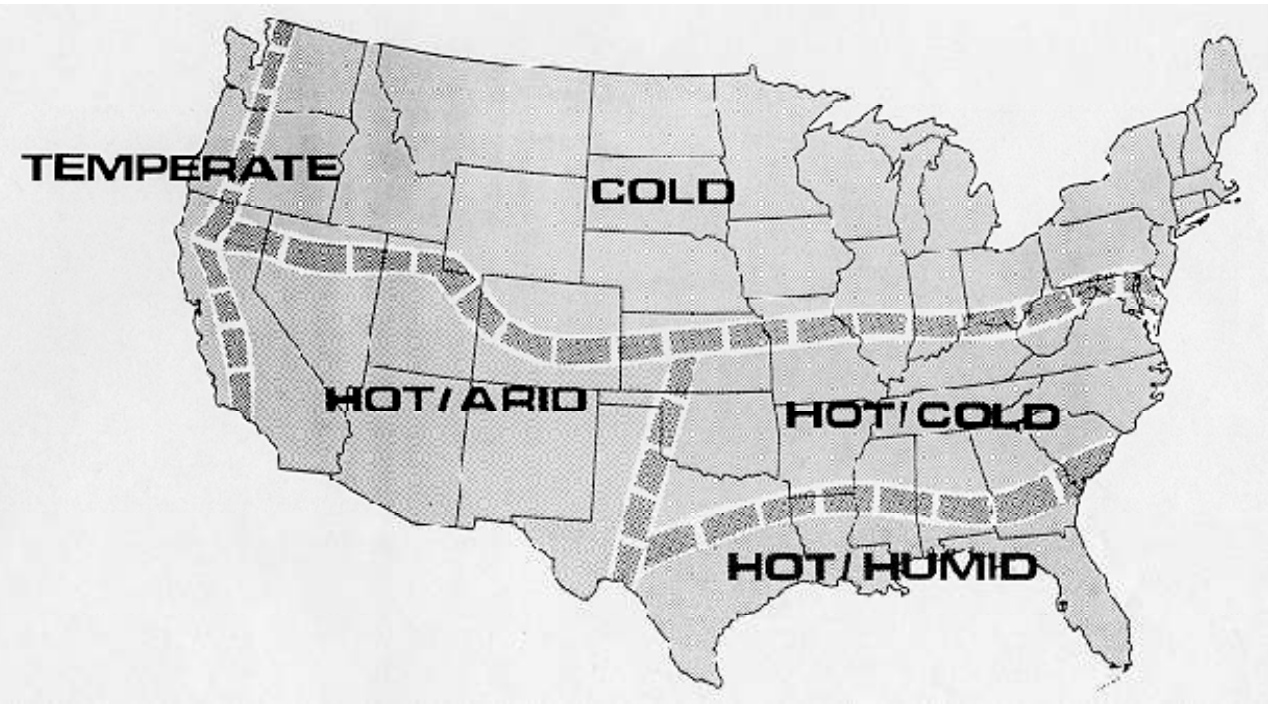

Figure 2.1: Climate Zones Used for Development of Prototypical Feeders 
Within each of the climate zones, there are a set of feeders that are approximations of the types of feeders that are seen within that zone. Table 2.1 gives a summary of the 24 prototypical feeders, including feeder name, base voltage, peak load, and a qualitative description. The peak loading used for the CVR analysis is slightly different than the original values from the 2008 report; this difference will be discussed in further sections.

Table 2.1: Summary of Prototypical Feeders

\begin{tabular}{|c|r|r|l|}
\hline Feeder & Base kV & Peak MVA & \multicolumn{1}{|c|}{ Description } \\
\hline R1-12.47-1 & 12.5 & 5.4 & Moderate suburban and rural \\
\hline R1-12.47-2 & 12.47 & 4.3 & Moderate suburban and light rural \\
\hline R1-12.47-3 & 12.47 & 2.4 & Small urban center \\
\hline R1-12.47-4 & 12.47 & 1.8 & Heavy suburban \\
\hline R1-25.00-1 & 24.9 & 4.9 & Light rural \\
\hline R2-12.47-1 & 12.47 & 2.3 & Light urban \\
\hline R2-12.47-2 & 12.47 & 6.7 & Moderate suburban \\
\hline R2-12.47-3 & 12.47 & 6.7 & Light suburban \\
\hline R2-25.00-1 & 24.9 & 4.8 & Moderate urban \\
\hline R2-35.00-1 & 34.5 & 21.3 & Light rural \\
\hline R3-12.47-1 & 12.47 & 6.9 & Heavy urban \\
\hline R3-12.47-2 & 12.47 & 11.6 & Moderate urban \\
\hline R3-12.47-3 & 12.47 & 4.0 & Heavy suburban \\
\hline R4-12.47-1 & 13.8 & 9.4 & Heavy urban with rural spur \\
\hline R4-12.47-2 & 12.5 & 6.7 & Light suburban and moderate urban \\
\hline R4-25.00-1 & 24.9 & 2.1 & Light rural \\
\hline R5-12.47-1 & 13.8 & 1.0 & Heavy suburban and moderate urban \\
\hline R5-12.47-2 & 12.47 & 10.8 & Moderate suburban and heavy urban \\
\hline R5-12.47-3 & 13.8 & 4.2 & Moderate rural \\
\hline R5-12.47-4 & 12.47 & 4.8 & Moderate suburban and urban \\
\hline R5-12.47-5 & 12.47 & 6.2 & Moderate suburban and light urban \\
\hline R5-25.00-1 & 22.9 & 8.5 & Heavy suburban and moderate urban \\
\hline R5-35.00-1 & 34.5 & 9.3 & Moderate suburban and light urban \\
\hline GC-12.47-1 & 12.47 & 12.1 & Single large commercial or industrial \\
\hline
\end{tabular}

The original prototypical feeders were modeled in detail from the substation to the end use point of interconnection, but did not include detailed load models. To use these feeders for an accurate analytic assessment of CVR, it was necessary to include detailed end use load models. 


\subsection{Determination of Load Type}

Load information in the original feeder models was fairly limited. The original models contained a small amount of information on commercial loads and no information on residential loads. Loads were defined as static spot loads, where blocks of individual commercial and residential loads were summed to a single peak spot load on the primary system (no secondary voltage loads were defined). To more accurately classify the loads, Google Earth $\subseteq$ images of the feeders were located and the physical dimensions of the feeder overlaid. The loads provided by the original model were then manually classified by the type of building found at that location, and were broken into nine different load types via visual inspection. These were classified as Residential 1-6, Commercial 1-2, and Industrial. Brief descriptions are provided in Table 2.2. Each load classification describes the properties of the load in that area, and the details that describe each type of load will be further described in Section 2.4 Population of Loads.

Table 2.2: End Use Load Classifications

\begin{tabular}{|l|l|}
\hline \multicolumn{1}{|c|}{ Load Class } & \multicolumn{1}{c|}{ Description } \\
\hline Residential 1 & Pre-1980 $<2000$ sqft. \\
\hline Residential 2 & Post-1980 $<2000$ sqft. \\
\hline Residential 3 & Pre-1980 $>2000$ sqft. \\
\hline Residential 4 & Post-1980 $>2000$ sqft. \\
\hline Residential 5 & Mobile Homes \\
\hline Residential 6 & Apartment Complex \\
\hline Commercial 1 & $>35 \mathrm{kVA}$ \\
\hline Commercial 2 & $<35 \mathrm{kVA}$ \\
\hline Industrial & All Industrial \\
\hline
\end{tabular}

By defining each building as older or newer, and larger or smaller, approximate physical properties for those homes could be assumed. These were then used to define multiple building models at each load location, depending upon the type of building that was found through observation in Google Earth $\subset$. Defining these properties gives insight into the benefits of voltage reduction not only at a single given load level, but as a function of seasonal and daily variations in load. Once again, while a particular building model at that location does not accurately represent a specific building in reality, the aggregate of the distribution of the buildings should approximate the response of all of the real buildings. Within each building, appliance loads were also modeled, as will be seen in the following sections.

\subsection{Load Models}

Once each of the points of interconnection were classified in accordance with Table 2.2, it was necessary to fully represent the load. Because of the complexity of end use load behavior, load 
models can be divided into two distinct classes: those without thermal cycles and those with thermal cycles. Loads without thermal cycles consume energy in a time-invariant manner, with the exception of voltage variations. Specifically, there is no control feedback loop. As an example, a light bulb will consume energy when turned on, as a function of voltage, in a fixed manner. In contrast, a load with a thermal cycle, such as a hot water heater, will have a varying duty cycle dependent on the supply voltage. For example, if the supply voltage is lowered, the hot water heater will draw less instantaneous power, but it must remain on for a longer period in order to heat the same mass of water.

Sections 2.3.1 and 2.3.2 will discuss loads without thermal cycles while Section 2.3.3 will discuss loads with thermal cycles. Section 2.3.4 will then discuss how these were combined to form complete load models for individual Residential, Commercial, and Industrial Customers.

\subsubsection{Loads without Thermal Cycles}

The traditional method for modeling a load without a thermal cycle is to use a ZIP model. The ZIP model is a load which is composed of time-invariant constant impedance $(Z)$, constant current (I), and constant power (P) elements. Figure 2.2 shows the circuit representation of the ZIP model.

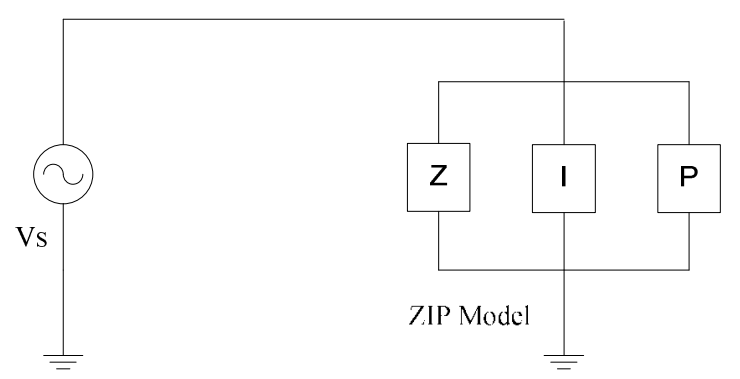

Figure 2.2: The Traditional ZIP Load Model

The total real power consumed by a ZIP load at a given voltage is shown in (2.1), and (2.2) gives the reactive power consumption. The values of the constants within (2.1) and (2.2) are limited by the constraint of (2.3)

$$
\begin{aligned}
& P_{i}=\frac{V_{a}^{2}}{V_{n}^{2}} \cdot S_{n} \cdot Z_{\%} \cdot \cos \left(Z_{\theta}\right)+\frac{V_{a}}{V_{n}} \cdot S_{n} \cdot I_{\%} \cdot \cos \left(I_{\theta}\right)+S_{n} \cdot P_{\%} \cdot \cos \left(P_{\theta}\right) \\
& Q_{i}=\frac{V_{a}^{2}}{V_{n}^{2}} \cdot S_{n} \cdot Z_{\%} \cdot \sin \left(Z_{\theta}\right)+\frac{V_{a}}{V_{n}} \cdot S_{n} \cdot I_{\%} \cdot \sin \left(I_{\theta}\right)+S_{n} \cdot P_{\%} \cdot \sin \left(P_{\theta}\right) \\
& 1=Z_{\%}+I_{\%}+P_{\%}
\end{aligned}
$$


where:

$P_{i}$ : Real power consumption of the $i^{\text {th }}$ load

$Q_{i}$ : Reactive power consumption of the $i^{\text {th }}$ load

$V_{a}$ : Actual terminal voltage

$V_{n}$ : Nominal terminal voltage

$S_{n}$ : Apparent power consumption at nominal voltage

$Z_{\%}$ : Fraction of load that is constant impedance

$I_{\%}$ : Fraction of load that is constant current

$P_{0}$ : Fraction of load that is constant power

$Z_{\theta}$ : Phase angle of the constant impedance component

$I_{\theta}$ : Phase angle of the constant current component

$P_{\theta}$ : Phase angle of the constant power component

In (2.1) and (2.2), there are six (6) constants that define the voltage dependent behavior of the ZIP load: $Z_{\%}, I_{\%}, P_{\%}, Z_{\theta}, I_{\theta}$, and $P_{\theta}$. Because CVR changes the voltage of a feeder, it is critical to understand how typical end use loads will respond to changes in voltage. Specifically, what are the six constants that accurately reflect various end use loads? For loads such as a heating element, it is clear that the load is $100 \% \mathrm{Z}$, but for more complicated loads such as a Liquid Crystal Display (LCD) or Compact Florescent Light (CFL), the proper ratios are not as apparent. In an attempt to determine accurate ZIP models, a number of common household end use appliances were operated over a voltage range from $100 \mathrm{~V}$ to $126 \mathrm{~V}$ and their power consumption recorded. A constrained least squares fit was then used to determine the proper ZIP values, for both real power and power factor, that give the proper voltage dependency for the loads.

The following subsections contain plots of the real and reactive power consumption, $P_{m}$ and $Q_{m}$ respectively, for various end use loads while operated between $100 \mathrm{~V}$ and $126 \mathrm{~V}$. The plots will also contain a red line indicating the voltage response curve using the fitted ZIP values, $P_{e}$ and $Q_{e}$. In addition to the plots, the values for the six (6) fundamental ZIP values will be given for each load. These values are the numbers that will be used to model these loads in the prototypical feeders. While the following subsections do not contain a comprehensive list of loads, they provide a representative sample of the types of loads that are found in residences. 


\subsubsection{Incandescent Light Bulb (70W)}
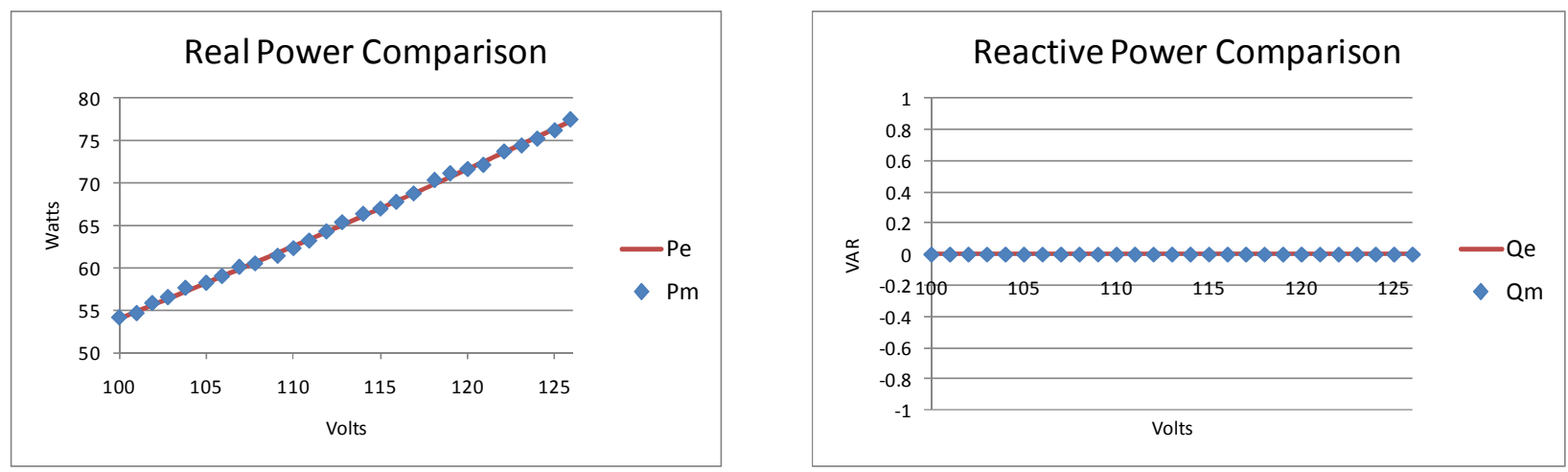

Figure 2.3: Voltage Dependent Energy Consumption of a 70W Incandescent Light Bulb

\begin{tabular}{|c|c|c|c|c|c|c|}
\cline { 2 - 7 } \multicolumn{1}{c|}{} & \multicolumn{6}{c|}{ ZIP Values } \\
\cline { 2 - 7 } \multicolumn{1}{c|}{} & Z-\% $\%$ & I-\% $\%$ & P-\% $\%$ & Z-pf & I- pf & P-pf \\
\hline Incandescent $75 \mathrm{~W}$ & $57.11 \%$ & $42.57 \%$ & $0.32 \%$ & 1.00 & -1.00 & 1.00 \\
\hline
\end{tabular}

\subsubsection{Magnavox Television (Cathode Ray Tube)}
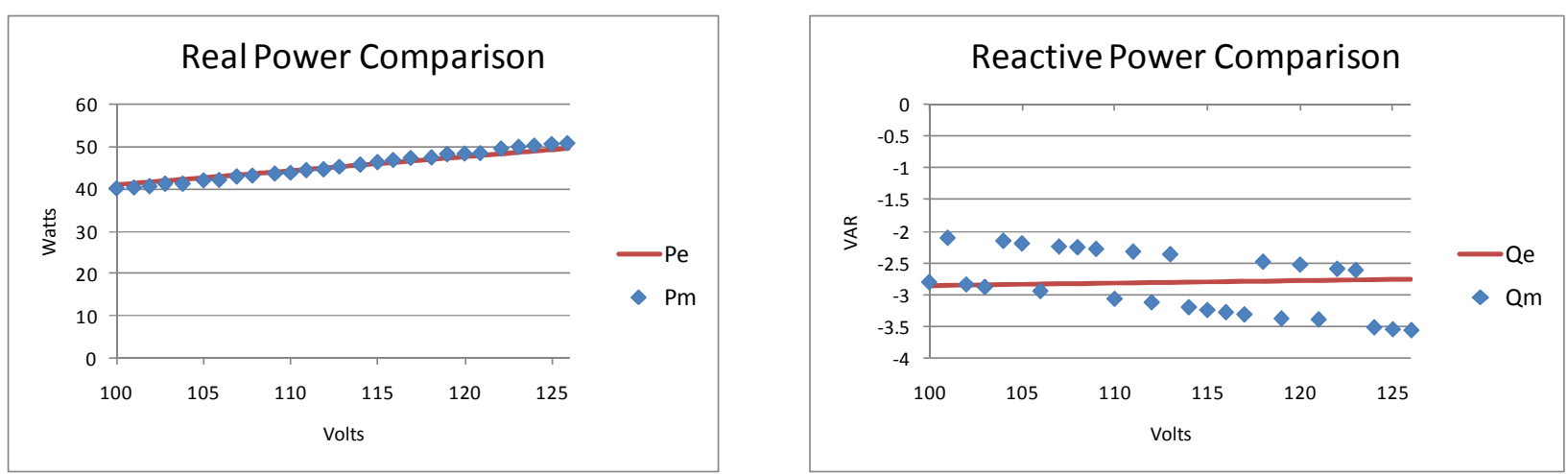

Figure 2.4: Voltage Dependent Energy Consumption of a CRT Television

\begin{tabular}{|c|c|c|c|c|c|c|}
\cline { 2 - 7 } \multicolumn{1}{c|}{} & \multicolumn{6}{c|}{ ZIP Values } \\
\cline { 2 - 7 } \multicolumn{1}{c|}{} & Z-\% & I-\% & P-\% & Z-pf & I-pf & P-pf \\
\hline TV-Magnavox CRT & $0.15 \%$ & $82.66 \%$ & $17.19 \%$ & -0.99 & 1.00 & -0.92 \\
\hline
\end{tabular}




\subsubsection{Oscillating Fan}
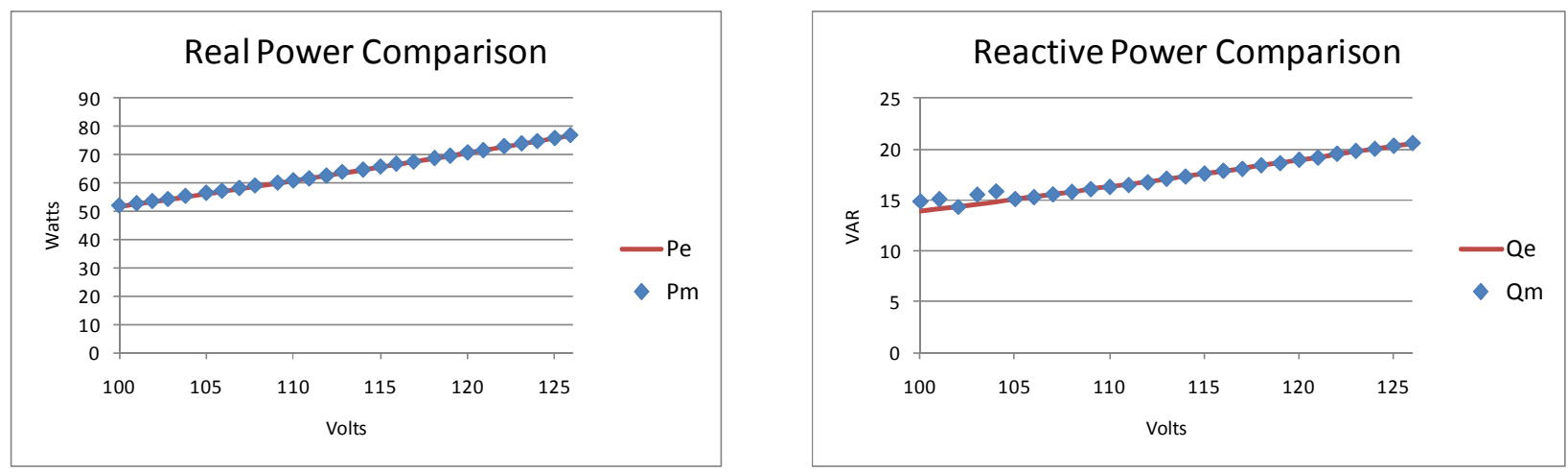

Figure 2.5: Voltage Dependent Energy Consumption of an Oscillating Fan

\begin{tabular}{|c|c|c|c|c|c|c|}
\cline { 2 - 7 } \multicolumn{1}{c|}{} & \multicolumn{6}{c|}{ ZIP Values } \\
\cline { 2 - 7 } \multicolumn{1}{c|}{ Z-\% $\%$} & I-\% & P-\% & Z-pf & I-pf & P-pf \\
\hline Oscillating Fan & $73.32 \%$ & $25.34 \%$ & $1.35 \%$ & 0.97 & 0.95 & -1.00 \\
\hline
\end{tabular}

\subsubsection{Liquid Crystal Display (LCD) - Dell}
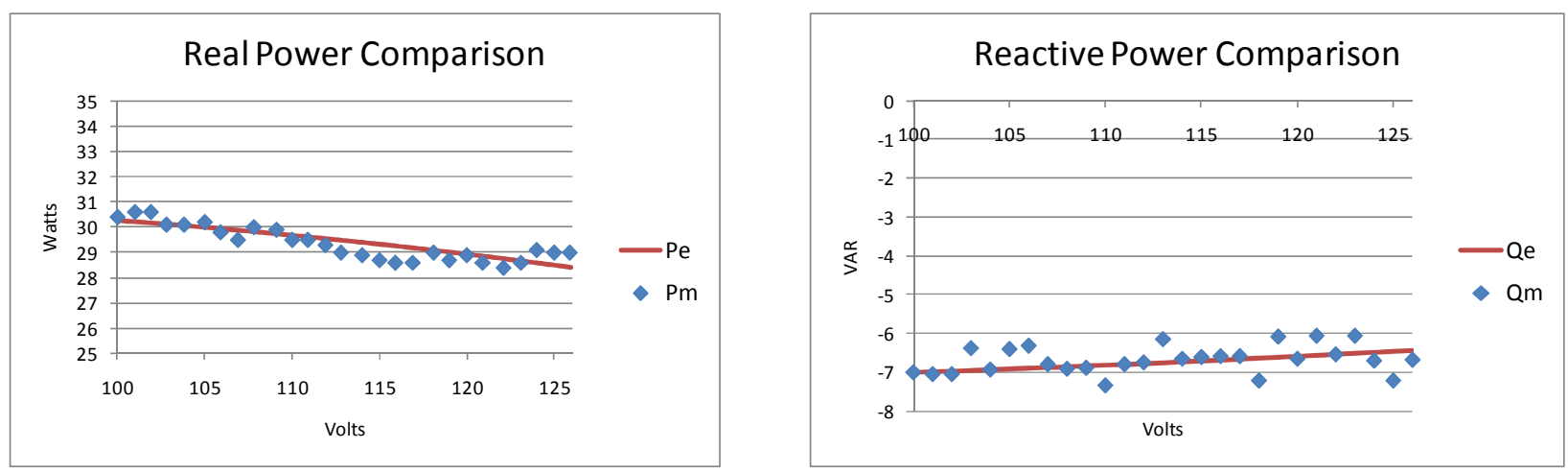

Figure 2.6: Voltage Dependent Energy Consumption of a Dell LCD

\begin{tabular}{|c|c|c|c|c|c|c|}
\cline { 2 - 7 } \multicolumn{1}{c|}{} & \multicolumn{6}{|c|}{ ZIP Values } \\
\cline { 2 - 7 } & Z-\% $\%$ & I-\% & P-\% & Z-pf & I- pf & P-pf \\
\hline LCD - Dell & $-40.70 \%$ & $46.29 \%$ & $94.41 \%$ & -0.97 & -0.98 & -0.97 \\
\hline
\end{tabular}


2.3.1.5. Plasma TV - Sony

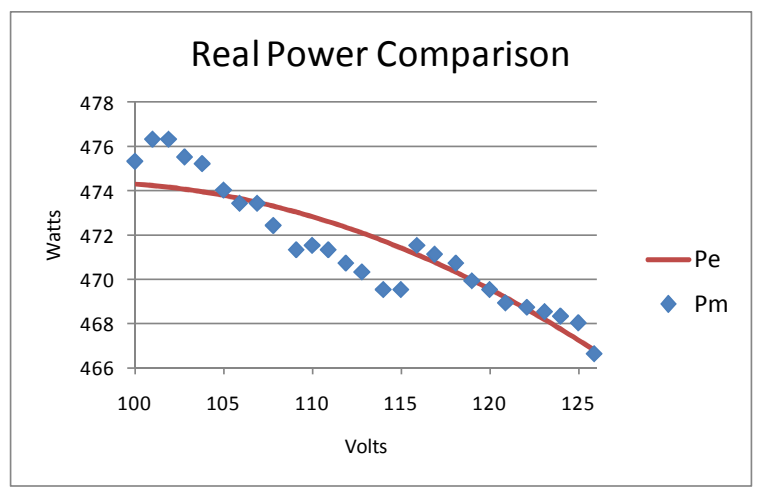

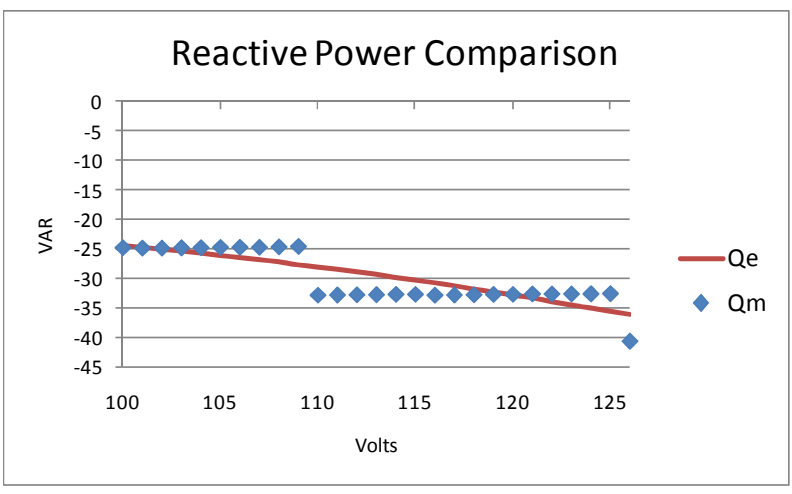

Figure 2.7: Voltage Dependent Energy Consumption of a Sony Plasma

\begin{tabular}{|c|c|c|c|c|c|c|}
\cline { 2 - 7 } \multicolumn{1}{c|}{} & \multicolumn{6}{c|}{ ZIP Values } \\
\cline { 2 - 8 } & Z-\% & I-\% & P-\% & Z-pf & I- pf & P-pf \\
\hline Plasma - Sony & $-32.07 \%$ & $48.36 \%$ & $83.71 \%$ & 0.85 & 0.91 & -0.99 \\
\hline
\end{tabular}

\subsubsection{Liquid Crystal Display (LCD) - Clarity TV}
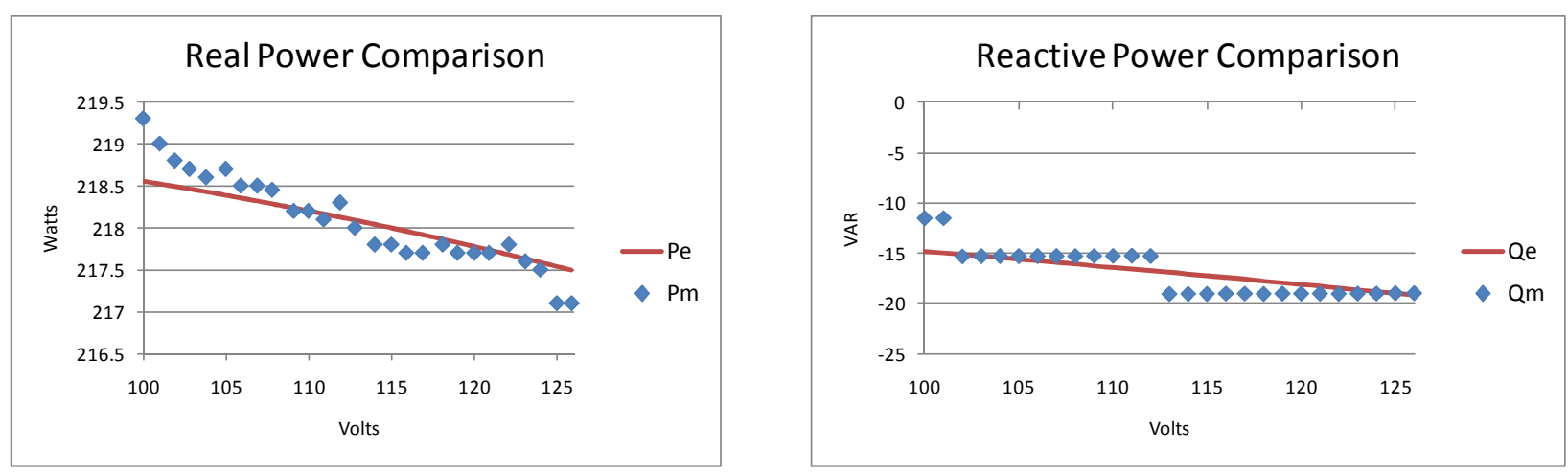

Figure 2.8: Voltage Dependent Energy Consumption of a Clarity LCD

\begin{tabular}{|l|l|l|c|c|c|c|}
\cline { 2 - 7 } \multicolumn{1}{c|}{} & \multicolumn{6}{c|}{ ZIP Values } \\
\cline { 2 - 7 } \multicolumn{1}{c|}{} & Z-\% $\%$ & I-\% & P-\% $\%$ & Z-pf & I- pf & P-pf \\
\hline LCD - Clarity & $-3.83 \%$ & $3.96 \%$ & $99.87 \%$ & 0.61 & -0.54 & -1.00 \\
\hline
\end{tabular}




\subsubsection{Compact Fluorescent Light (CFL) 13W}
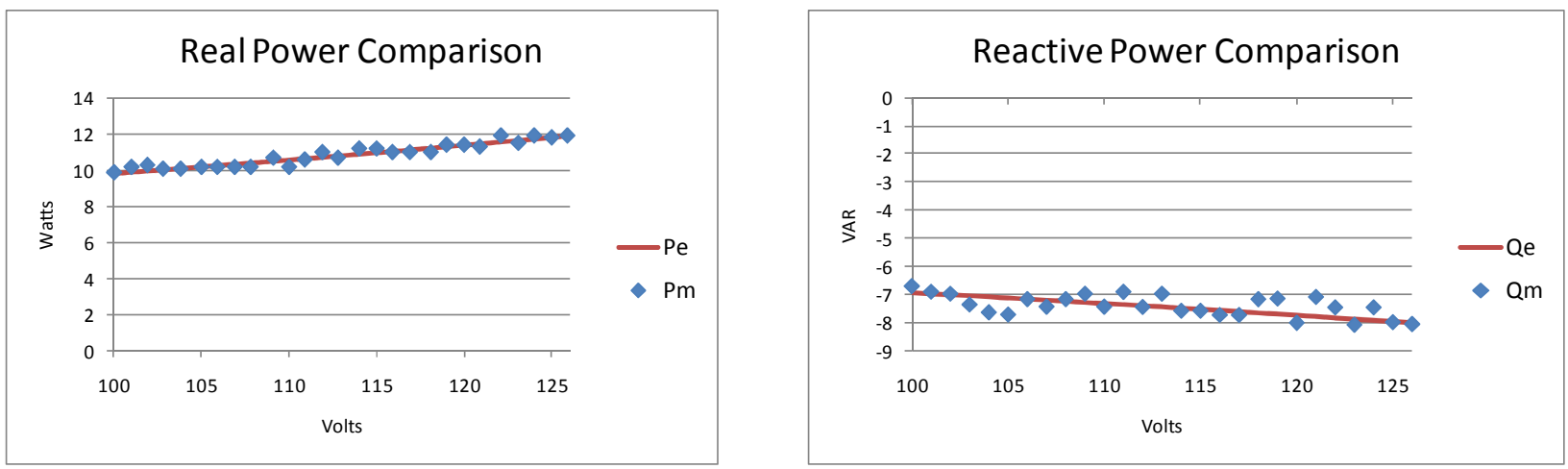

Figure 2.9: Voltage Dependent Energy Consumption of a 13W CFL

\begin{tabular}{|l|l|l|c|c|c|c|}
\cline { 2 - 7 } \multicolumn{1}{c|}{} & \multicolumn{6}{c|}{ ZIP Values } \\
\cline { 2 - 7 } \multicolumn{1}{c|}{} & Z-\% $\%$ & I-\% & P-\% $\%$ & Z-pf & I- pf & P-pf \\
\hline CFL-13W & $40.85 \%$ & $0.67 \%$ & $58.49 \%$ & -0.88 & 0.42 & -0.78 \\
\hline
\end{tabular}

\subsubsection{Compact Fluorescent Light (CFL) 20W}
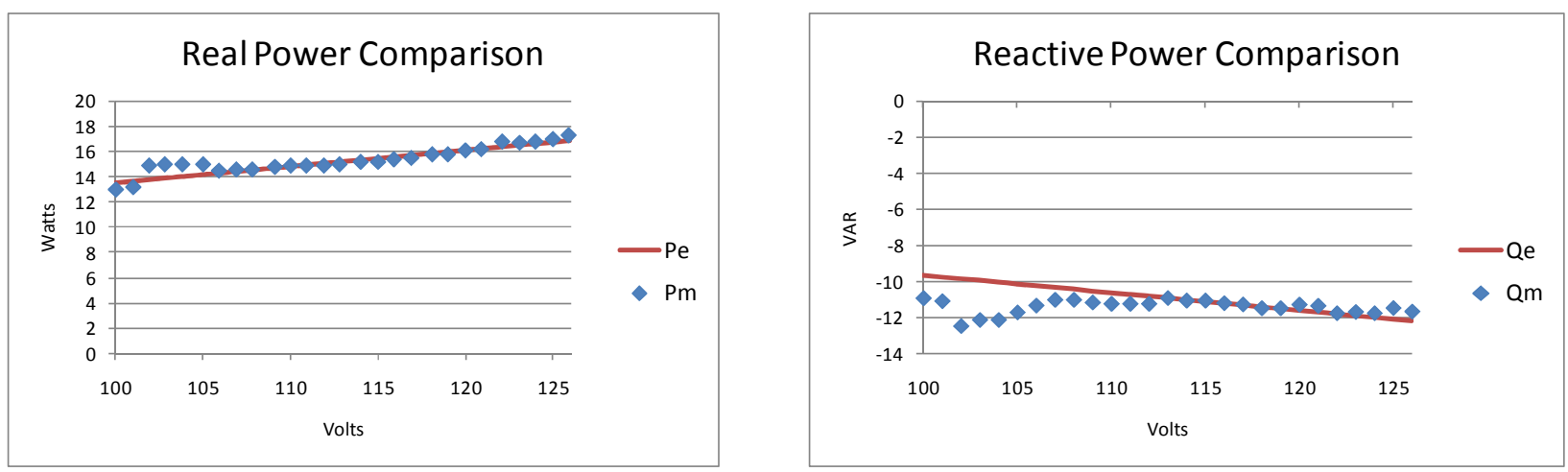

Figure 2.10: Voltage Dependent Energy Consumption of a 20W CFL

\begin{tabular}{|l|l|l|c|c|c|c|}
\cline { 2 - 7 } \multicolumn{1}{c|}{} & \multicolumn{6}{c|}{ ZIP Values } \\
\cline { 2 - 7 } \multicolumn{1}{c|}{} & Z-\% $\%$ & I-\% & P-\% & Z-pf & I- pf & P-pf \\
\hline CFL-20W & $-1.05 \%$ & $100.00 \%$ & $1.05 \%$ & 0.00 & -0.81 & 0.90 \\
\hline
\end{tabular}




\subsubsection{Compact Fluorescent Light (CFL) 42W}
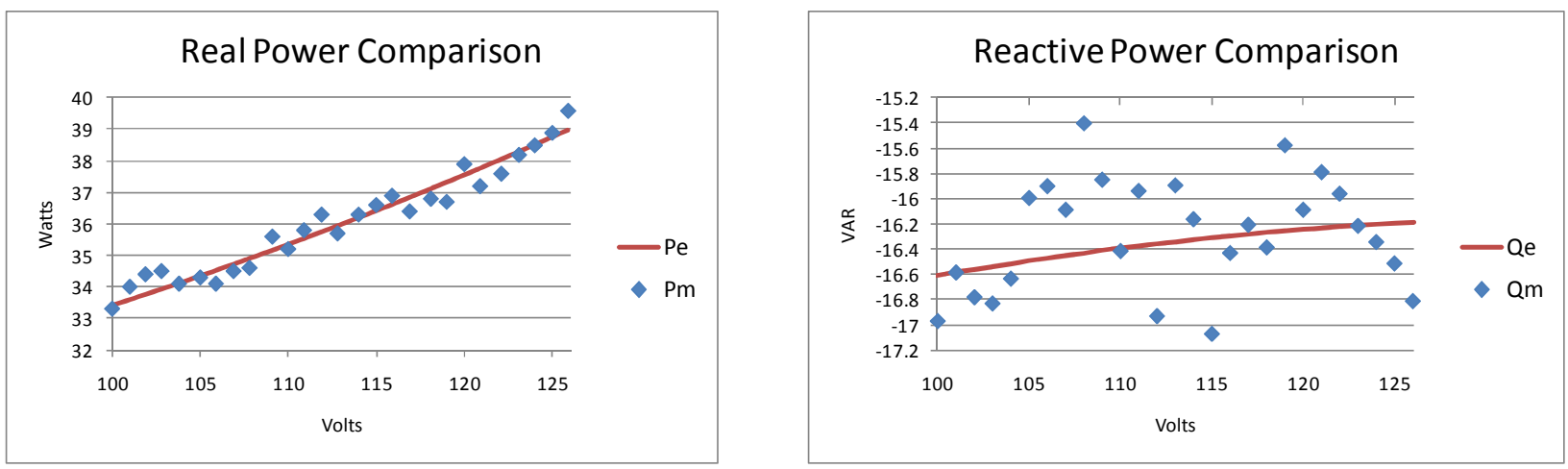

Figure 2.11: Voltage Dependent Energy Consumption of a 42W CFL

\begin{tabular}{|l|l|l|c|c|c|c|}
\cline { 2 - 7 } \multicolumn{1}{c|}{} & \multicolumn{6}{c|}{ ZIP Values } \\
\cline { 2 - 7 } \multicolumn{1}{c|}{} & Z-\% $\%$ & I-\% $\%$ & P-\% & Z-pf & I- pf & P-pf \\
\hline CFL-42W & $48.67 \%$ & $-37.52 \%$ & $88.84 \%$ & -0.97 & -0.70 & -0.79 \\
\hline
\end{tabular}

\subsubsection{Observations of ZIP Values}

As can be seen in the plots, and ZIP values obtained through the least squares fit from Sections 2.3.1.1 through 2.3.1.9, the accurate ZIP representations for end use loads are not always intuitive. For example, an oscillating fan is not $100 \%$ constant power and an incandescent light bulb is not $100 \%$ constant impendence. A further issue to note is that the six constants are not always positive; a condition that generally occurs in loads with active components such as switching power supplies. This does not indicate that the load generates power, just that some elements within the ZIP model generate power which is then consumed by another element. The net result is that power is consumed, but a more complicated load behavior emerges.

The ZIP values shown in Sections 2.3.1.1 through 2.3.1.9 have been used to generate composite ZIP models for time invariant loads within each residential, commercial, and industrial points of interconnection. This ensures that changes in the supply voltage due to the CVR system generate the proper change in system load. In addition to the ZIP load, loads with thermal cycles are included in Residential and Commercial loads.

\subsubsection{Loads with Thermal Cycles}

Whether a load has a thermal cycle or not, it must have the voltage dependent energy consumption of a ZIP load. If the load does have a thermal cycle, there is the added complexity of an additional control loop, which determines when the load is energized, and for how long. One of the largest load types that have a thermal cycle are Heating, Ventilation, and Air Conditioning (HVAC) systems. An equivalent thermal parameter (ETP) model is used to approximate the response of the electrical demand of the HVAC system as a function of solar 
input, temperature, humidity, voltage, and thermostatic set points [3-5]. The thermal parameters of the building are the mass of the building, which defines how much stored thermal energy is in the building, and the envelope, which defines how quickly the energy moves from inside to outside the building and can loosely be described as the insulation quality. These parameters are determined by the actual physical properties of the building, and include such things as floor area, ceiling height, aspect ratio, window types, air exchange rate, etc. Additionally, HVAC properties such as heating and cooling set points, heat type (gas, electric, or heat pump), fan power, motor losses, etc. can be defined. Figure 2.13 is a diagram of the ETP model for a residential HVAC system.

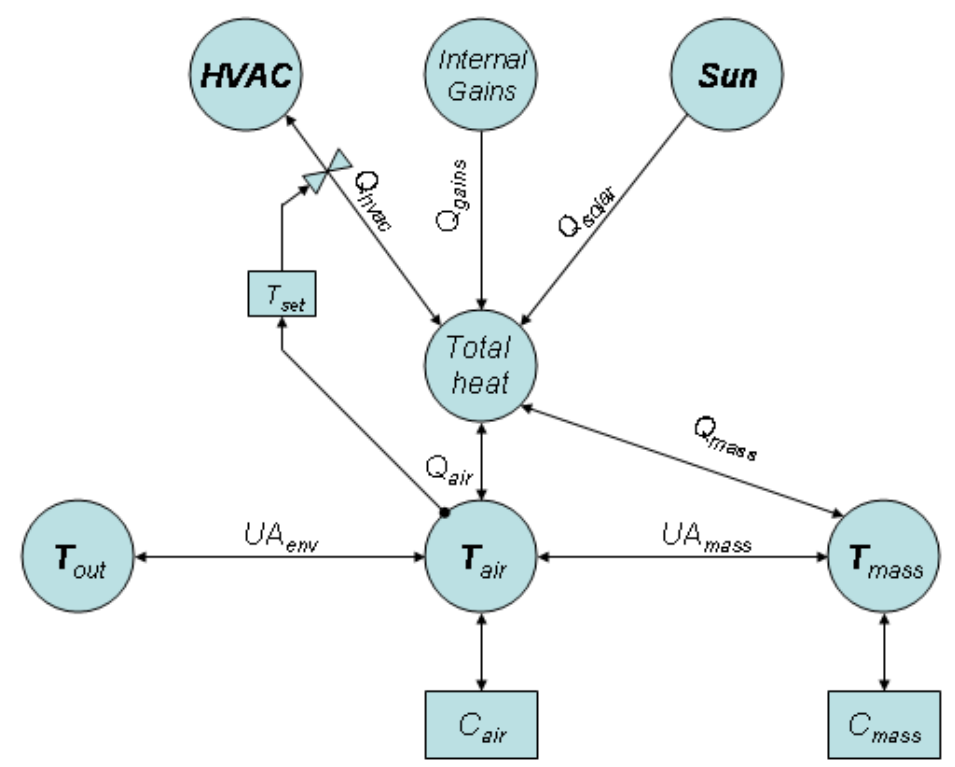

Figure 2.12: The ETP model of a residential HVAC system

where,

$C_{\text {air }}$ : air heat capacity

$C_{\text {mass }}$ : mass heat capacity

$U A_{\text {env }}$ : the gain/heat loss coefficient between air and outside

$U A_{\text {mass }}$ : the gain/heat loss coefficient between air and mass

$T_{\text {out }}$ : air temperature outside the house

$T_{\text {air }}$ : air temperature inside the house

$T_{\text {mass }}:$ mass temperature inside the house

$T_{\text {set }}$ : temperature set points of HVAC system

$Q_{\text {air }}$ : heat rate to house air

$Q_{\text {gains }}:$ heat rate from appliance waste heat

$Q_{\text {hvac }}$ : heat rate from HVAC

$Q_{\text {mass }}$ : heat rate to house mass

$Q_{\text {solar }}$ : heat rate from solar gains 
Equations (2.4) and (2.5) are the two ordinary differential equations (ODEs) which describe the heat flows shown in Figure 2.12. These equations are used to determine the thermal behavior of the house in response to the three heat sources and the user defined thermostatic set points. The solution to (2.4) and (2.5) represents the thermal behavior of the house and forms the basis for determining the electrical power consumption of the HVAC system.

$\frac{d T_{\text {air }}}{d t}=\frac{1}{C_{\text {air }}}\left[T_{\text {mass }} U A_{\text {mass }}-T_{\text {air }}\left(U A_{\text {env }}+U A_{\text {mass }}\right)+Q_{\text {air }}+T_{\text {out }} U A_{\text {env }}\right]$

$\frac{d T_{\text {mass }}}{d t}=\frac{1}{C_{\text {mass }}}\left[U A_{\text {mass }}\left(T_{\text {air }}+T_{\text {mass }}\right)+Q_{\text {mass }}\right]$

Equations (2.4) and (2.5) can also be represented by a single second order differential equation as shown in (2.6).

$a \frac{d^{2} T_{a i r}}{d t^{2}}+b \frac{d T_{a i r}}{d t}+c T_{a i r}=d$

where:

$a=\frac{C_{\text {mass }} C_{\text {air }}}{U A_{\text {mass }}}$

$b=\frac{C_{\text {mass }}\left(U A_{\text {env }}+U A_{\text {mass }}\right)}{U A_{\text {mass }}}+C_{\text {air }}$

$c=U A_{\text {env }}$

$d=Q_{\text {mass }}+Q_{\text {air }}+U A_{\text {env }} T_{\text {out }}$

\subsubsection{Load Composition for Prototypical Feeders}

Using the two load modeling methods described in Section 2.3.1 and 2.3.3 composite load models were developed for each building in the prototypical feeders. For Residential and Commercial buildings both ZIP models and Physical Models (those with thermostatic control loops and physical parameters) were used, Industrial Loads only used ZIP models. Only ZIP models were used for Industrial Loads because of the complexity required to model specific industrial processes.

For Commercial and Residential buildings, Physical Models were used for HVAC and hot water heating, and the remainder of the load was represented by a composite ZIP model using the information from Section 2.3.1. For the Physical Models there were a number of parameters 
which were sensitive with respect to climate region. For example, Regions 4 and 5 had very high levels of air conditioning, while Region 1 was relatively low. Table 2.3 shows the composition of HVAC by region for the United States as determined by EIA data, rounded to the nearest 5\% [6]. This information was used to determine what percentage of residential houses on each feeder was supplied by the various types of HVAC. As noted in Table 2.3, some residences contain natural gas as well as a heat pump. This is due to the poor efficiency of heat pumps at low temperatures where the natural gas is used for heating.

Table 2.3: HVAC Percentages by Region

\begin{tabular}{|l|c|c|c|c|c|}
\hline & $\mathrm{R} 1$ & $\mathrm{R} 2$ & $\mathrm{R} 3$ & $\mathrm{R} 4$ & $\mathrm{R} 5$ \\
\hline$\%$ Natural Gas & $60.00 \%$ & $65.00 \%$ & $50.00 \%$ & $30.00 \%$ & $40.00 \%$ \\
\hline$\%$ Heat Pump & $30.00 \%$ & $25.00 \%$ & $45.00 \%$ & $60.00 \%$ & $50.00 \%$ \\
\hline \% Electric Heat & $10.00 \%$ & $10.00 \%$ & $5.00 \%$ & $10.00 \%$ & $10.00 \%$ \\
\hline \% AC & $51.20 \%$ & $85.22 \%$ & $86.57 \%$ & $95.52 \%$ & $95.72 \%$ \\
\hline \%Differences due to the use of heats pumps for heating $>20$ degrees and gas $<20$ degrees \\
\hline
\end{tabular}

Water heaters used a similar model to the ETP model used for the thermal properties of the building. Water demand of an average home and the insulation level become the inputs for each device, and is translated into an electrical power demand as a function of time of day. The heater coil of a water heater is a resistor and reduces power demand when voltage is reduced; however, the same amount of heat energy must be put into the water to heat the water. So, while the peak demand of a single water heater is reduced by a reduction in voltage, the amount of time it stays on is increased and energy consumed is held nearly constant. For the purpose of this study, water heaters were assumed to be natural gas if the house had a natural gas connection, and therefore consumed minimal electrical energy.

\subsection{Population of Loads}

To analyze the effects of CVR, time-series simulations were performed. Because the original taxonomy feeders contained only static load models, which were representative of peak load, time varying load models were added. The static loads were replaced with time-varying thermostatic models and time-varying ZIP models. The goal was to populate the feeder with individual representative building and load models, which provided, in aggregate, a nearly identical time-varying demand at the substation level as that found in SCADA (Supervisory Control and Data Acquisition) data. This provided not only an aggregate model of the characteristics of the particular feeder, but also gave a representative model of the behavior of individual loads. While a single load populated into the model would not actually represent a particular home, the distribution of populated buildings and loads will approach the actual behavior of all the loads within the real system. This provides an understanding of how the loads and feeder would respond, in aggregate, to a reduction in voltage. 


\subsection{Transformers}

To appropriately model the effects of voltage reduction, full-load and no-load losses, and all states in between, must be properly handled. Classical transformer models are used in GridLAB$\mathrm{D}$ to capture these effects, and include series and shunt losses. This is necessary because as voltage is reduced, series losses may decrease or increase depending on the type of load, but shunt losses will always decrease. Shunt losses are always present, regardless of the demand level, so by reducing those losses, the benefits are accumulated over time.

To populate the feeder models with appropriate transformer models, standard transformer data sheets from COOPER Power Systems were used to convert no-load and full-load losses to series and shunt impedance values as a function of power rating [7]. The size of the load (load sizing will be discussed further in Load Magnitude) determined the power rating of the transformer, where the specified load from the original model was rounded up to the next smallest available standard power rating. Split-phase center-tap models were used to connect residential 1-6 and commercial 2 to $240 \mathrm{~V}$ circuits, while 1-, 2-, and 3-phase wye-wye models were used to connect commercial 1 and industrial loads to $480 \mathrm{~V}$ circuits.

\subsection{Load Magnitude and Shape}

To develop an accurate annual load profile for the feeders, each of the individual end use loads were calibrated. Relative loading across a feeder is approximately equal to that specified for the original taxonomy feeders, but with added time-dependent outdoor temperature, solar insolation, voltage, etc. However, to create a model that accurately matches provided SCADA data over the provided time interval, a number of adjustments were needed.

First, daily, weekly, and seasonal schedules were created to control thermostat set points within the homes. These were created to loosely represent a variety of customers, including those who leave their settings the same throughout a season, those who adjust the set points only on weekends, and those who adjust them on a daily or hourly basis (away versus awake versus asleep). These schedules were created from a combination of survey data and randomly distributed throughout the population of residential buildings. Adjustments were made to represent differences between seasons, between daytime and nighttime, and between weekends and weekdays, and each building contained its own unique schedule. Commercial buildings were assumed to keep more constant thermostat settings, with adjustments only made between their daytime and nighttime settings, and used similar settings for both weekdays and weekends. While the commercial settings were more constant, there were still variations between weekday and weekend to represent the behavior of commercial office buildings.

Second, hot water demand schedules were created to represent the amount of demand by hot water heaters. These were created from a combination of survey data and Department of Energy (DOE) water heater loading approximations. Events related to showers, dishwashers, hand washing, and clothes washers were simulated to represent the demand on the hot water heater. Once again, each building with an electric hot water heater (buildings supplied by gas lines were assumed to have gas water heaters) contained its own unique water demand schedule. 
These two loads were selected as physical models (as opposed to generic ZIP models) due to their large impact on the demand of a residential home. Capturing the actual state driven behavior, as opposed to average behavior, was essential in understanding the effects of voltage reduction, since the average behavior has not been fully quantified during voltage reduction operations. To capture the effects of smaller appliances, time-varying ZIP models were created. The time dependent effect was created using a library of yearly load shapes, containing seasonal, weekly, daily, and hourly variations at 15-minute intervals, most created from raw SCADA data. Large commercial and industrial loads were created using a similar method. Power factor and ZIP fractions were assigned from available anecdotal information, including measured laboratory data and previous CVR studies.

At each spot load location from the original feeder model, the load was replaced with a combination of building and ZIP load models. By varying the relative number of building models to the number of ZIP models, then varying their relative magnitudes, a reasonable approximation could be found that matched SCADA data for the entirety of a year. By fitting data on approximately 6-12 days per year per feeder, it was found that overall difference between simulated and actual demand could be limited to approximately $5 \%$ of the total demand at all times throughout the year, except during times of topological changes (for example, if a large amount of load was shifted from one circuit to another).

\subsection{Method of Conservation Voltage Reduction}

CVR is not a new technology. There have been numerous proposed methods [8-14], numerous studies of deployed systems [13-20], and many vendors offer CVR based systems [23-26]. For the purposes of this analysis, a CVR scheme that has been published in the IEEE Transactions on Power Systems and is openly available has been selected [11].

In the selected system, there are two major functions: reactive power control and voltage optimization. The reactive power control operates the shunt capacitors on the distribution feeder in order to improve the power factor at the substation. The voltage optimization operates the sub-station voltage regulator in order to control the system voltage as measured at the End Of Line (EOL) measurements. For the selected CVR system, control of additional downstream voltage regulators is not supported. Control of multiple voltage regulators on a distribution feeder is provided in modern, commercially available CVR products, and will further increase the performance. 


\section{Individual Prototypical Feeder Results}

To estimate the national benefit of CVR, the Taxonomy of Prototypical distribution feeders developed at PNNL for the Modern Grid Initiative (MGI), now the Modern Grid Strategy (MGS), was used. Each of the 24 prototypical distribution feeders was populated with ZIP models and full Equivalent Thermal Parameter (ETP) models for residential and commercial HVAC, which included their associated secondary distribution systems. The populated feeders were then simulated in a "traditional" voltage control scheme for an entire year at a 1 minute time step. The total energy consumed was then calculated for: the total feeder, the residential loads, the commercial and industrial loads, and the various system losses. Additionally, a set of End of Line (EOL) voltages was recorded for each phase. The EOL point was determined based on the low voltage primary node at maximum system load. This voltage was then assumed to be lowest voltage point on the system at any given time. The simulation was then rerun with the exact same feeders and load conditions, but with the CVR system operating. The difference in energy consumption was then examined.

The two key benefits of CVR are peak load reduction and reduction in annual energy consumption. When the peak load is reduced, fewer generating units are required, especially costlier peaking units, while annual energy reduction requires less primary fuel to be consumed. The following sections examine the peak load reduction and reduction in annual energy consumption for each of the individual prototypical feeders.

\subsection{Peak Load Reduction}

Figure 3.1 shows the peak demand change in $\mathrm{kW}$, while Figure 3.2 shows the peak demand change as a percent of total feeder loading, for each of the prototypical feeders. By observation, it can be seen that all but two of the prototypical feeders sees a reduction in the peak demand when CVR is in operation. The one feeder experiencing a noticeable increase in the peak demand, R1-25.00-1, is a long feeder that already has a low end of line voltage. As a result, when CVR begins to regulate, it actually raises the voltage during the peak load, resulting in a higher peak demand. This is not an unexpected occurrence because many feeders in the United States are long, rural feeders, where significant capital investment in infrastructure is not cost effective. R1-25.00-1 is a higher voltage $25 \mathrm{kV}$ class feeder, but with a peak load of only 2,300 $\mathrm{kW}$, from Figure 3.3. The low voltage is due to the small cross section of conductor that is used on this feeder, representative of a cost savings effort for a lightly loaded rural feeder. With reconductoring or a mid-line regulator, peak reductions would be observed, but this may not be a cost effective measure.

With the exception of R1-25.00-1, and to a much less extent, R4-25.00-1, each of the taxonomy feeders experiences a noticeable reduction in peak load, between $0.5 \%$ and $4.0 \%$. One point to notice is that while the percent reduction in peak load is similar among many feeders, the $\mathrm{kW}$ reductions vary significantly; this is primarily due to the loading of the different feeders. The reduction in energy consumed is a function of two factors: the first is how many volts the 
average voltage can be reduced, and the second is the amount of load being supplied by the feeder. The ideal feeder for CVR would be a heavily loaded feeder that is able to support a significant reduction in voltage.

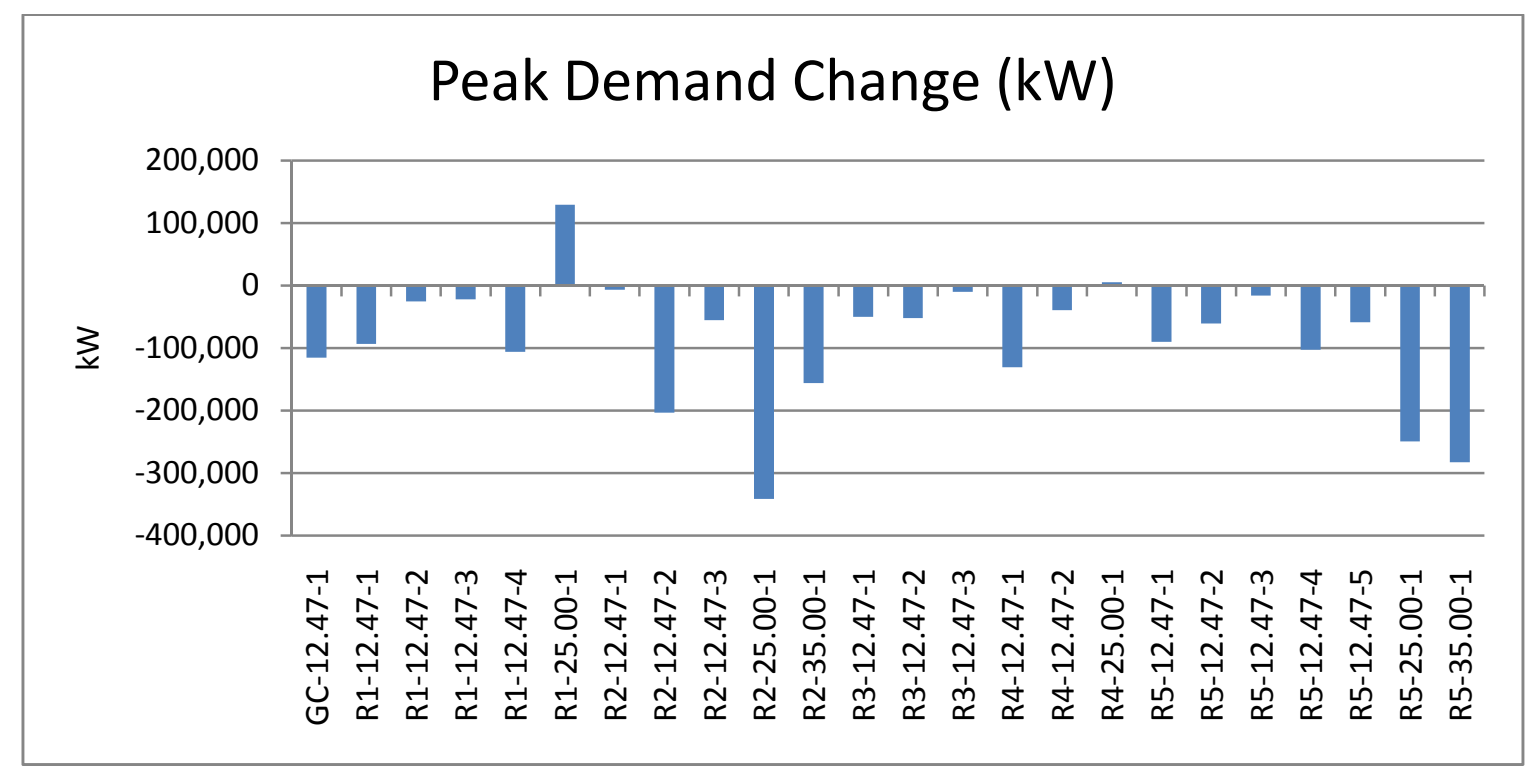

Figure 3.1: Peak Demand Change (kWh) by Taxonomy Feeder

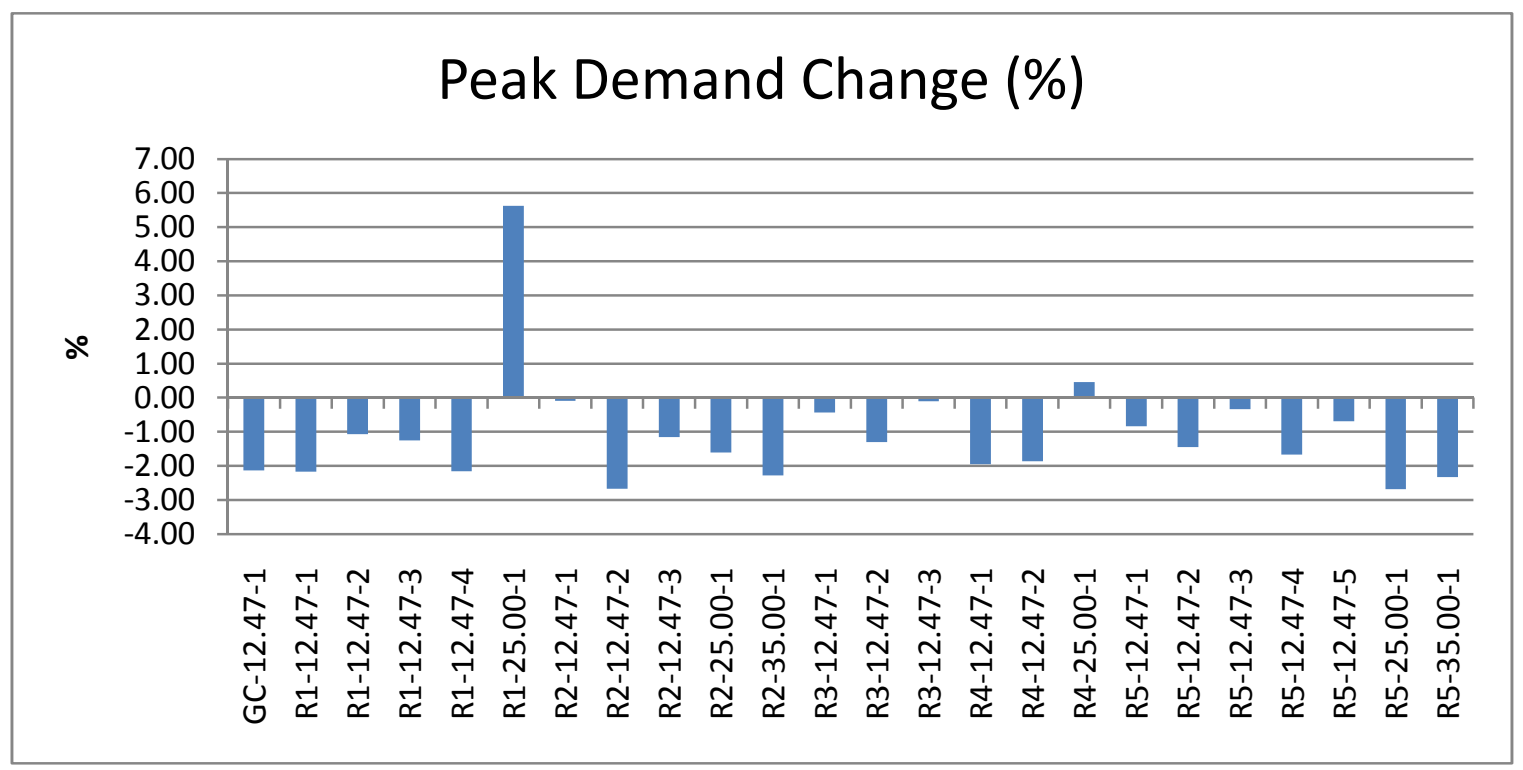

Figure 3.2: Peak Demand Change (\%) by Taxonomy Feeder 


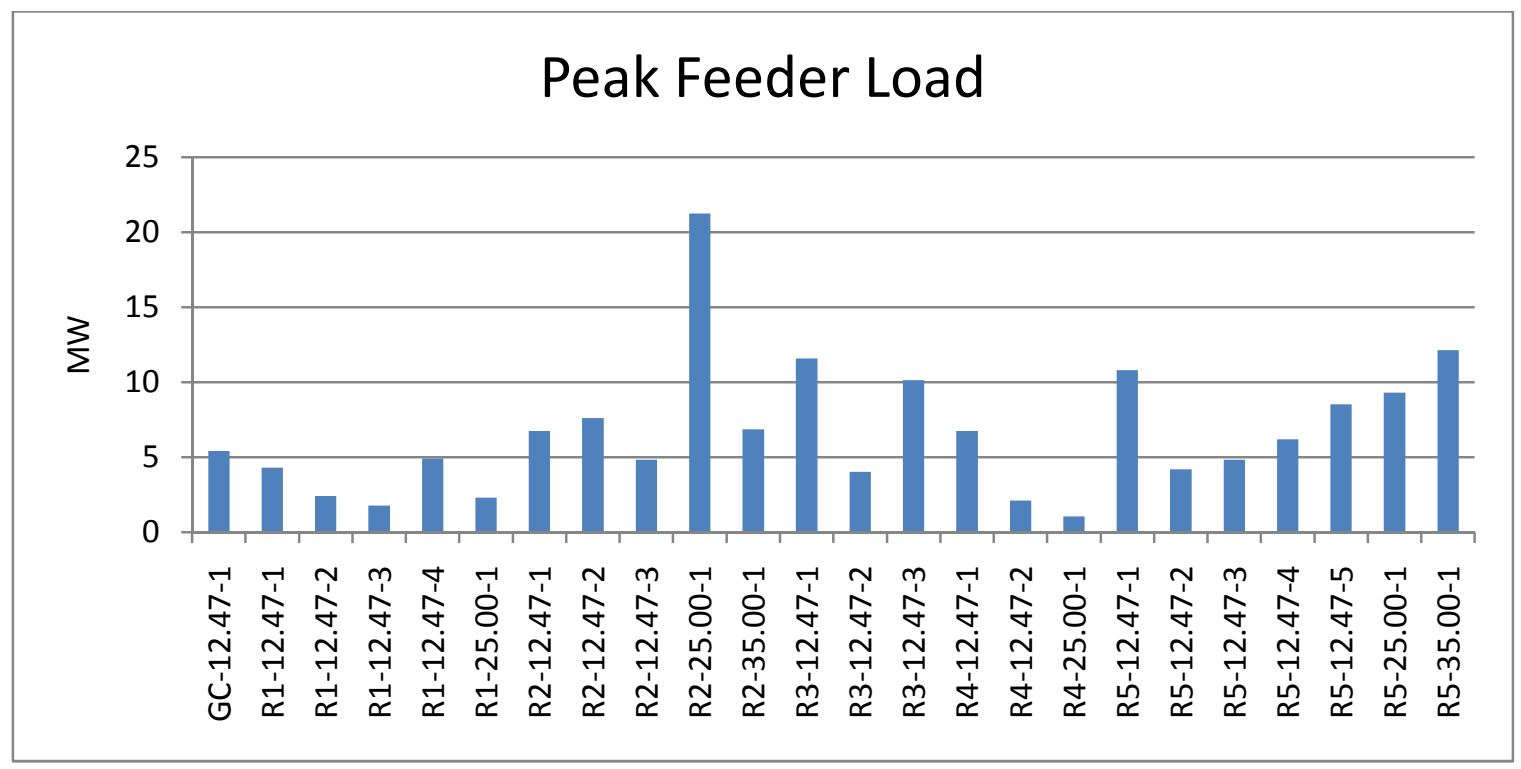

Figure 3.3: Peak loading (MW) by Taxonomy Feeder

For CVR to be effective it must be possible to reduce the average voltage along the feeder; an inability to do this is why R1-25.00-1 underwent an increase in peak demand. Figures 3.4 and 3.5 show the annual minimum voltage at the end of line measurements when operating without CVR, and with CVR. Figures 3.6 and 3.7 show the average annual voltage as measured at the end of line points when operating without CVR, and with CVR respectively. The CVR system of Section 2.7 operates to ensure that the EOL measurements are never below $118 \mathrm{~V}+/-1 \mathrm{~V}$, effectively ensuring that the end of line measurements are greater than 117V. From Figure 3.5, it is clear that the voltage does drop below 117 volts on almost every feeder. This is a transient condition and the voltage is quickly raised. Figure 3.7 shows the average voltage and it is clear that the CVR system is regulating to an $118 \mathrm{~V}$ average. By comparing the without and with CVR average voltages, it can be seen that the average voltage at the end of line points is reduced. While this is a reasonable indicator of the effectiveness of CVR on a particular feeder, it does not take into consideration system load, or the fact that this is the voltage as measured at one point in the system. A limitation with the majority of current CVR schemes is that they rely on remote measurements from a handful of locations, and assume that they are representative of the entire system.

In general, it is clear that CVR has the potential to reduce peak demand on distribution feeders. The ability to reduce the peak demand of a feeder could be further increased through upgrades such as feeder reconductoring and installation of downstream voltage regulators. 


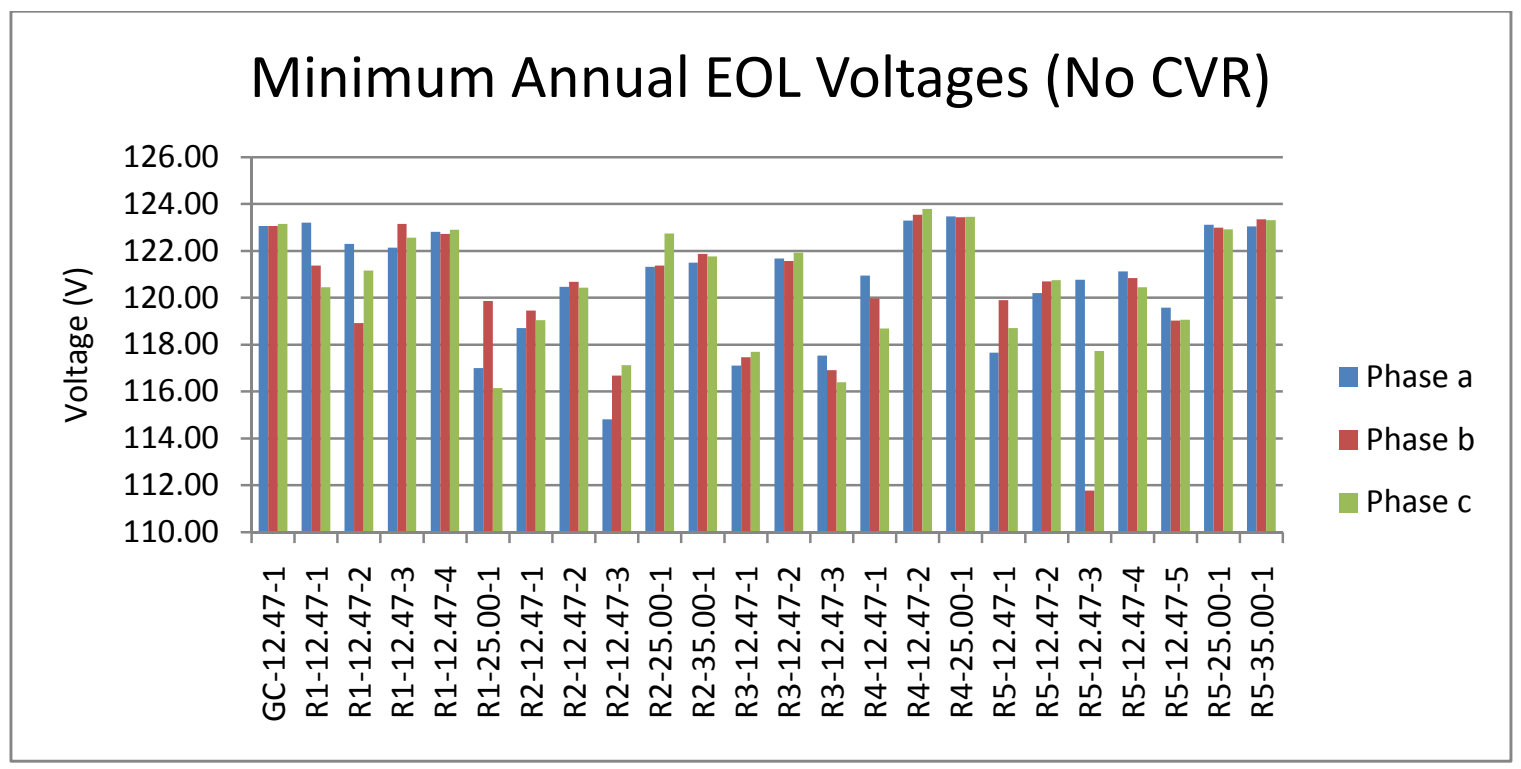

Figure 3.4: Minimum Annual EOL Voltages for Prototypical Feeders with CVR Off

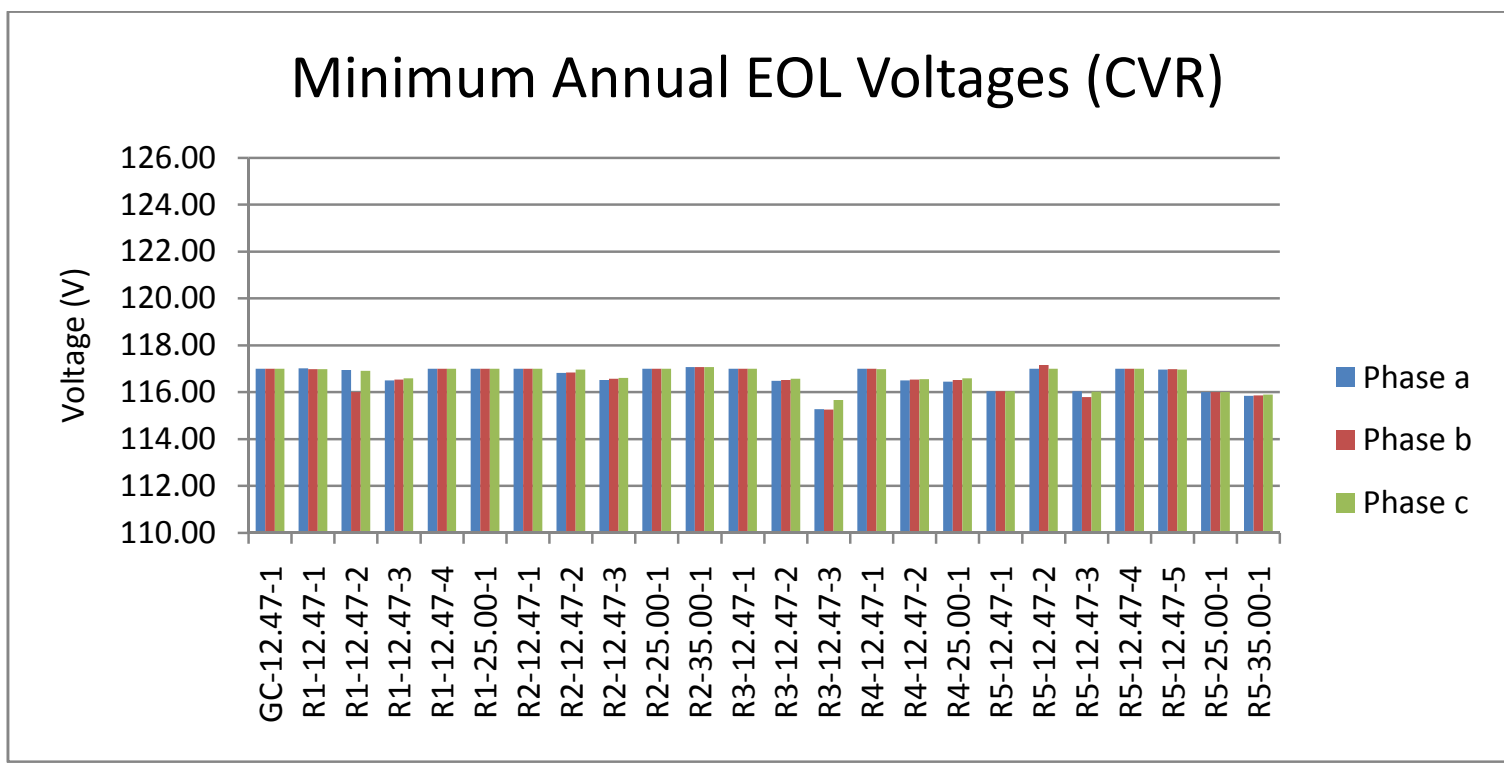

Figure 3.5: Minimum Annual EOL Voltages for Prototypical Feeders with CVR On 


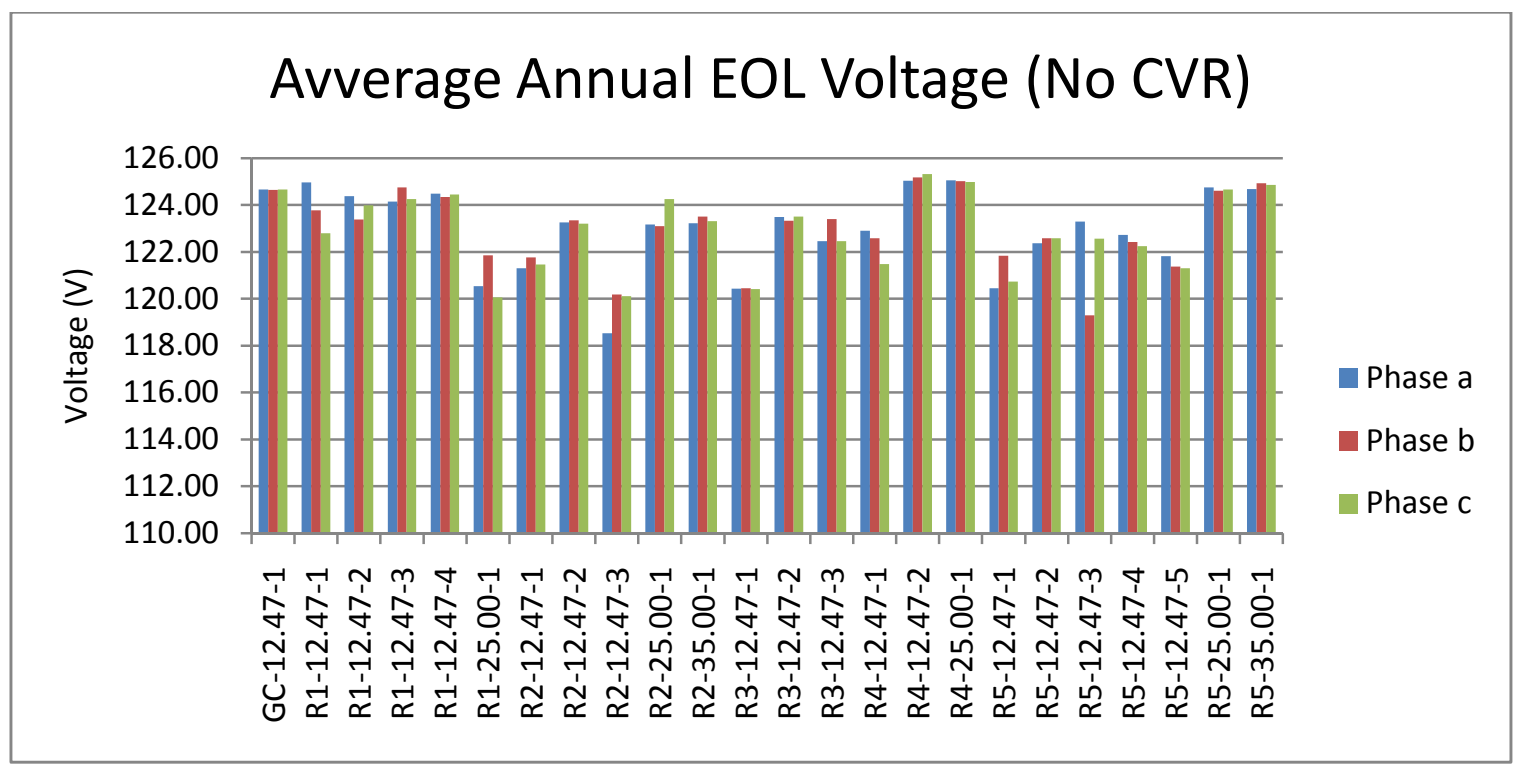

Figure 3.6: Average Annual EOL Voltages for Prototypical Feeders with CVR Off

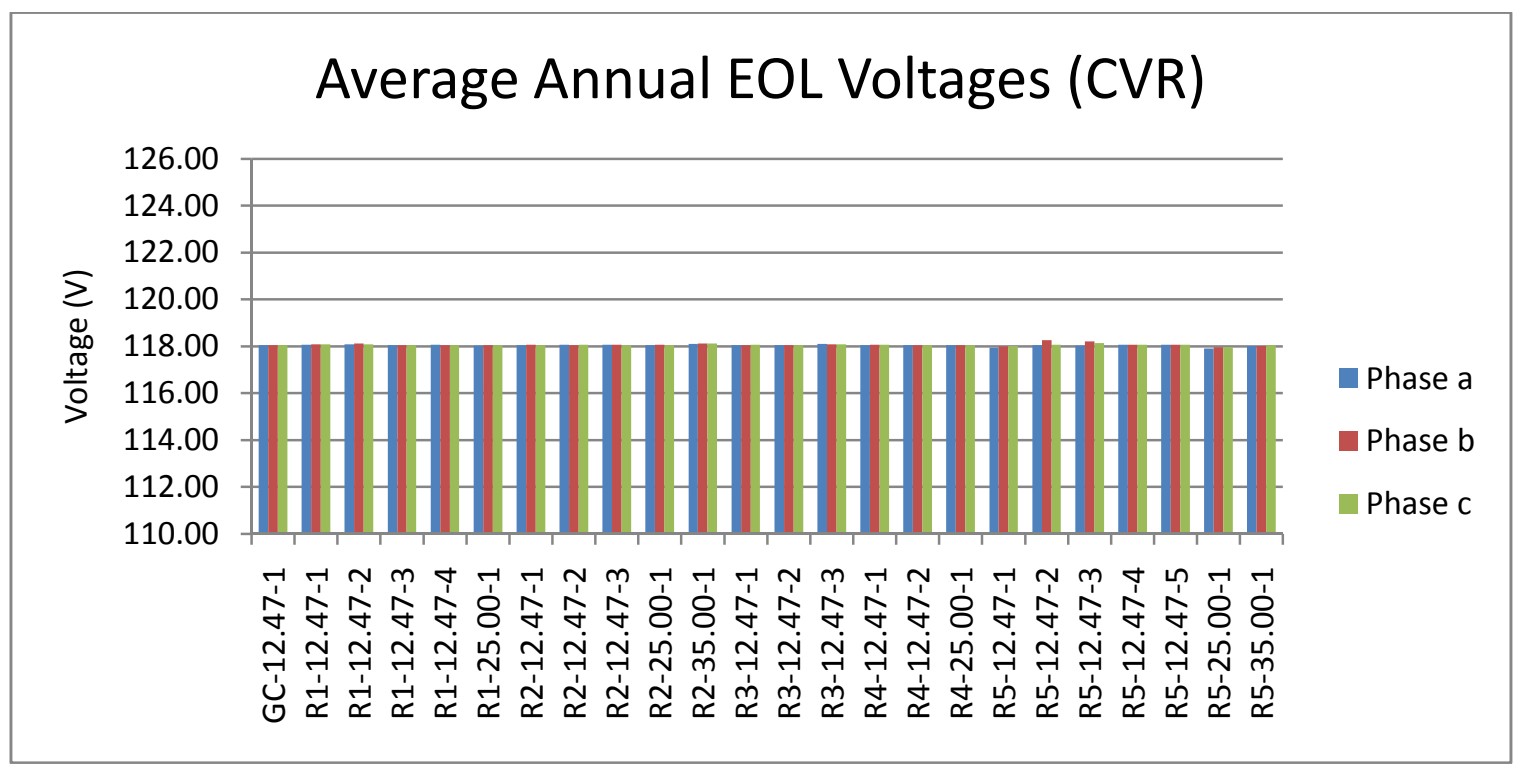

Figure 3.7: Average Annual EOL Voltages for Prototypical Feeders with CVR On

\subsection{Reduced Annual Energy Consumption}

While the last section focused on the ability of CVR to provide a power reduction during the peak day of the year, this section will focus on the ability of CVR to provide continued energy reduction over the course of an entire year. Figures 3.8 and 3.9 are similar to Figures 3.1 and 3.2 except that they show that annual reduction in energy as opposed to the peak demand 
reduction. As with peak demand, the ability of CVR to reduce the annual energy consumption is evident, as shown in Figures 3.8 and 3.9. Once again R1-25.00-1 is the notable exception in that the annual energy consumption increases when CVR is in operation. As with peak demand, the inability of CVR to reduce annual energy consumption is due to the particular design of the feeder. If capital improvements were made, superior performance would be expected.

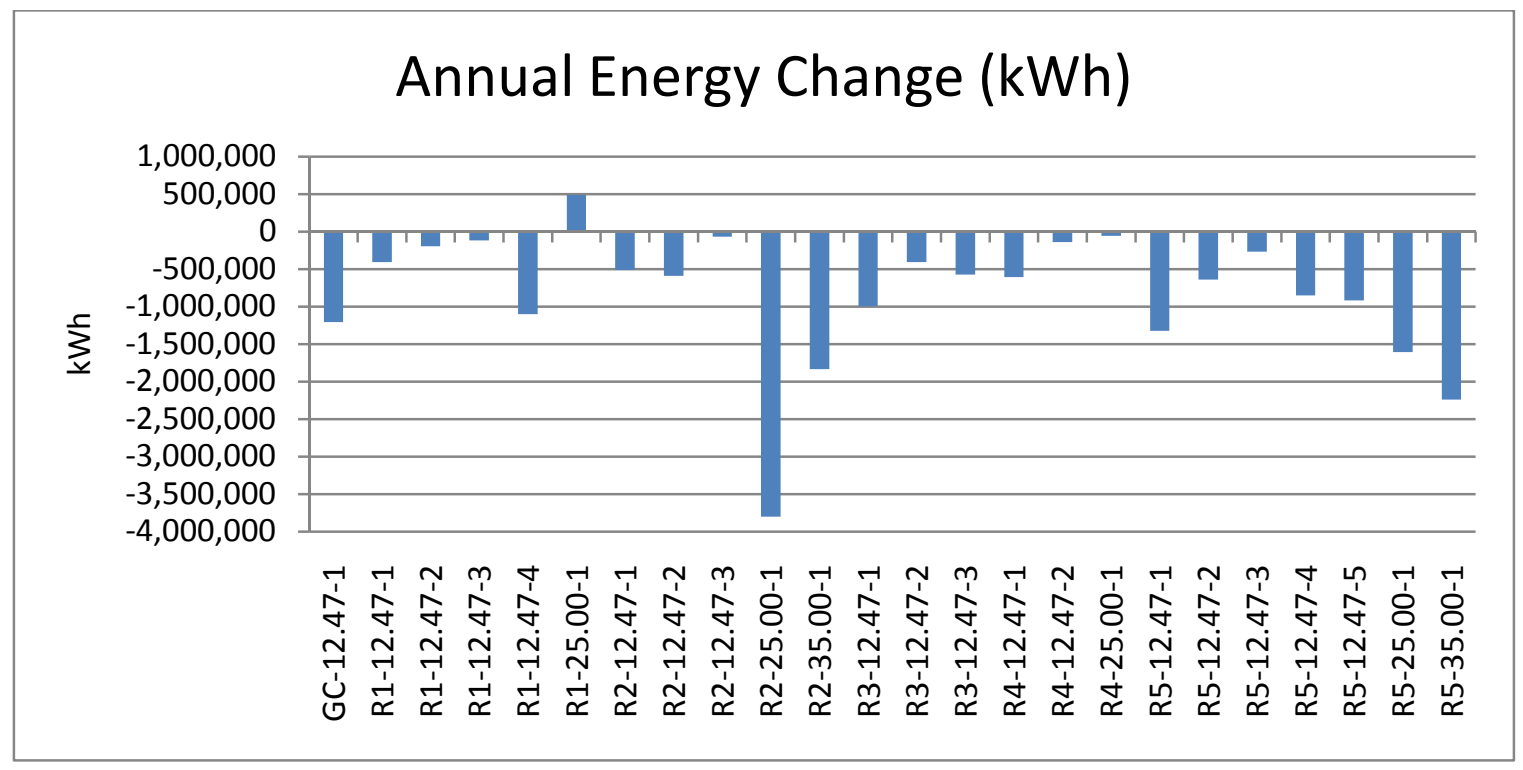

Figure 3.8: Annual Energy Change (kWh) by Taxonomy Feeder

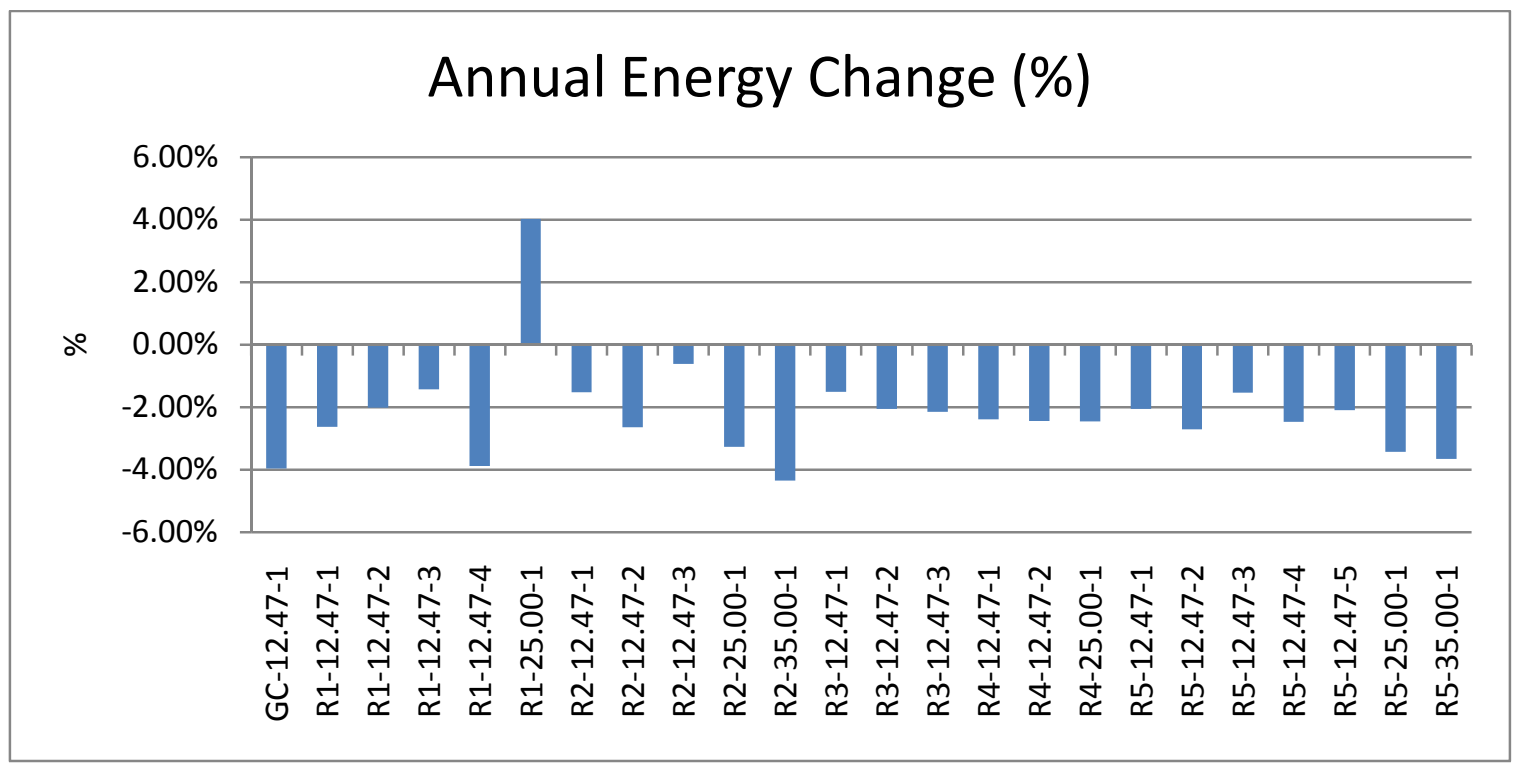

Figure 3.9: Annual Energy Change (\%) by Taxonomy Feeder

Figures 3.1 through 3.9 have given high level information about the performance of CVR on 
the various prototypical feeders. Since GridLAB-D was use to perform the analysis, there is a substantial amount of additional information that can be examined. For each of the prototypical distribution feeders this report will examine the following six (6) plots:

1) Total Energy Change $(\mathrm{kWh})$

2) Total Energy Change (\%)

3) Total Load Change (kWh)

4) Total Load Change (\%)

5) Total Loss Change (kWh)

6) Total Loss Change (\%)

Total Energy Change, plots 1 and 2, represents the change in energy as measured at the output of the feeder regulator. Total Load Change, plots 3 and 4, represents the change in energy of the end use loads, as measured at the customer point of interconnections. Total Loss Change, plots 5 and 6 , represents the change in energy of the system losses, which include: overhead lines losses, underground line losses, transformer loses, and triplex line losses. Total Energy Change, plot 1, is the sum of Total Load Change, plot 3, and Total Loss Change, plot 5. Figures 3.10 through 3.15 show the six plots for feeder GC-12.47-1. Additionally, Figure 3.16 is a comparison of the change in total load and change in total losses.

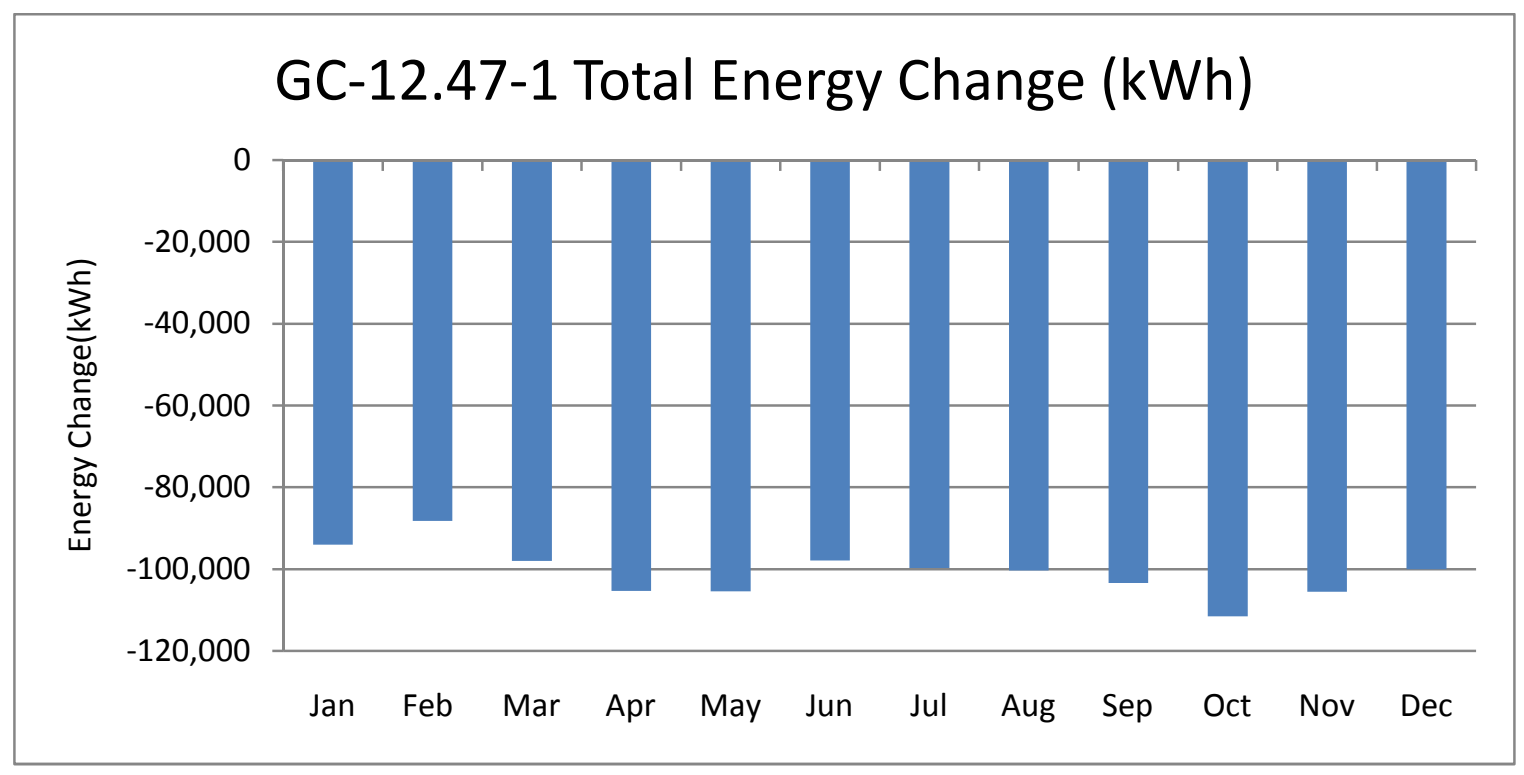

Figure 3.10: GC-12.47-1 Total Energy Change (kWh) 


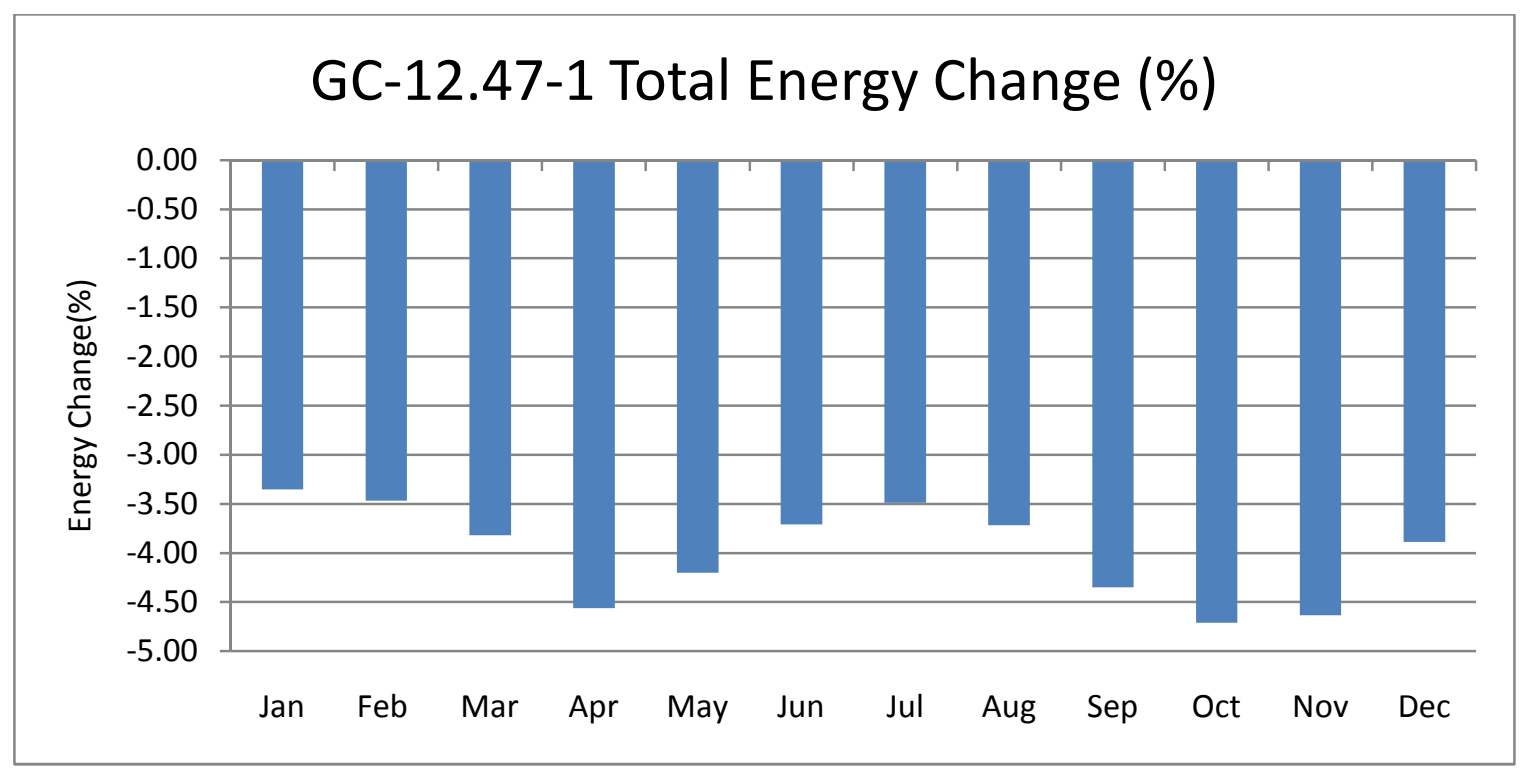

Figure 3.11: GC-12.47-1 Total Energy Change (\%)

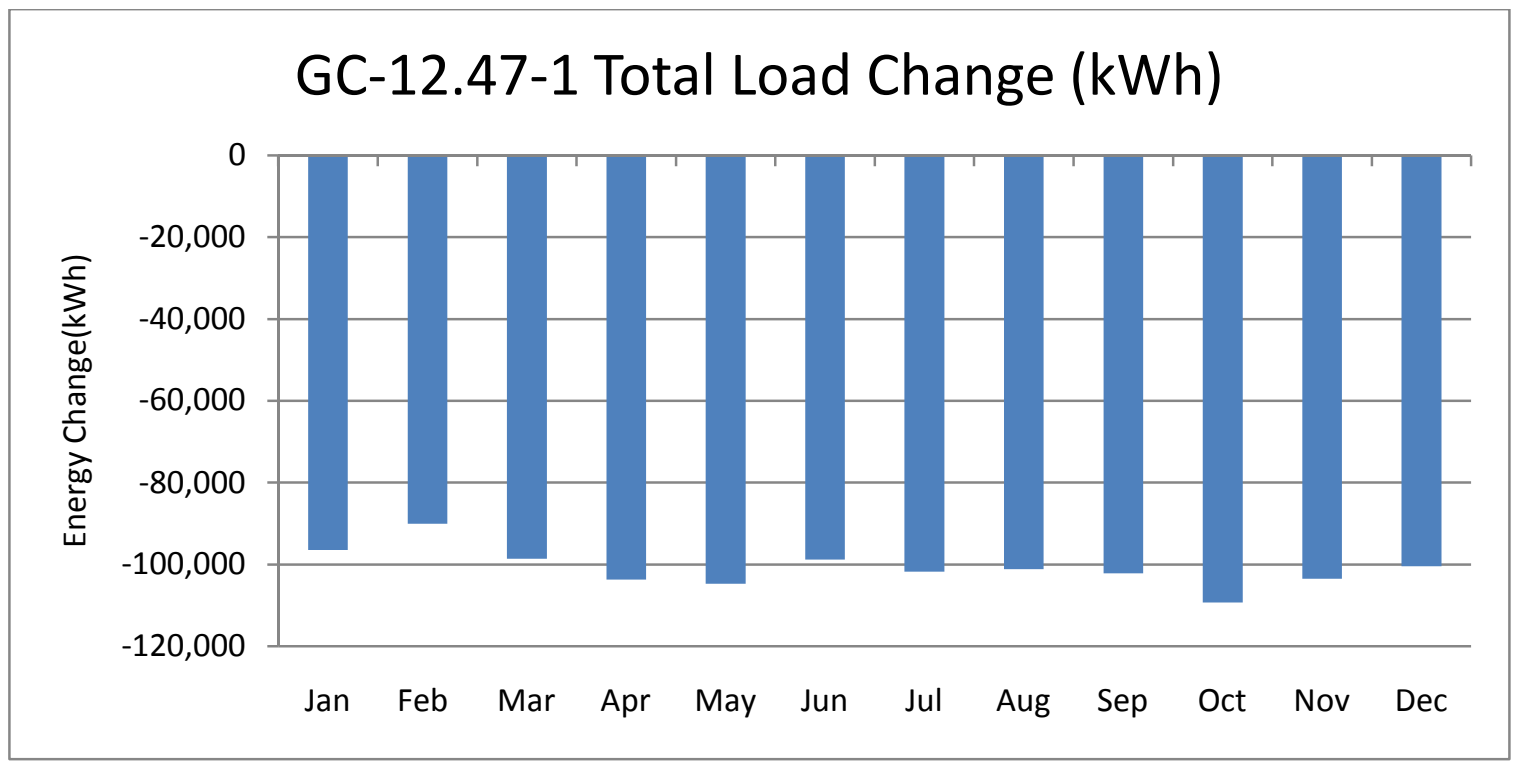

Figure 3.12: GC-12.47-1 Total Load Change (kWh) 


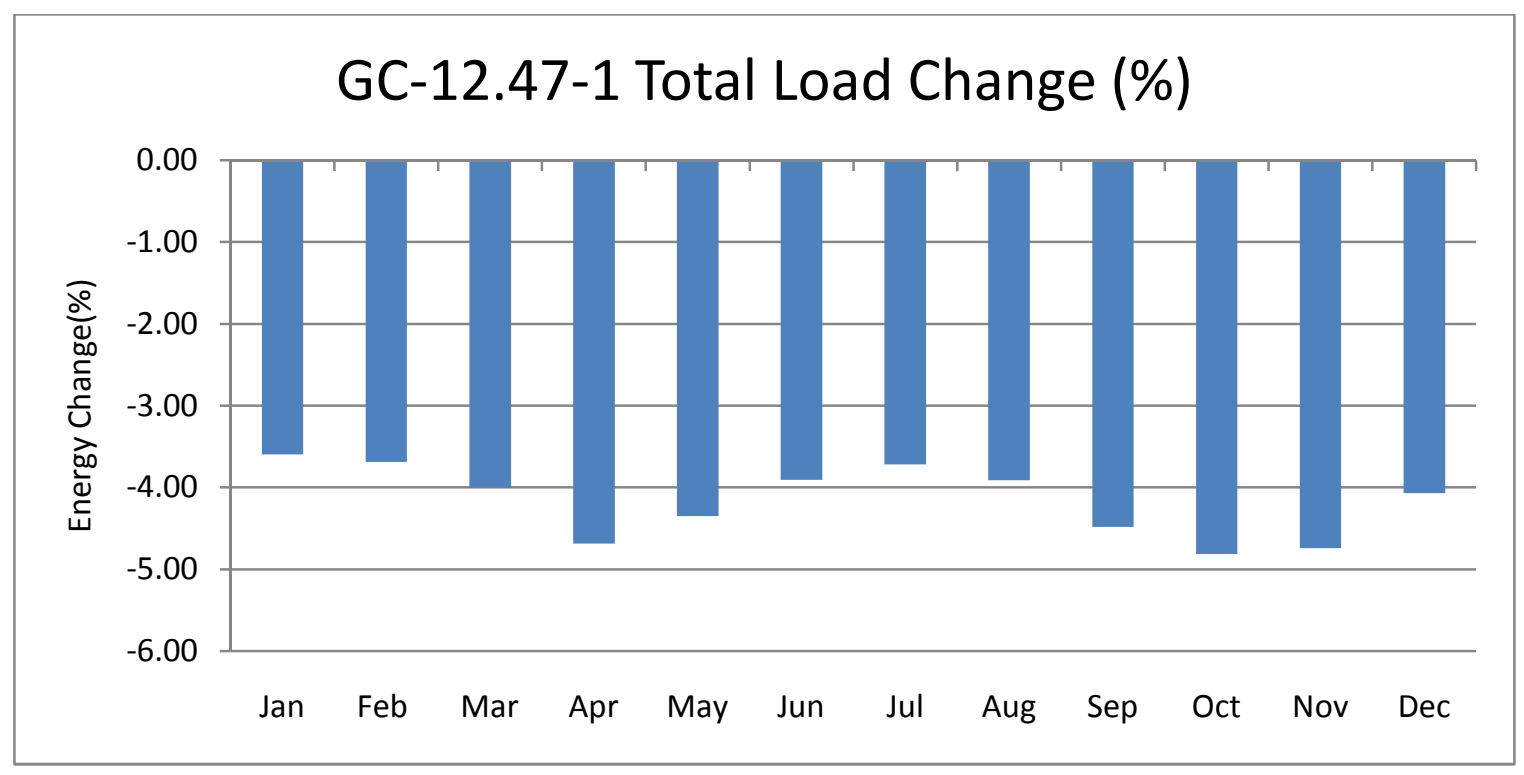

Figure 3.13: GC-12.47-1 Total Load Change (\%)

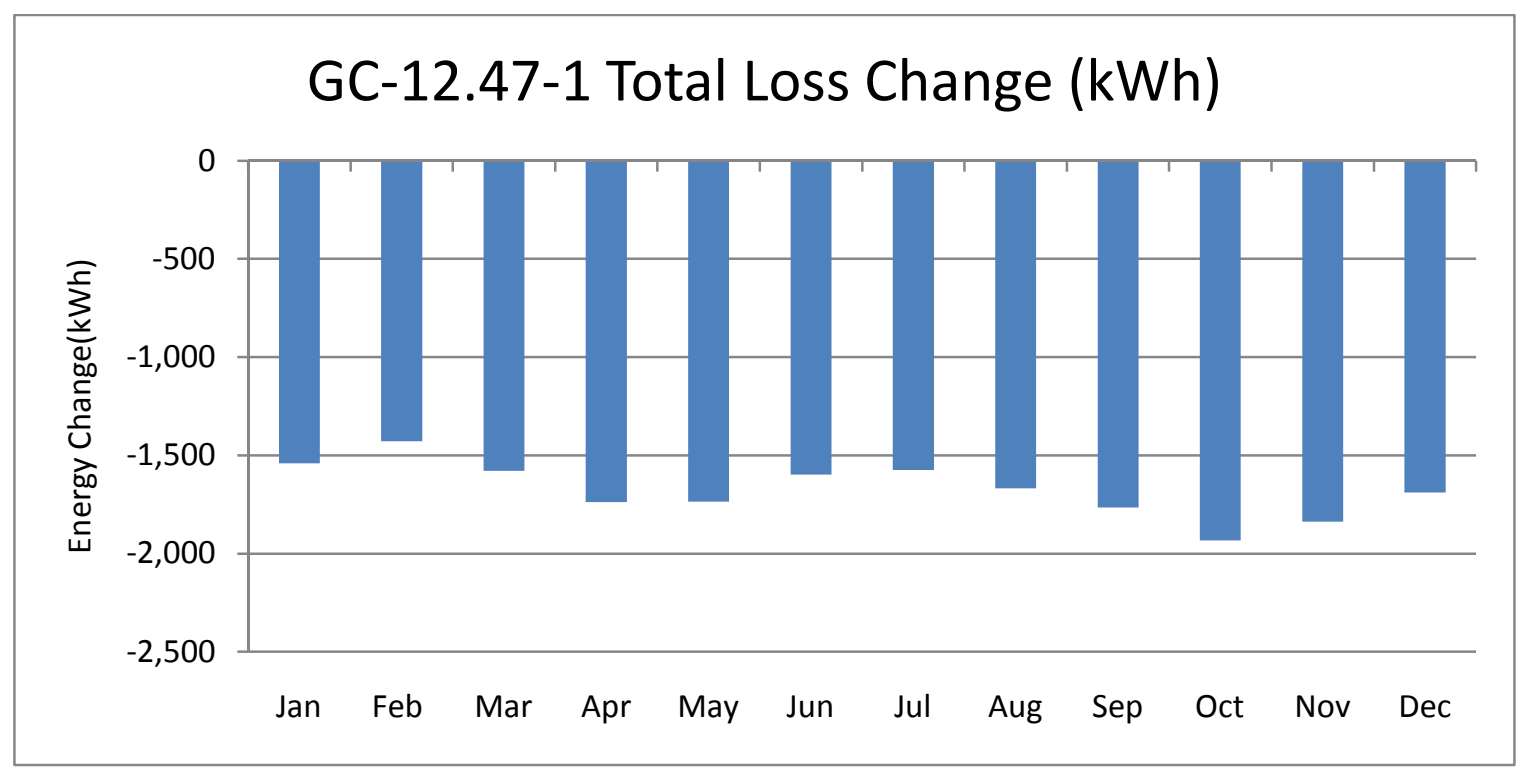

Figure 3.14: GC-12.47-1 Total Loss Change (kWh) 


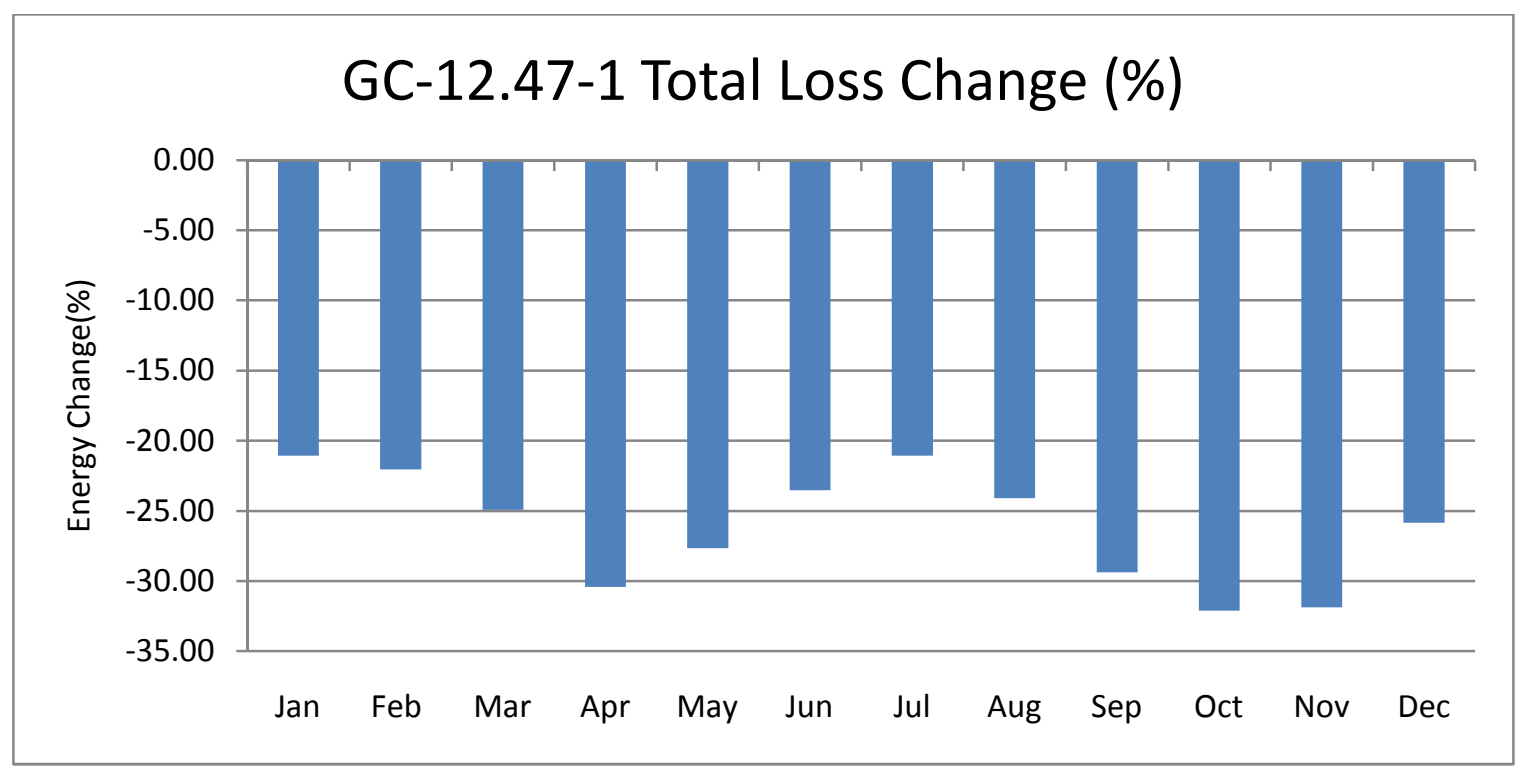

Figure 3.15: GC-12.47-1 Total loss Change (\%)

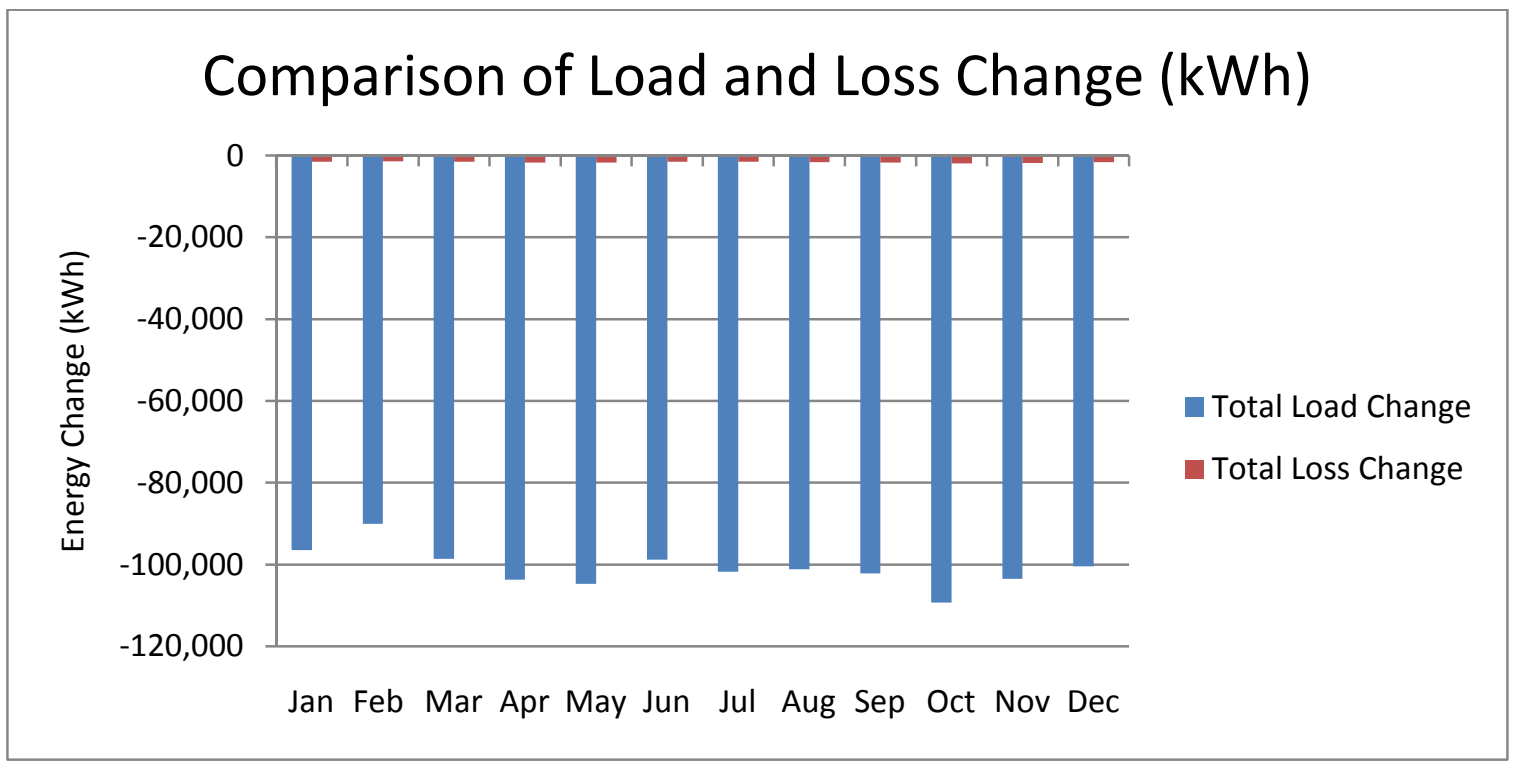

Figure 3.16: Comparison of Load and Loss Change (kWh)

One key observation from Figure 3.16, which can be seen on every feeder, is the difference in scale between the total load change, plot 3 , and the total loss change, plot 5 . Without exception, the reduction in load accounts for the vast majority of the change in energy consumption. In general, when CVR is in operation $98 \%-99 \%$ of the change in energy consumption occurs in the end use loads, while only $1 \%-2 \%$ of the reduction in energy consumed can be attributed to losses. Reduction in systems losses is not a significant benefit of CVR.

Because of the large number of prototypical distribution feeders it is not practical to place six plots for each feeder in the main body of the report. Section 6 of this report is Appendix I and it 
provides detailed plots for each feeder on a month by month basis, similar to Figures 3.10 through 3.15 . 


\section{Extrapolation to a National Level}

While section three examined the detailed effects of CVR on the prototypical distribution feeders, it is the extrapolation of these results to a national level that is of the most interest. The taxonomy of prototypical feeders was chosen for this work because of its ability to readily extrapolate results to a national level [1]; each of the 24 prototypical feeders is representative of a number of feeders within the climate regions shown in Figure 2.1.

Table 4.1 is a reprint of Table 9 from [1], which gives an estimate of the number of feeders in each climate region that are represented by the various prototypical feeders. For example, R212.47-3 is representative of 3,000 feeders within region 2 . Therefore, the results of CVR analysis on R2-12.47-3 can be assumed to represent similar behavior on 3,000 distribution feeders. This method can be used to extrapolate the results of analysis of the 24 prototypical feeders to the national level.

Table 4.1: Prototypical Feeder Weighting Factors

\begin{tabular}{|c|c|c|c|c|}
\hline Region & Feeder & $\mathrm{kV}$ & $\#$ of feeders & $\%$ within a region \\
\hline \multirow{6}{*}{ Region 1} & R1-12.47-1 & 12.5 & 2,200 & $20.56 \%$ \\
\hline & R1-12.47-2 & 12.47 & 2,500 & $23.36 \%$ \\
\hline & R1-12.47-3 & 12.47 & 2,000 & $18.69 \%$ \\
\hline & R1-12.47-4 & 12.47 & 1,800 & $16.82 \%$ \\
\hline & R1-25.00-1 & 24.9 & 1,200 & $11.21 \%$ \\
\hline & GC-12.47-1 & 12.47 & 1,000 & $9.35 \%$ \\
\hline \multirow{6}{*}{ Region 2} & R2-12.47-1 & 12.47 & 3,500 & $18.72 \%$ \\
\hline & R2-12.47-2 & 12.47 & 3,200 & $17.11 \%$ \\
\hline & $\mathrm{R} 2-12.47-3$ & 12.47 & 3,000 & $16.04 \%$ \\
\hline & R2-25.00-1 & 24.9 & 3,500 & $18.72 \%$ \\
\hline & R2-35.00-1 & 34.5 & 4,000 & $21.39 \%$ \\
\hline & GC-12.47-1 & 12.47 & 1,500 & $8.02 \%$ \\
\hline \multirow{4}{*}{ Region 3} & R3-12.47-1 & 12.47 & 1,500 & $30.00 \%$ \\
\hline & R3-12.47-2 & 12.47 & 1,500 & $30.00 \%$ \\
\hline & R3-12.47-3 & 12.47 & 1,000 & $20.00 \%$ \\
\hline & GC-12.47-1 & 12.47 & 1,000 & $20.00 \%$ \\
\hline \multirow{4}{*}{ Region 4} & R4-12.47-1 & 13.8 & 14,000 & $33.14 \%$ \\
\hline & $\mathrm{R} 4-12.47-2$ & 12.5 & 15,000 & $35.50 \%$ \\
\hline & R4-25.00-1 & 24.9 & 12,500 & $29.59 \%$ \\
\hline & GC-12.47-1 & 12.47 & 750 & $1.78 \%$ \\
\hline \multirow{6}{*}{ Region 5} & R5-12.47-1 & 13.8 & 400 & $8.79 \%$ \\
\hline & R5-12.47-2 & 12.47 & 600 & $13.19 \%$ \\
\hline & R5-12.47-3 & 13.8 & 650 & $14.29 \%$ \\
\hline & R5-12.47-4 & 12.47 & 500 & $10.99 \%$ \\
\hline & R5-12.47-5 & 12.47 & 450 & $9.89 \%$ \\
\hline & R5-25.00-1 & 22.9 & 450 & $9.89 \%$ \\
\hline
\end{tabular}




\begin{tabular}{|c|c|c|c|} 
R5-35.00-1 & 34.5 & 500 & $10.99 \%$ \\
\hline GC-12.47-1 & 12.47 & 1,000 & $21.98 \%$ \\
\hline
\end{tabular}

Table 4.2 shows the results of the individual prototypical feeders multiplied by the number of feeders from Table 4.1 in order to determine the regional level impact of CVR on total energy change. For example, Feeder R1-12.47-1 showed an annual reduction of $407 \mathrm{kWh}$, which applied to 2,200 feeders, yields a reduction of $897 \mathrm{MWh}$ in Region 1.

Table 4.2: Prototypical Feeder Regional Results

\begin{tabular}{|c|c|c|c|c|}
\hline & & $\begin{array}{c}\text { Individual Level kWh } \\
\text { Change }\end{array}$ & \# & $\begin{array}{l}\text { Regional Level } \\
\text { MWh Change }\end{array}$ \\
\hline \multirow{6}{*}{ Region 1} & R1-12.47-1 & $-407,868$ & 2,200 & $-897,310$ \\
\hline & R1-12.47-2 & $-195,907$ & 2,500 & $-489,768$ \\
\hline & R1-12.47-3 & $-117,830$ & 2,000 & $-235,661$ \\
\hline & R1-12.47-4 & $-1,102,200$ & 1,800 & $-1,983,960$ \\
\hline & R1-25.00-1 & 485,613 & 1,200 & 582,735 \\
\hline & GC-12.47-1 & $-1,209,248$ & 1,000 & $-1,209,248$ \\
\hline \multirow{6}{*}{ Region 2} & R2-12.47-1 & $-510,276$ & 3,500 & $-1,785,966$ \\
\hline & R2-12.47-2 & $-588,283$ & 3,200 & $-1,882,506$ \\
\hline & R2-12.47-3 & $-67,624$ & 3,000 & $-202,872$ \\
\hline & R2-25.00-1 & $-3,800,280$ & 3,500 & $-13,300,981$ \\
\hline & R2-35.00-1 & $-1,835,717$ & 4,000 & $-7,342,868$ \\
\hline & GC-12.47-2 & $-1,209,248$ & 1,500 & $-1,813,872$ \\
\hline \multirow{4}{*}{ Region 3} & R3-12.47-1 & $-996,426$ & 1,500 & $-1,494,639$ \\
\hline & R3-12.47-2 & $-408,226$ & 1,500 & $-612,339$ \\
\hline & R3-12.47-3 & $-573,844$ & 1,000 & $-573,844$ \\
\hline & GC-12.47-3 & $-1,209,248$ & 1,000 & $-1,209,248$ \\
\hline \multirow{4}{*}{ Region 4} & R4-12.47-1 & $-609,469$ & 750 & $-457,102$ \\
\hline & R4-12.47-2 & $-138,193$ & 14,000 & $-1,934,695$ \\
\hline & R4-25.00-1 & $-56,084$ & 15,000 & $-841,262$ \\
\hline & GC-12.47-4 & $-1,209,248$ & 12,500 & $-15,115,599$ \\
\hline \multirow{8}{*}{ Region 5} & R5-12.47-1 & $-1,324,791$ & 1,000 & $-1,324,791$ \\
\hline & R5-12.47-2 & $-639,862$ & 400 & $-255,945$ \\
\hline & R5-12.47-3 & $-270,192$ & 600 & $-162,115$ \\
\hline & R5-12.47-4 & $-851,251$ & 650 & $-553,313$ \\
\hline & R5-12.47-5 & $-919,006$ & 500 & $-459,503$ \\
\hline & R5-25.00-1 & $-1,609,031$ & 450 & $-724,064$ \\
\hline & R5-35.00-1 & $-2,238,386$ & 450 & $-1,007,274$ \\
\hline & GC-12.47-5 & $-1,209,248$ & 500 & $-604,624$ \\
\hline
\end{tabular}


If the analyzed CVR scheme were applied to all of the non-networked distribution feeders in the United States, with the exception of feeders represented by R1-25.00-1, the reduction in energy consumption would be approximately 6,500 MWyr; which is nearly the output of Grand Coulee Dam if operated at nameplate capacity for the entire year.

As with most technologies it is necessary to use discretion when deploying CVR. From Section 3 it is clear that while some feeders do show improvement, it is minimal and would not warrant the capital expenditure of a CVR system. Figure 4.1 is a plot showing the percent total benefit as a function of percent of total number of feeders. For example, it can be seen that if CVR is deployed on $40 \%$ of the total feeders in the United States, over $80 \%$ of the potential benefit can be achieved. In fact, the individual feeder results from Section 3 as well as Figure 4.1 show that CVR deployment on only the heavily loaded, higher voltage feeders yields $37 \%$ of the total benefit and only requires $10 \%$ of the total feeders to deploy CVR.

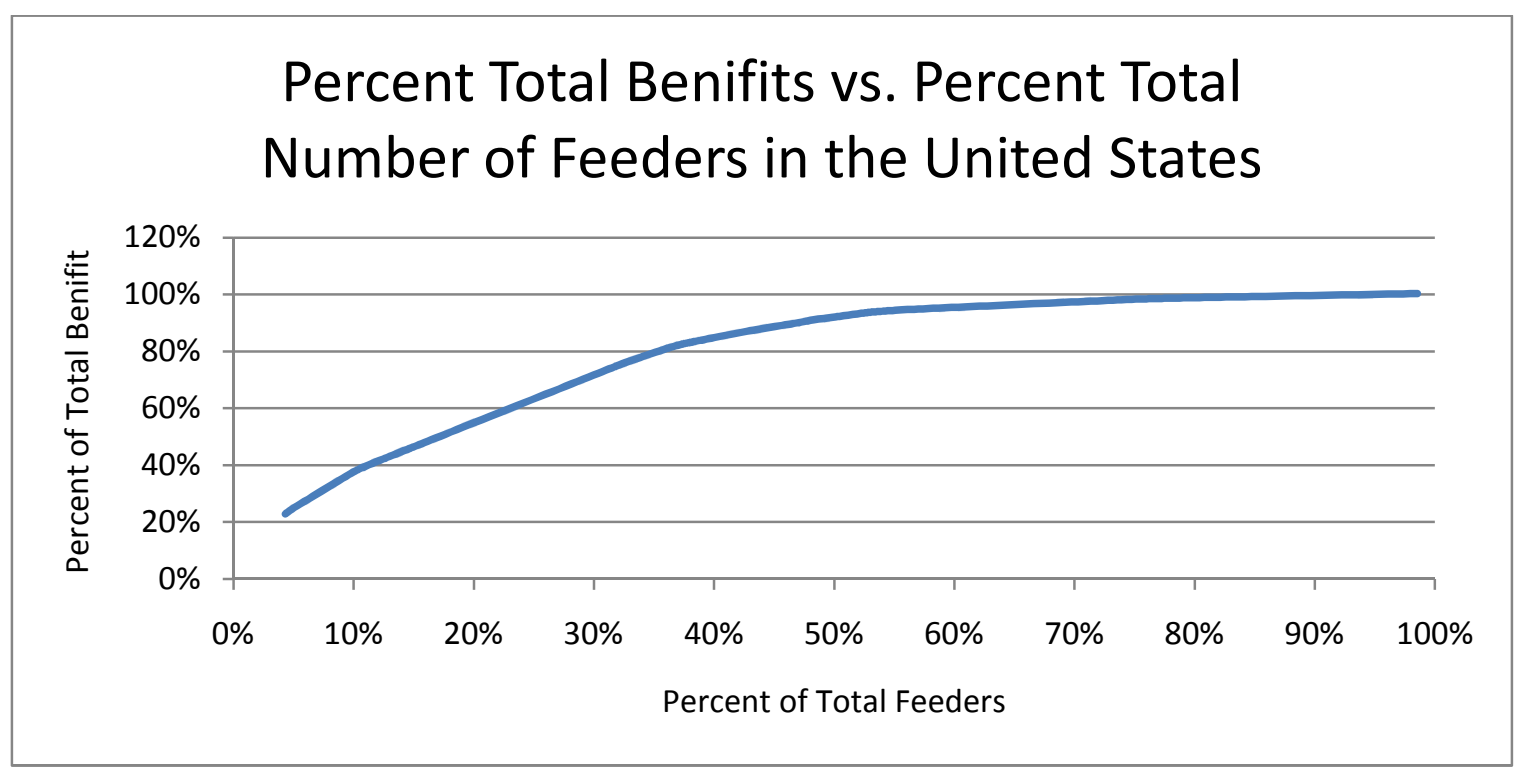

Figure 4.1: Percent Total Benefit vs. Percent Total Number of Feeders in the United States 


\section{Concluding Remarks}

This report has examined the benefits of deploying CVR and extrapolated those benefits to a national level. The CVR scheme implemented is 20 years old and is in the public domain, but newer proprietary methods are expected to provide improved results. The principle results obtained from this analysis are as follow:

1) The analysis of CVR, as well as other smart grid technologies, requires the use of time-series simulations.

2) The behavior of end use loads is more complicated than generally acknowledged. Voltage dependent multi-state models must be used.

3) CVR provides peak load reduction and annual energy reduction of approximately $0.5 \%-3 \%$ depending on the specific feeder.

4) When extrapolated to a national level it can be seen that a complete deployment of CVR, $100 \%$ of distribution feeders, provides a $3.04 \%$ reduction in annual energy consumption.

5) If deployed only on high value distribution feeders, $40 \%$ of distribution feeders, the annual energy consumption is still reduced by $2.4 \%$.

6) In a practical deployment of CVR heavily loaded higher voltage feeders should be targeted.

7) Loss reduction is not a significant benefit of CVR. 


\section{Appendix I: Regional CVR Plots}

Because of the large number of plots which are generated by the analysis of the 24 prototypical feeders, they have been collected into a single appendix.

\subsection{Region 1: CVR Plots}

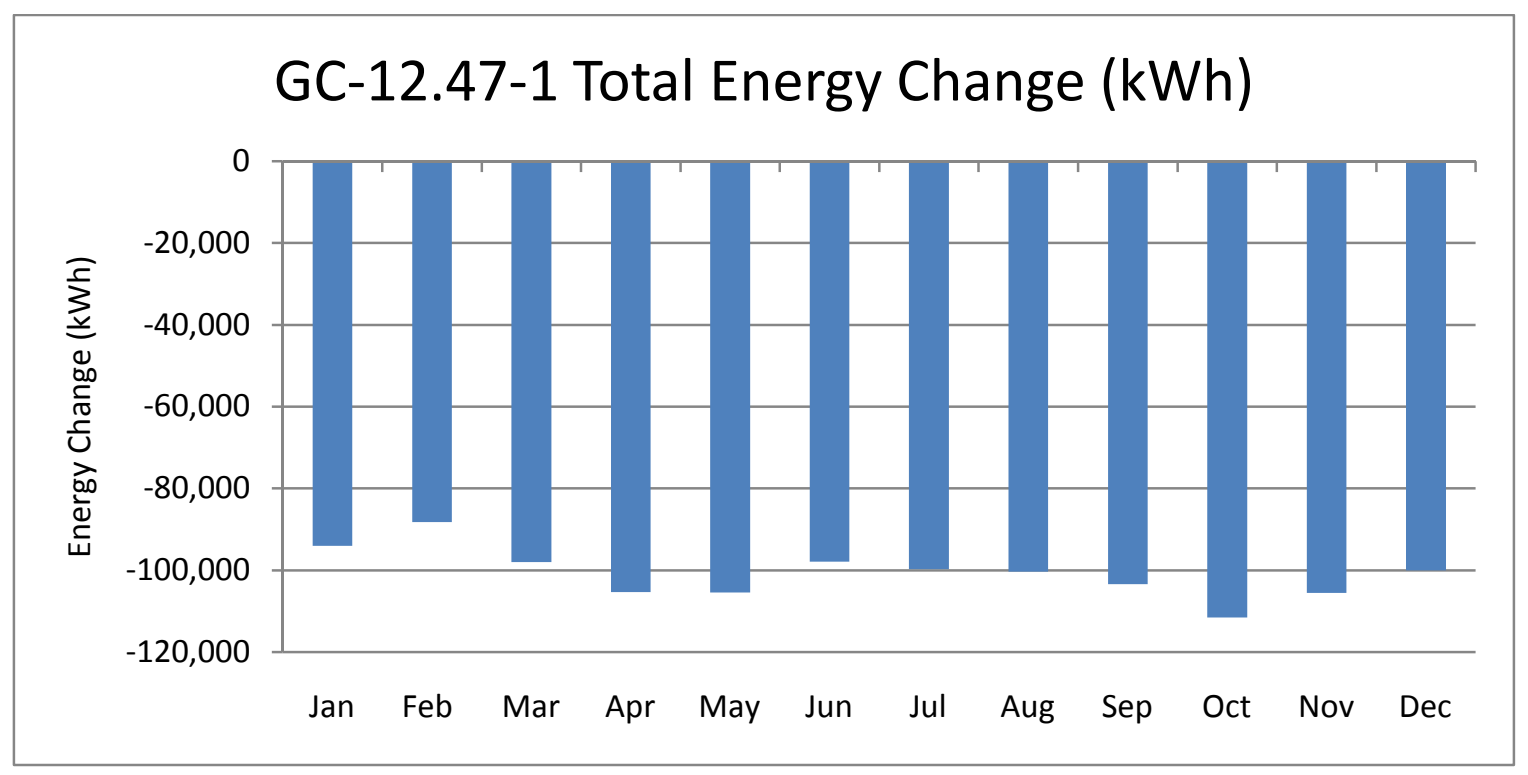

Figure 6.1: GC-12.47-1 Total Energy Change (kWh)

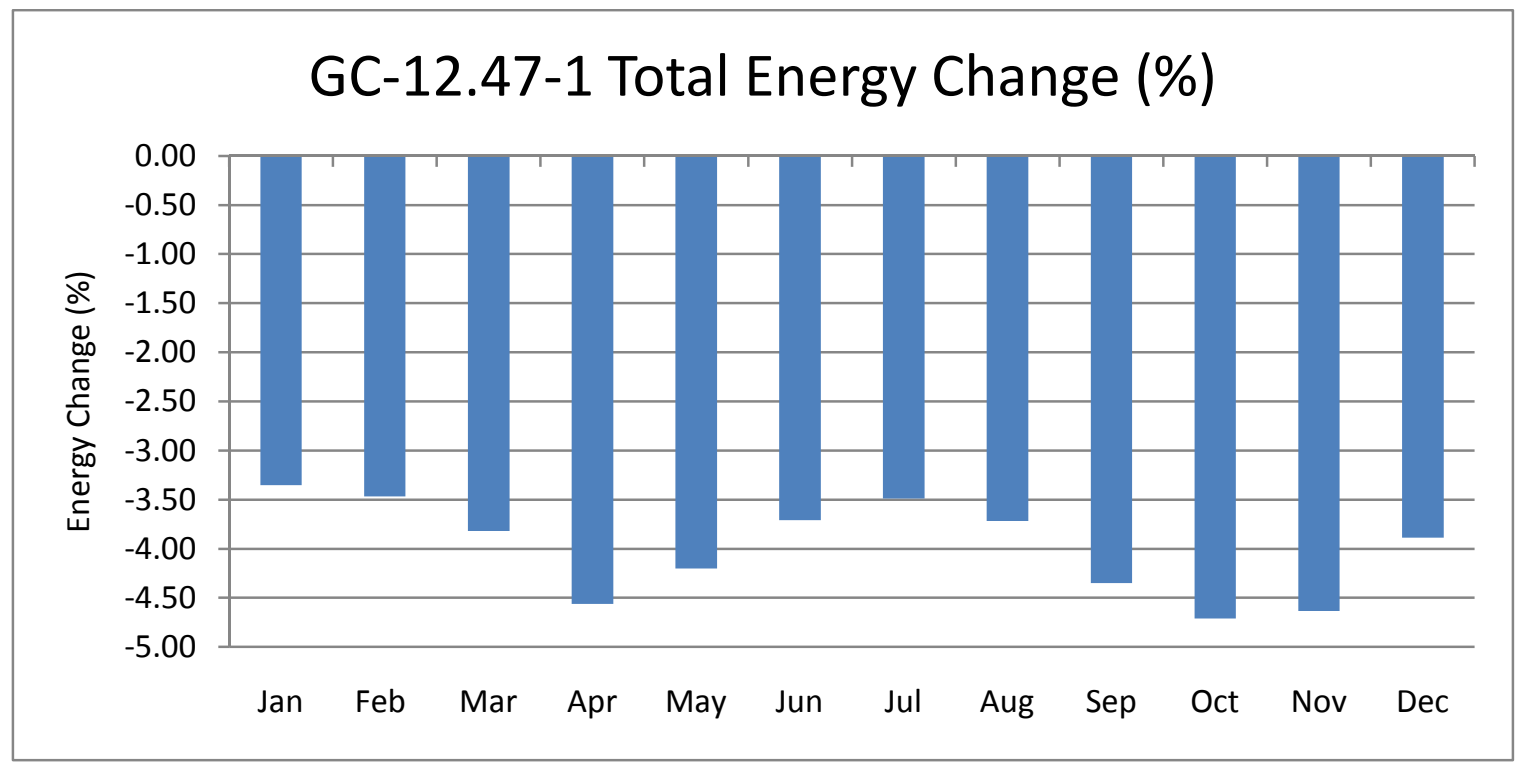

Figure 6.2: GC-12.47-1 Total Energy Change (\%) 


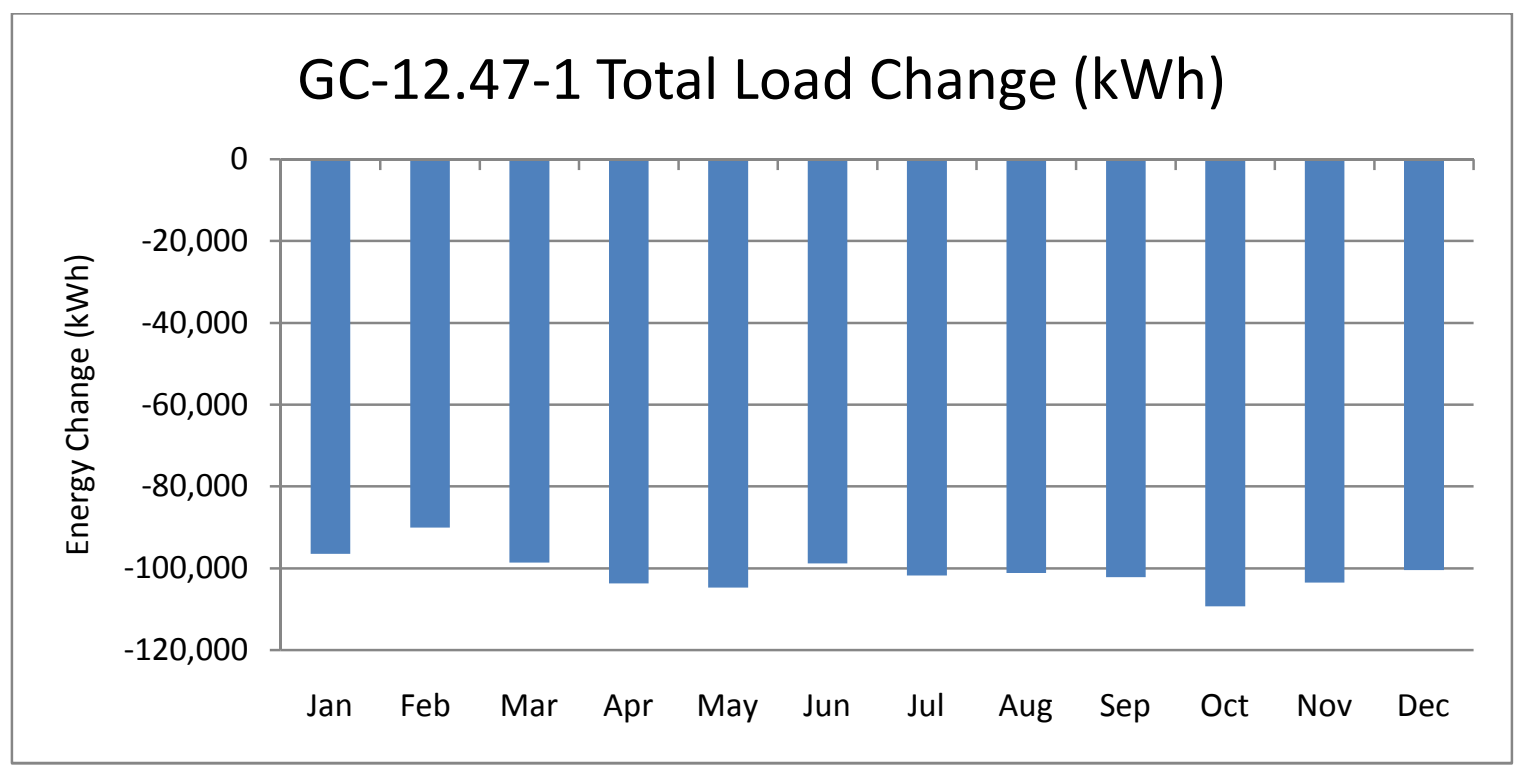

Figure 6.3: GC-12.47-1 Total Load Change (kWh)

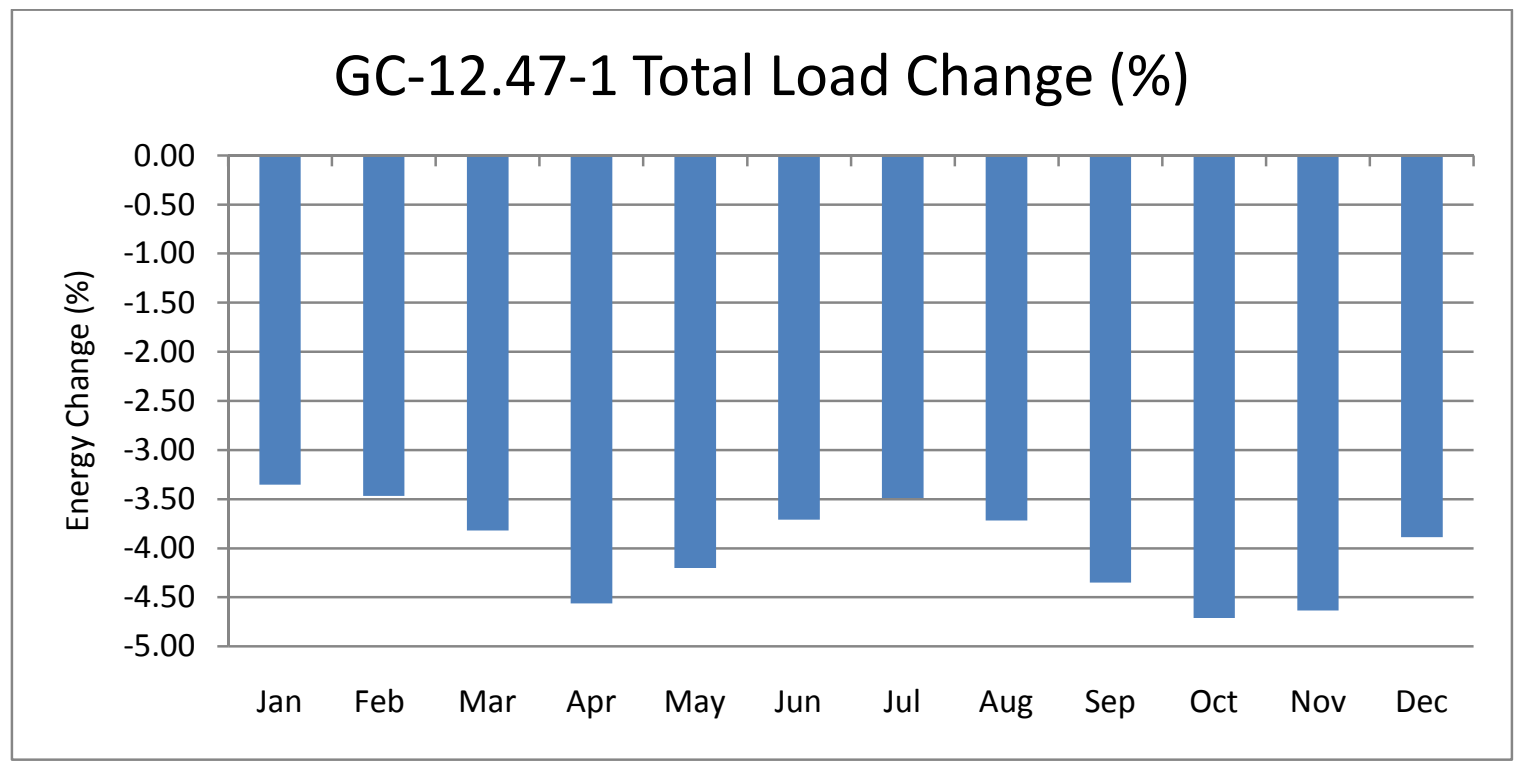

Figure 6.4: GC-12.47-1 Total Load Change (\%) 


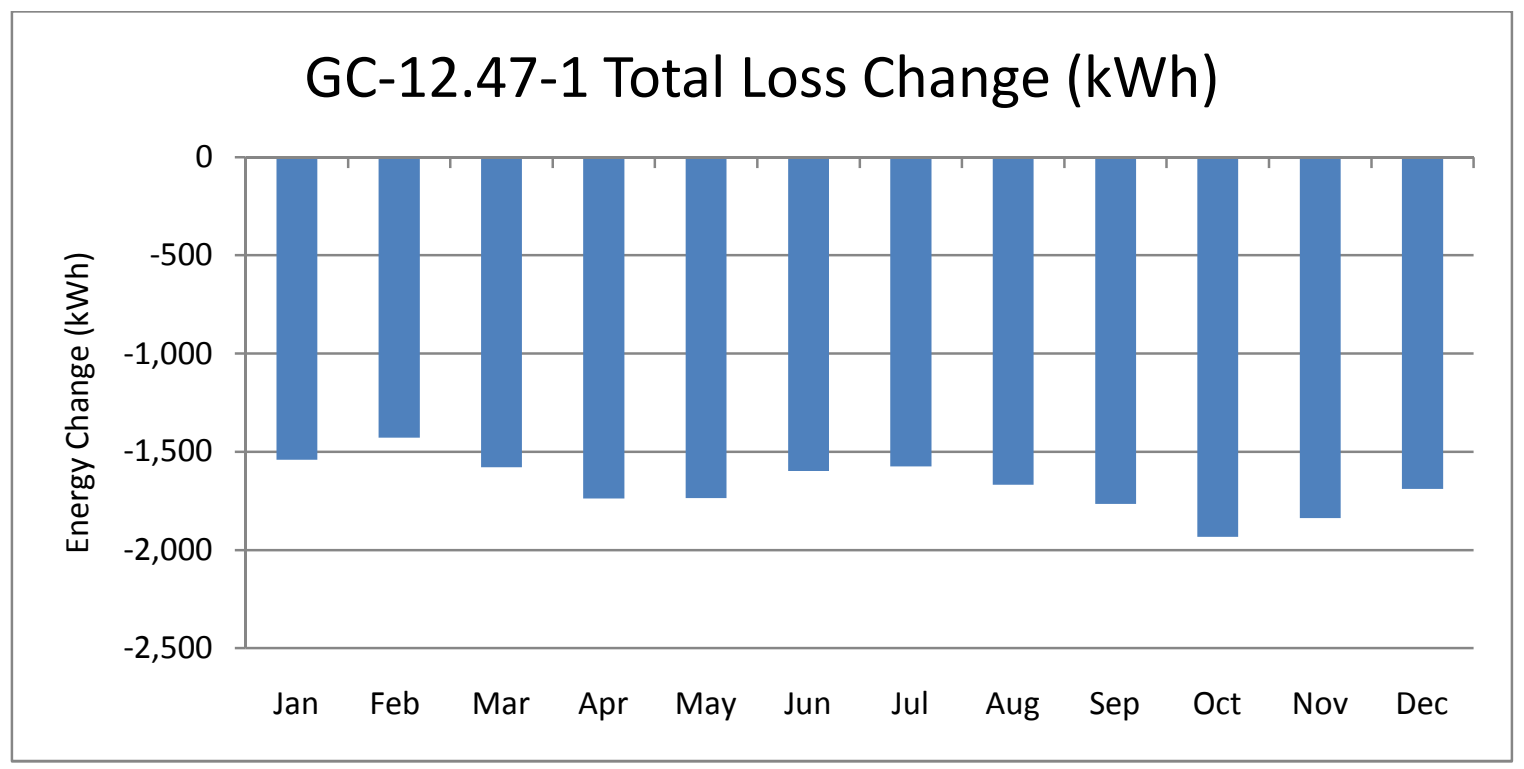

Figure 6.5: GC-12.47-1 Total Loss Change (kWh)

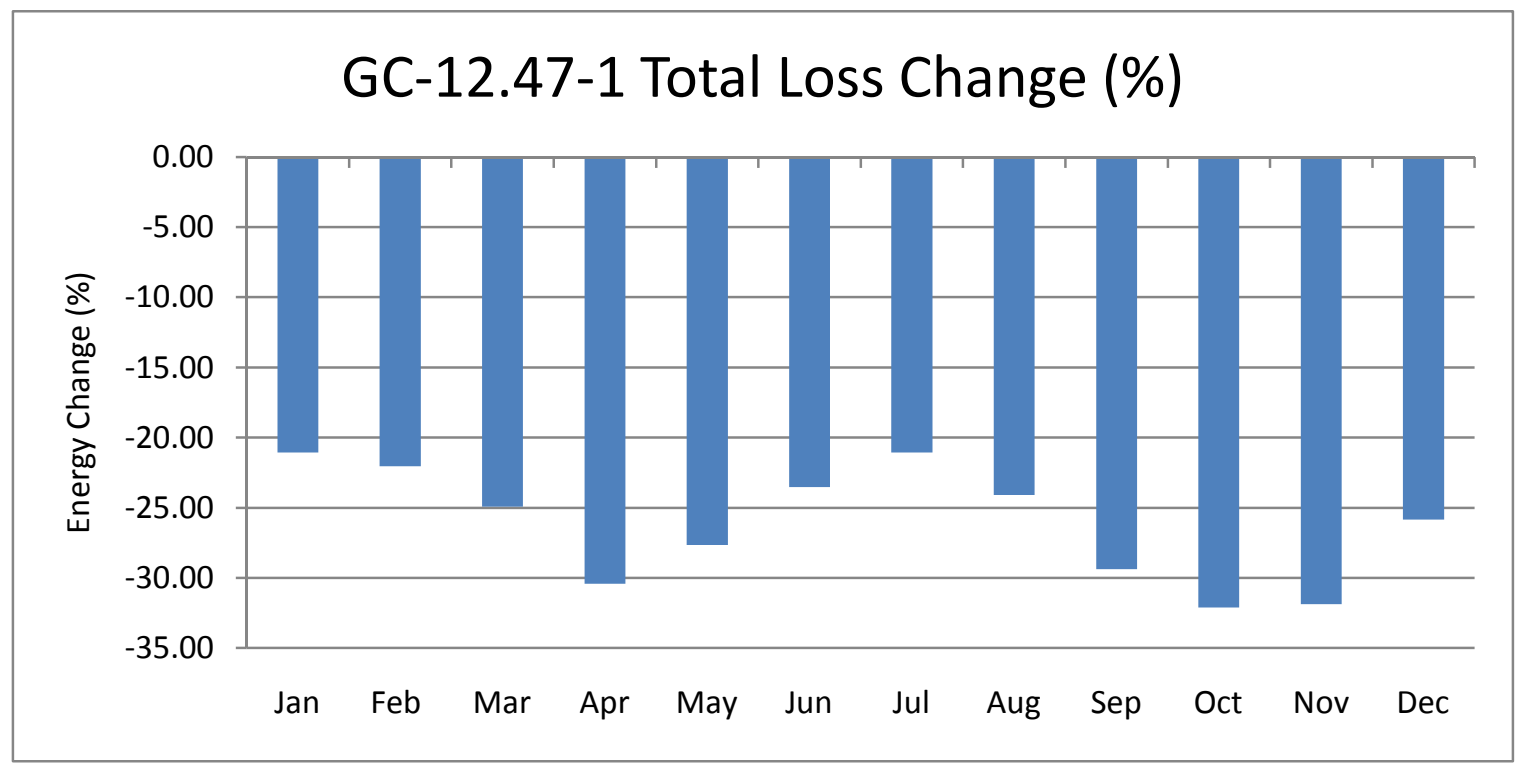

Figure 6.6: GC-12.47-1 Total loss Change (\%) 


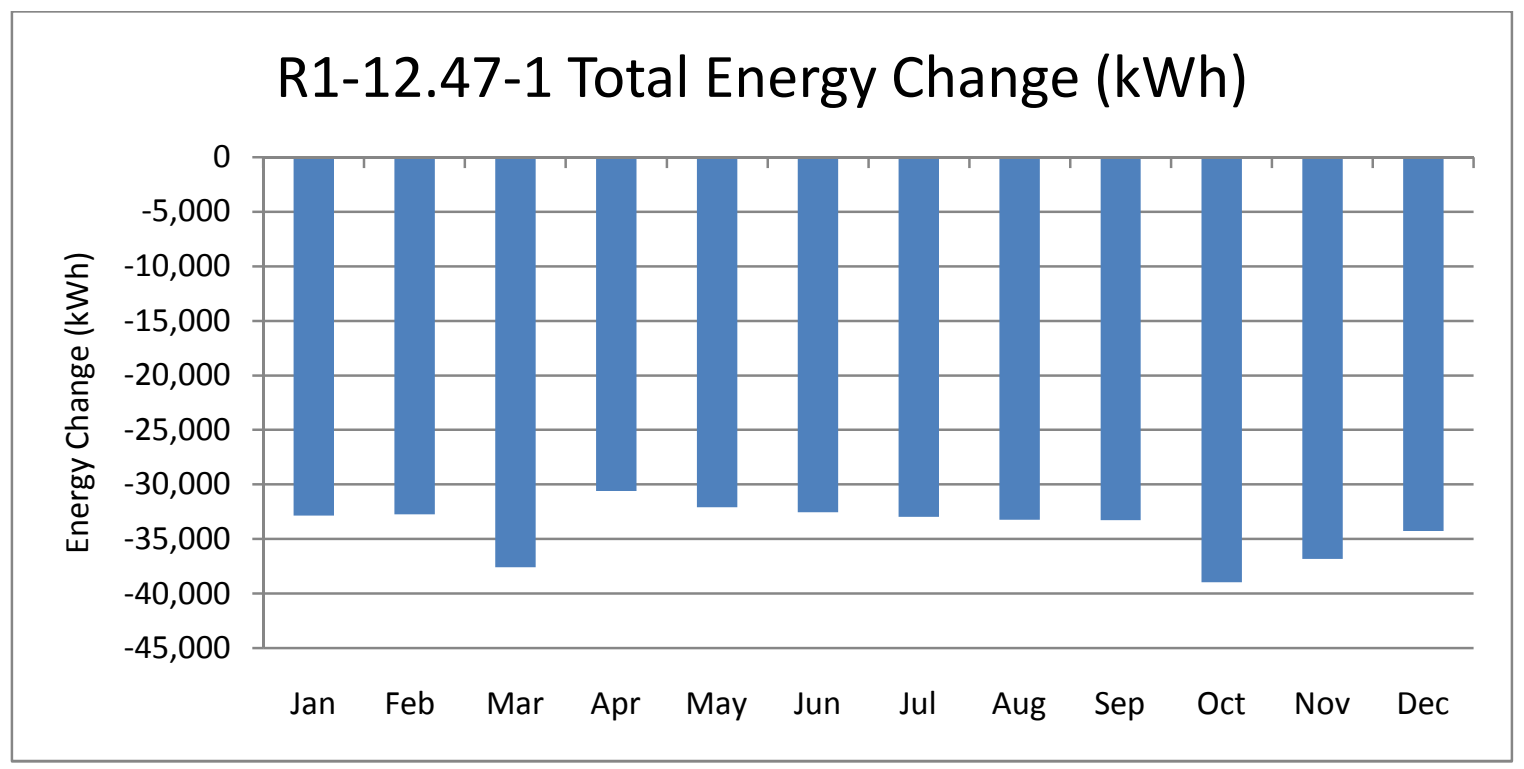

Figure 6.7: R1-12.47-1 Total Energy Change (kWh)

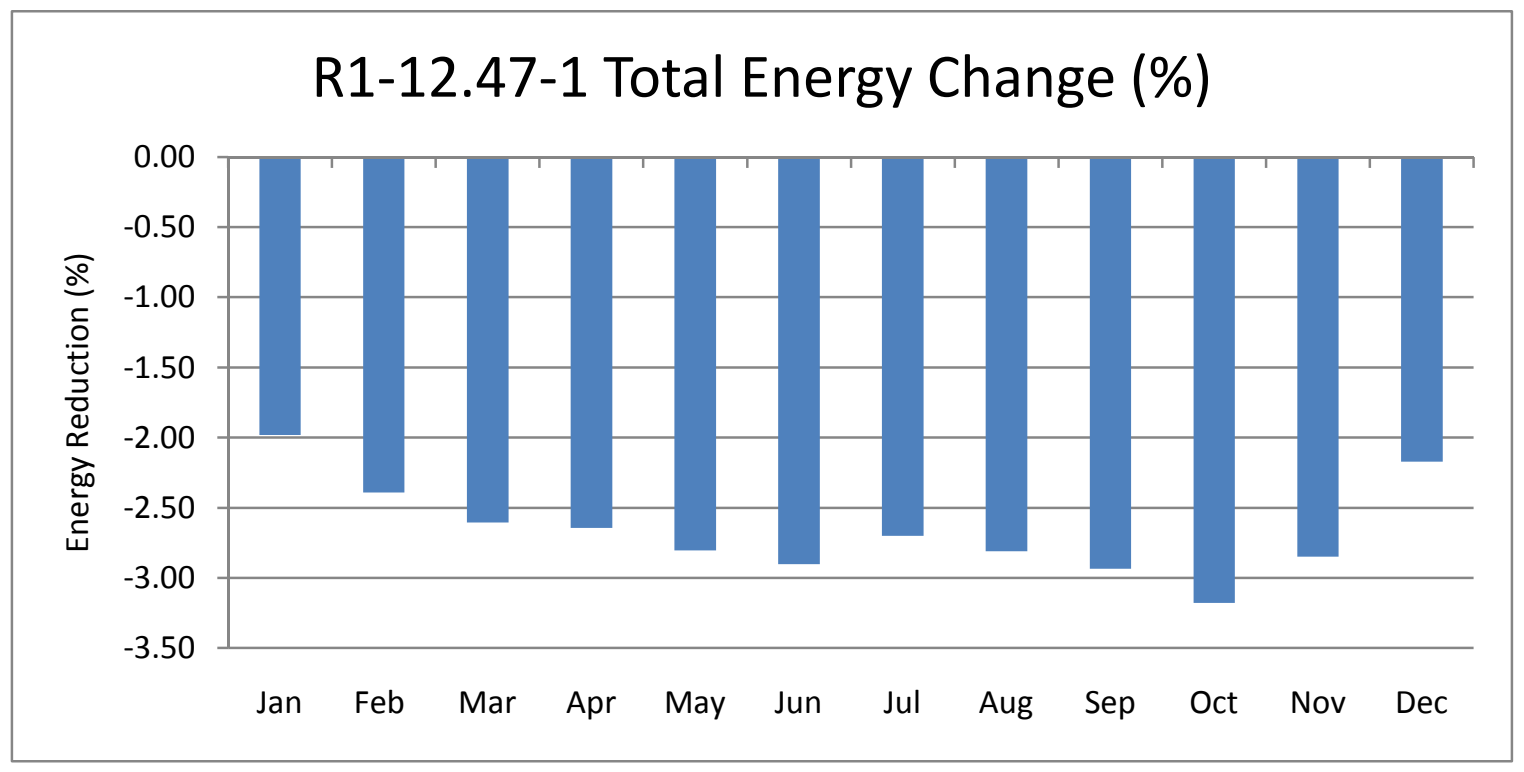

Figure 6.8: R1-12.47-1 Total Energy Change (\%) 


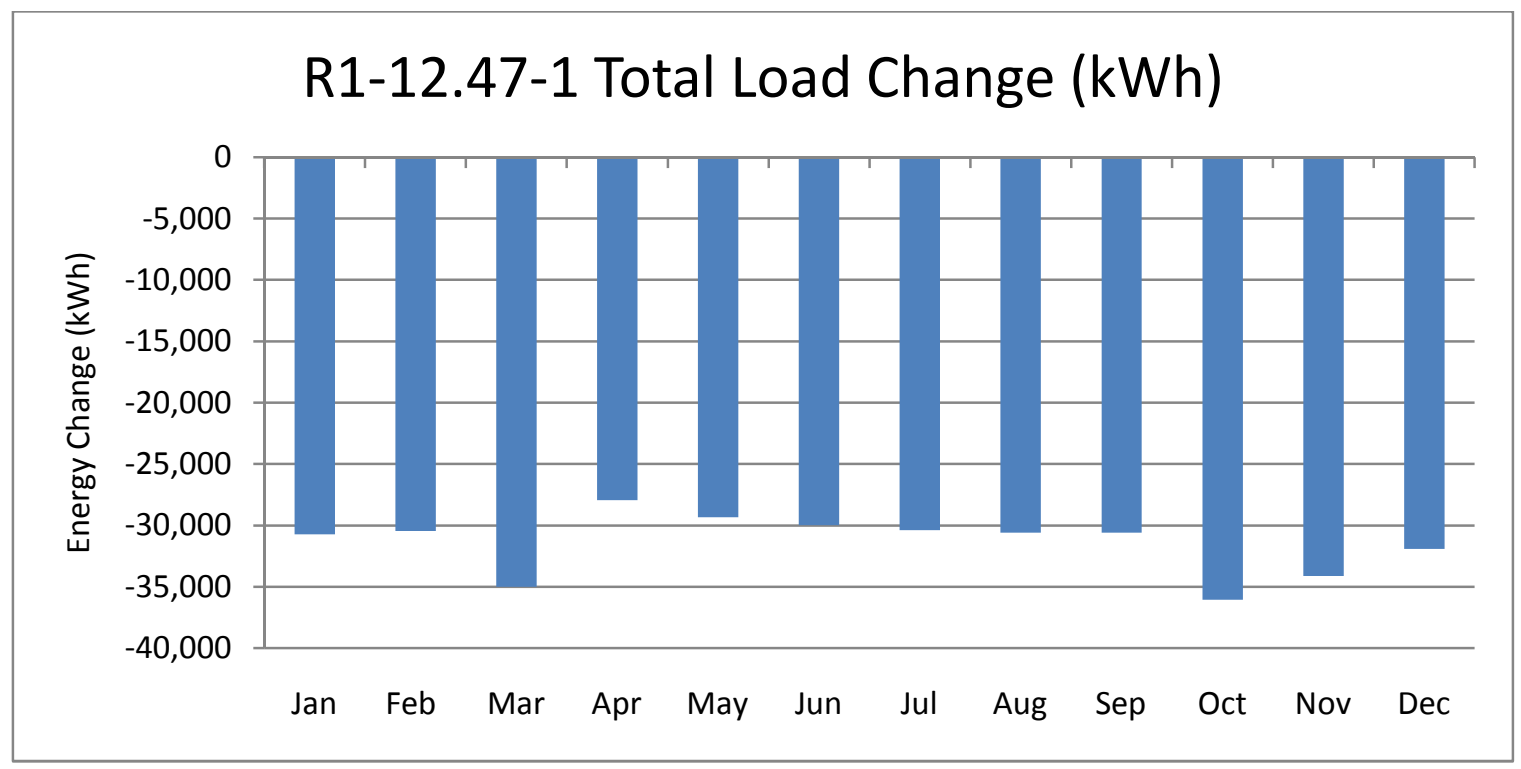

Figure 6.9: R1-12.47-1 Total Load Change (kWh)

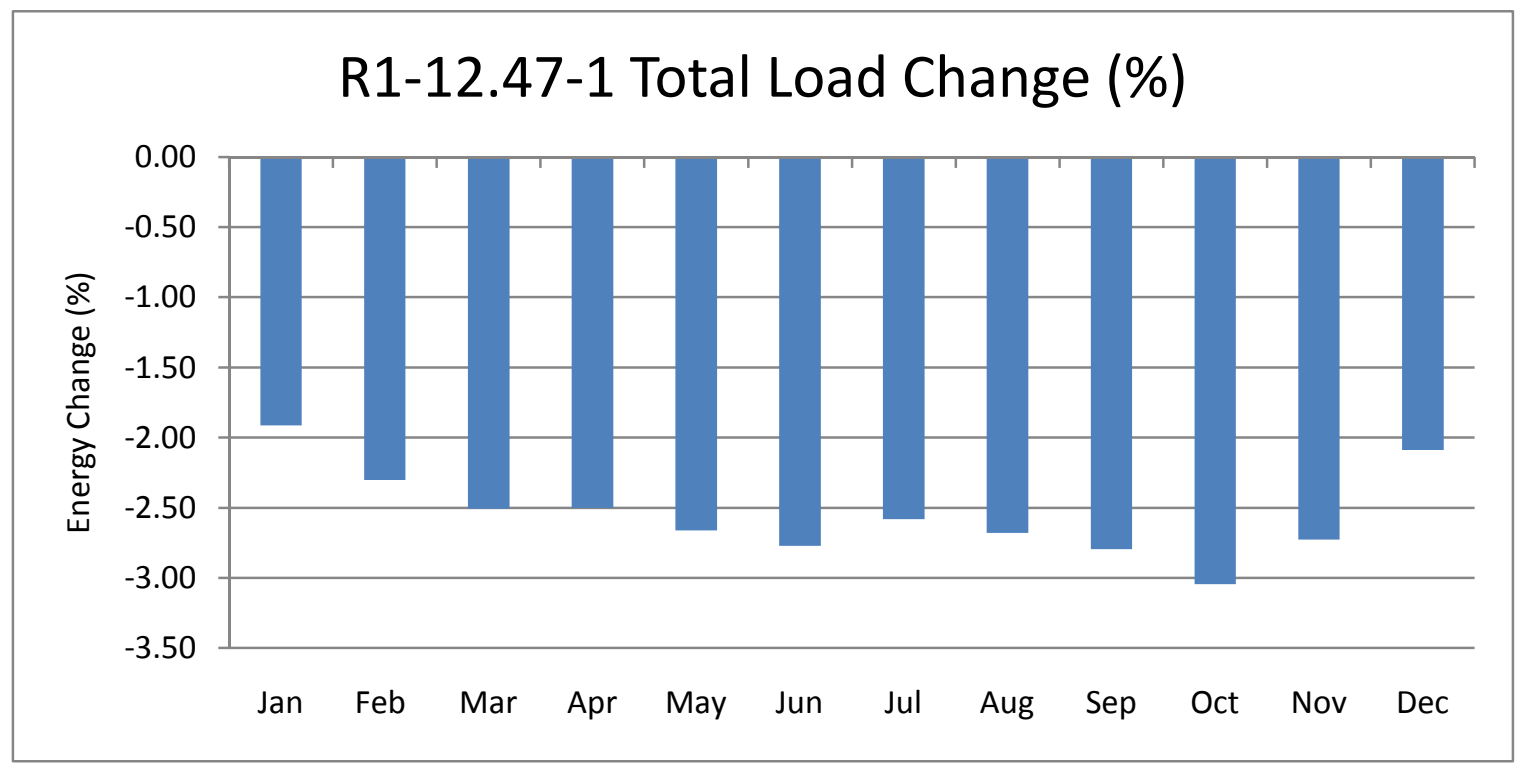

Figure 6.10: R1-12.47-1 Total Load Change (\%) 


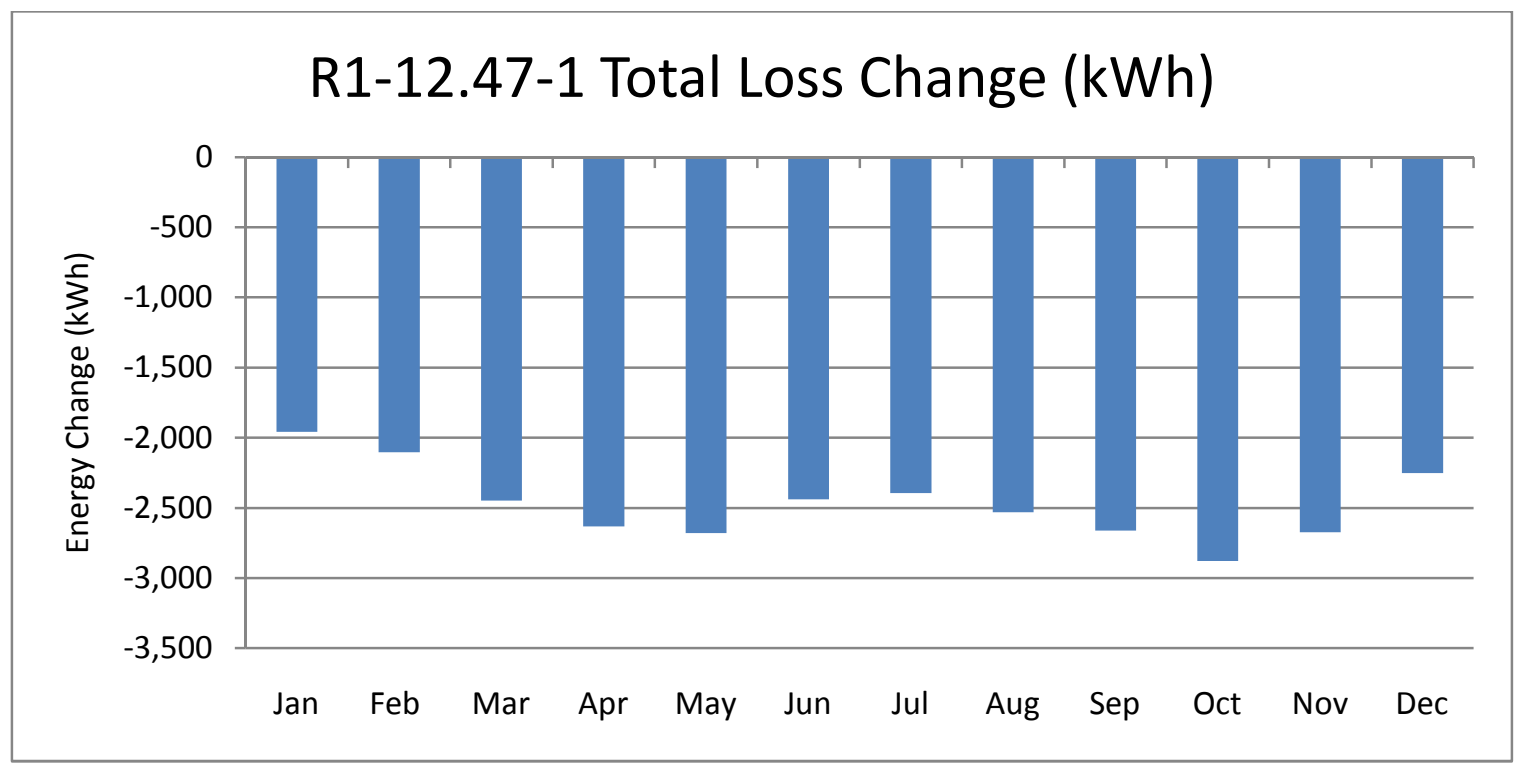

Figure 6.11: R1-12.47-1 Total Loss Change (kWh)

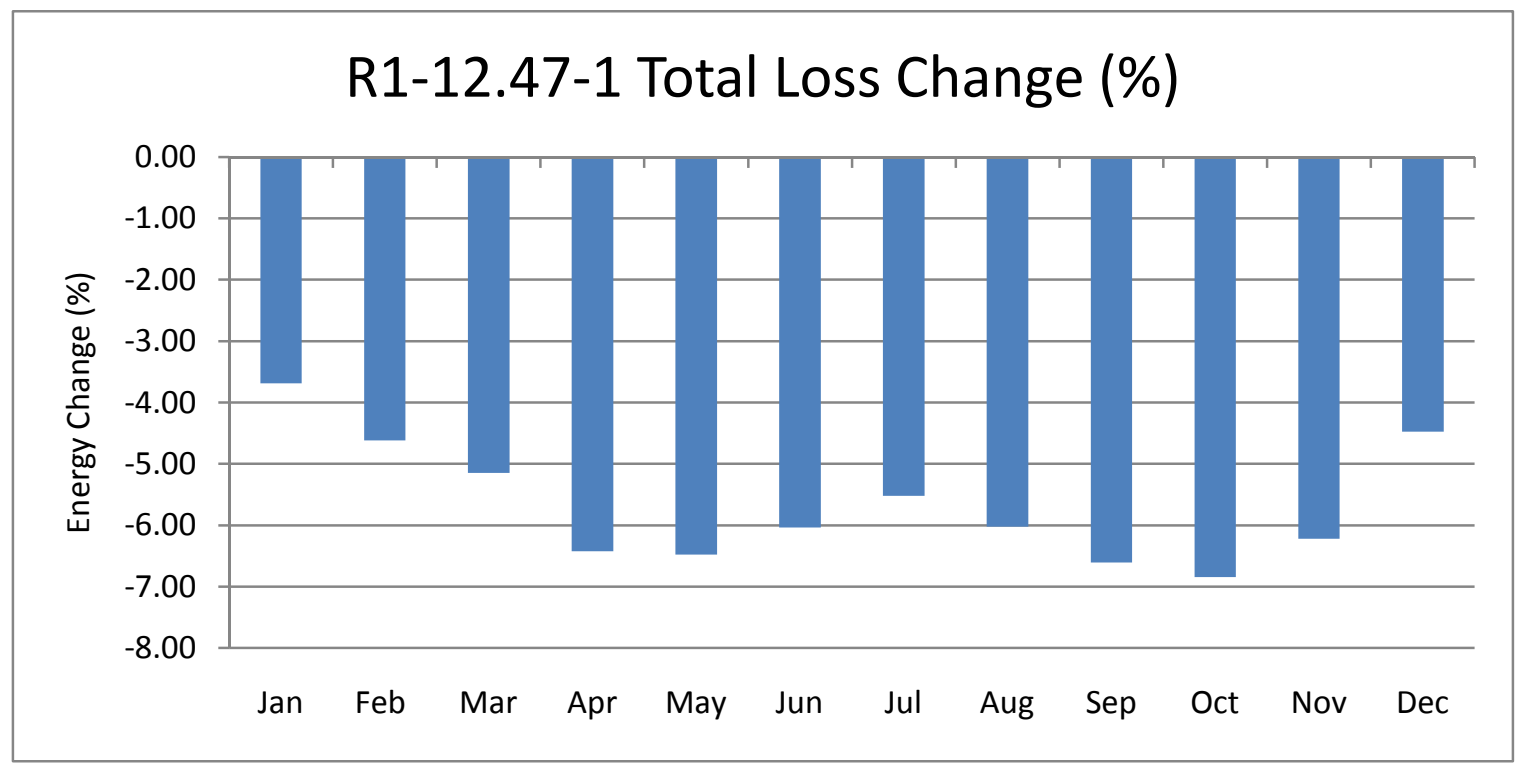

Figure 6.12: R1-12.47-1 Total Loss Change (\%) 


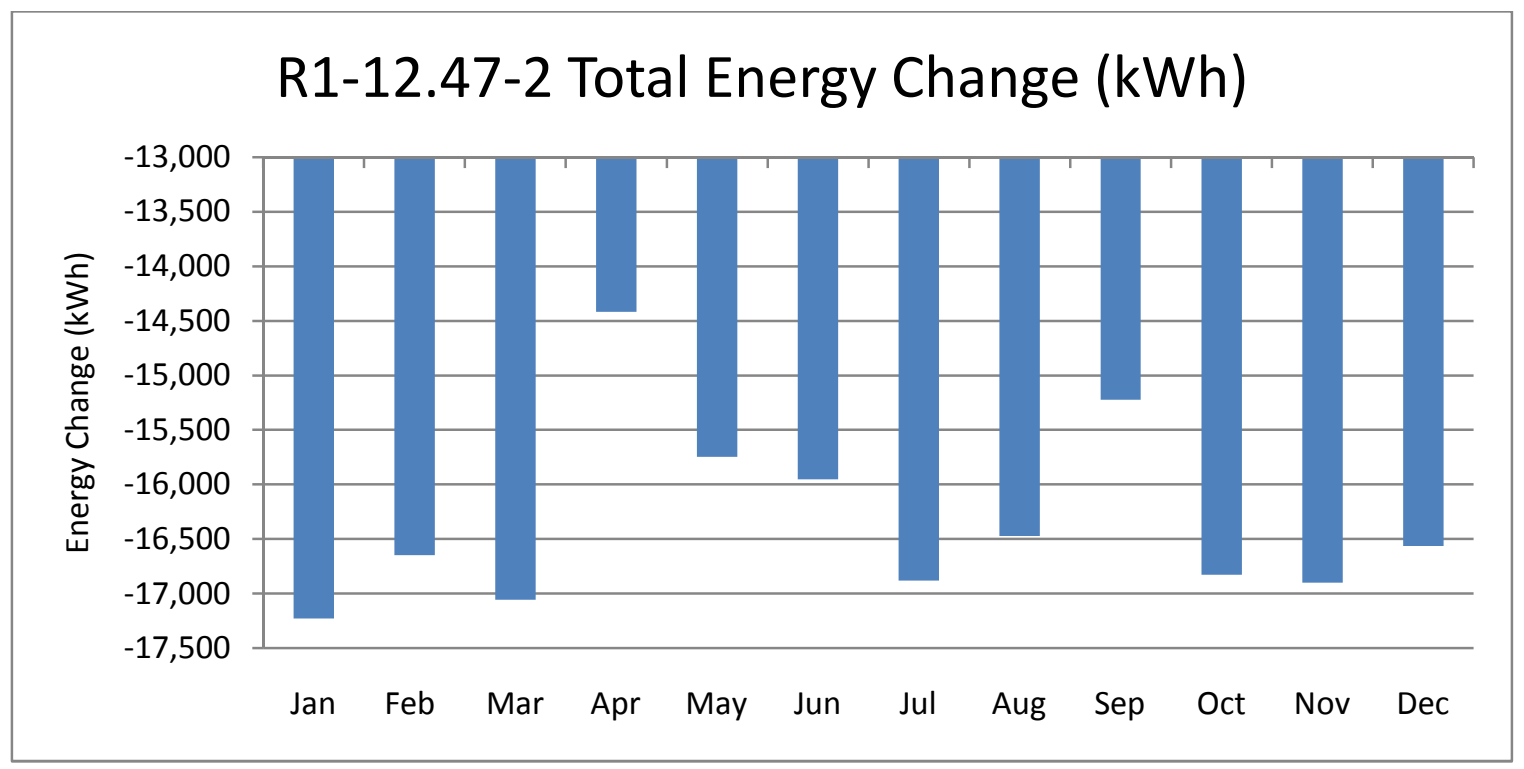

Figure 6.13: R1-12.47-2 Total Energy Change (kWh)

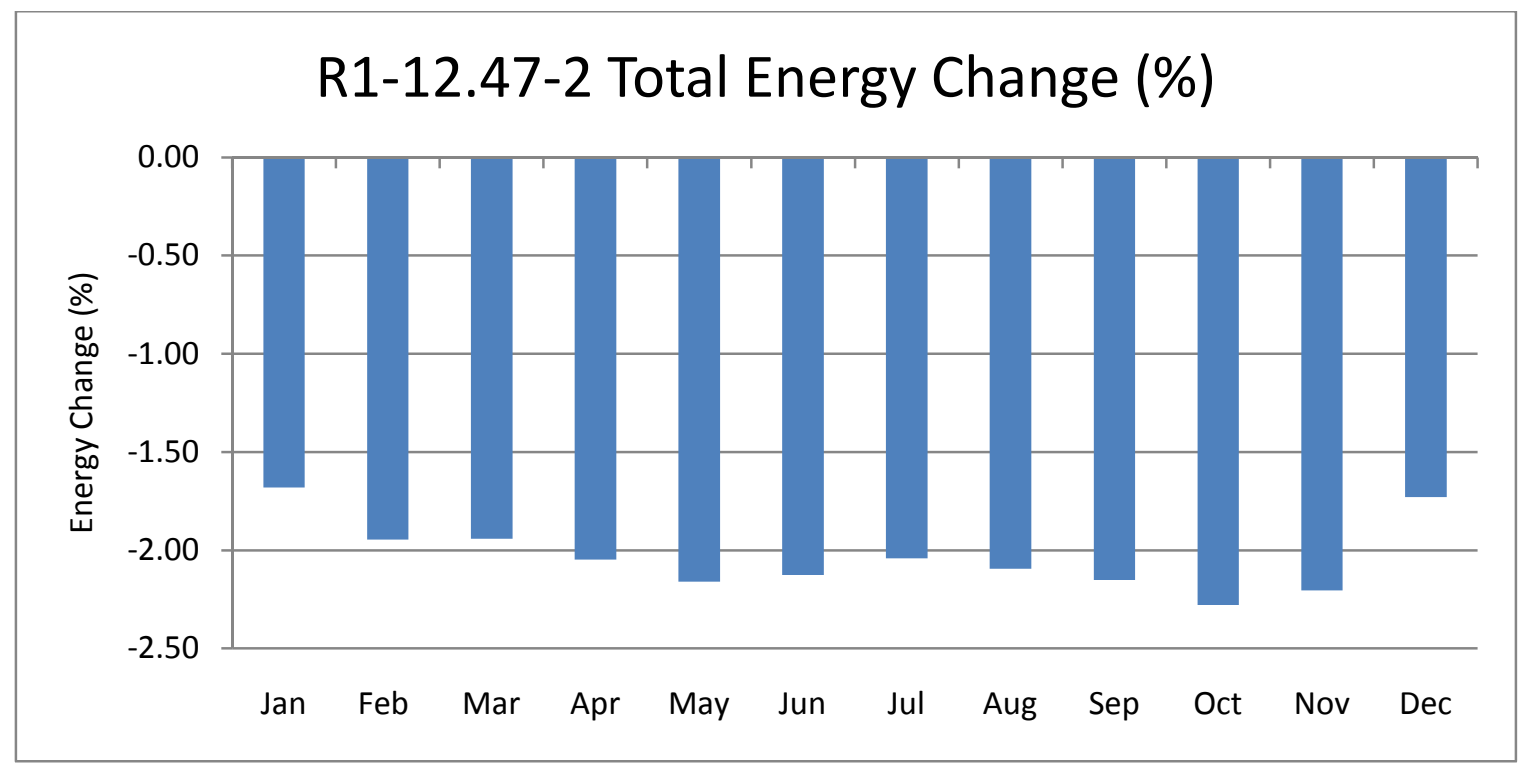

Figure 6.14: R1-12.47-2 Total Energy Change (\%) 


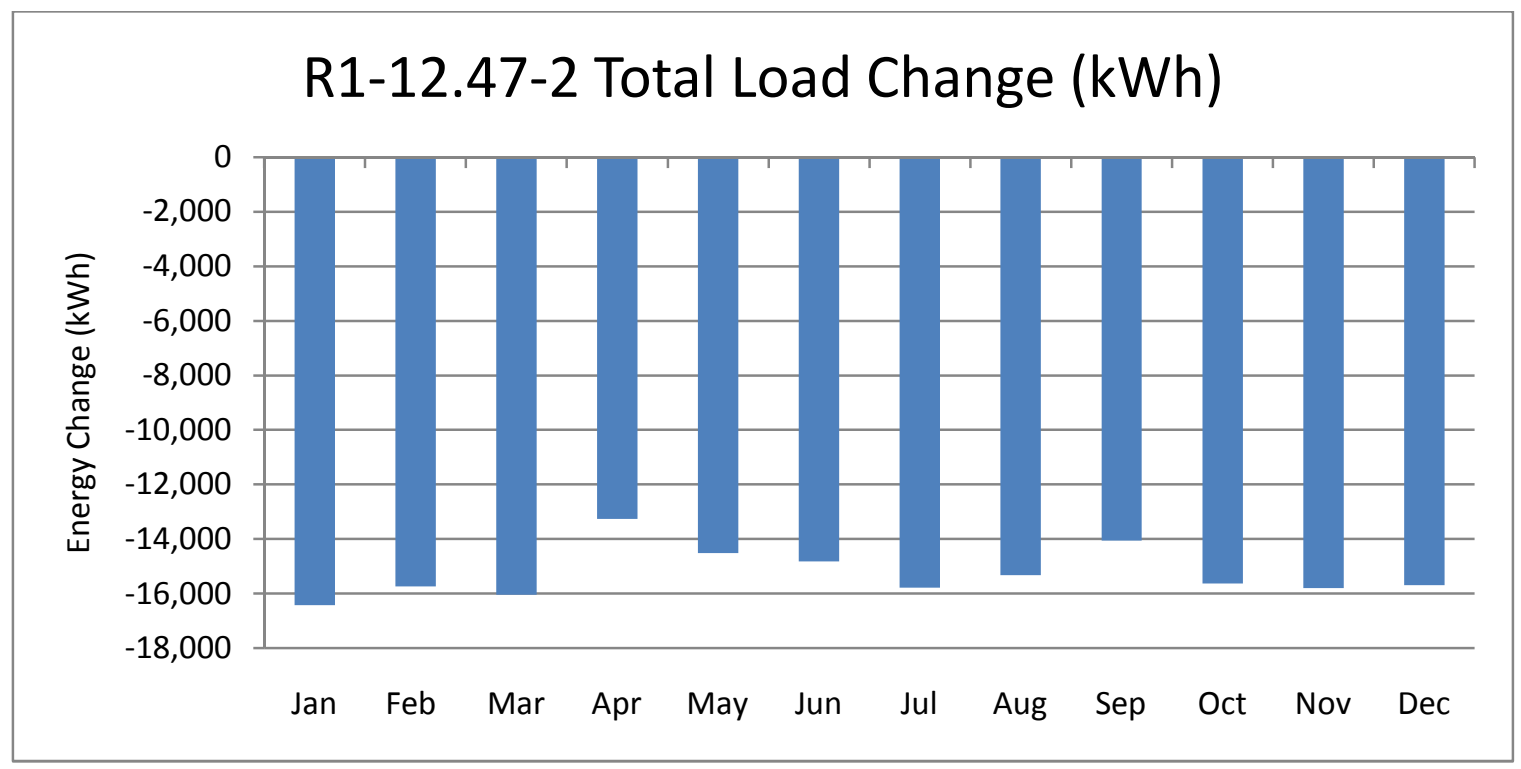

Figure 6.15: R1-12.47-2 Total Load Change (kWh)

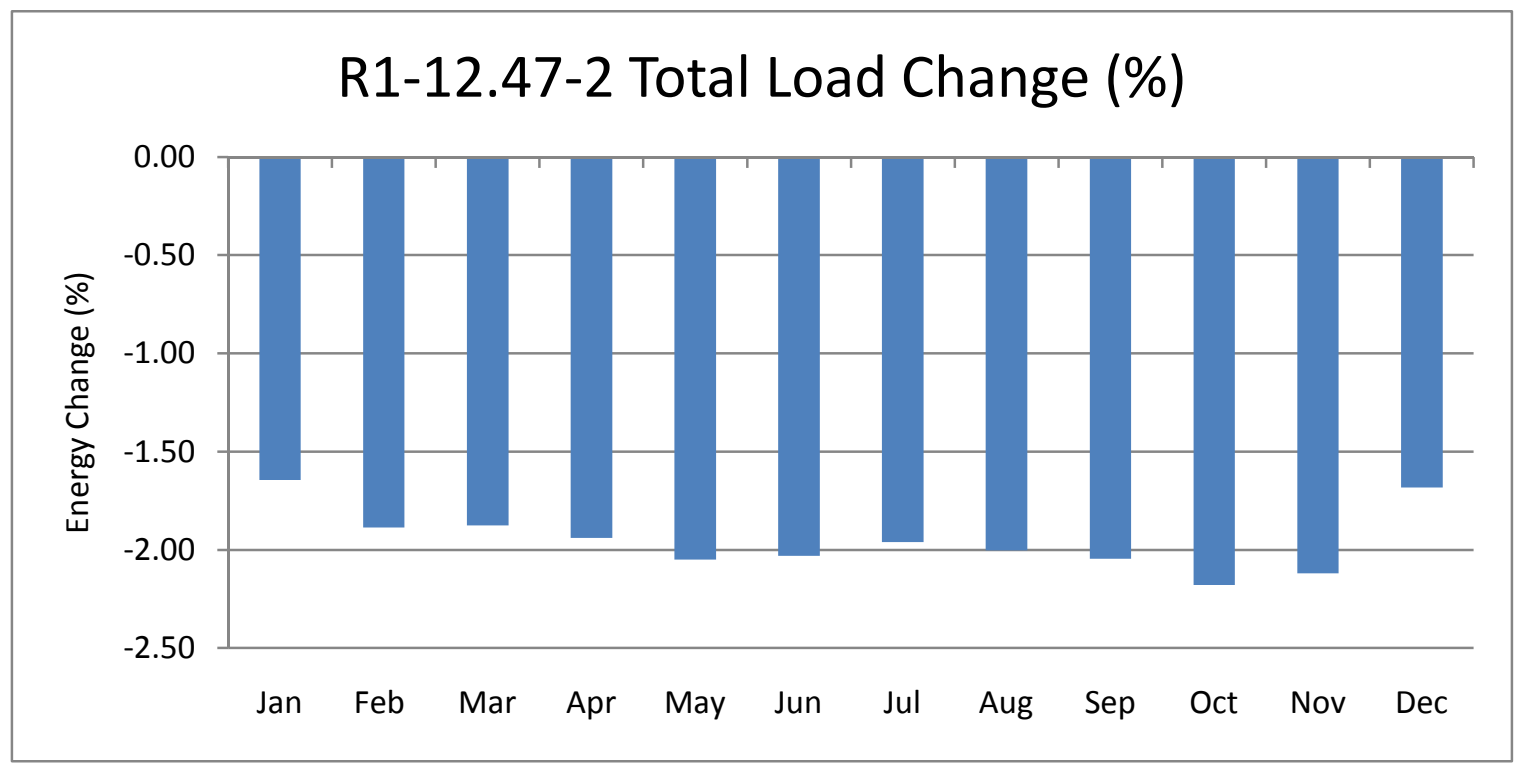

Figure 6.16: R1-12.47-2 Total Load Change (\%) 


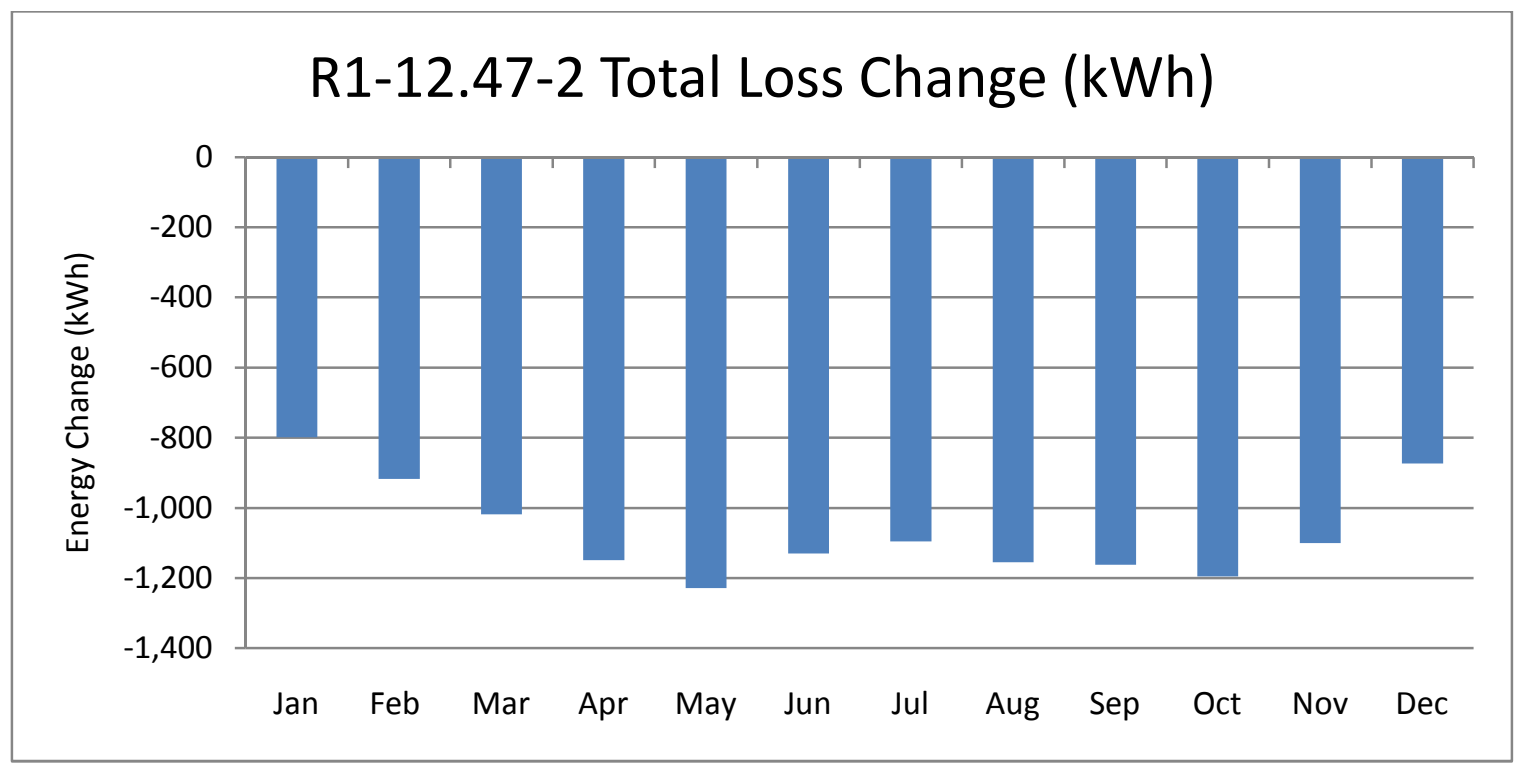

Figure 6.17: R1-12.47-2 Total Loss Change (kWh)

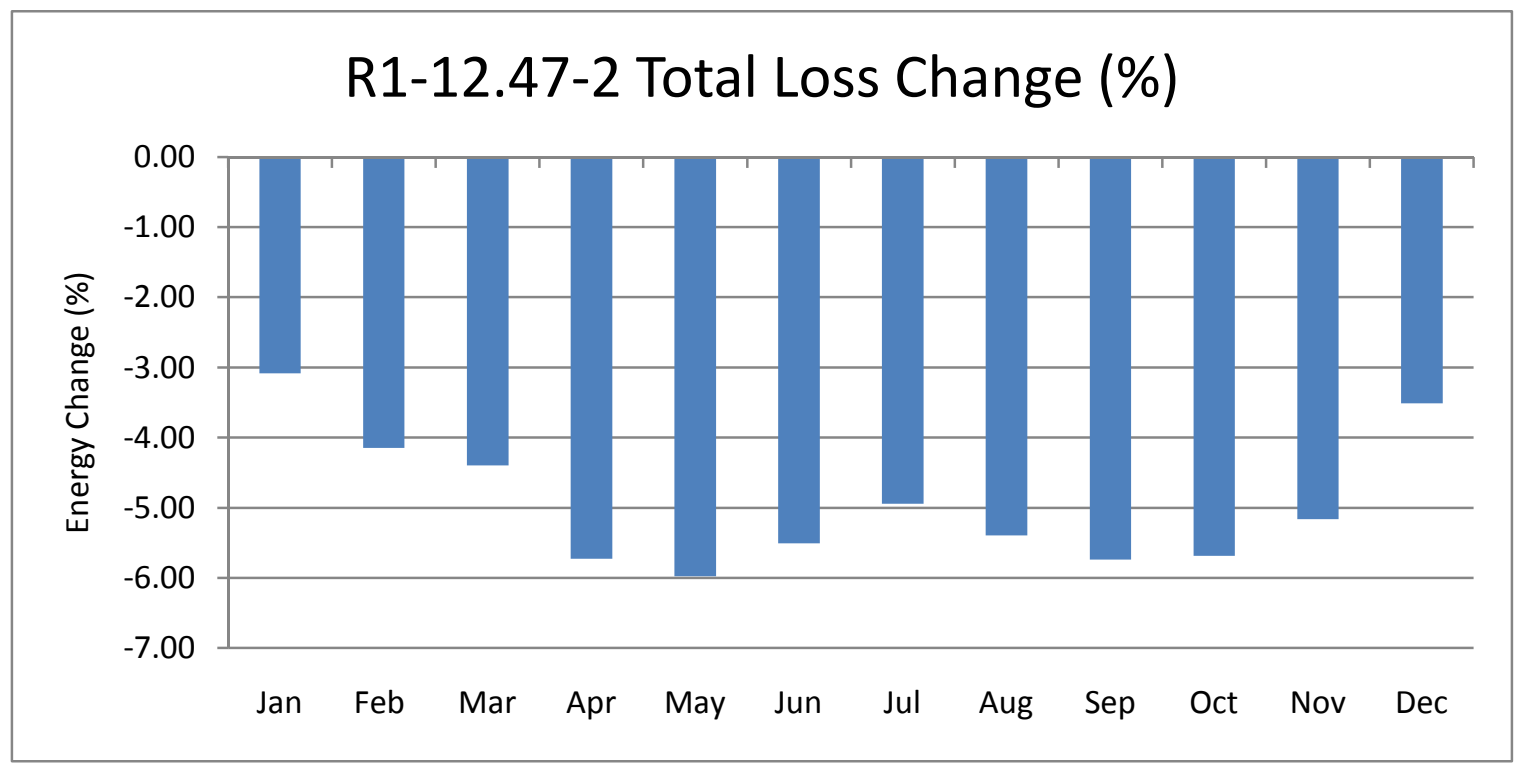

Figure 6.18: R1-12.47-2 Total Loss Change (\%) 


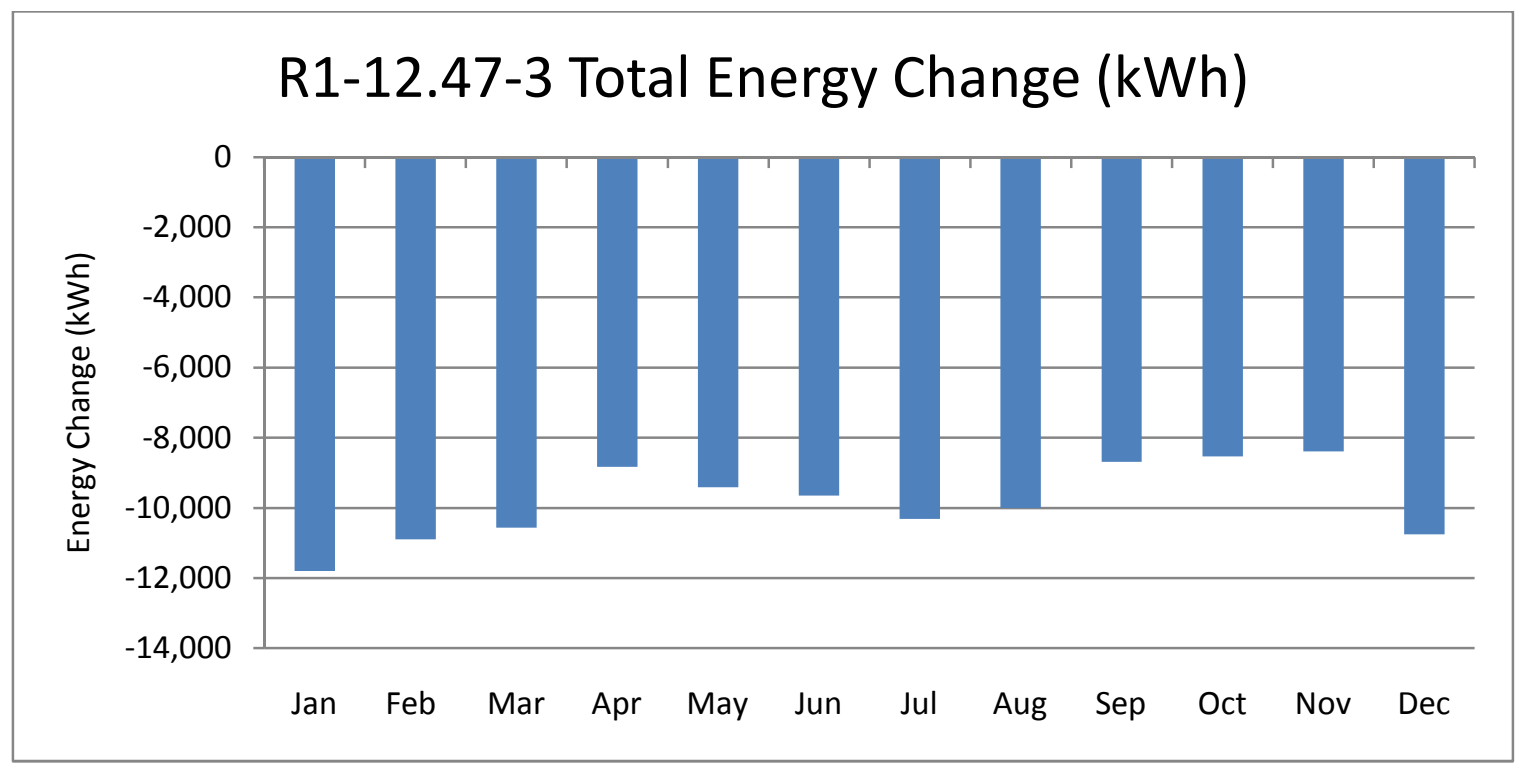

Figure 6.19: R1-12.47-3 Total Energy Change (kWh)

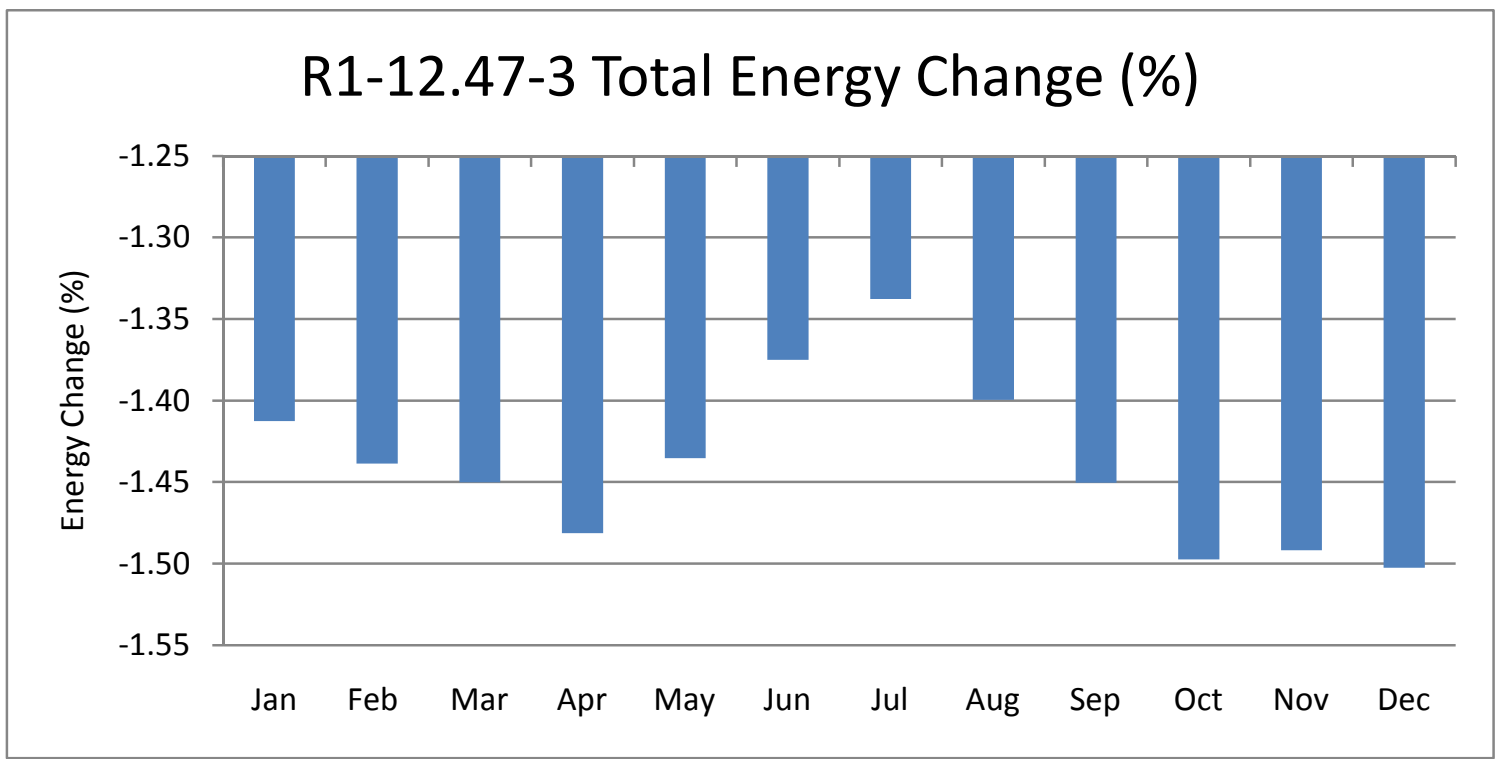

Figure 6.20: R1-12.47-3 Total Energy Change (\%) 


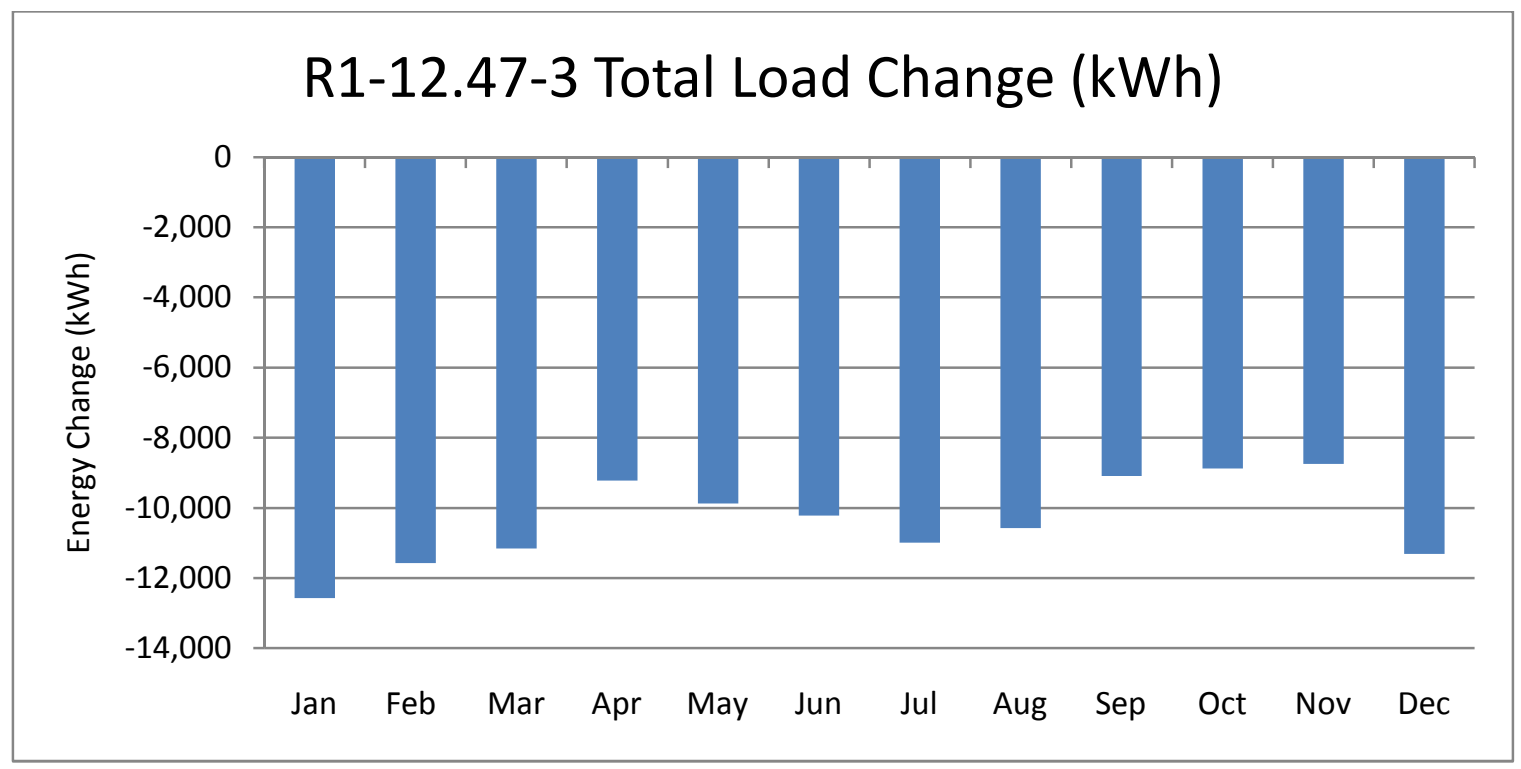

Figure 6.21: R1-12.47-3 Total Load Change (kWh)

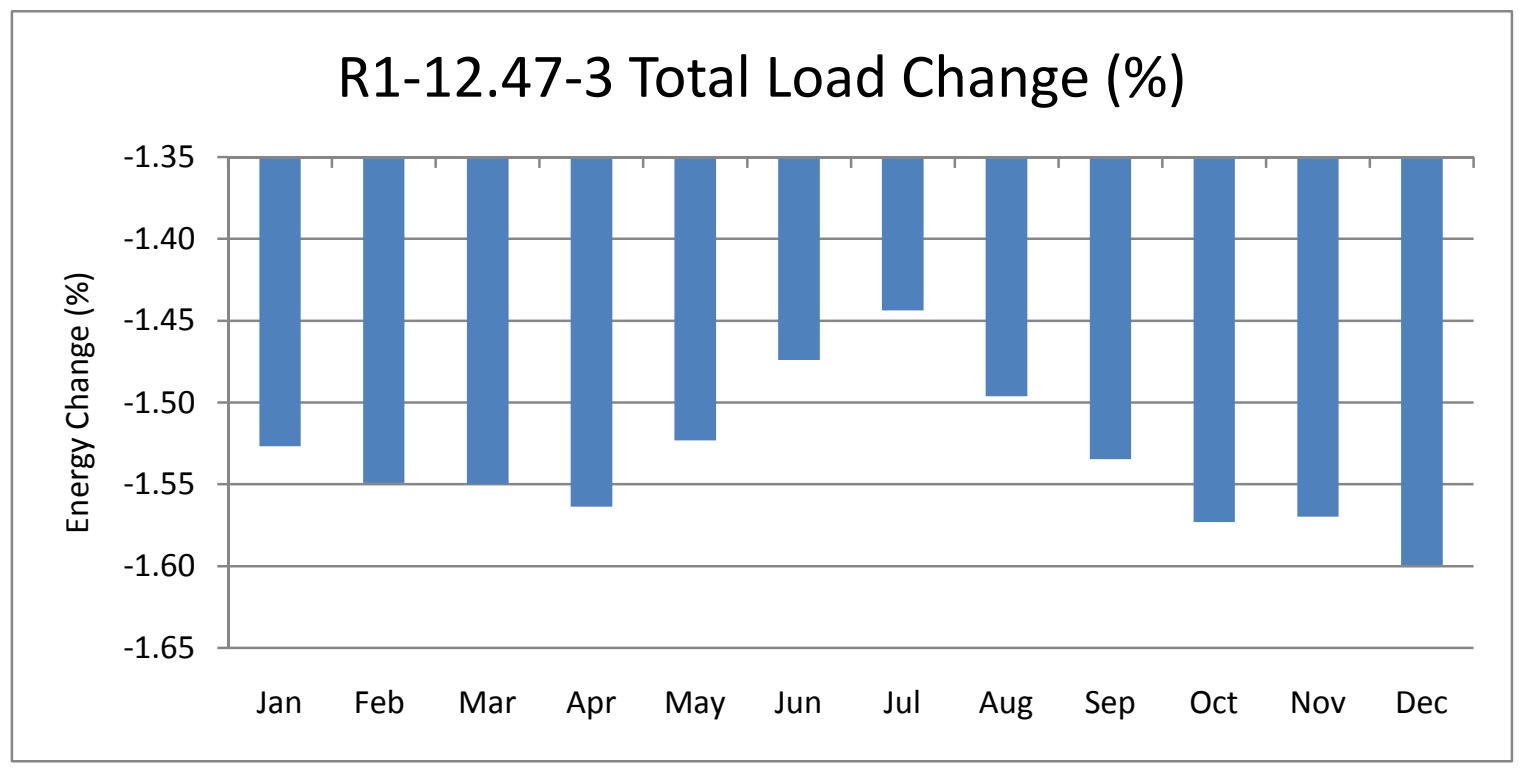

Figure 6.22: R1-12.47-3 Total Load Change (\%) 


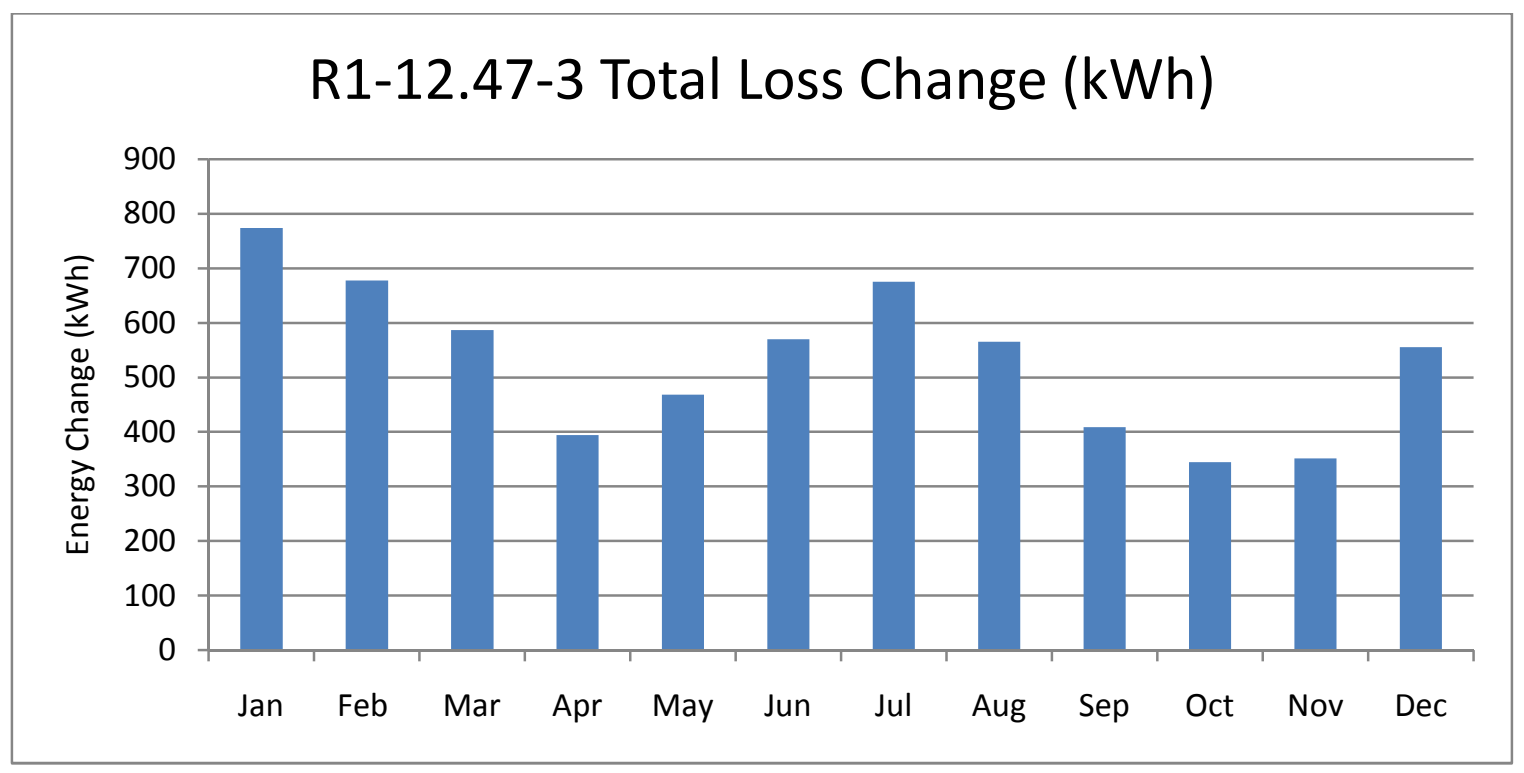

Figure 6.23: R1-12.47-3 Total Loss Change (kWh)

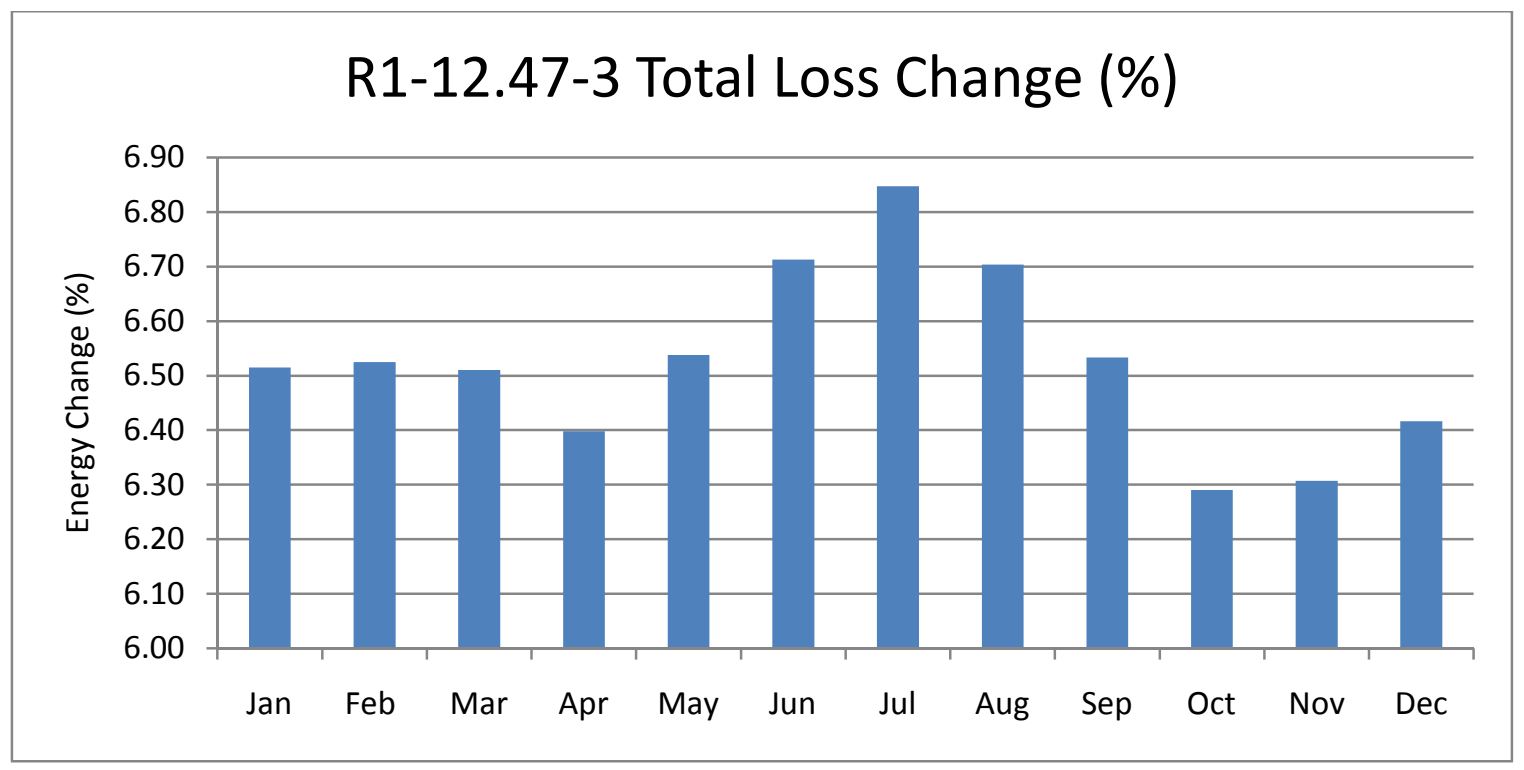

Figure 6.24: R1-12.47-3 Total Loss Change (\%) 


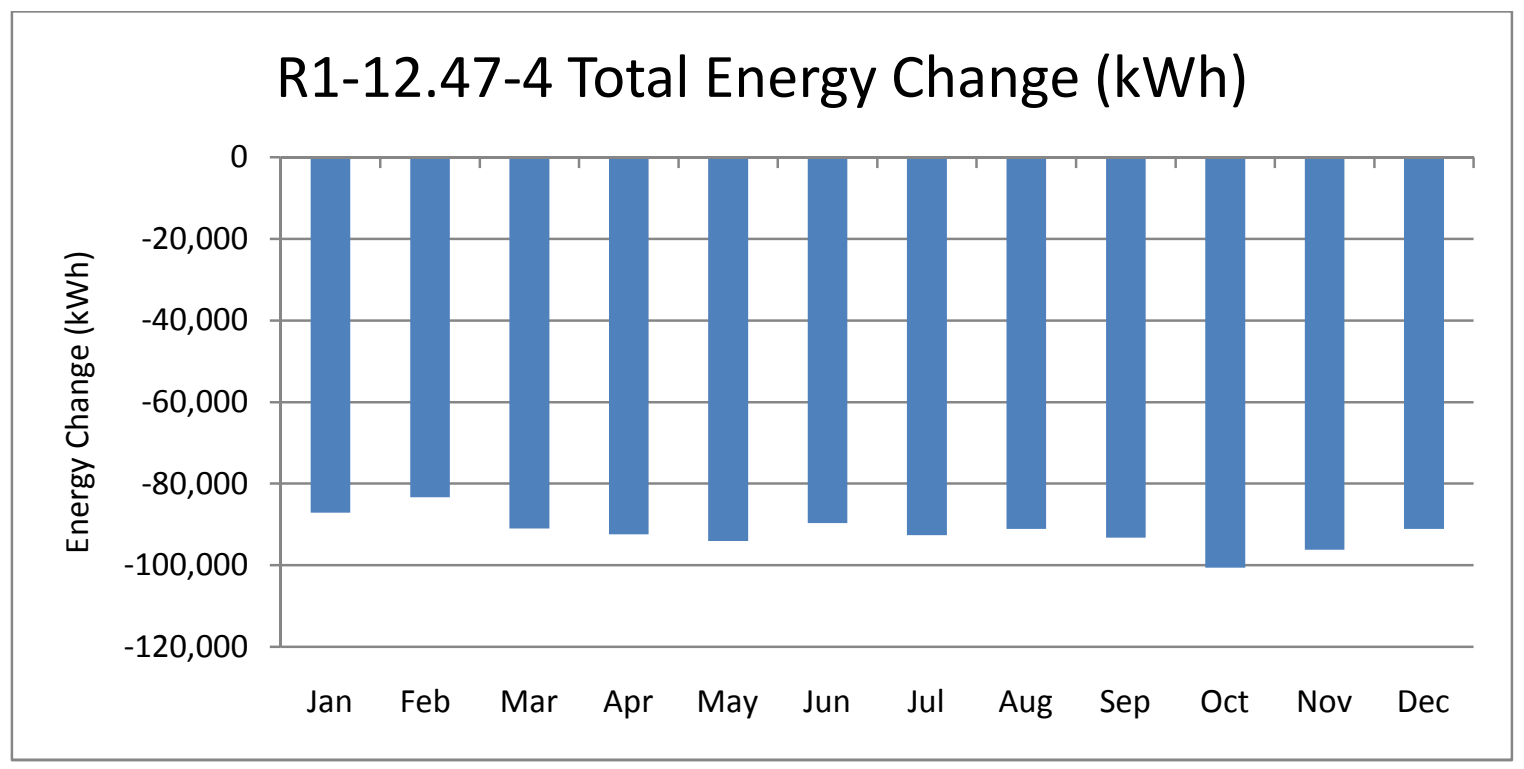

Figure 6.25: R1-12.47-4 Total Energy Change (kWh)

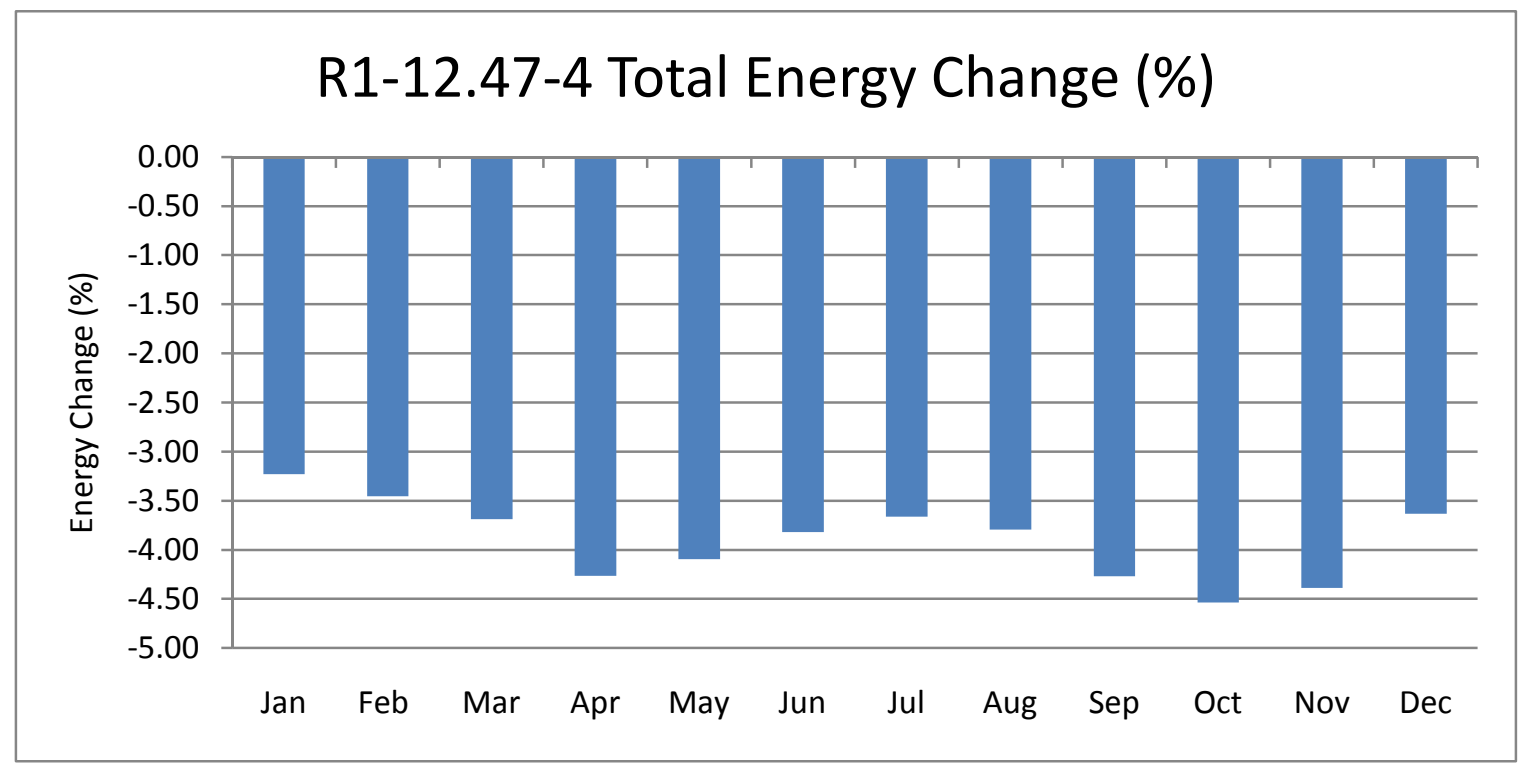

Figure 6.26: R1-12.47-4 Total Energy Change (\%) 


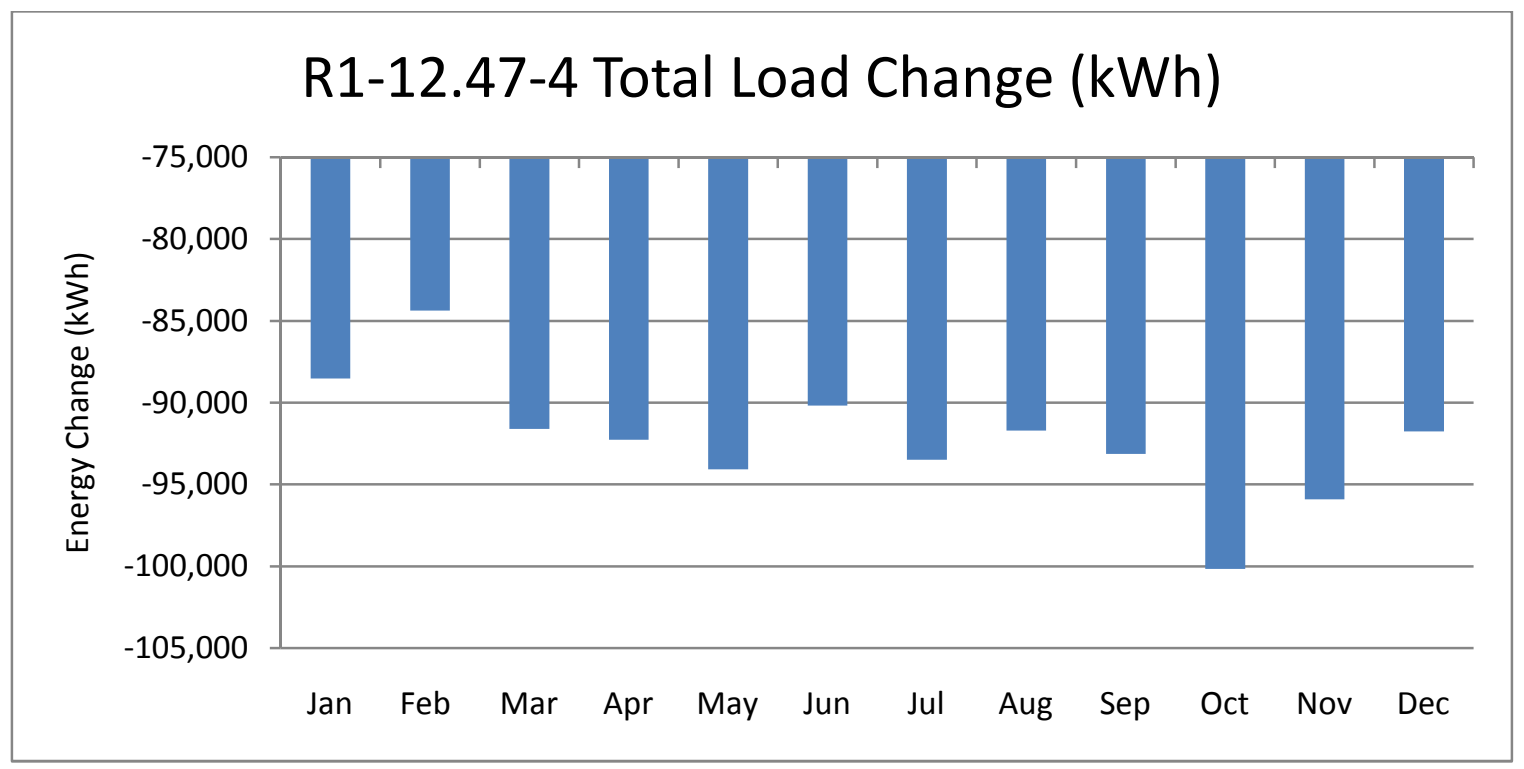

Figure 6.27: R1-12.47-4 Total Load Change (kWh)

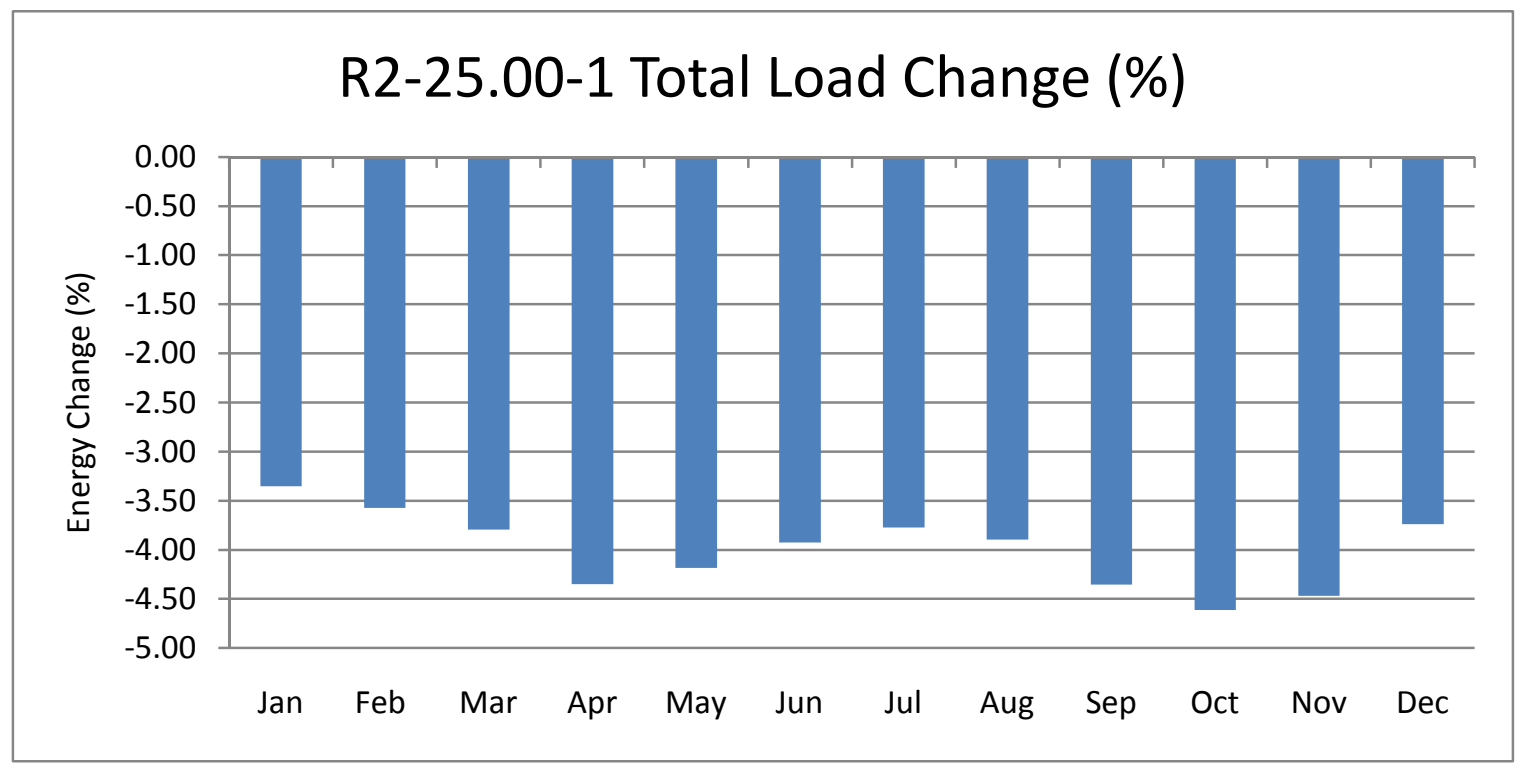

Figure 6.28: R1-12.47-4 Total Load Change (\%) 


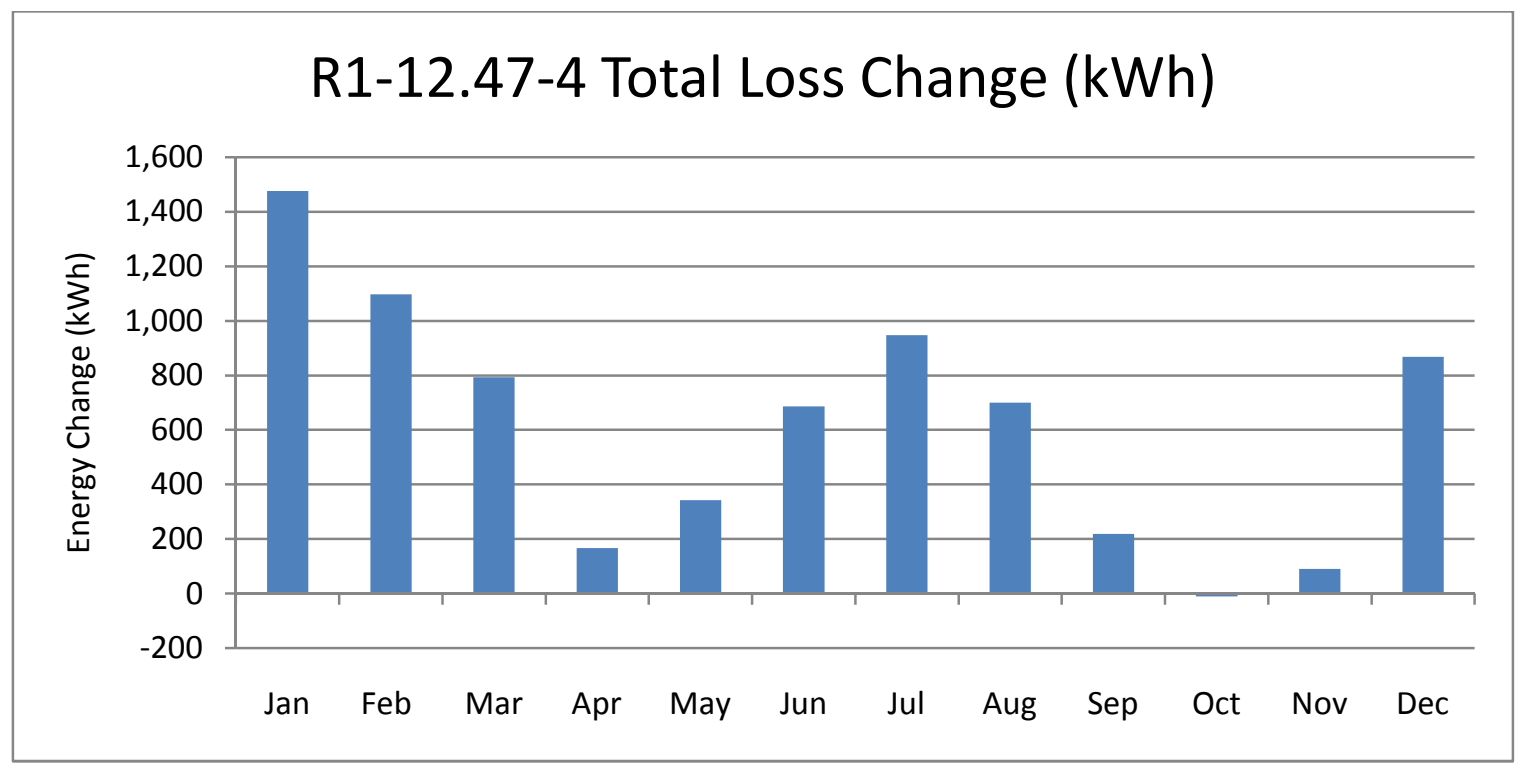

Figure 6.29: R1-12.47-4 Total Loss Change (kWh)

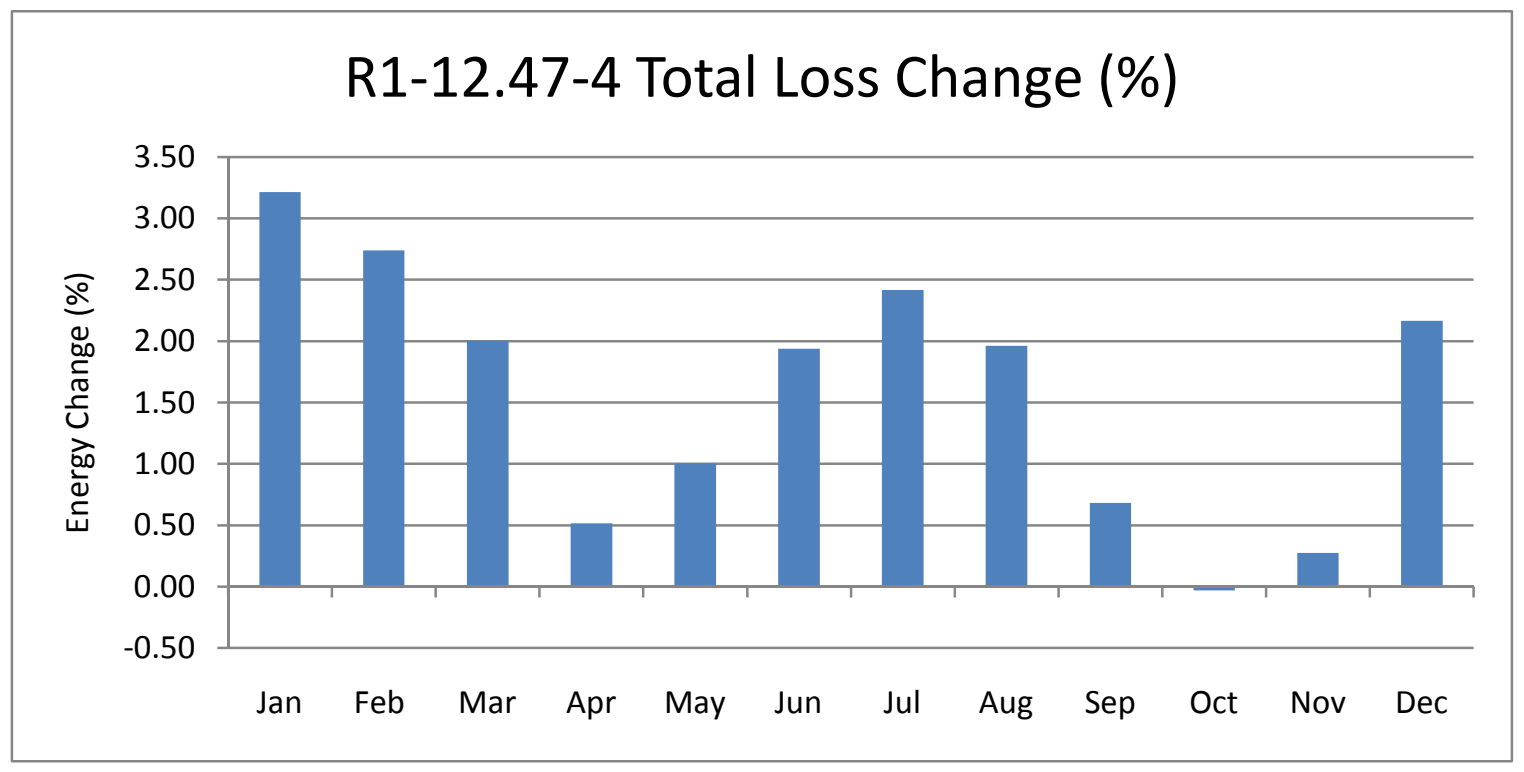

Figure 6.30: R1-12.47-4 Total Loss Change (\%) 


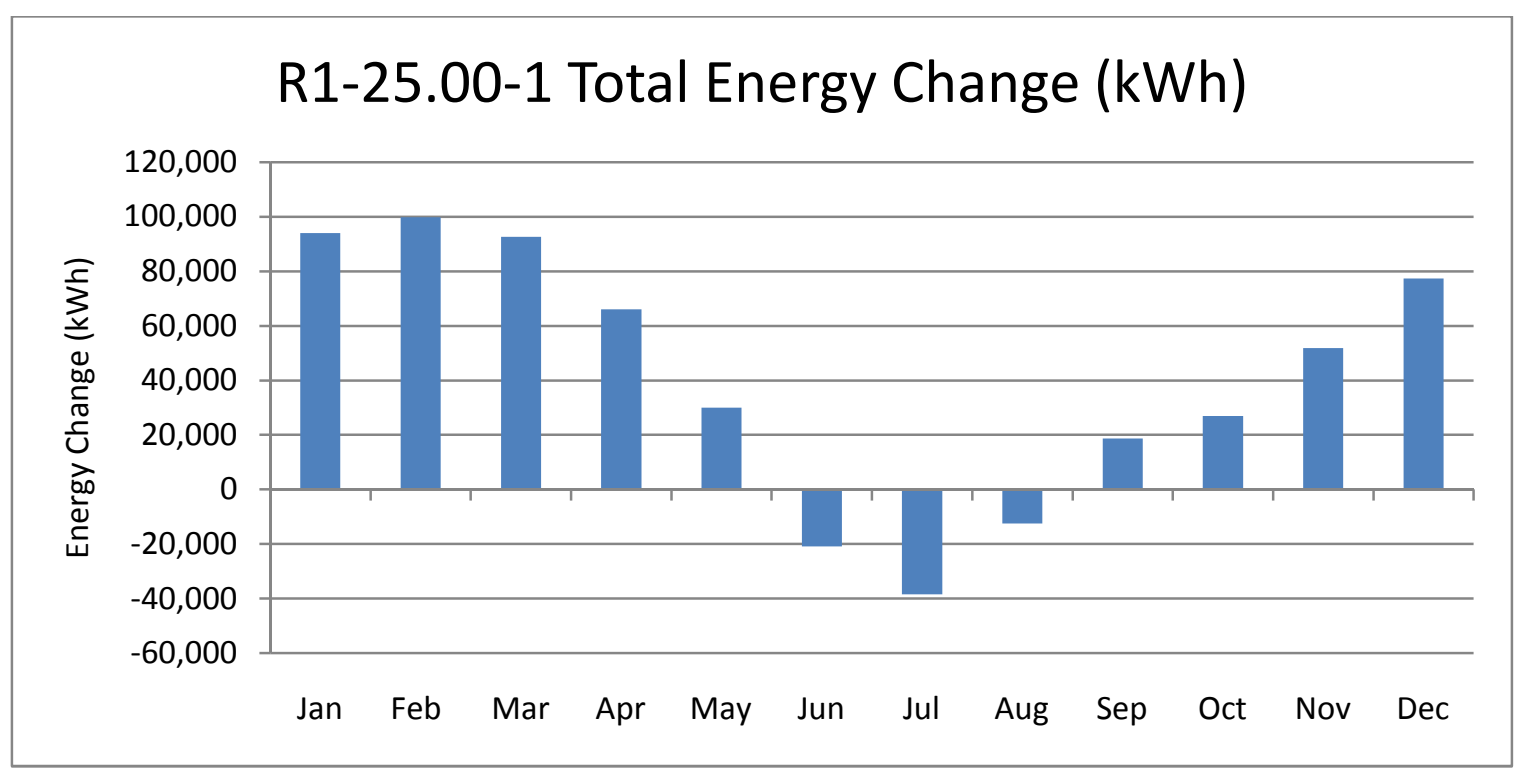

Figure 6.31: R1-25.00-1 Total Energy Change (kWh)

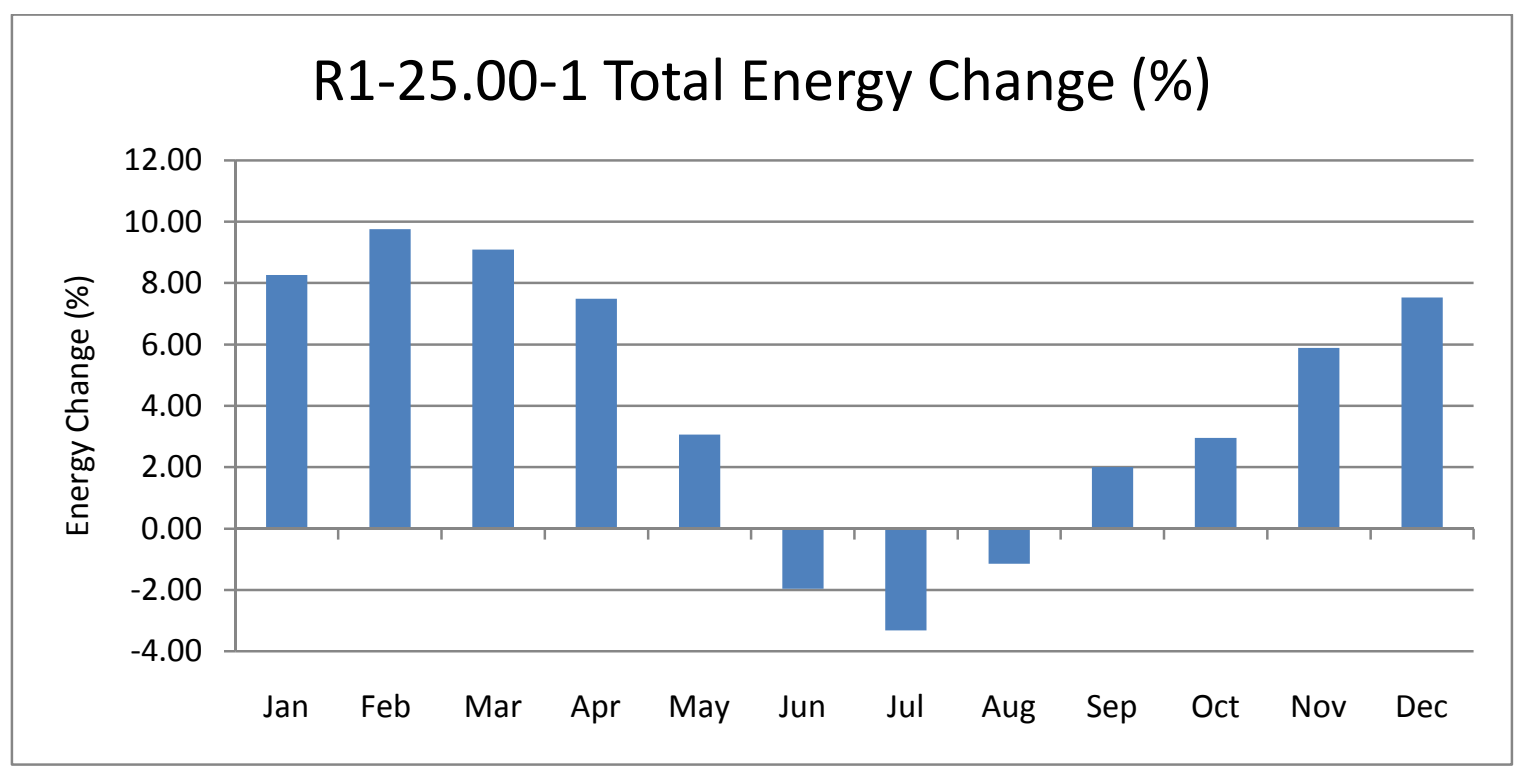

Figure 6.32: R1-25.00-1 Total Energy Change (\%) 


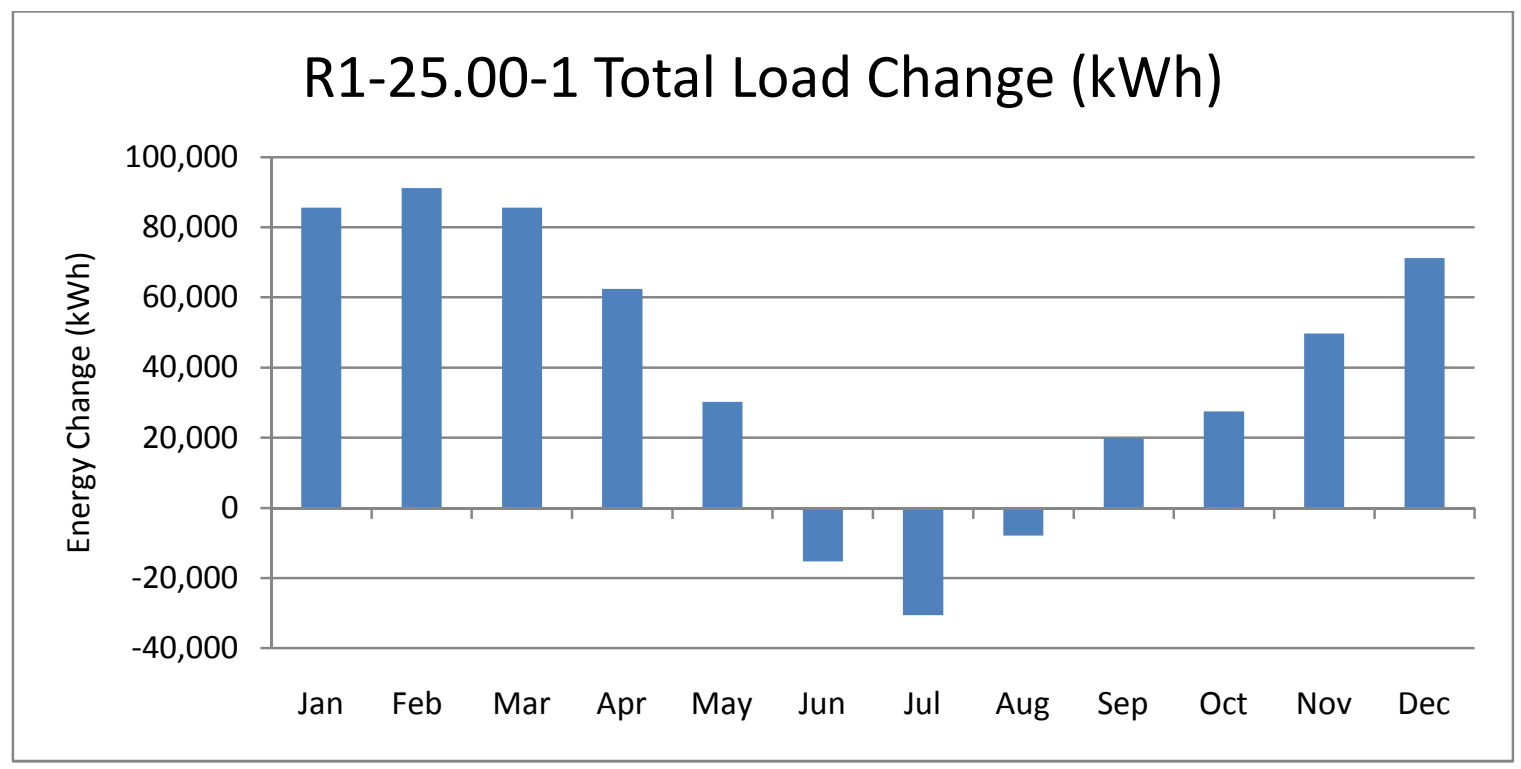

Figure 6.33: R1-25.00-1 Total Load Change (kWh)

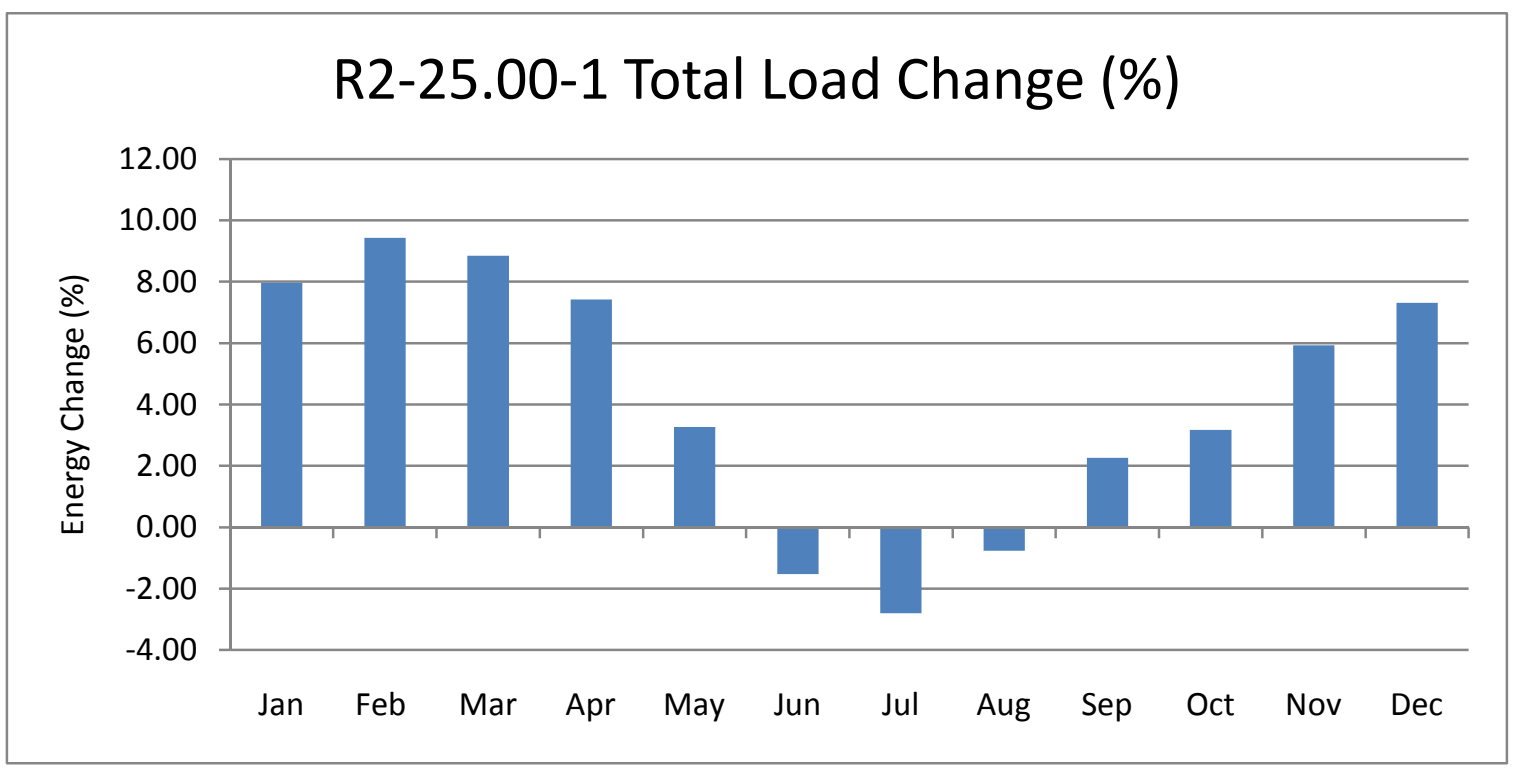

Figure 6.34: R1-25.00-1 Total Load Change (\%) 


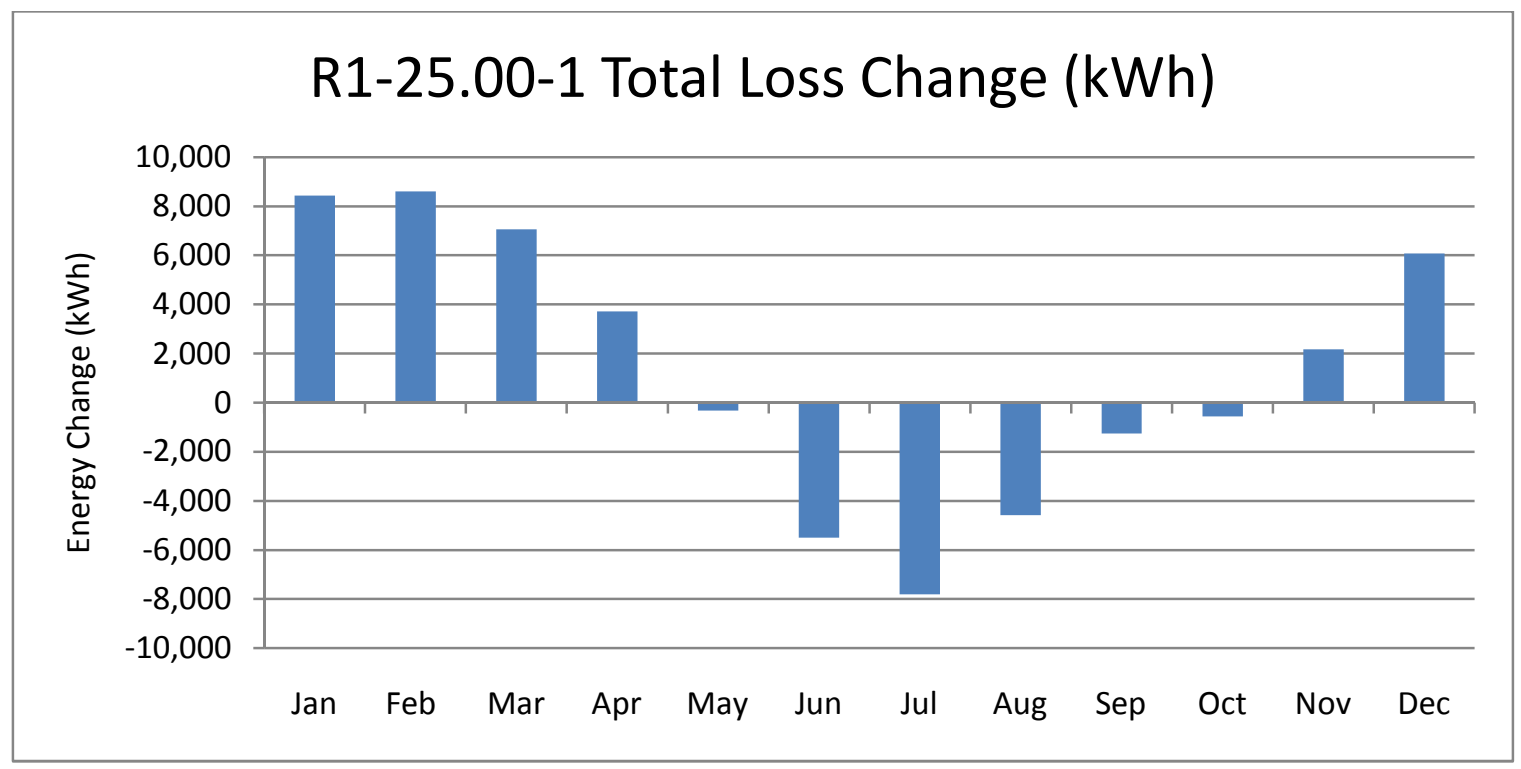

Figure 6.35: R1-25.00-1 Total Loss Change (kWh)

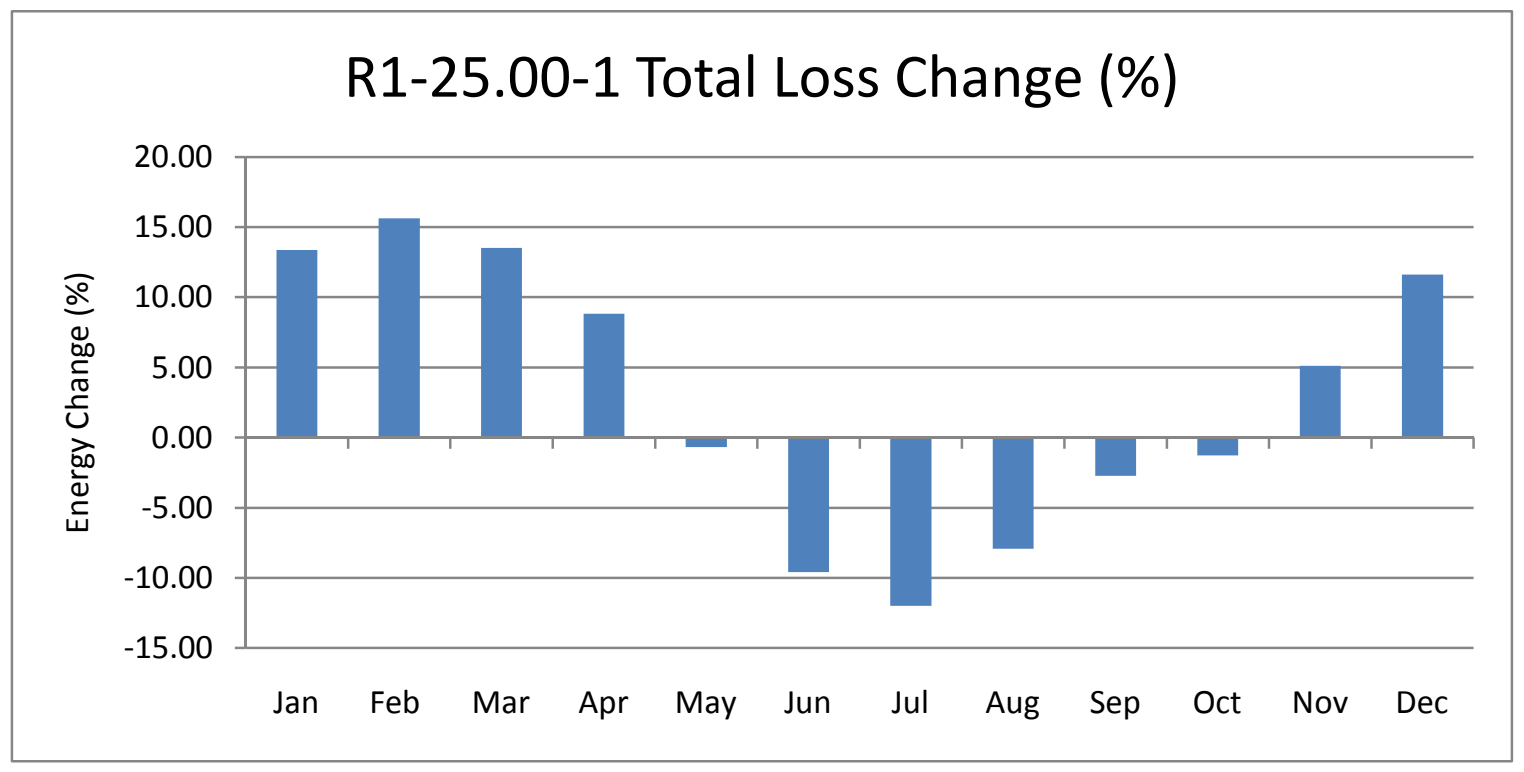

Figure 6.36: R1-25.00-1 Total Loss Change (\%)

\subsection{Region 2: CVR Plots}




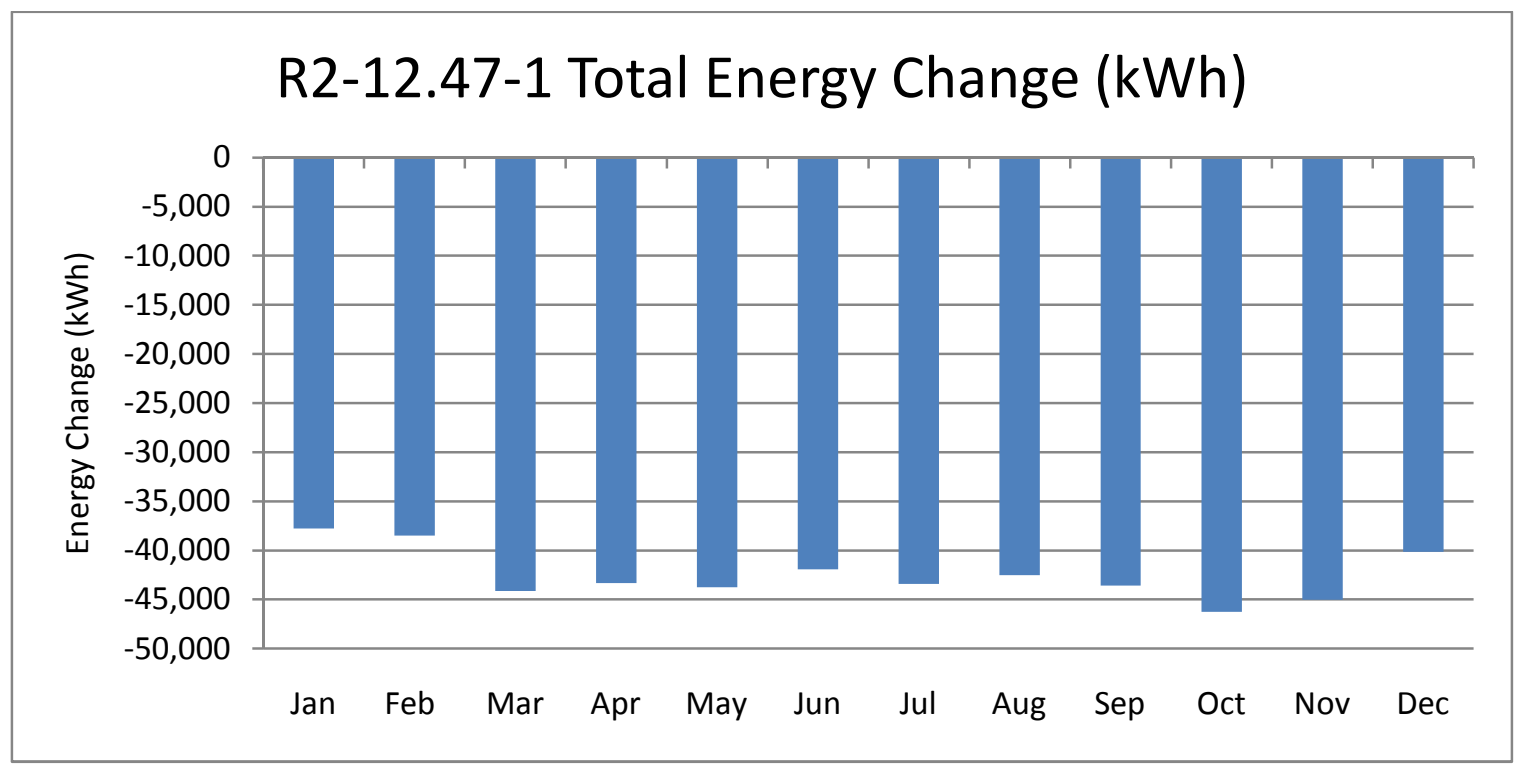

Figure 6.37: R2-12.47-1 Total Energy Change (kWh)

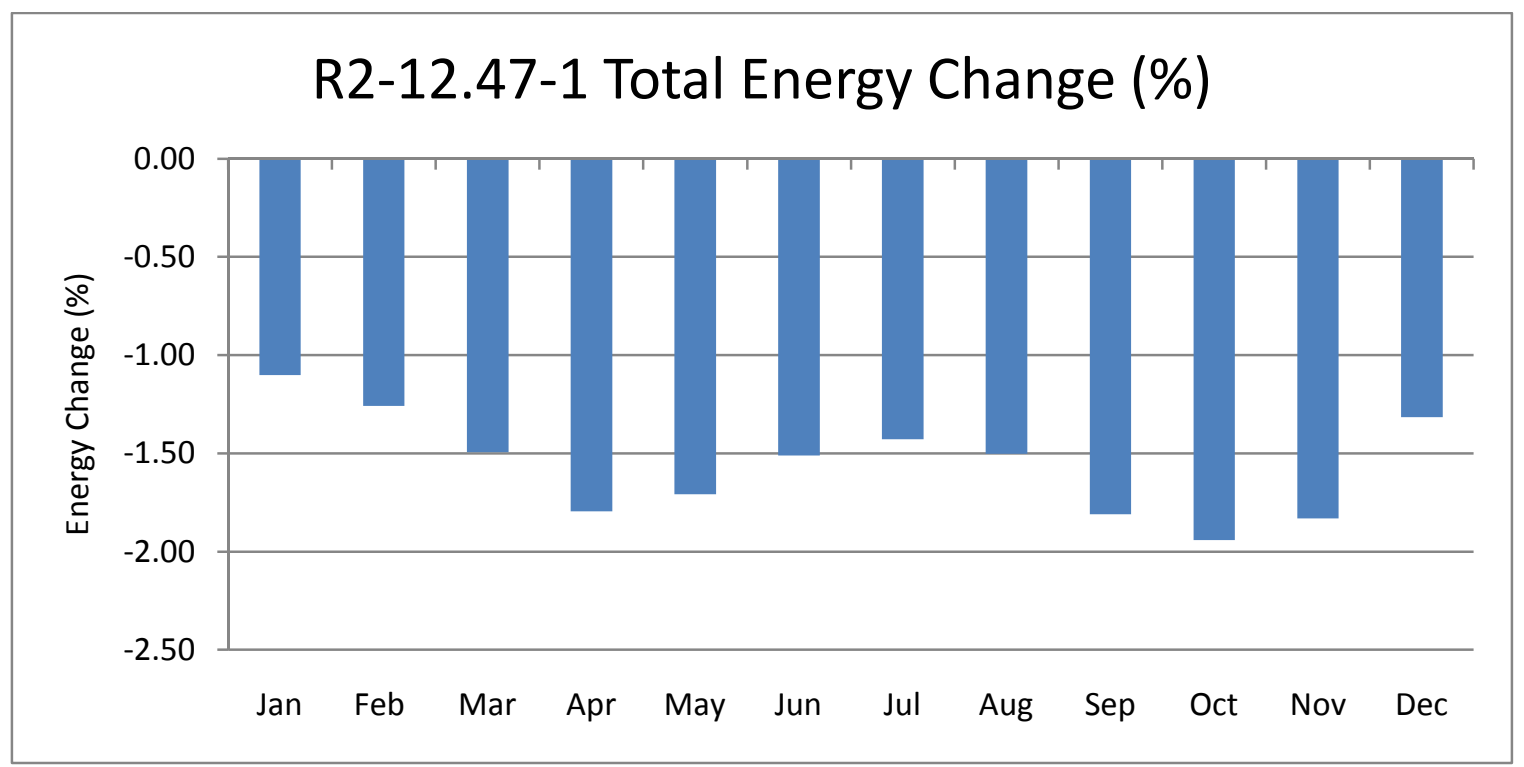

Figure 6.38: R2-12.47-1 Total Energy Change kWh 


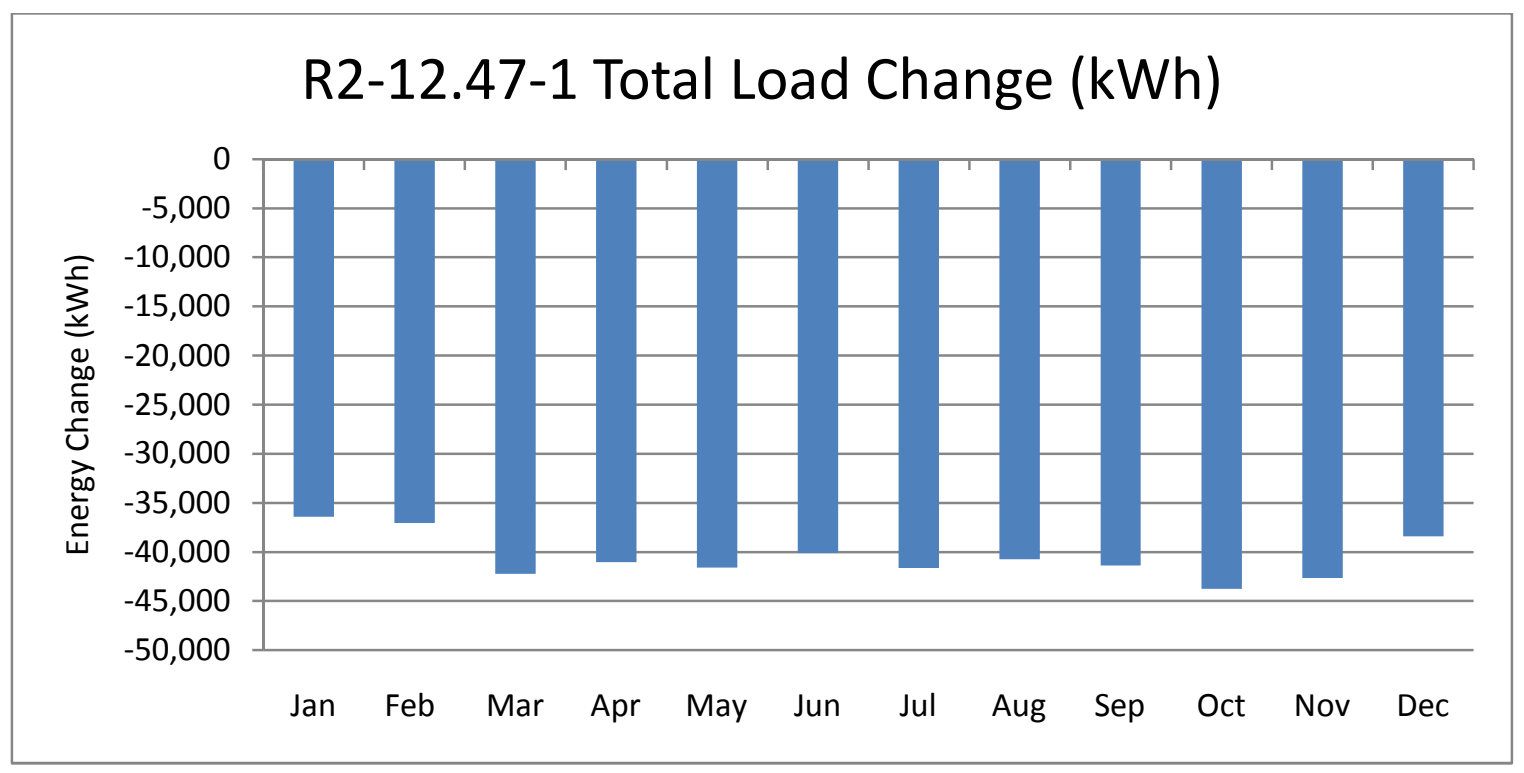

Figure 6.39: R2-12.47-1 Total Load Change (kWh)

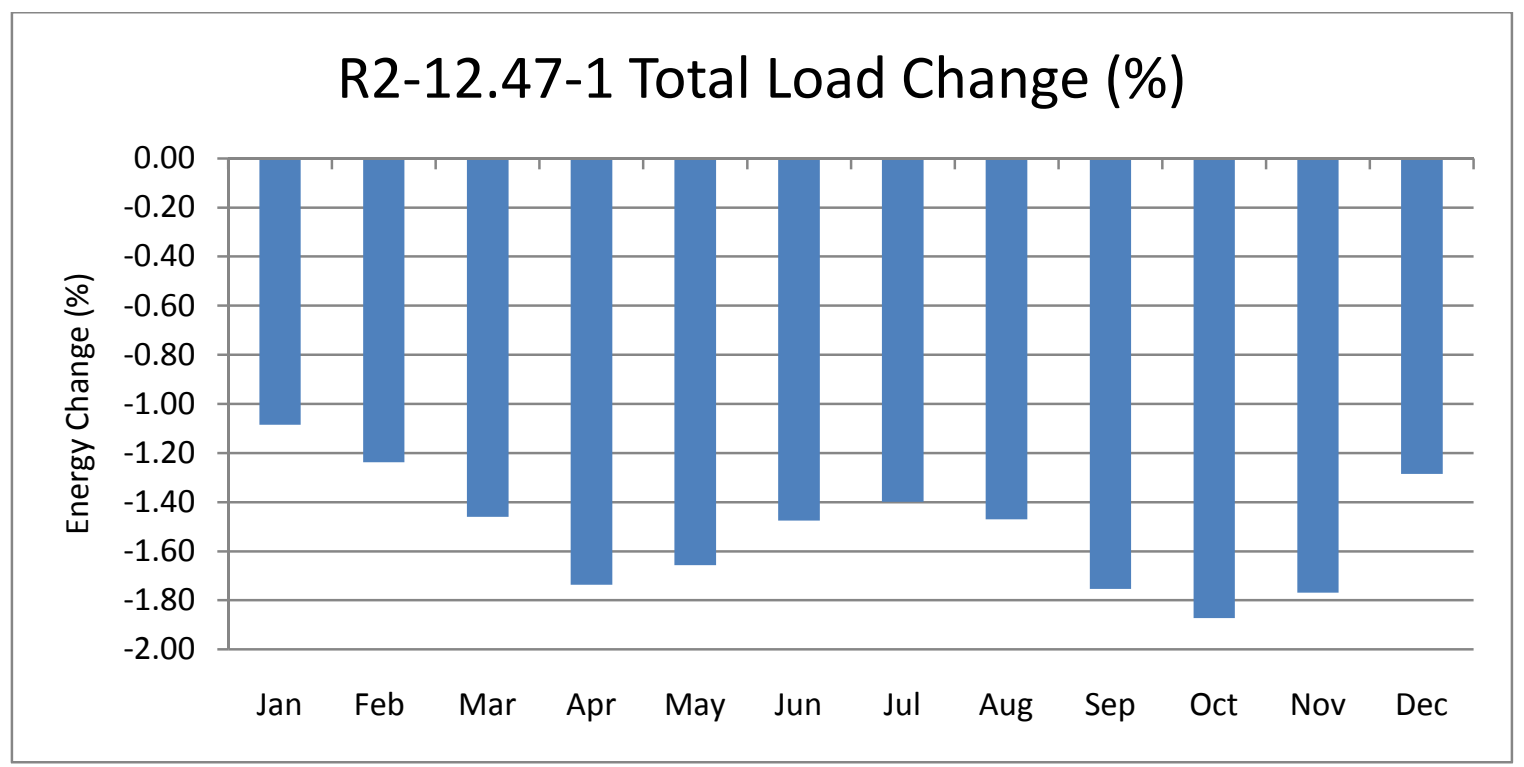

Figure 6.40: R2-12.47-1 Total Load Change (\%) 


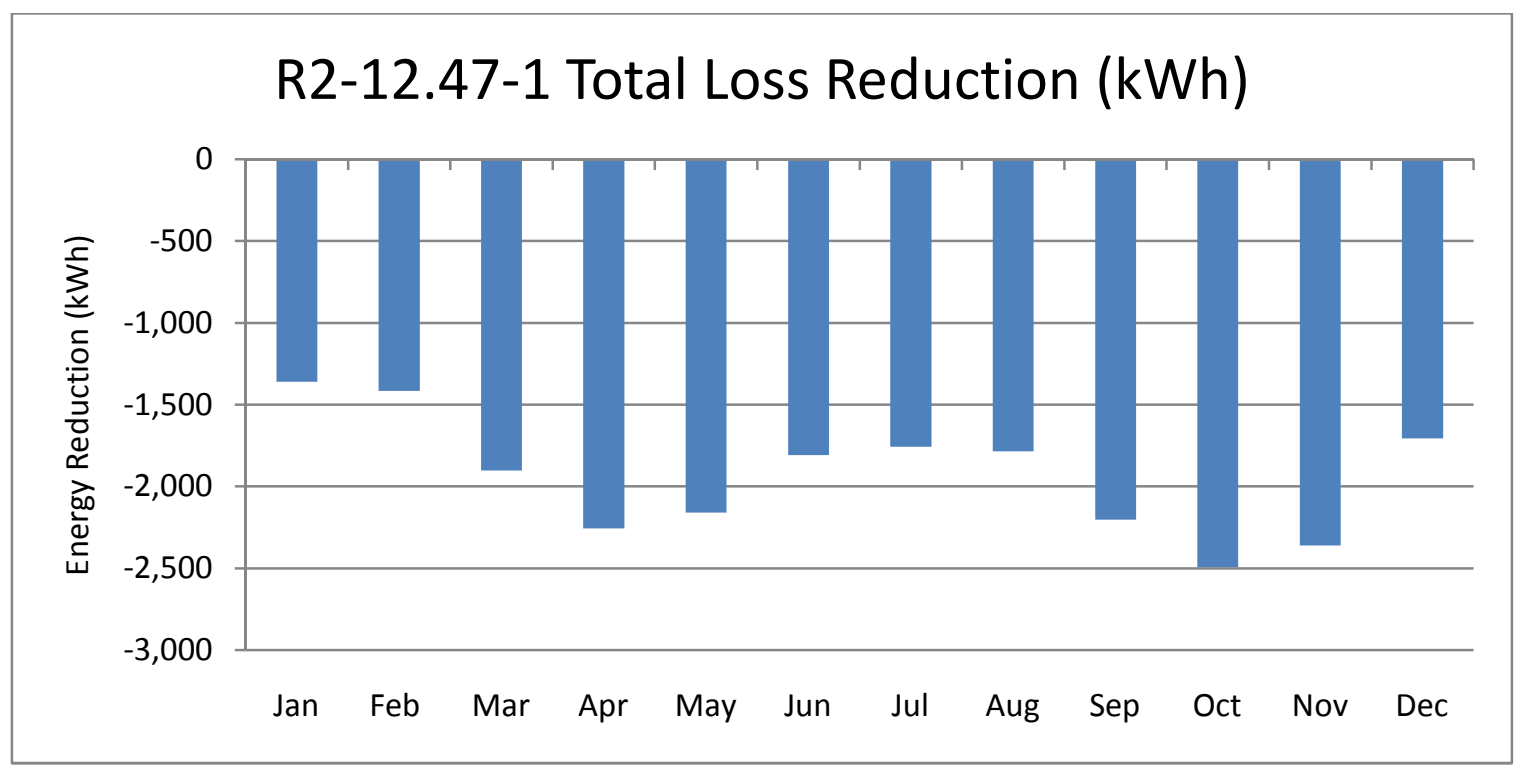

Figure 6.41: R2-12.47-1 Total Loss Change (kWh)

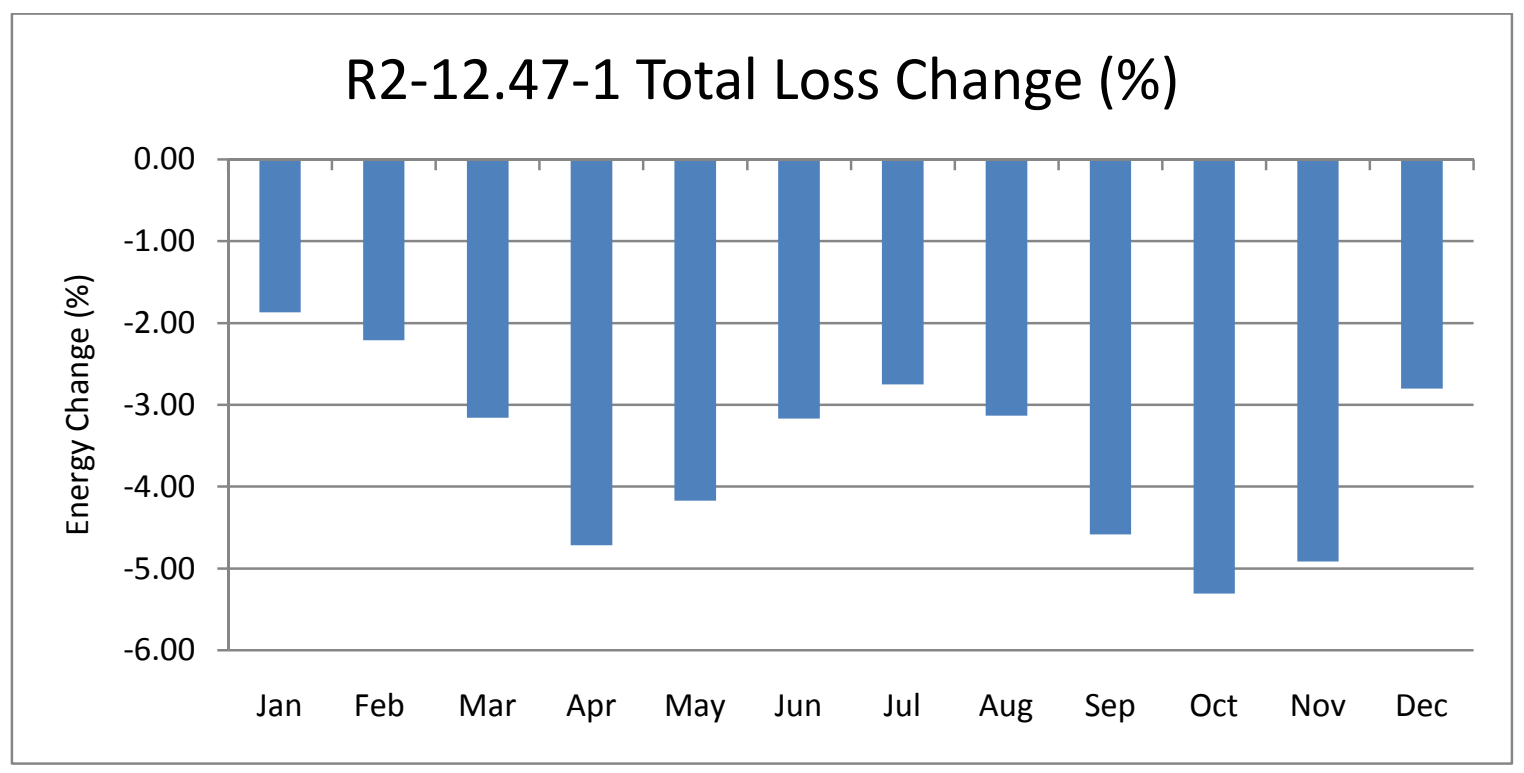

Figure 6.42: R2-12.47-1 Total Loss Change (\%) 


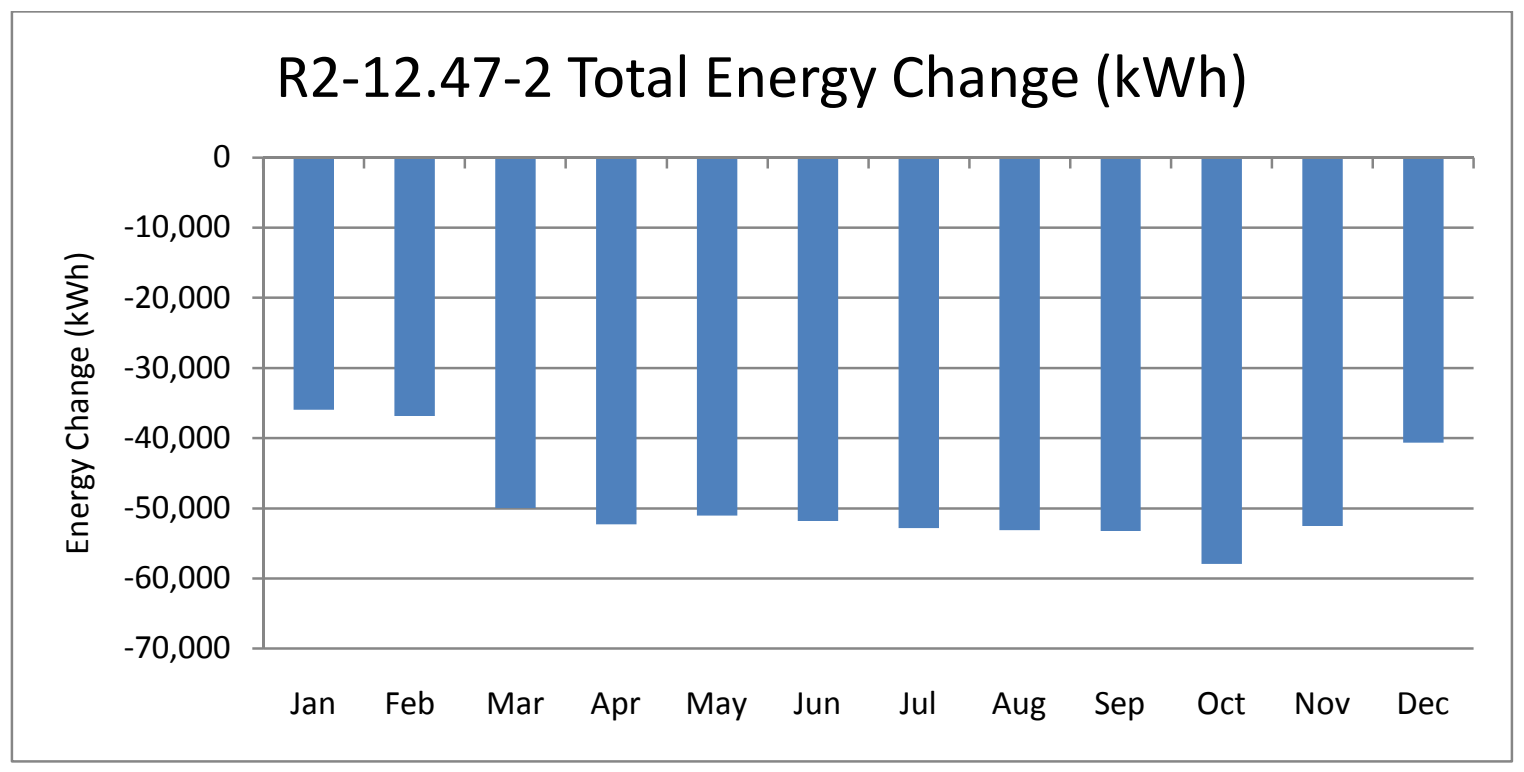

Figure 6.43: R2-12.47-2 Total Energy Change (kWh)

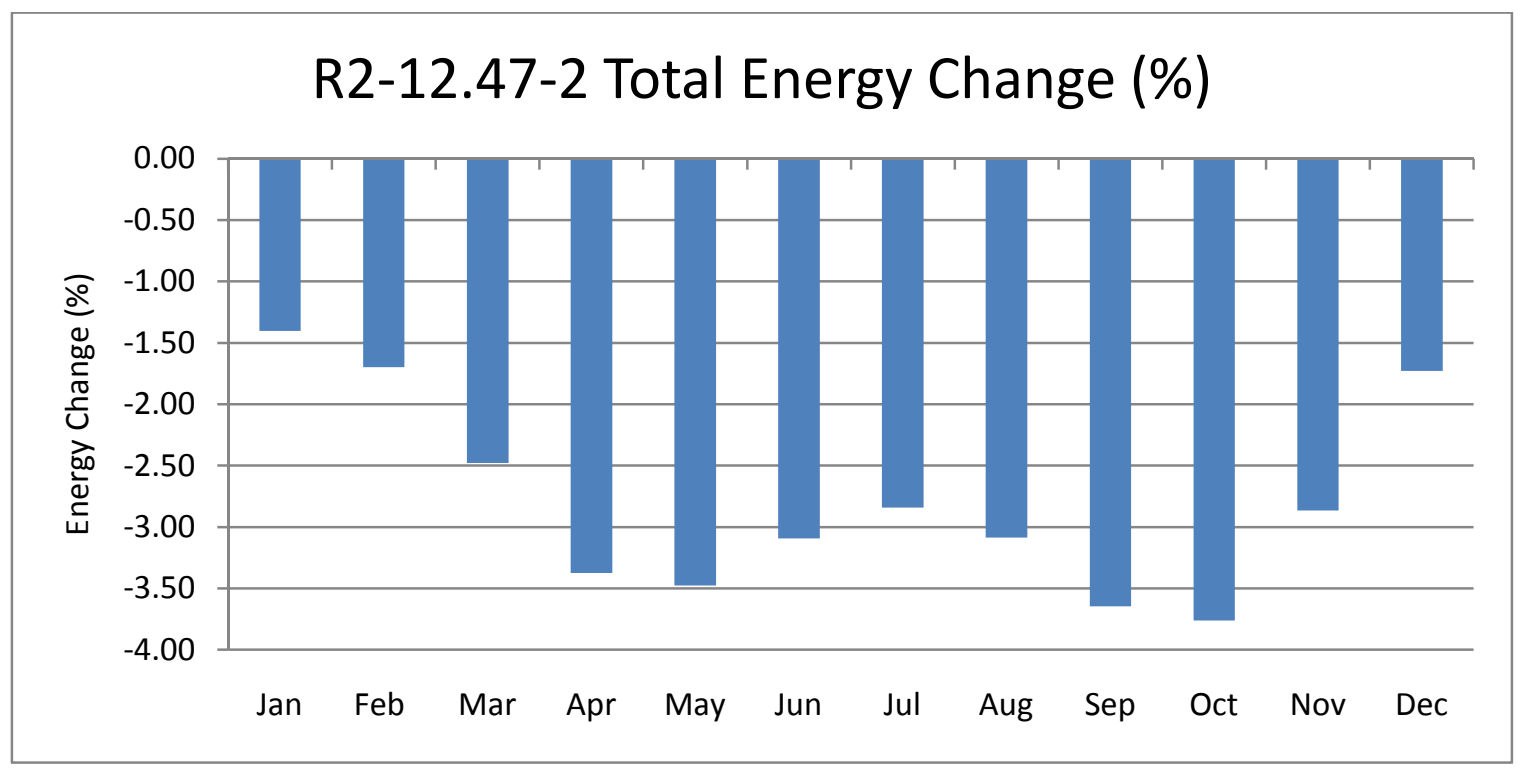

Figure 6.44: R2-12.47-2 Total Energy Change (\%) 


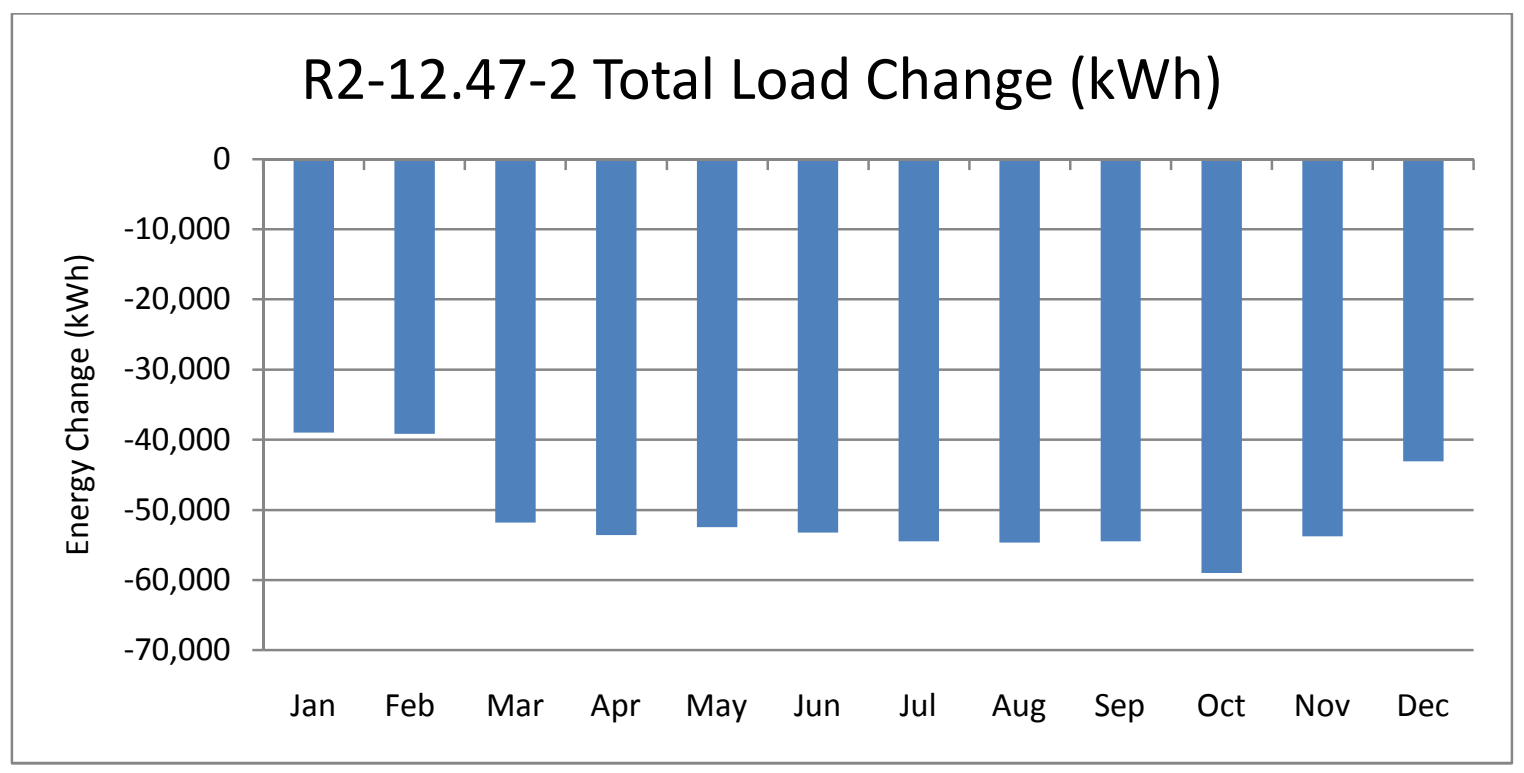

Figure 6.45: R2-12.47-2 Total Load Change (kWh)

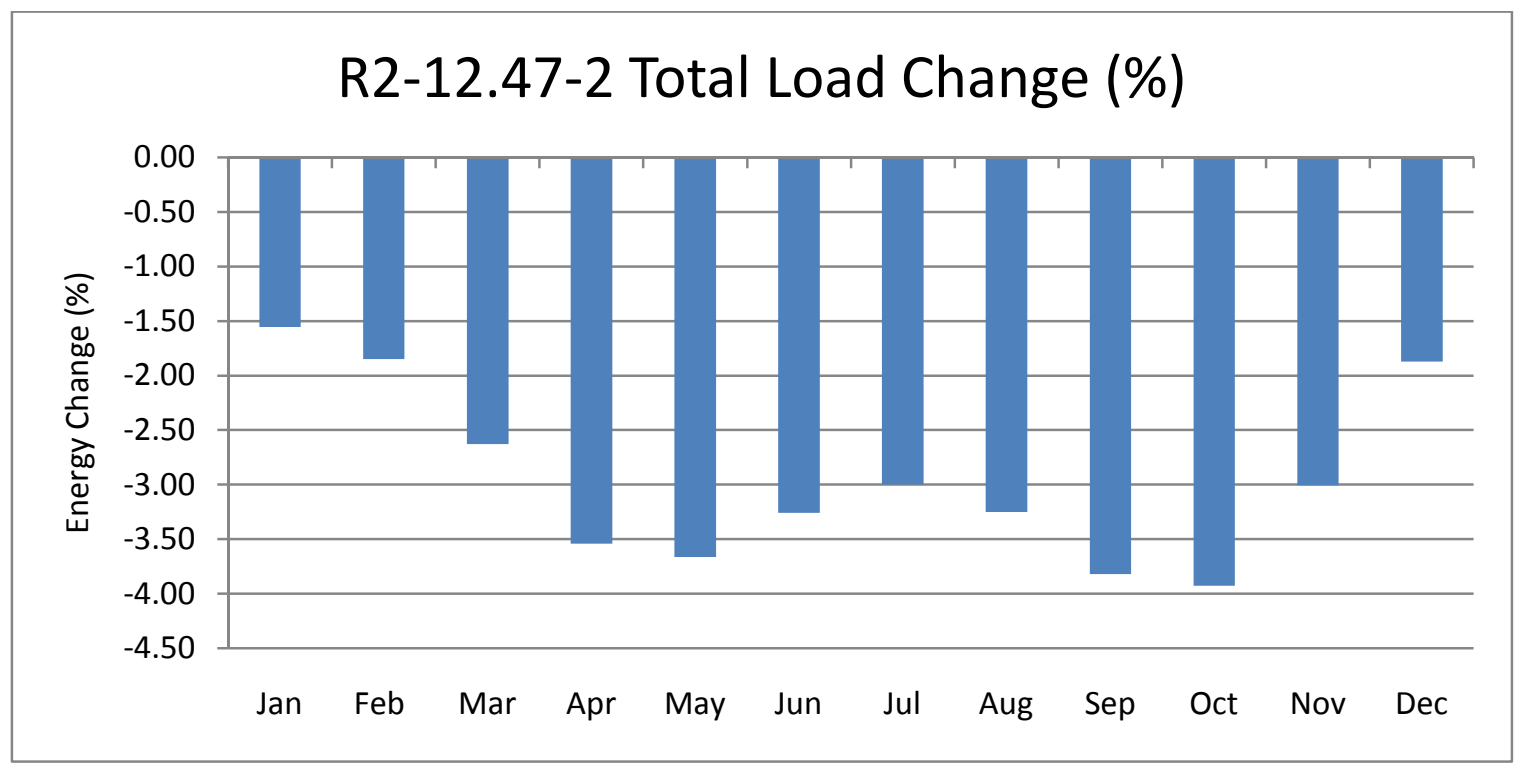

Figure 6.46: R2-12.47-2 Total Load Change (\%) 


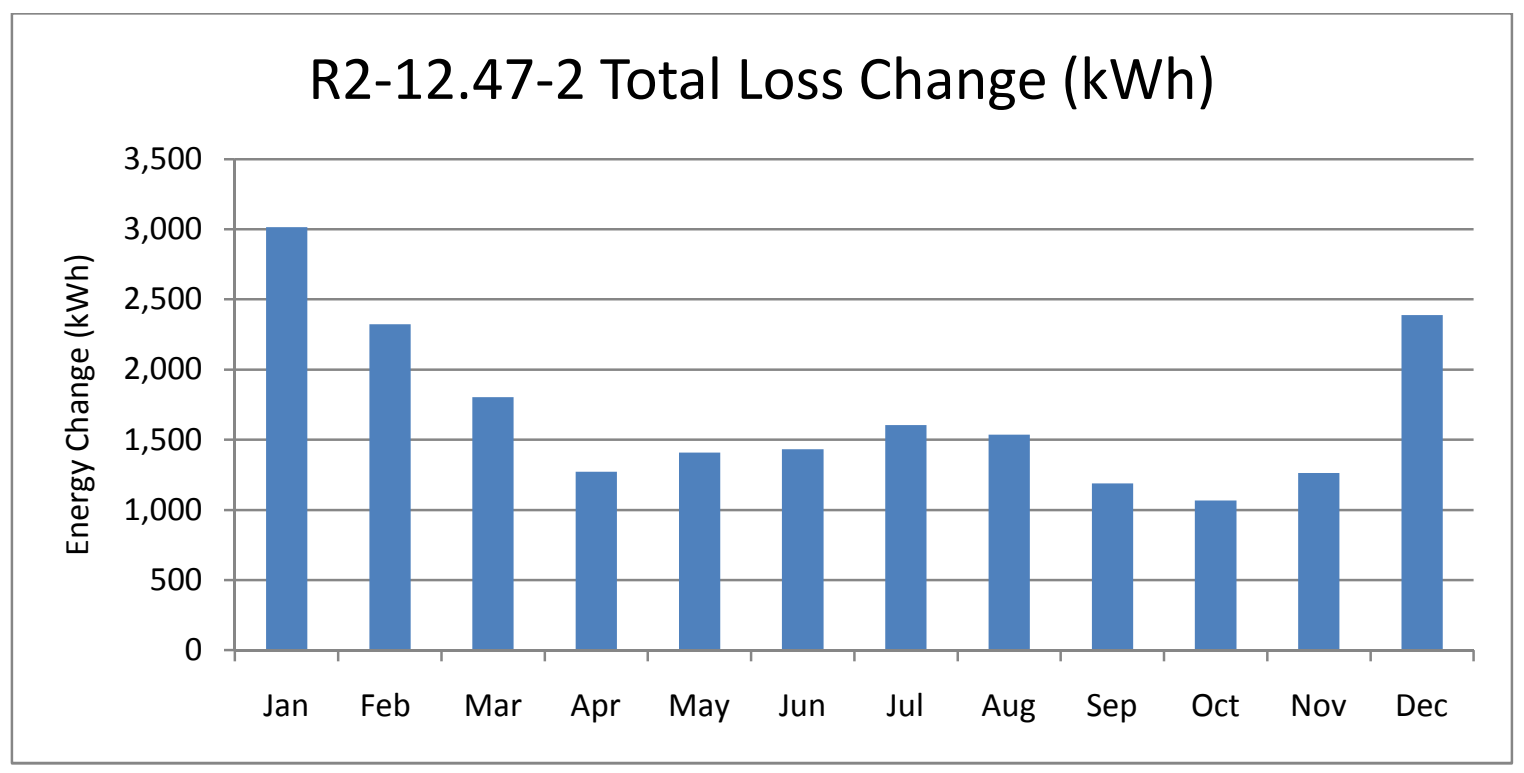

Figure 6.47: R2-12.47-2 Total Loss Change (kWh)

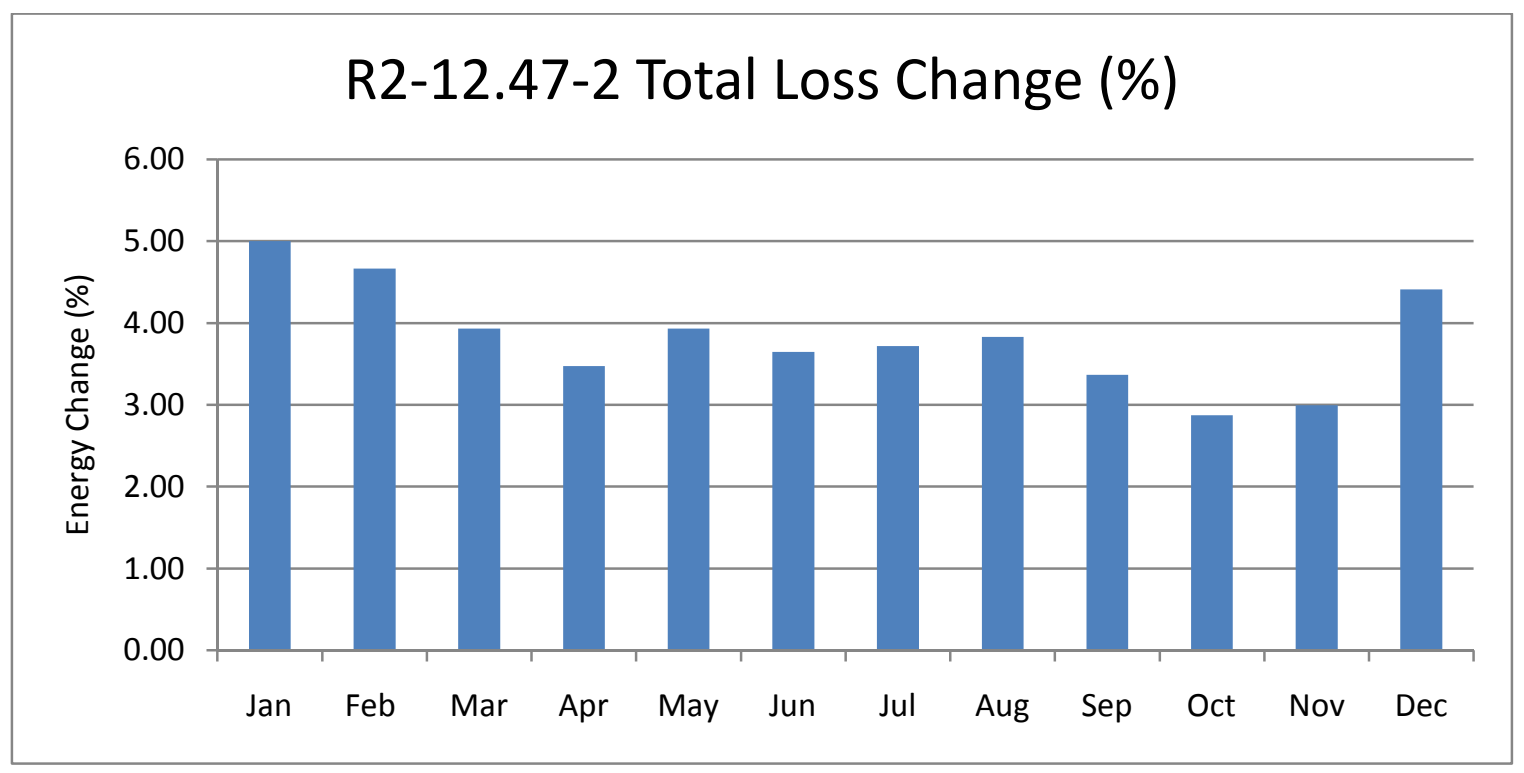

Figure 6.48: R2-12.47-2 Total Loss Change (\%) 


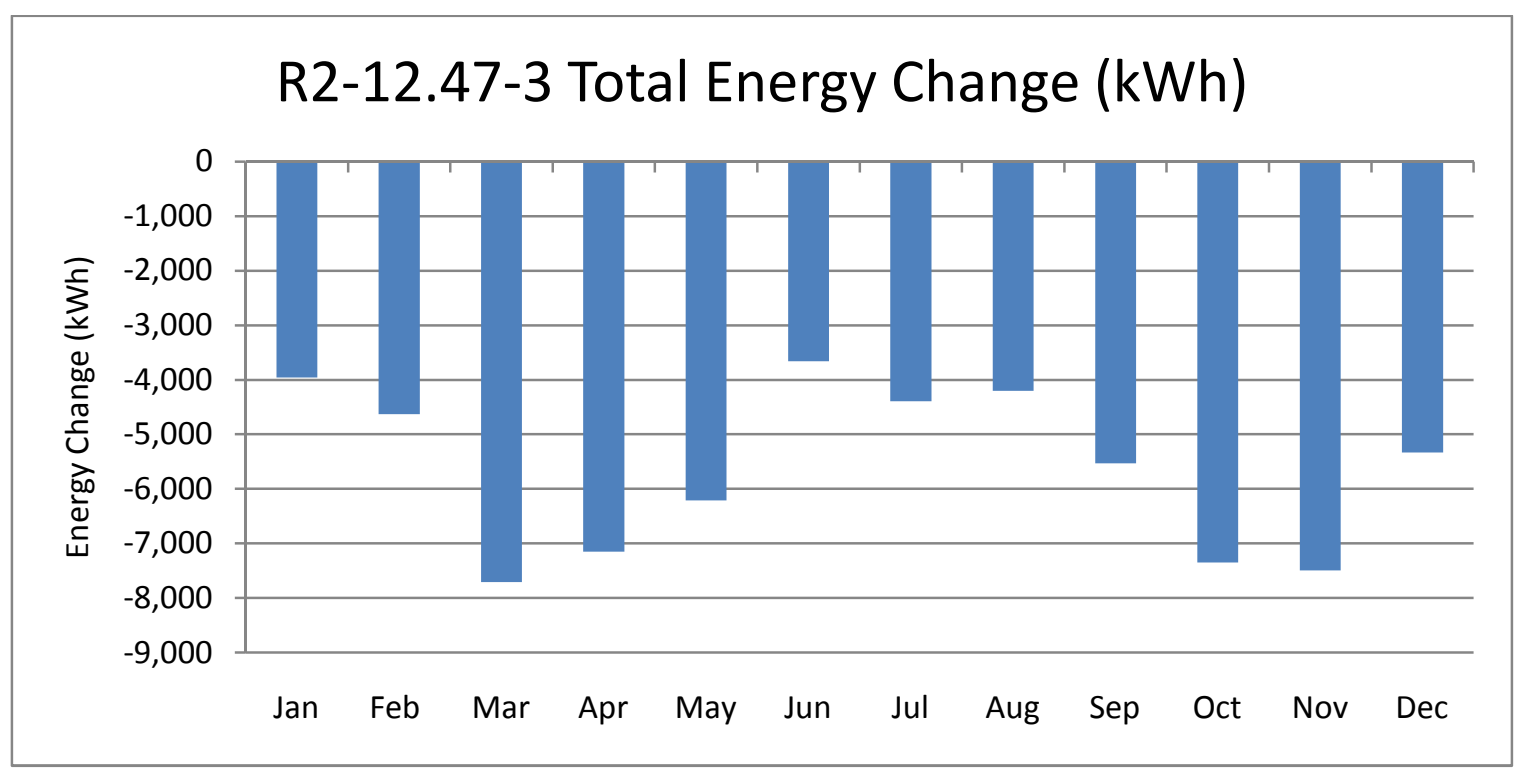

Figure 6.49: R2-12.47-3 Total Energy Change (kWh)

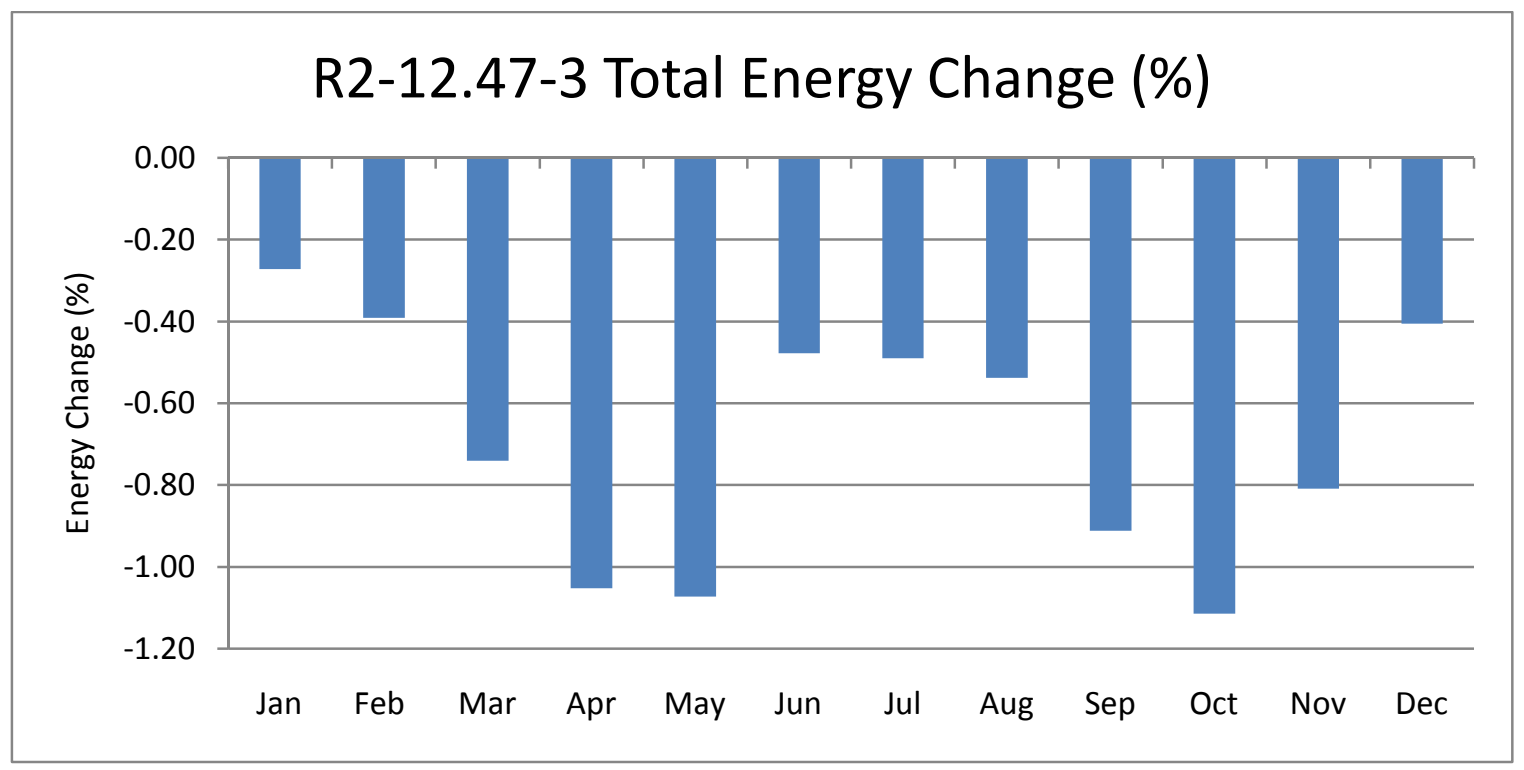

Figure 6.50: R2-12.47-3 Total Energy Change (\%) 


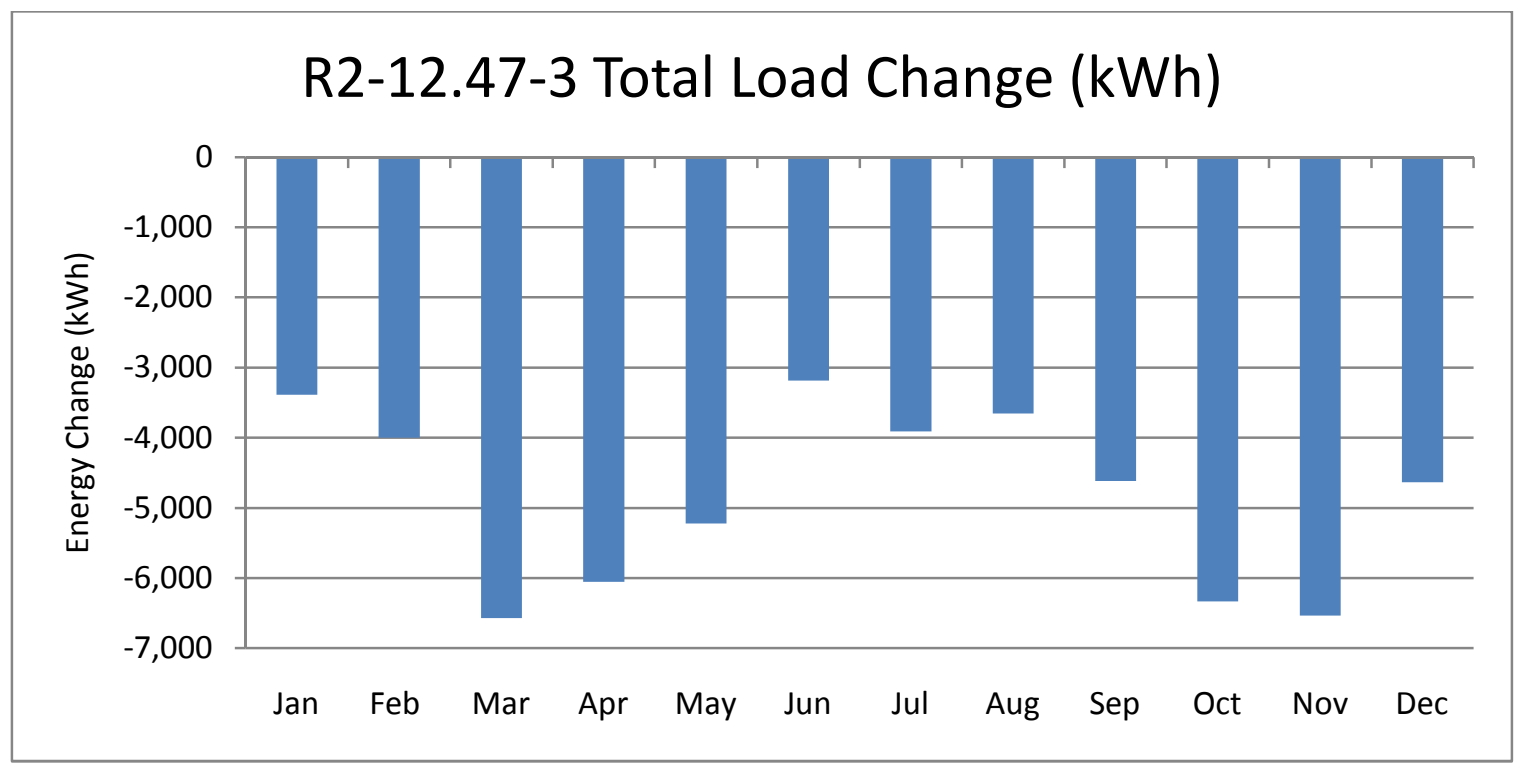

Figure 6.51: R2-12.47-3 Total Load Change (kWh)

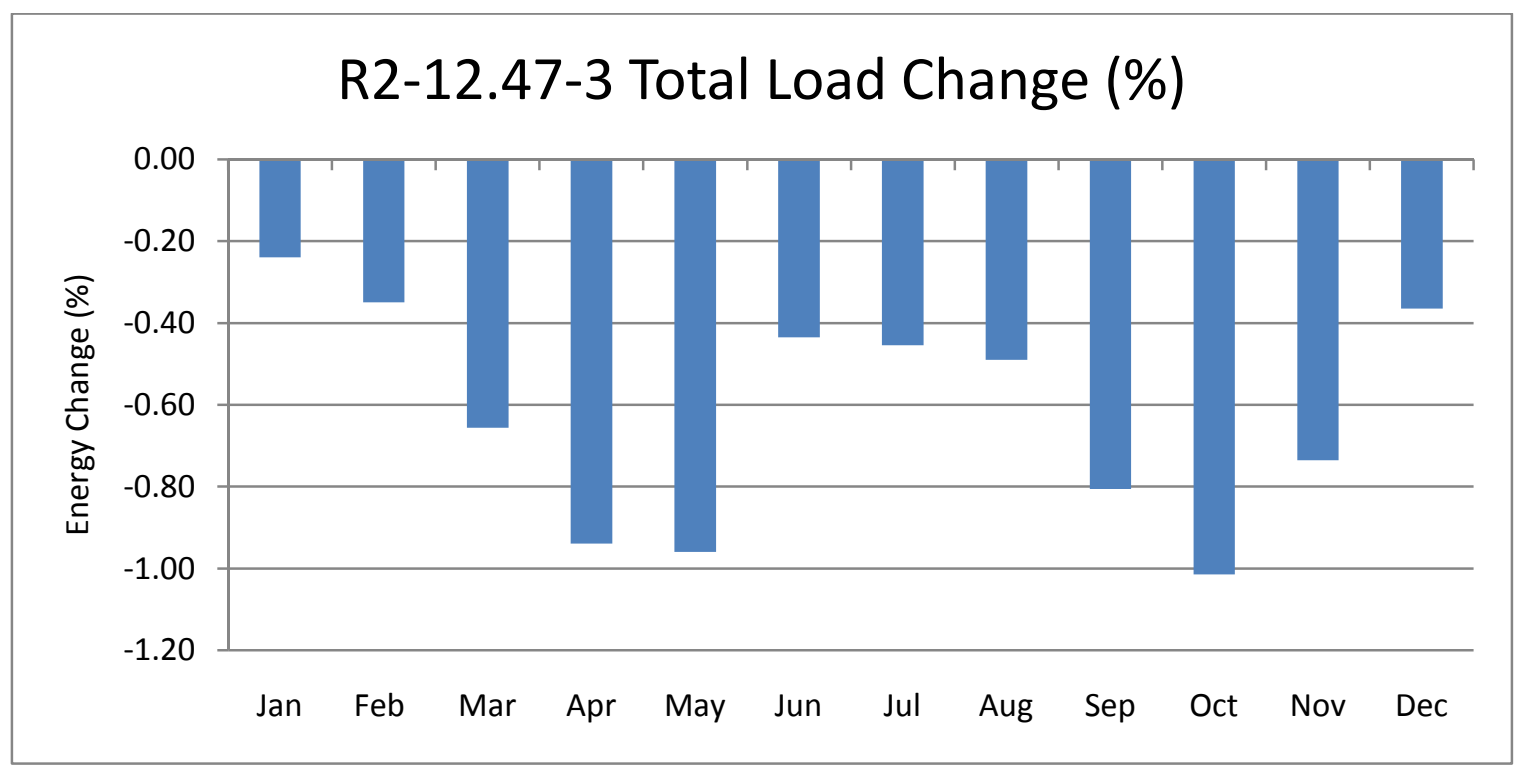

Figure 6.52: R2-12.47-3 Total Load Change (\%) 


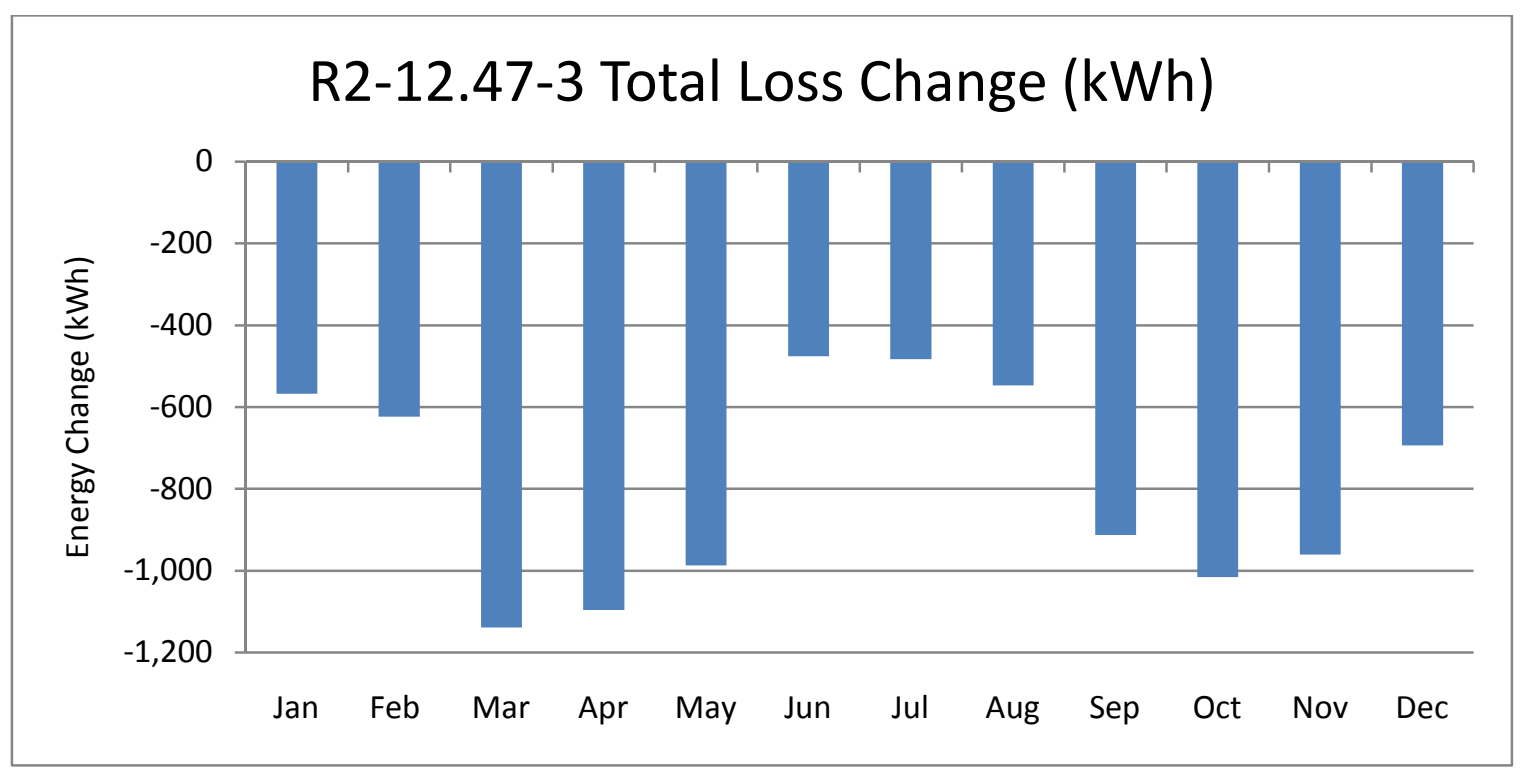

Figure 6.53: R2-12.47-3 Total Loss Change (kWh)

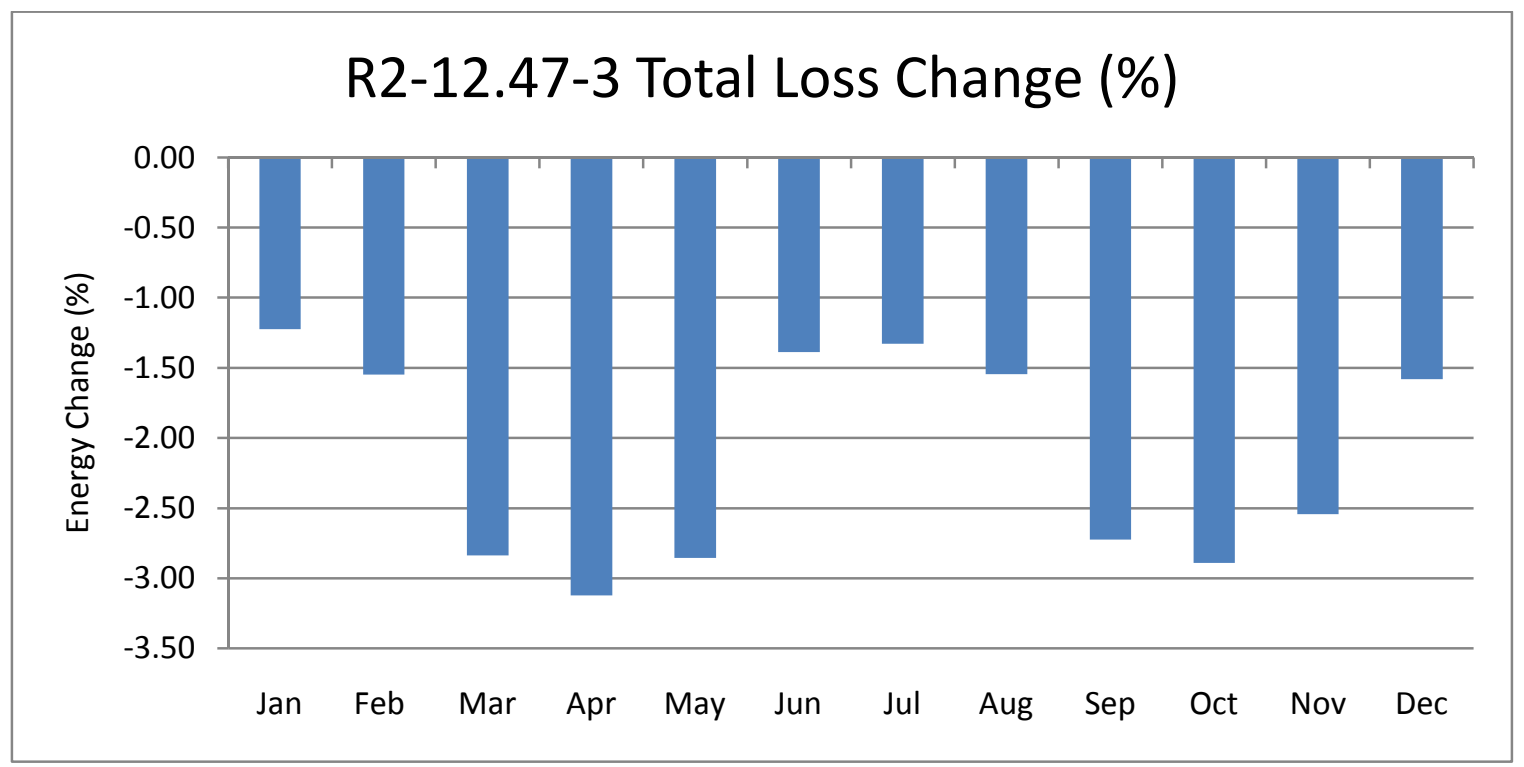

Figure 6.54: R2-12.47-3 Total Loss Change (\%) 


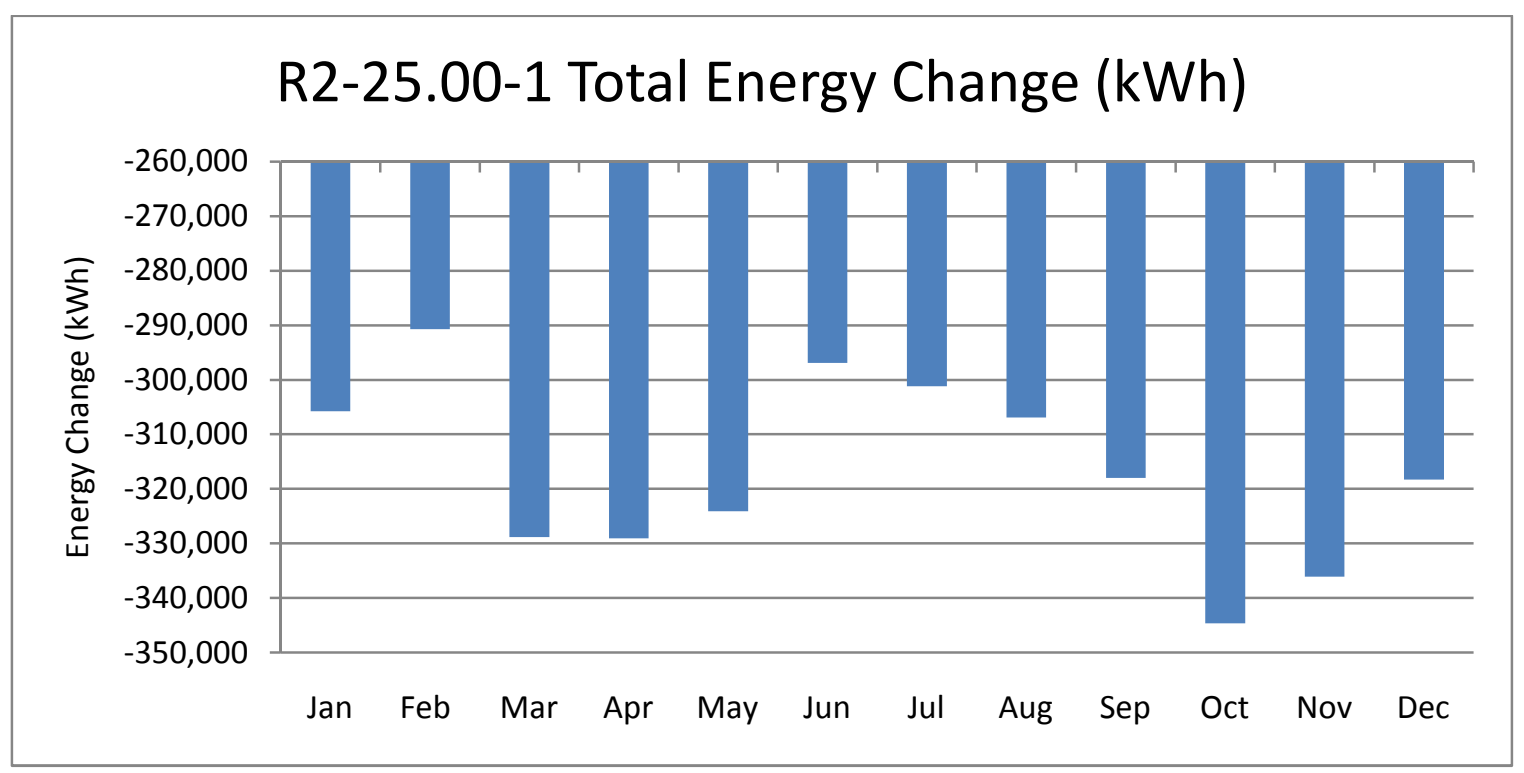

Figure 6.55: R2-25.00-1 Total Energy Change (kWh)

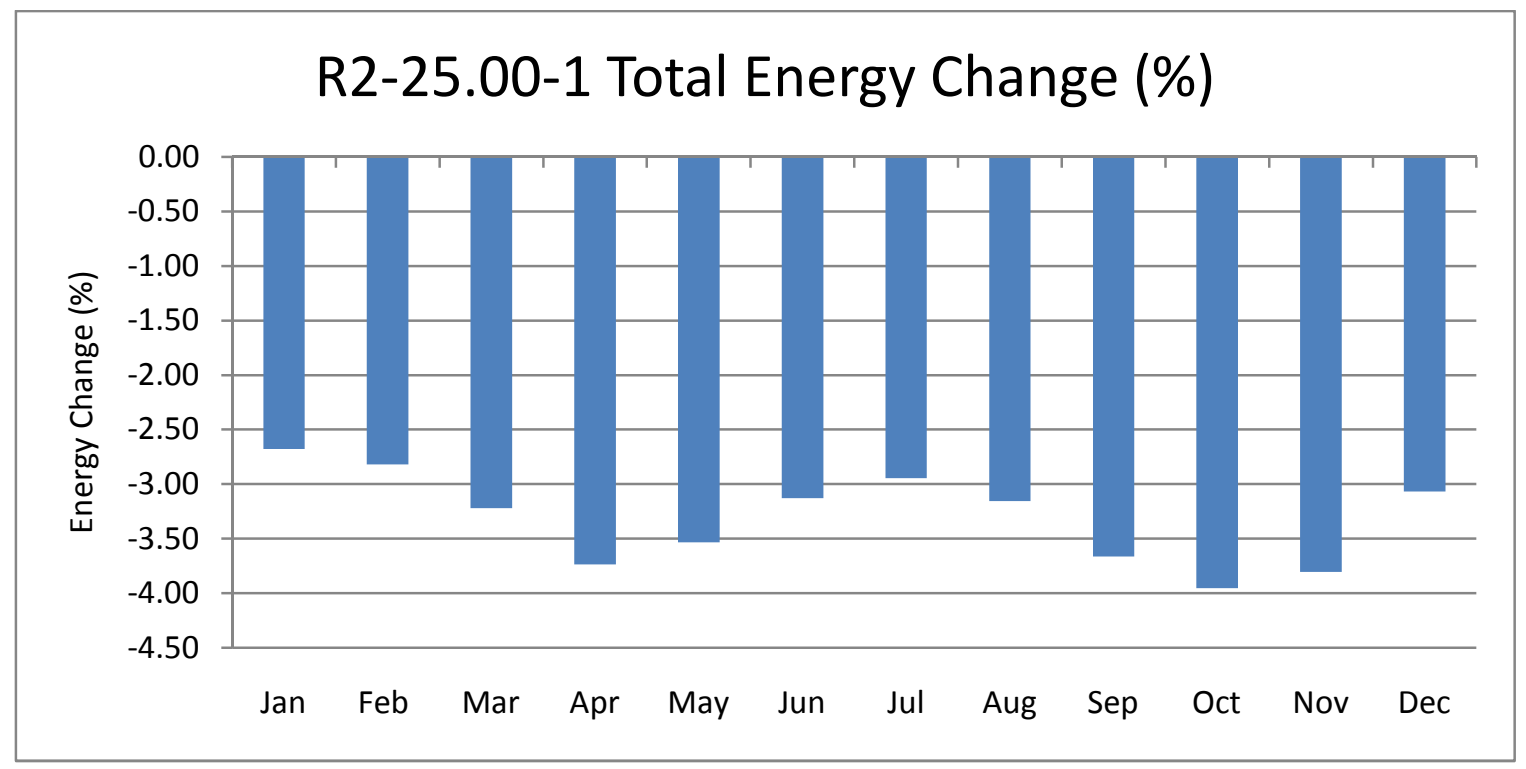

Figure 6.56: R2-25.00-1 Total Energy Change (\%) 


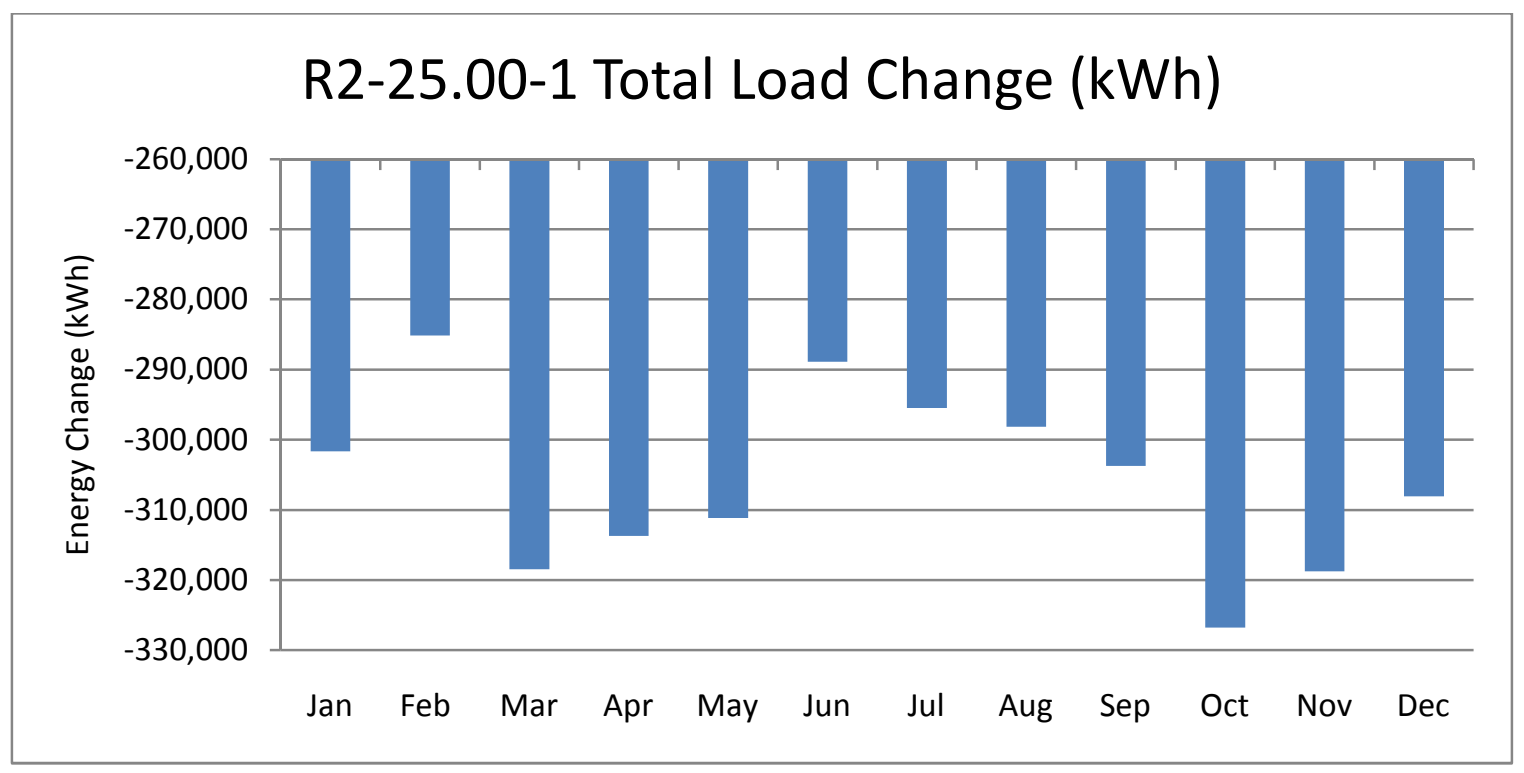

Figure 6.57: R2-25.00-1 Total Load Change (kWh)

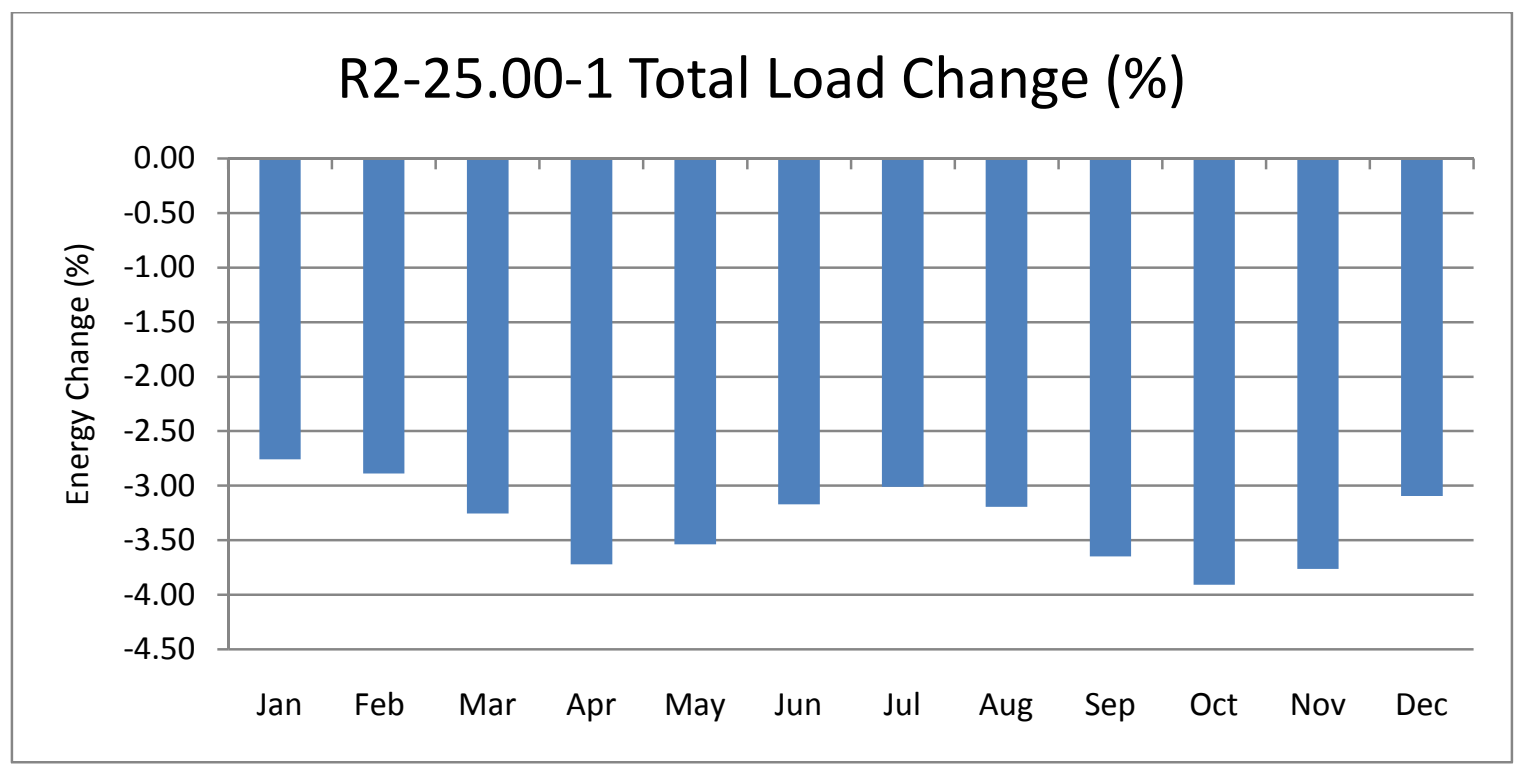

Figure 6.58: R2-25.00-1 Total Load Change (\%) 


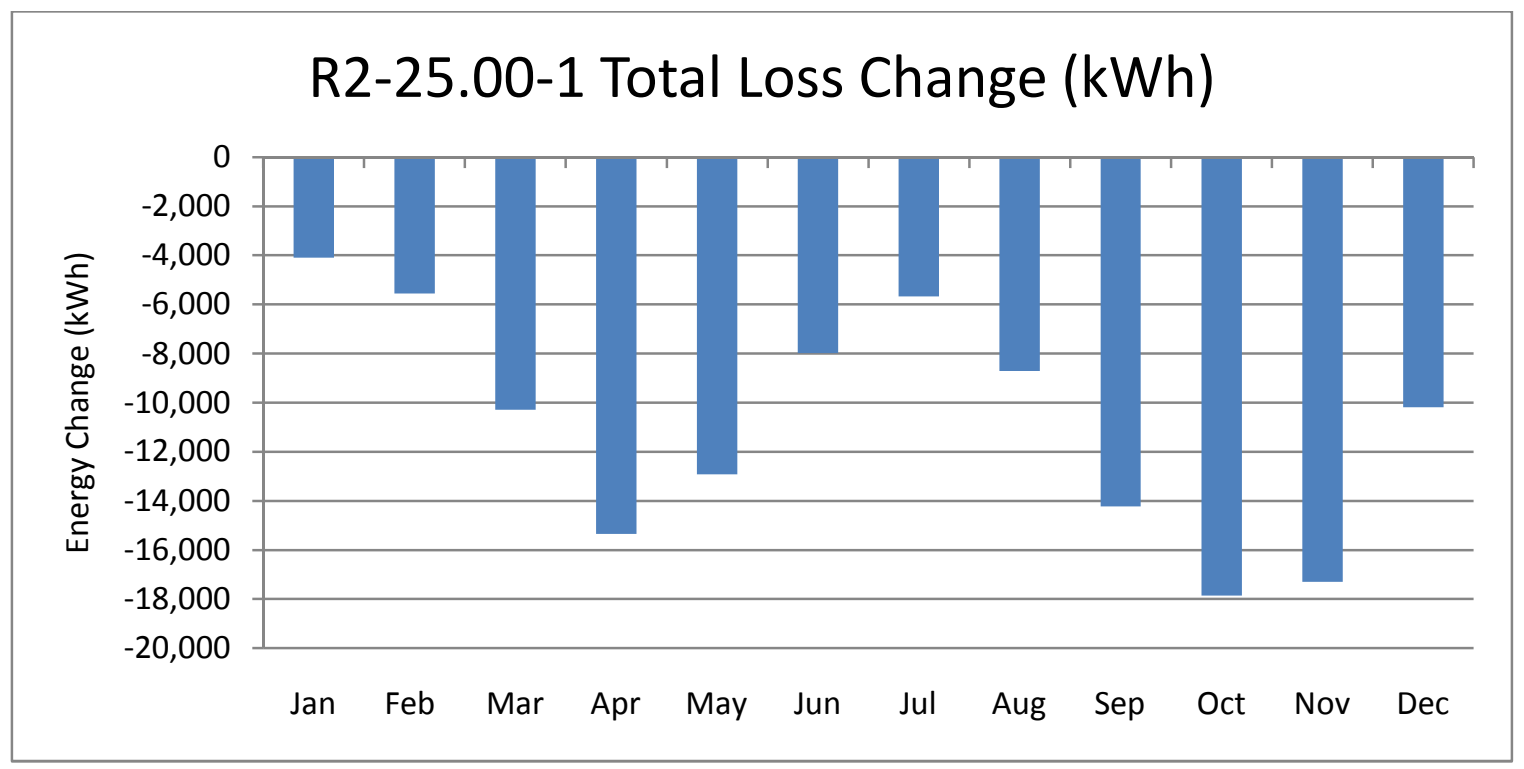

Figure 6.59: R2-25.00-1 Total Loss Change (kWh)

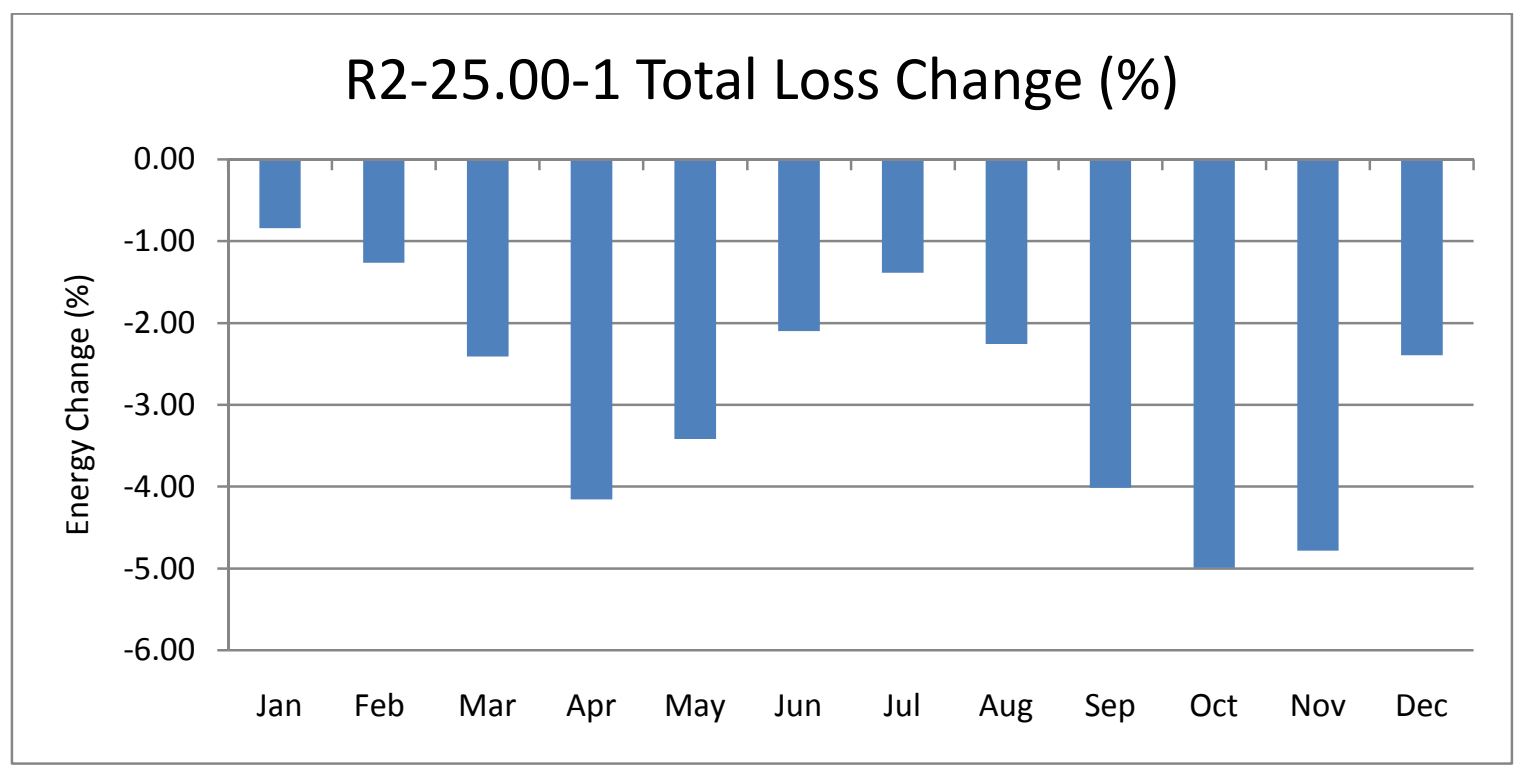

Figure 6.60: R2-25.00-1 Total Loss Change (\%) 


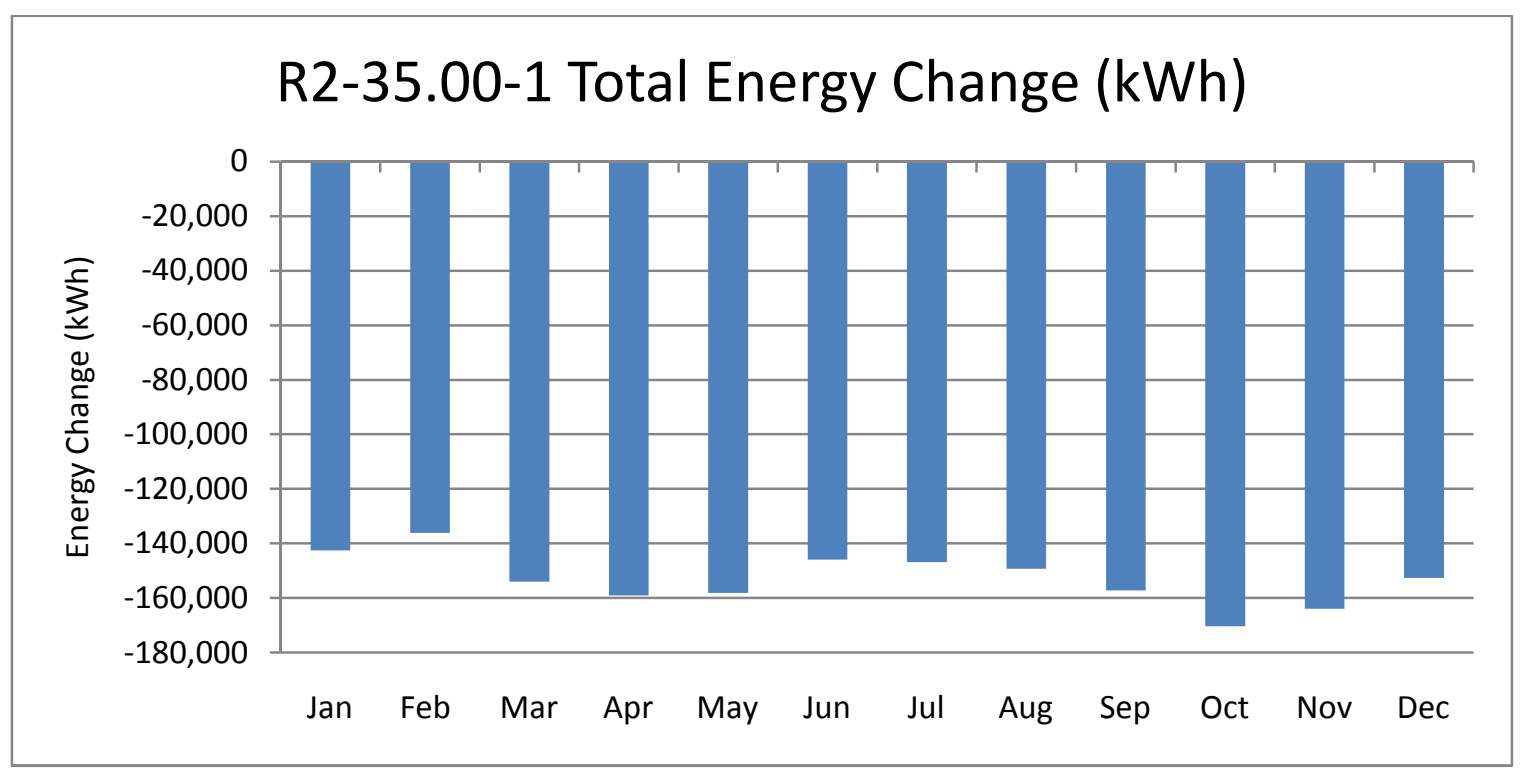

Figure 6.61: R2-35.00-1 Total Energy Change (kWh)

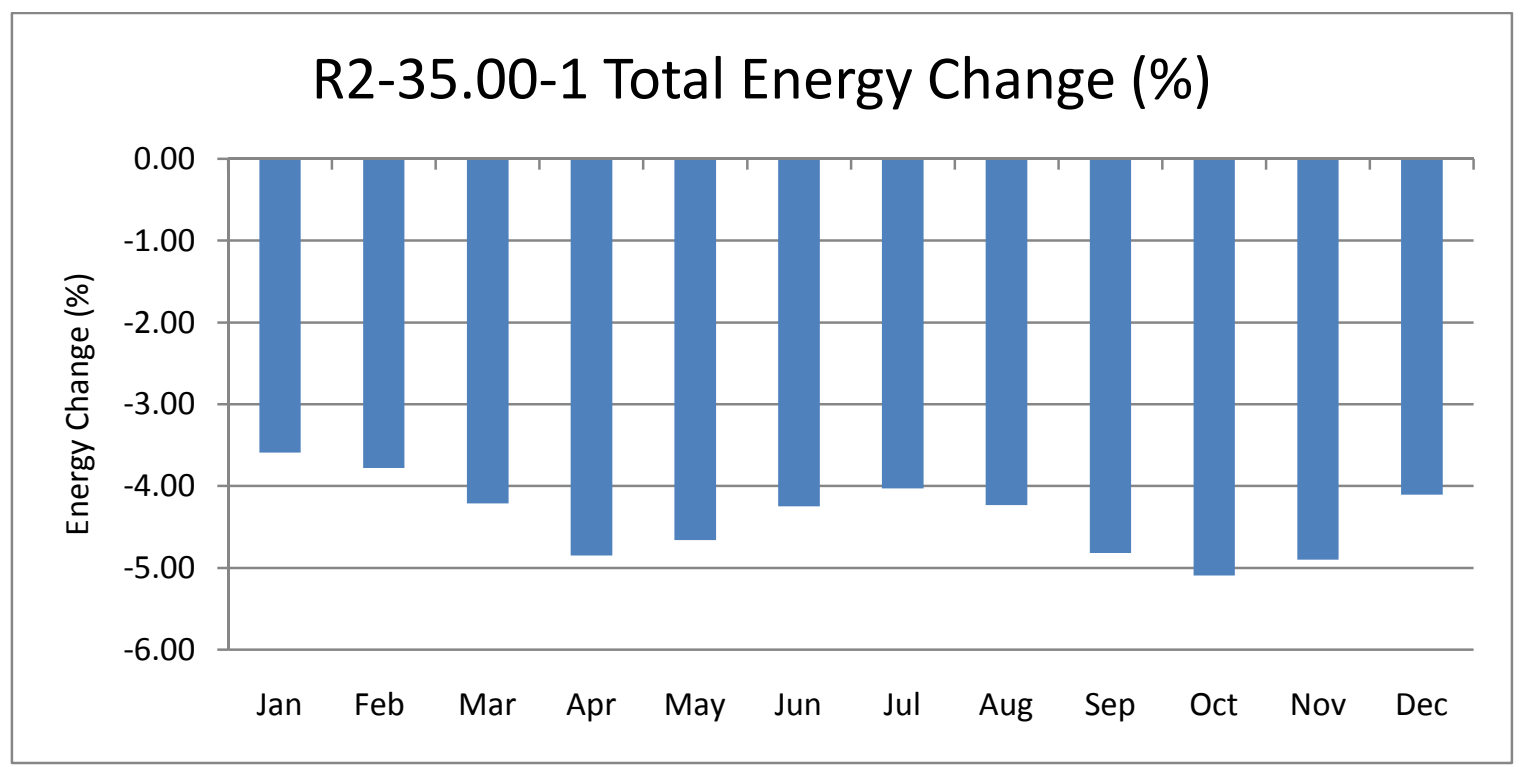

Figure 6.62: R2-35.00-1 Total Energy Change (\%) 


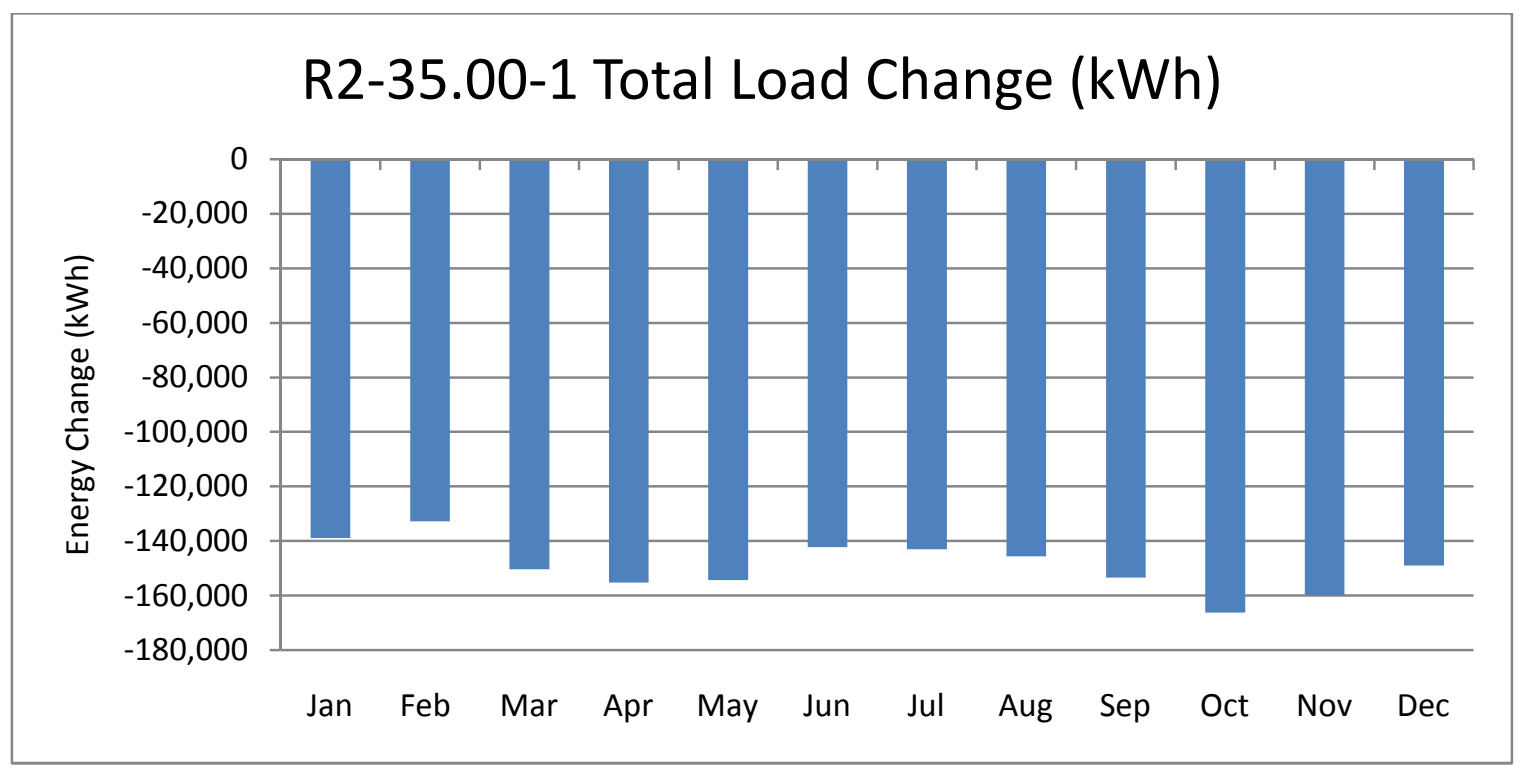

Figure 6.63: R2-35.00-1 Total Load Change (kWh)

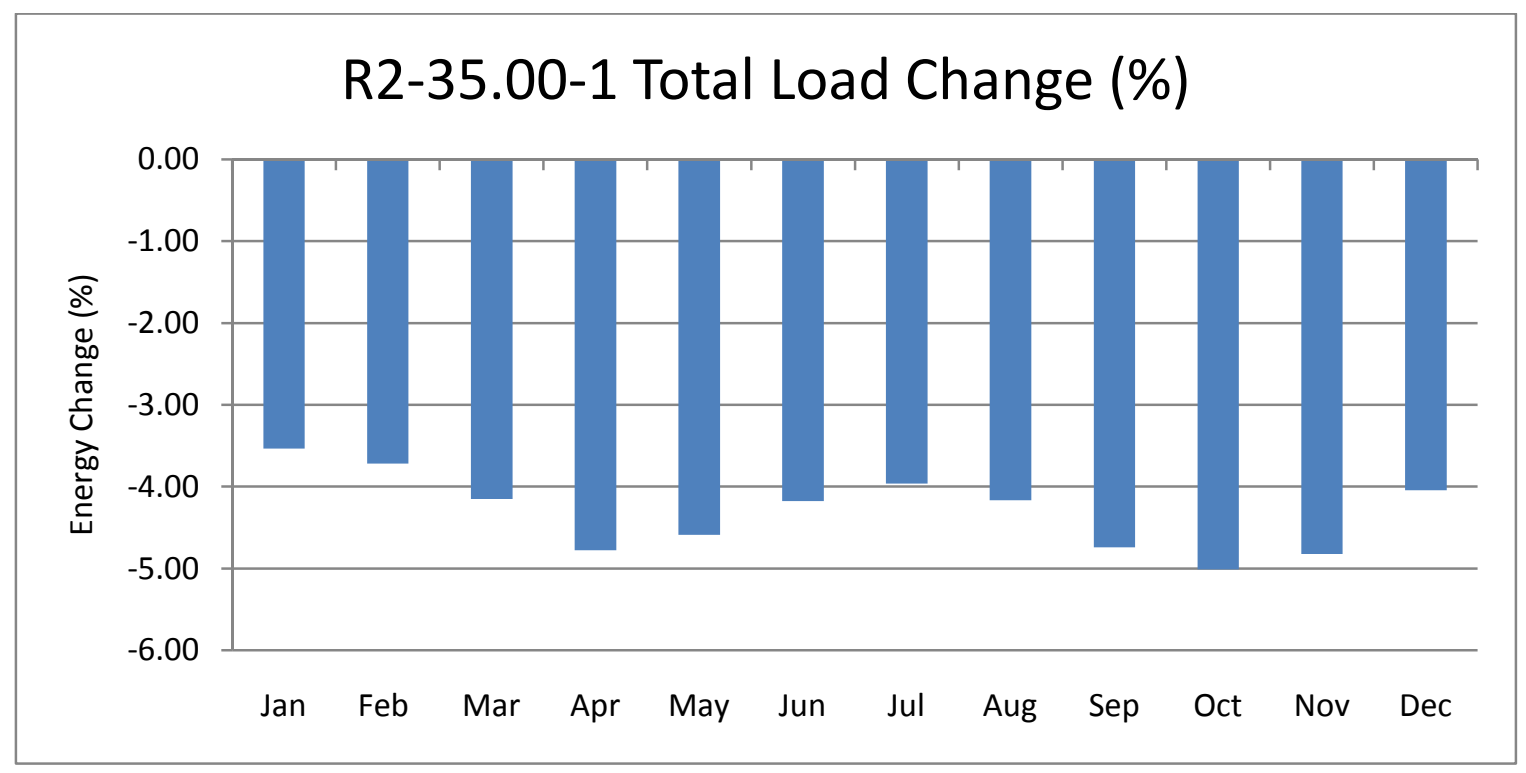

Figure 6.64: R2-35.00-1 Total Load Change (\%) 


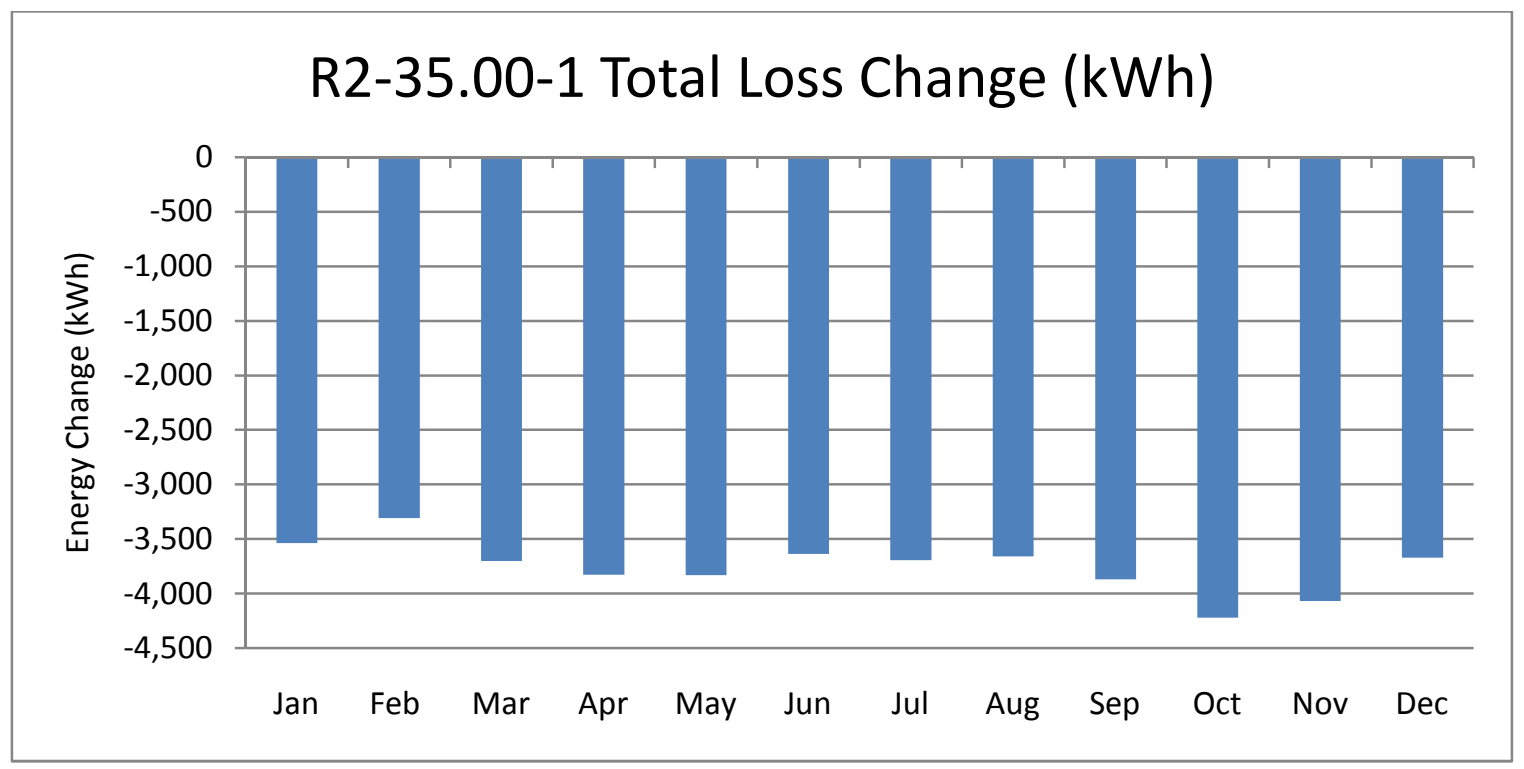

Figure 6.65: R2-35.00-1 Total Loss Change (kWh)

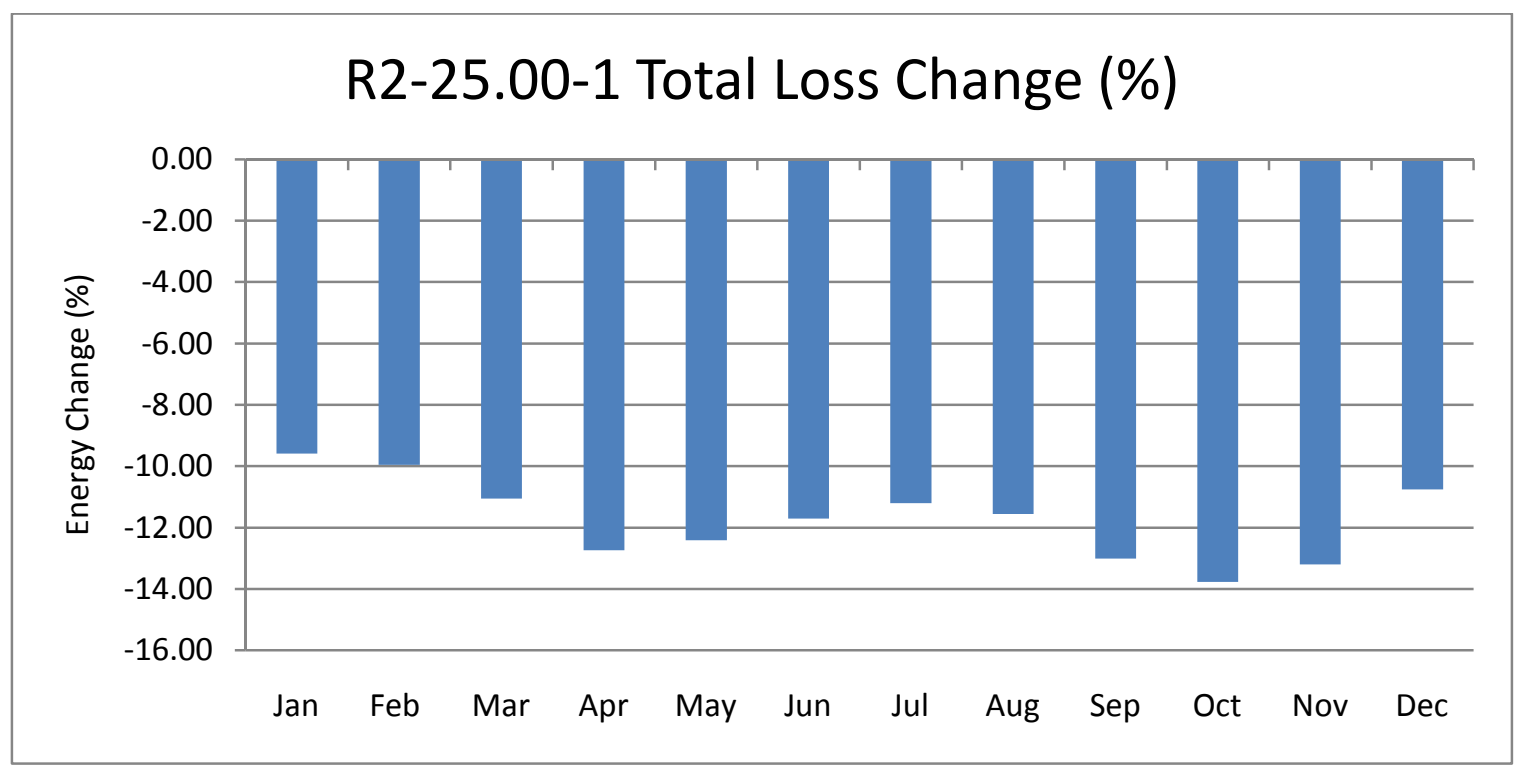

Figure 6.66: R2-35.00-1 Total Loss Change (\%)

\subsection{Region 3: CVR Plots}




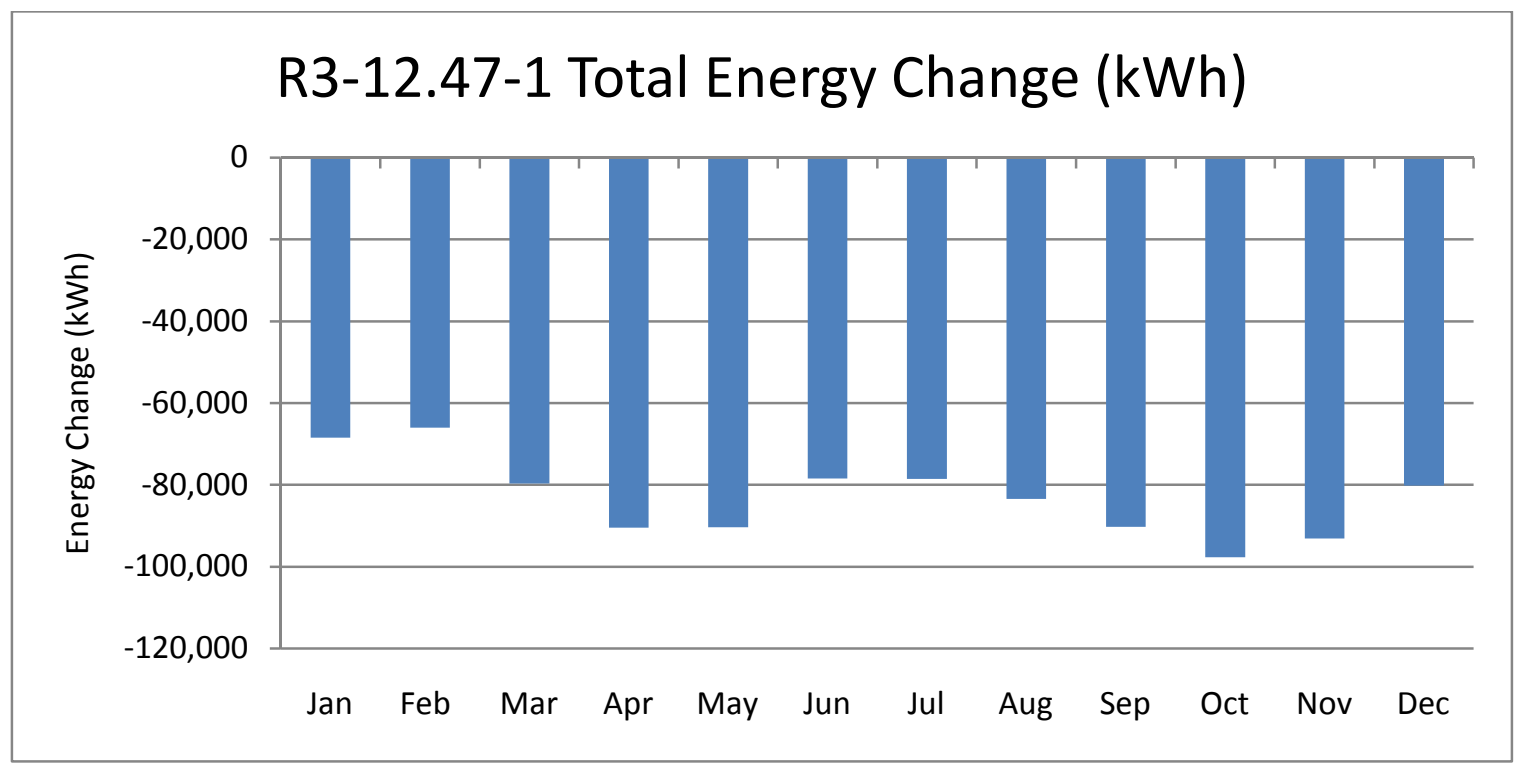

Figure 6.67: R3-12.47-1 Total Energy Change (kWh)

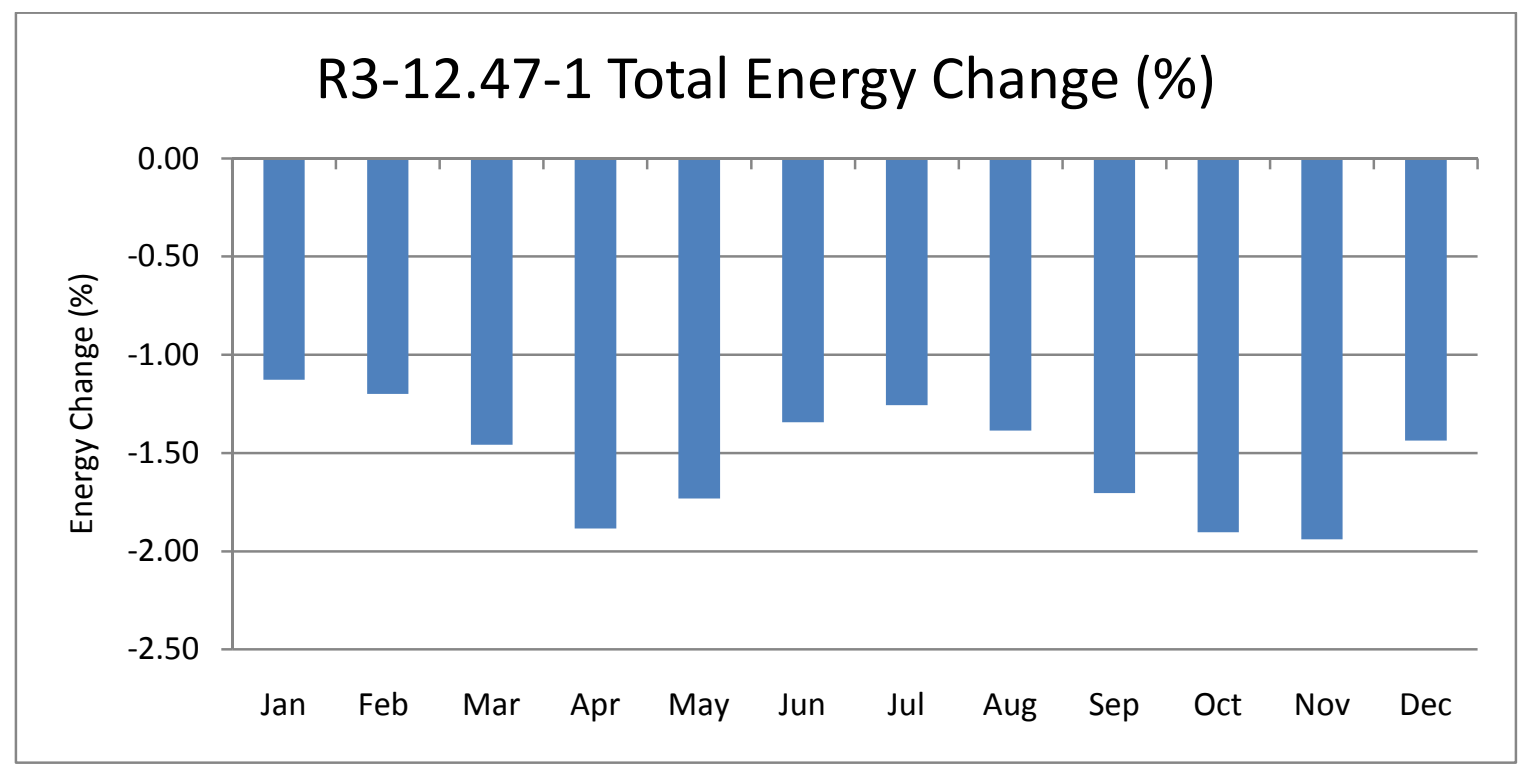

Figure 6.68: R3-12.47-1 Total Energy Change (\%) 


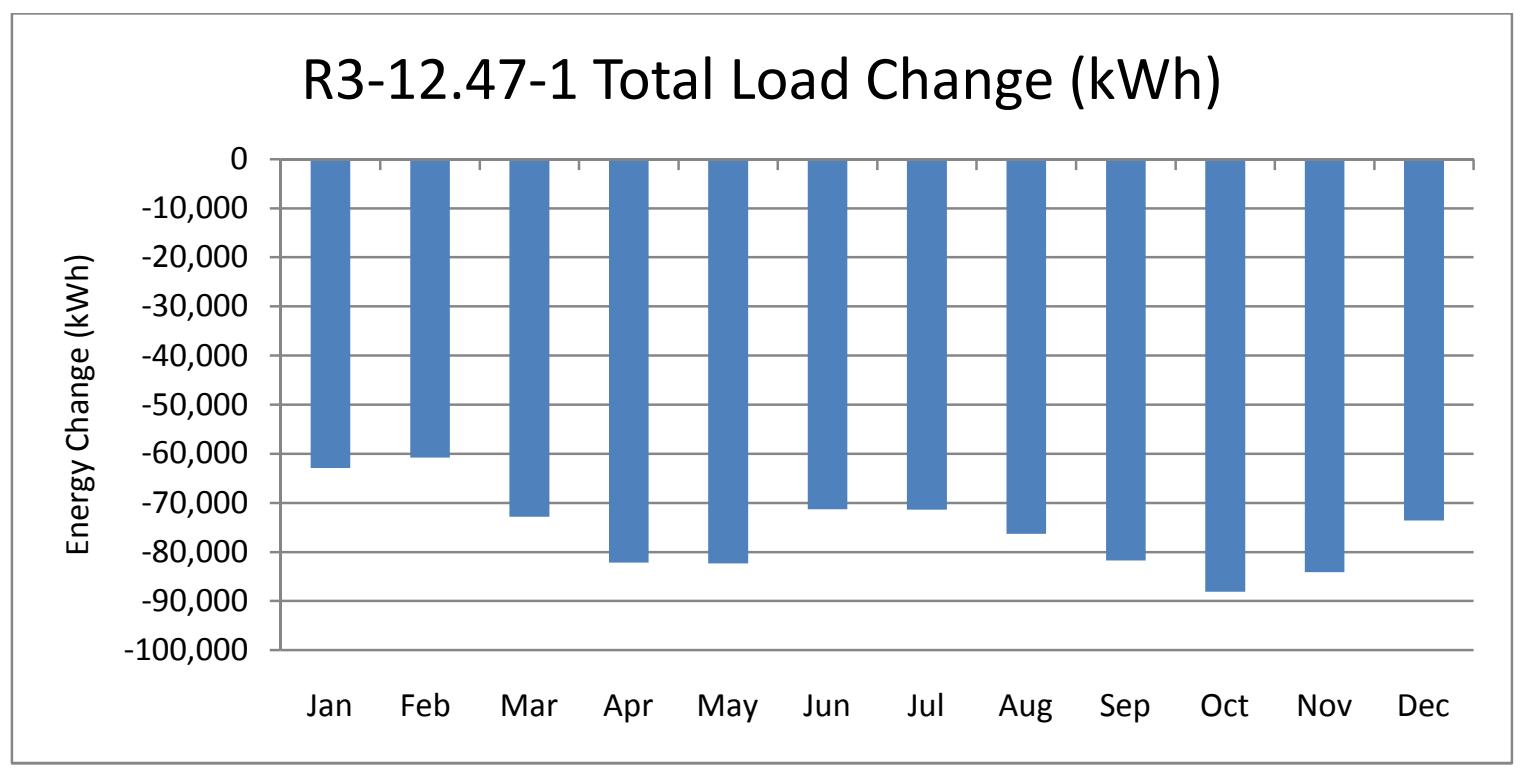

Figure 6.69: R3-12.47-1 Total Loss Change (kWh)

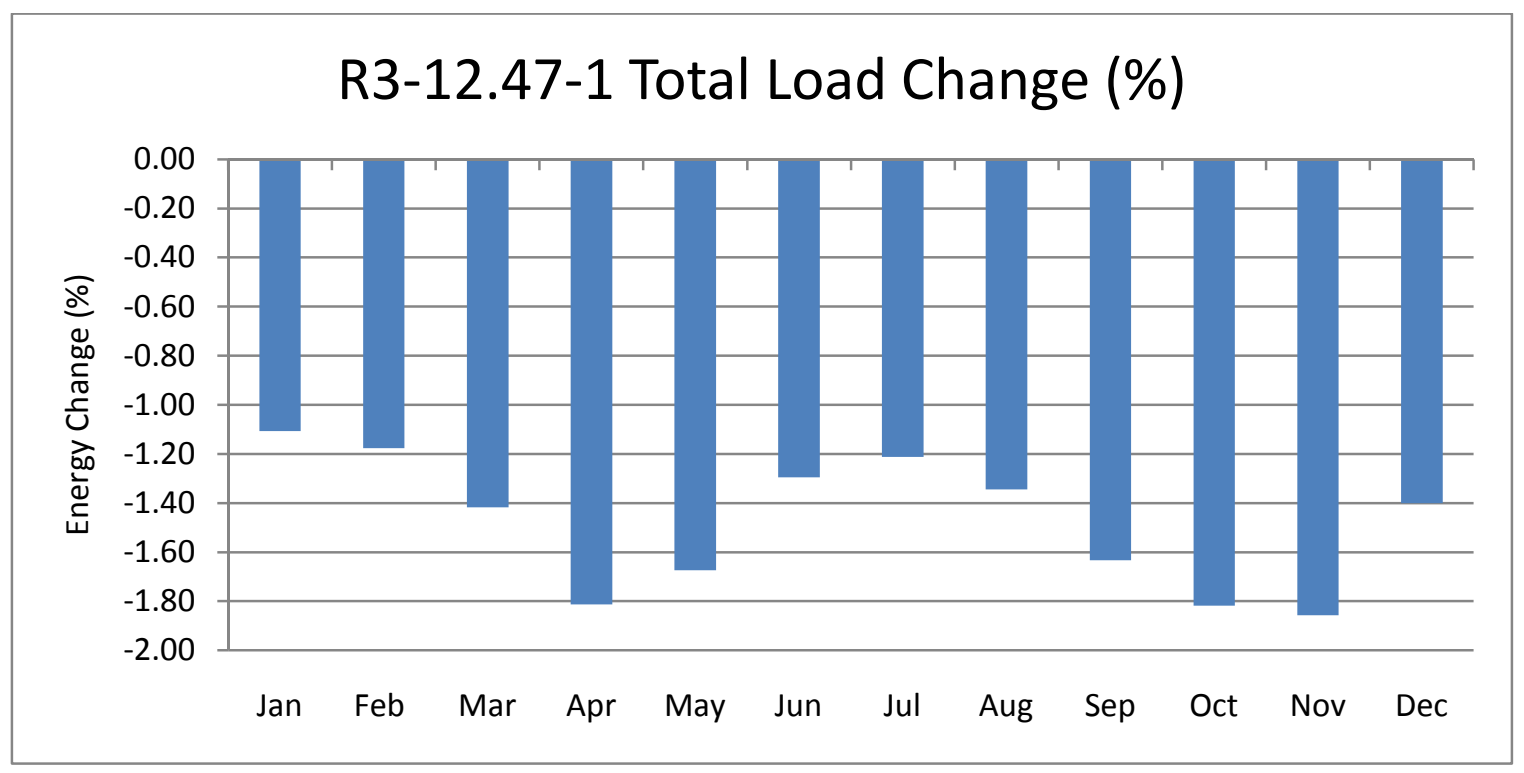

Figure 6.70: R3-12.47-1 Total Loss Change (\%) 


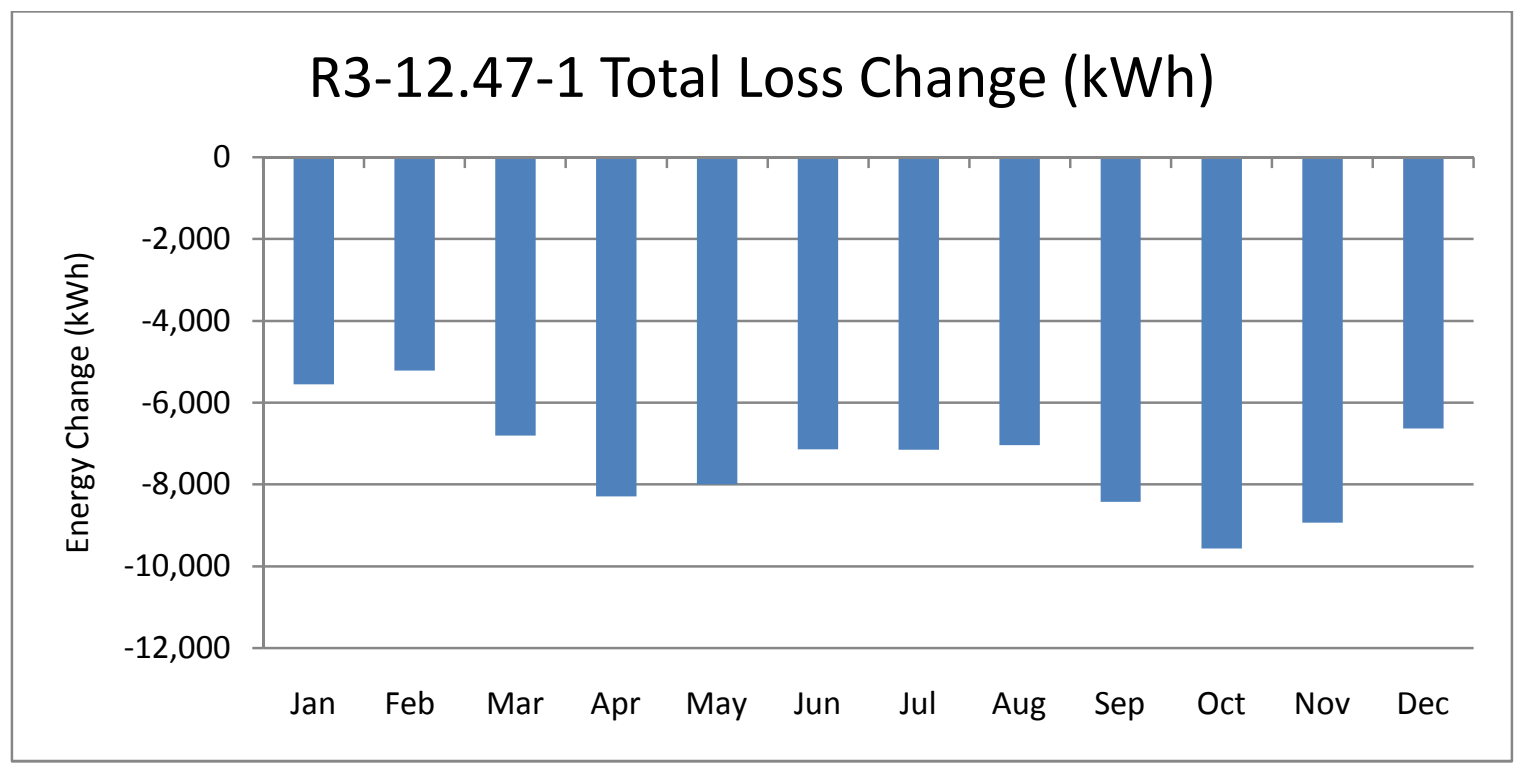

Figure 6.71: R3-12.47-1 Total Loss Change (kWh)

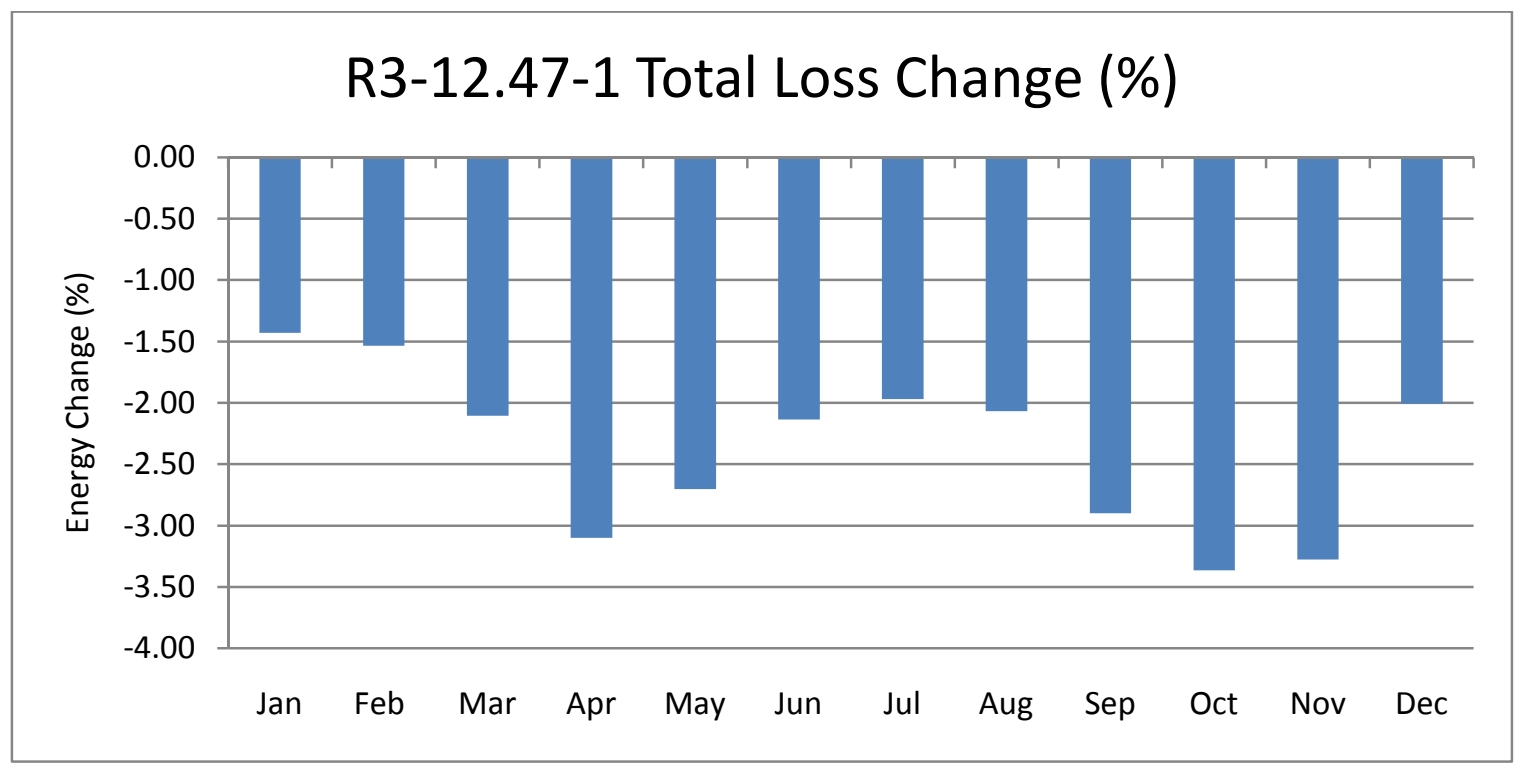

Figure 6.72: R3-12.47-1 Total Loss Change (\%) 


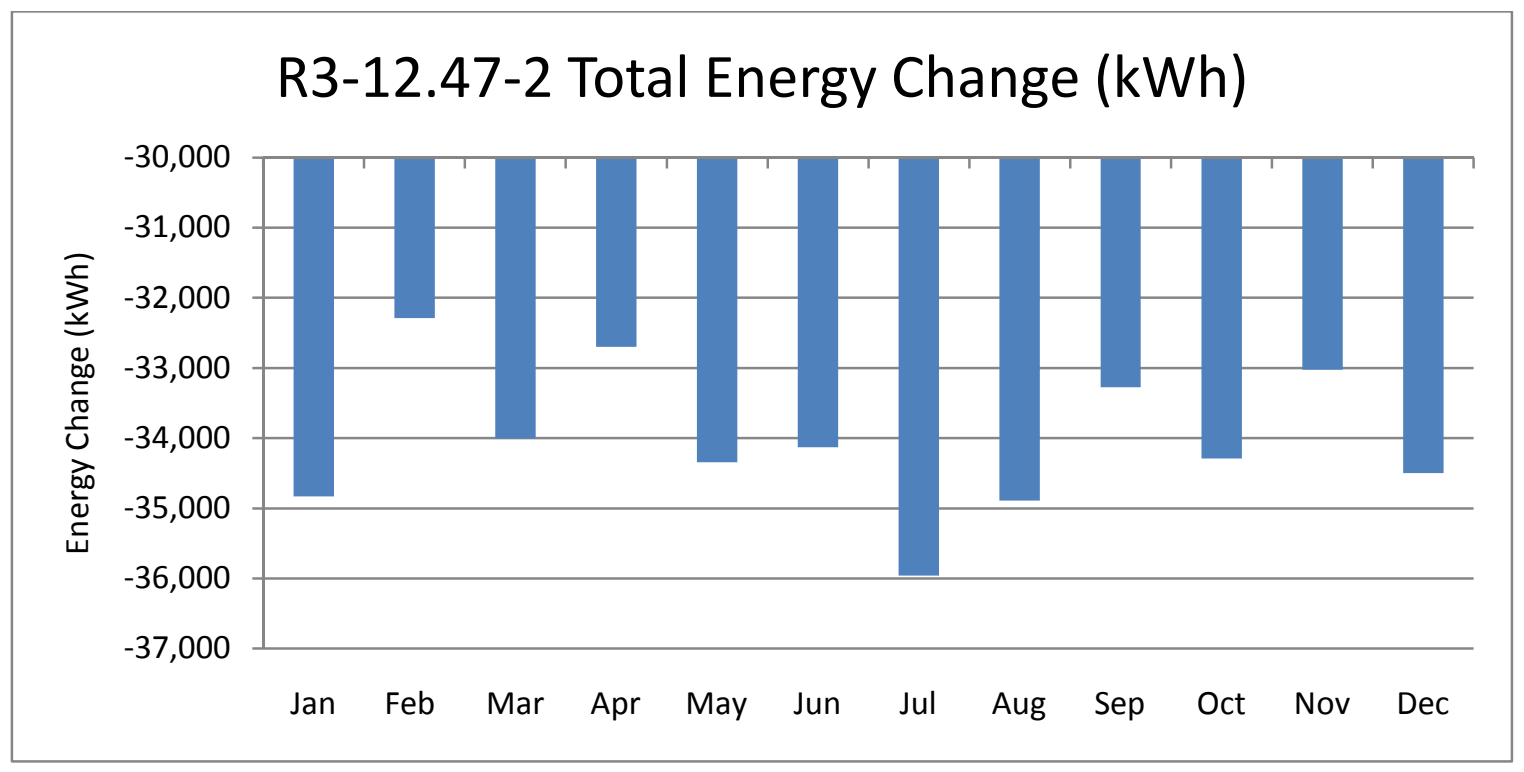

Figure 6.73: R3-12.47-2 Total Energy Change (kWh)

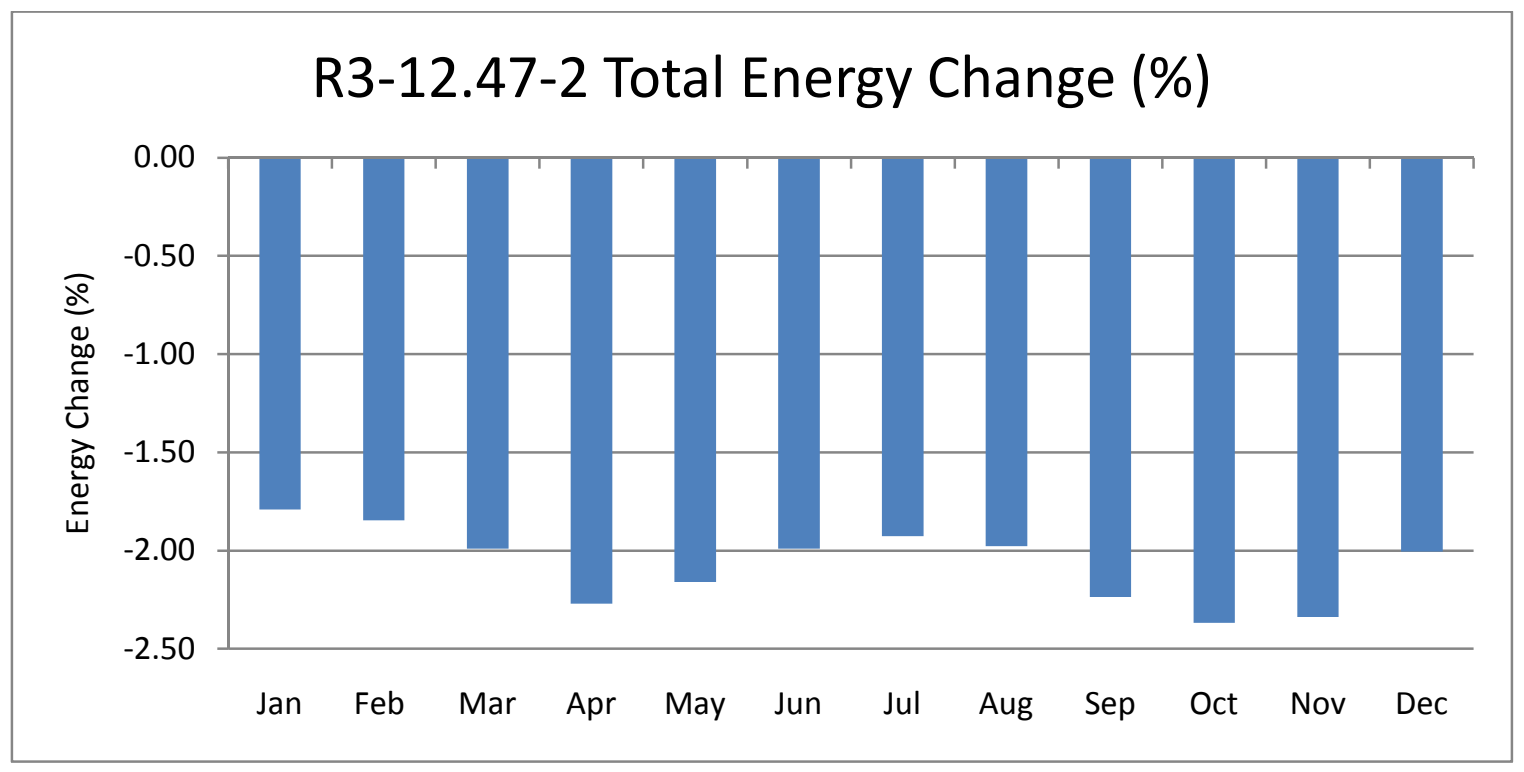

Figure 6.74: R3-12.47-2 Total Energy Change (\%) 


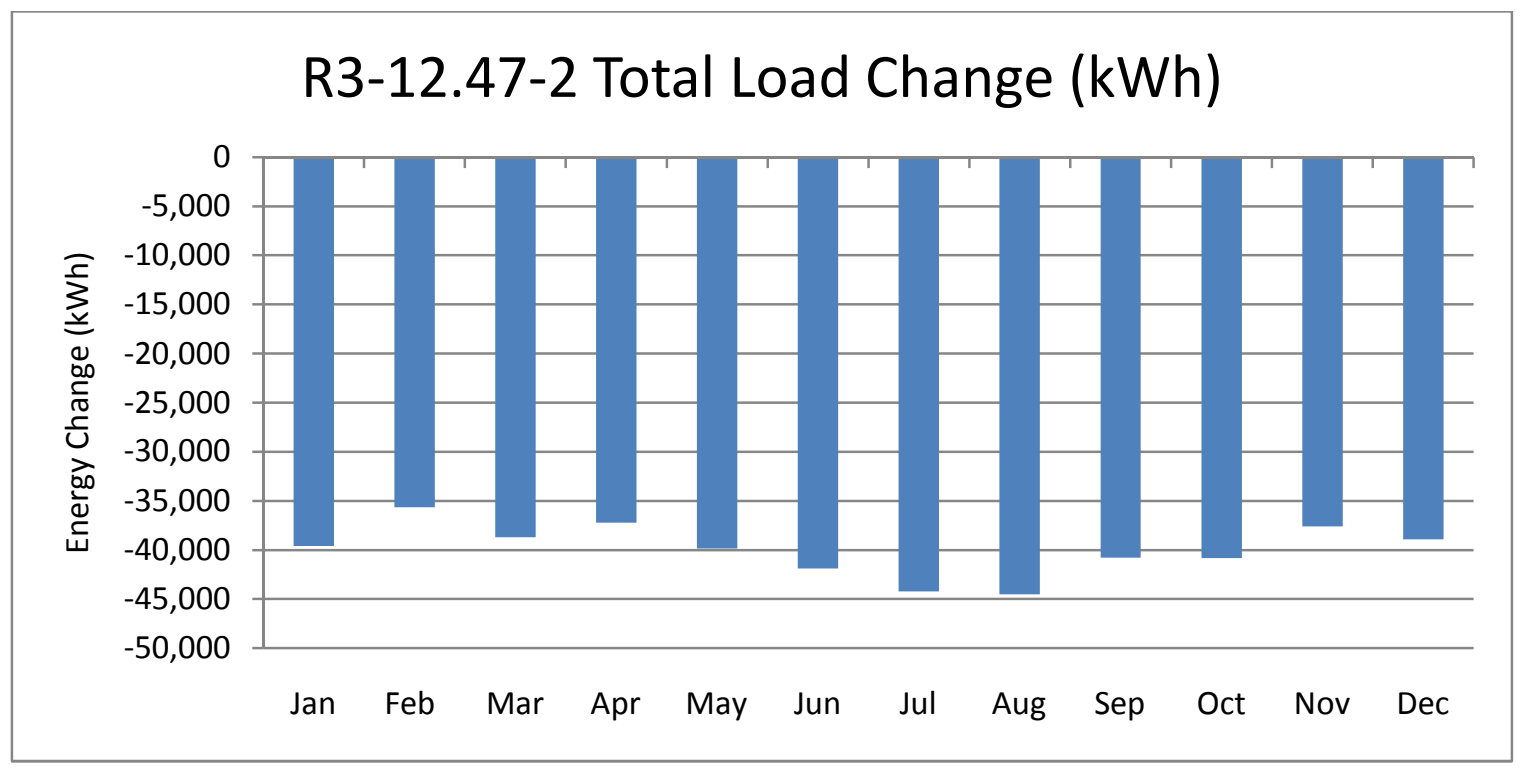

Figure 6.75: R3-12.47-2 Total Load Change (kWh)

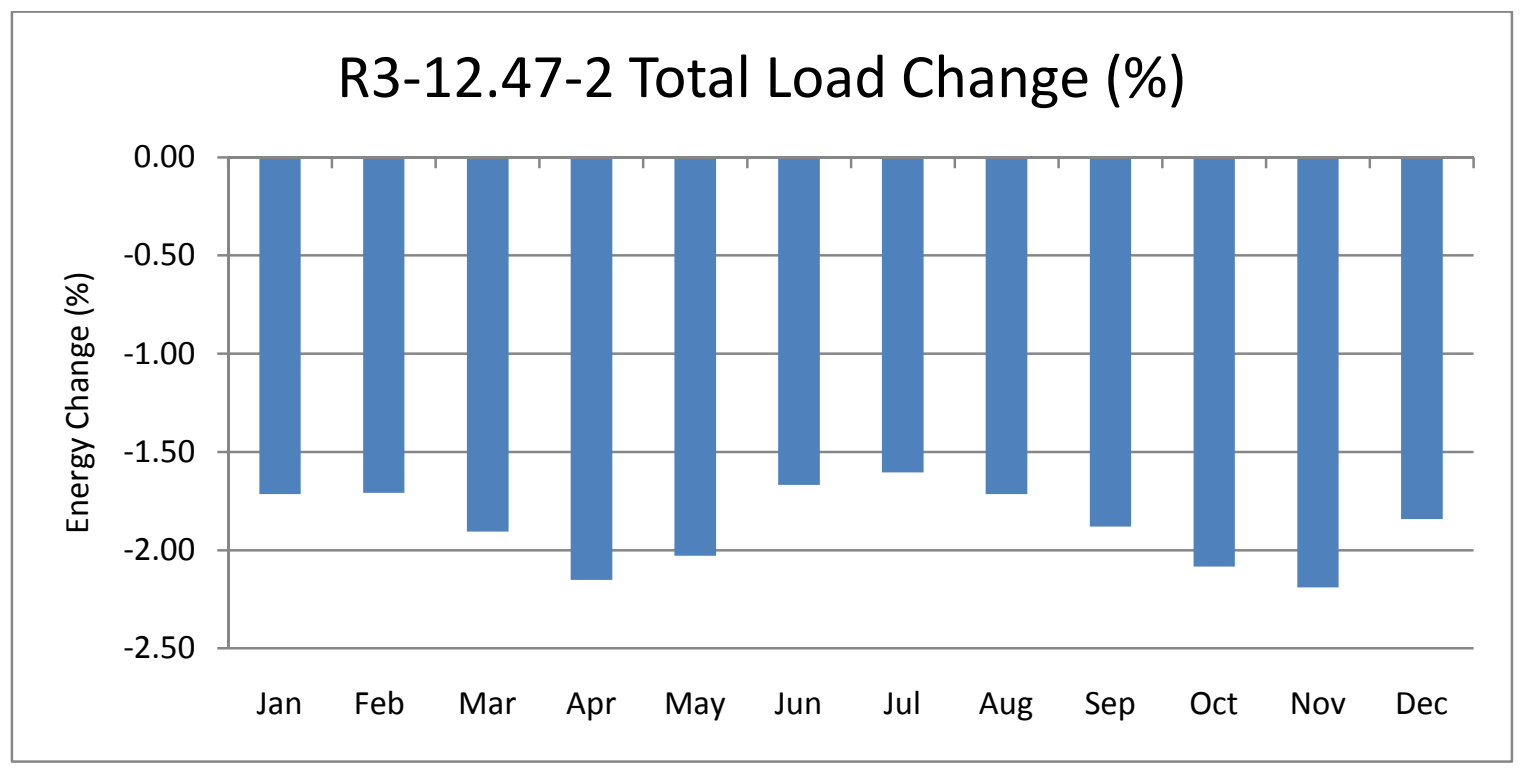

Figure 6.76: R3-12.47-2 Total Load Change (\%) 


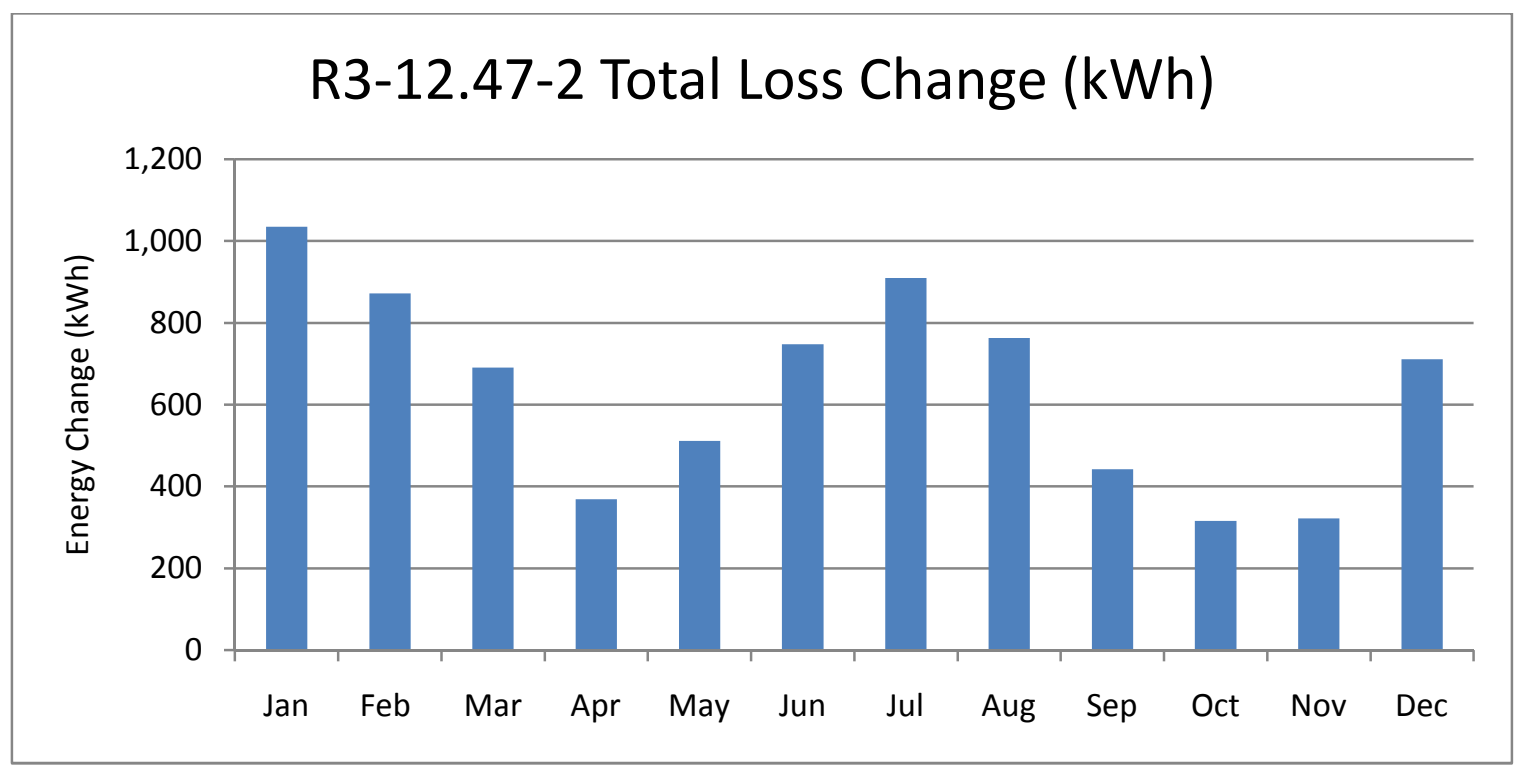

Figure 6.77: R3-12.47-2 Total Loss Change (kWh)

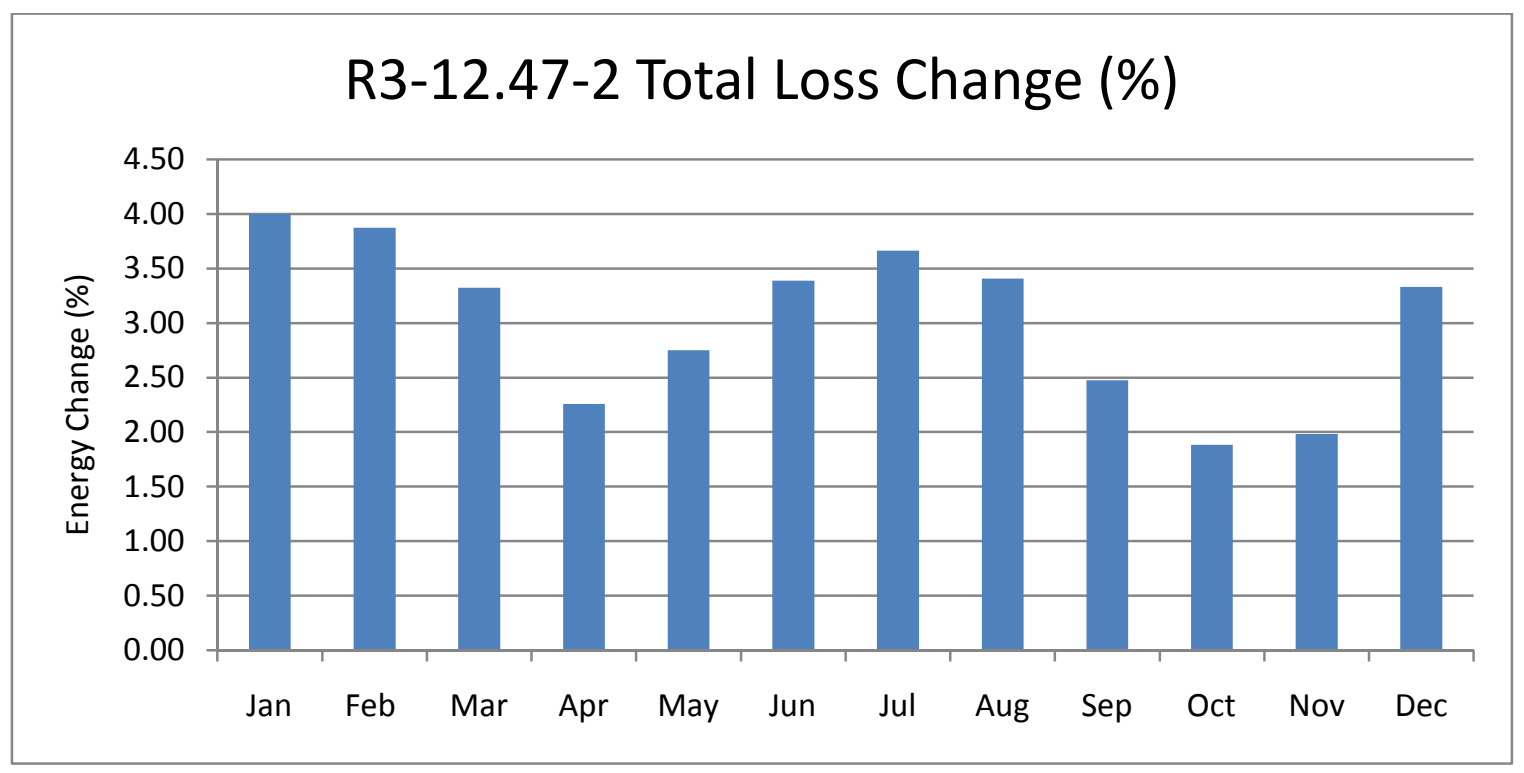

Figure 6.78: R3-12.47-2 Total Loss Change (\%) 


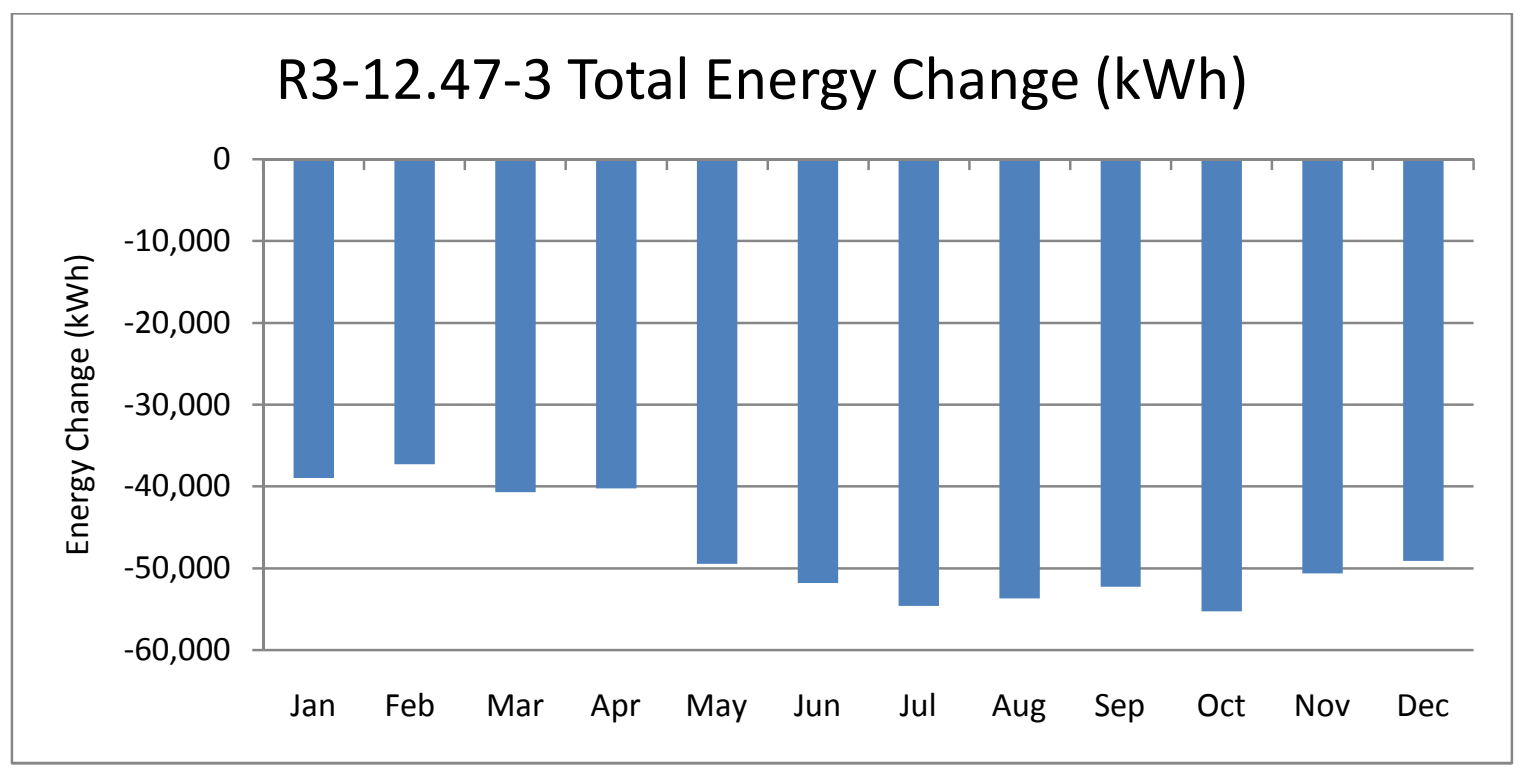

Figure 6.79: R3-12.47-3 Total Energy Change (kWh)

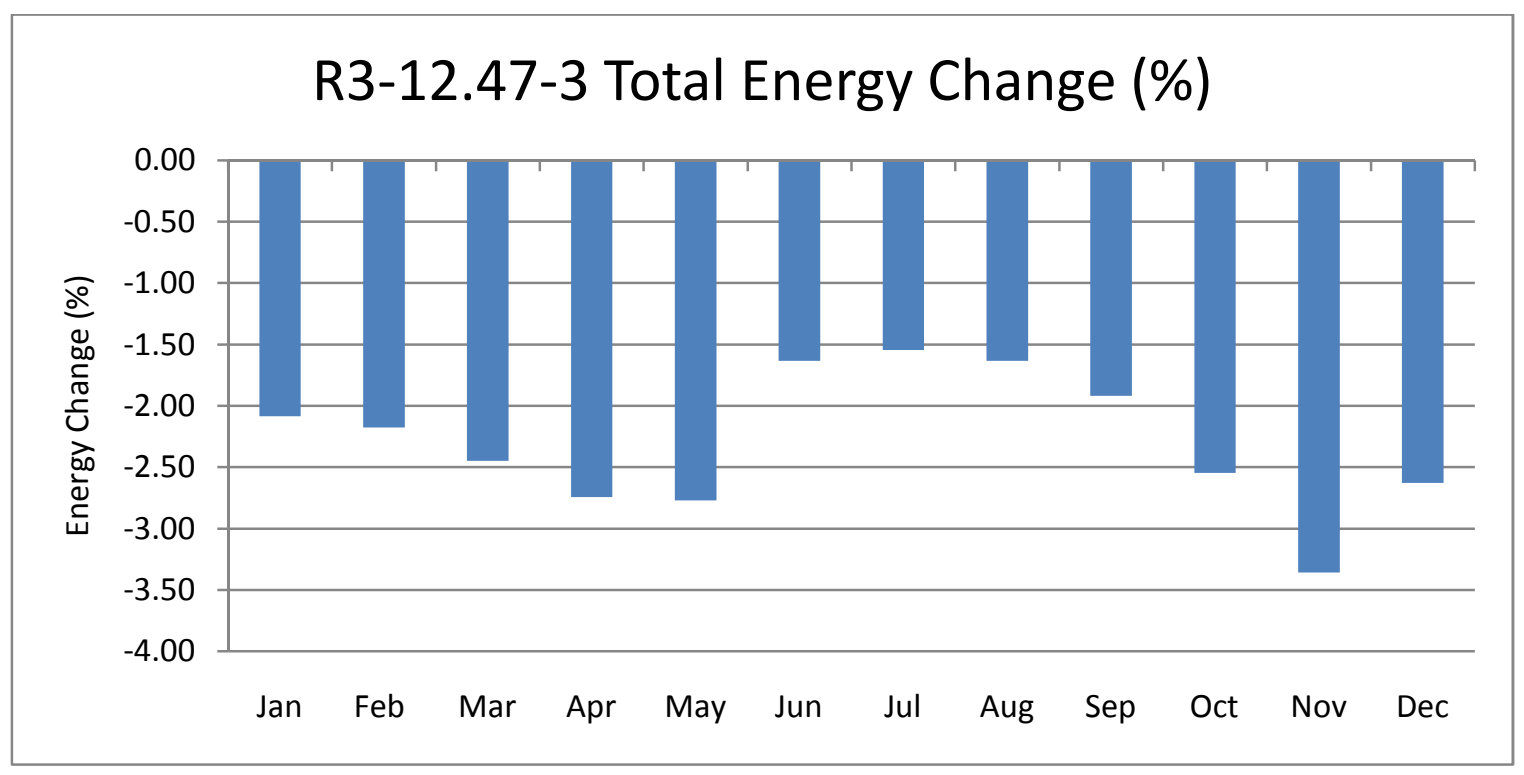

Figure 6.80: R3-12.47-3 Total Energy Change (\%) 


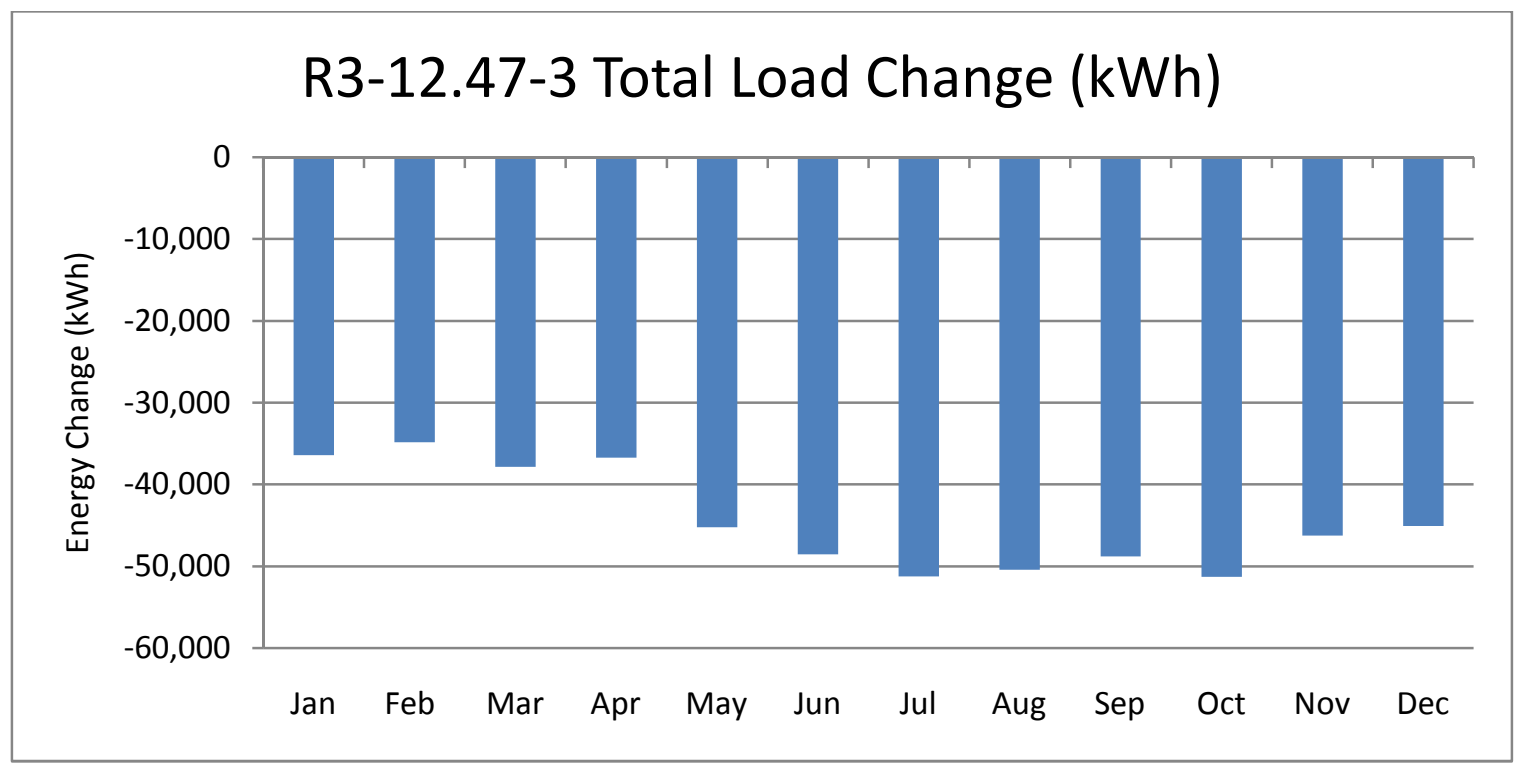

Figure 6.81: R3-12.47-3 Total Load Change (kWh)

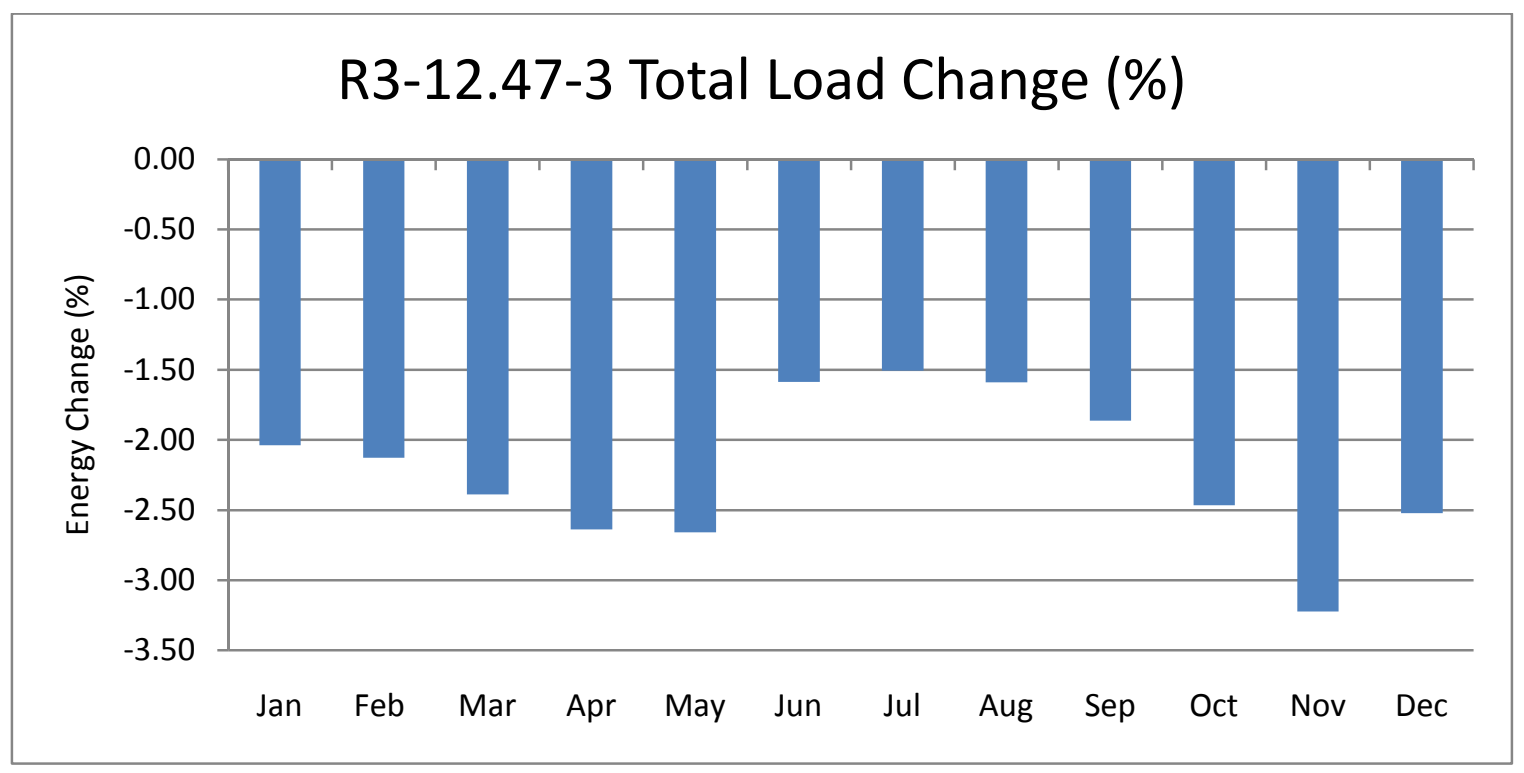

Figure 6.82: R3-12.47-3 Total Load Change (\%) 


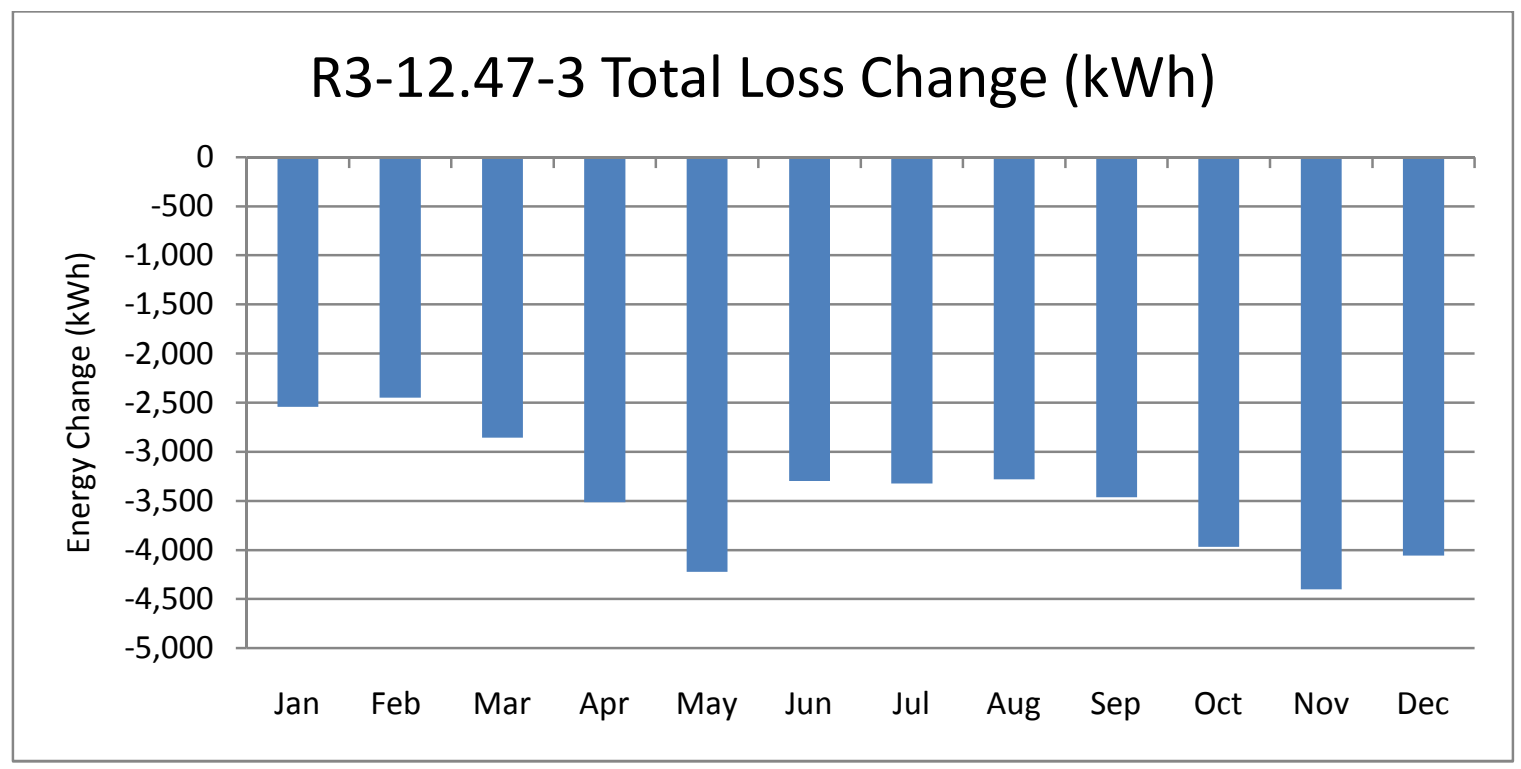

Figure 6.83: R3-12.47-3 Total Loss Change (kWh)

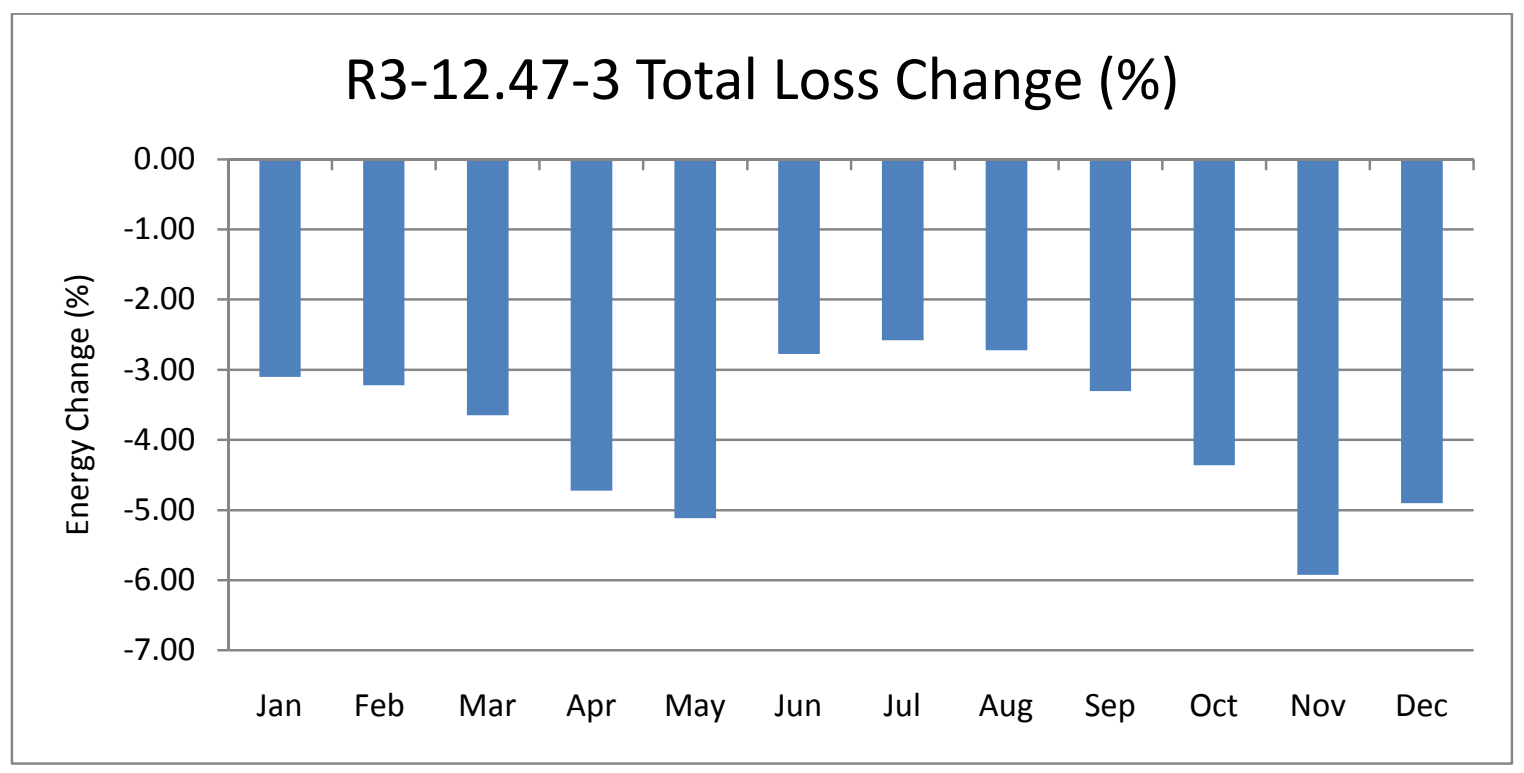

Figure 6.84: R3-12.47-3 Total Loss Change (\%)

\subsection{Region 4: CVR Plots}




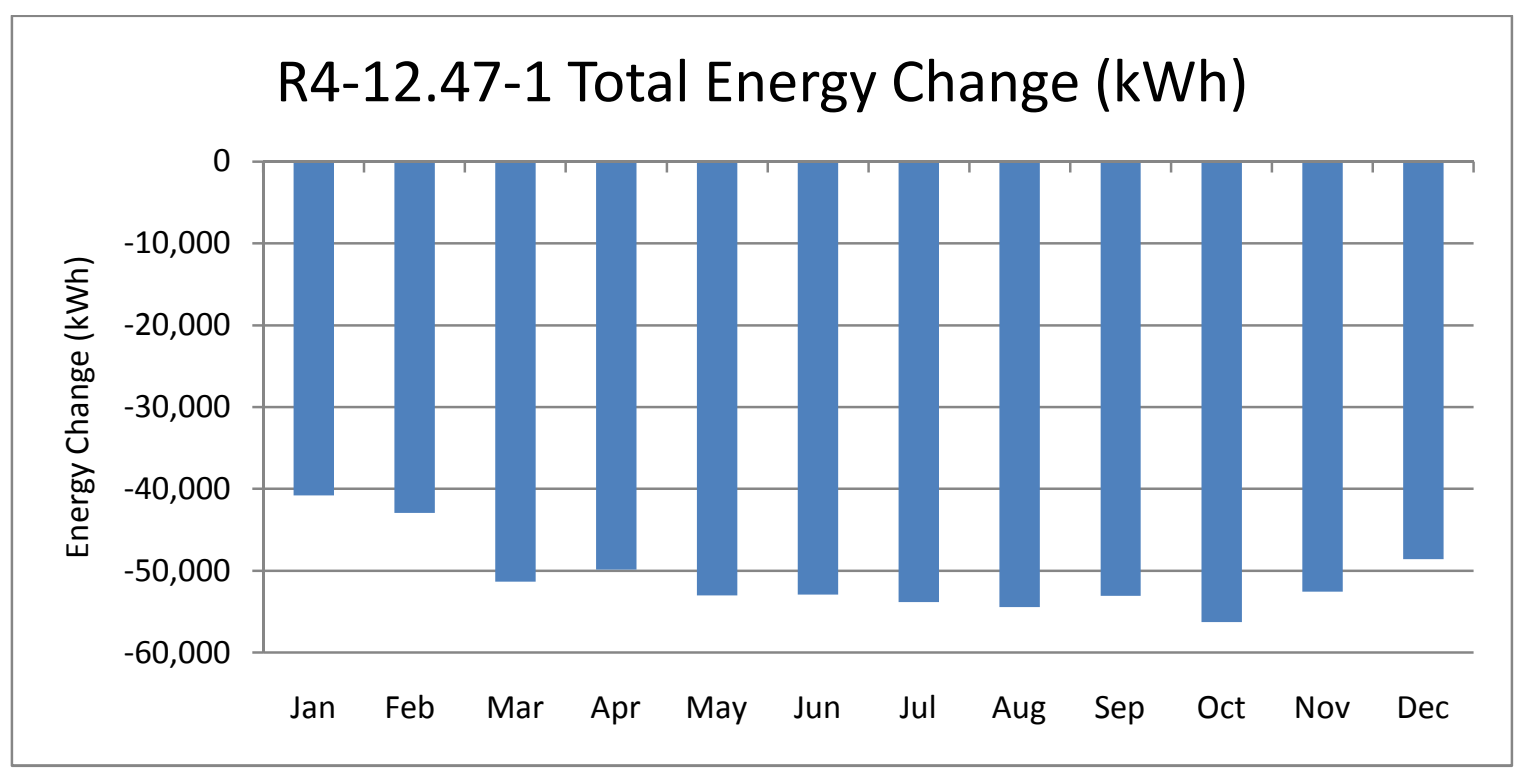

Figure 6.85: R4-12.47-1 Total Energy Change (kWh)

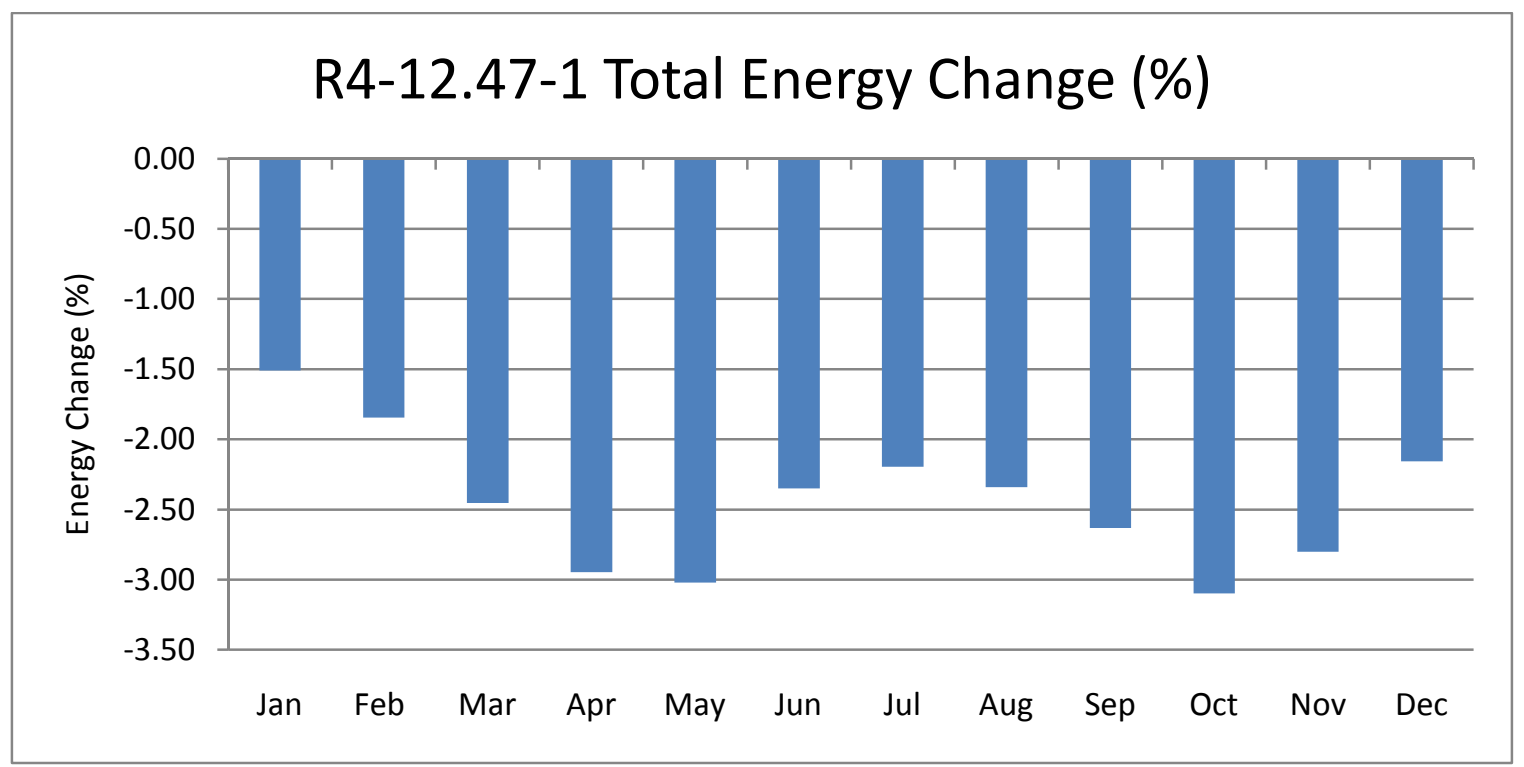

Figure 6.86: R4-12.47-1 Total Energy Change (\%) 


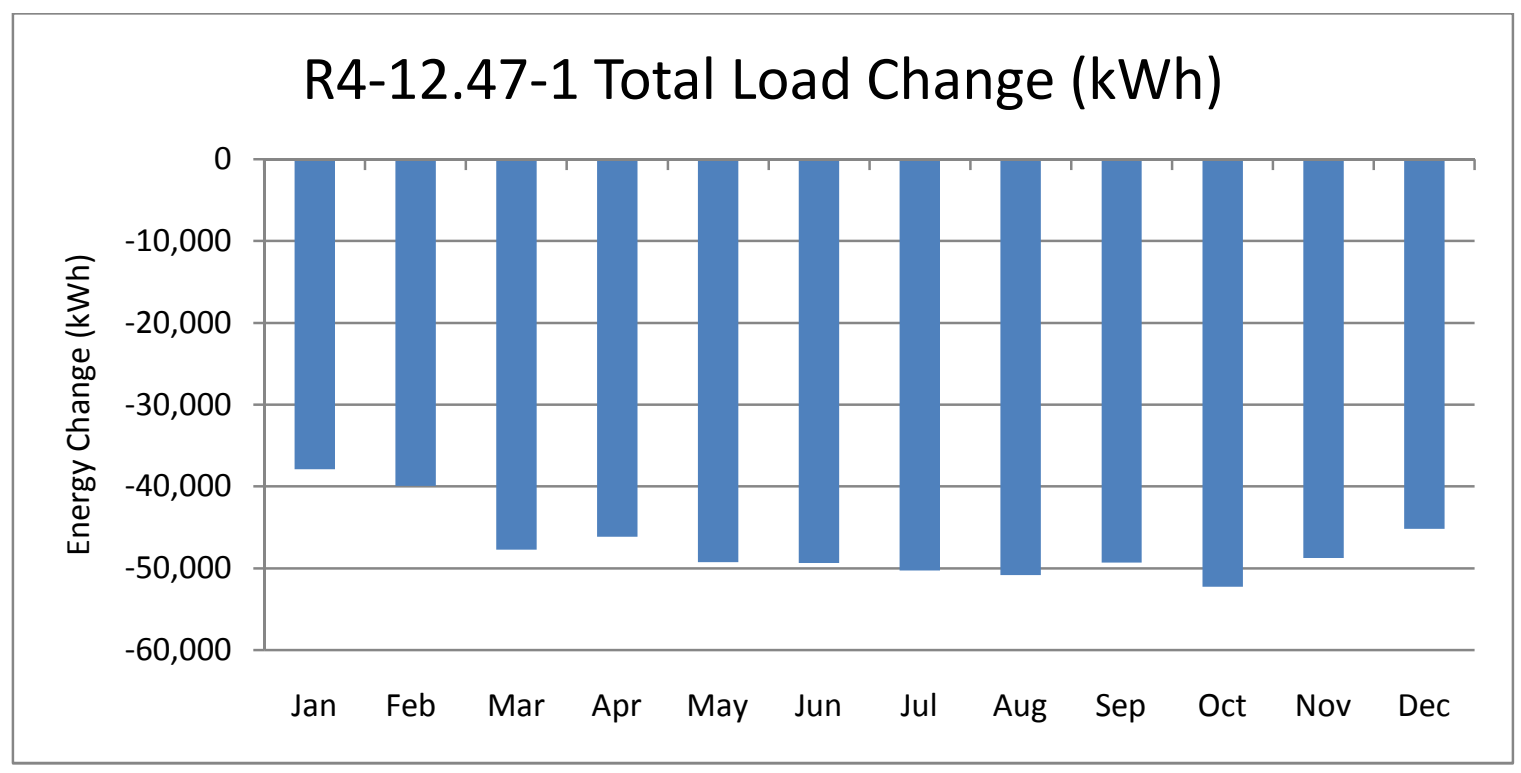

Figure 6.87: R4-12.47-1 Total Load Change (kWh)

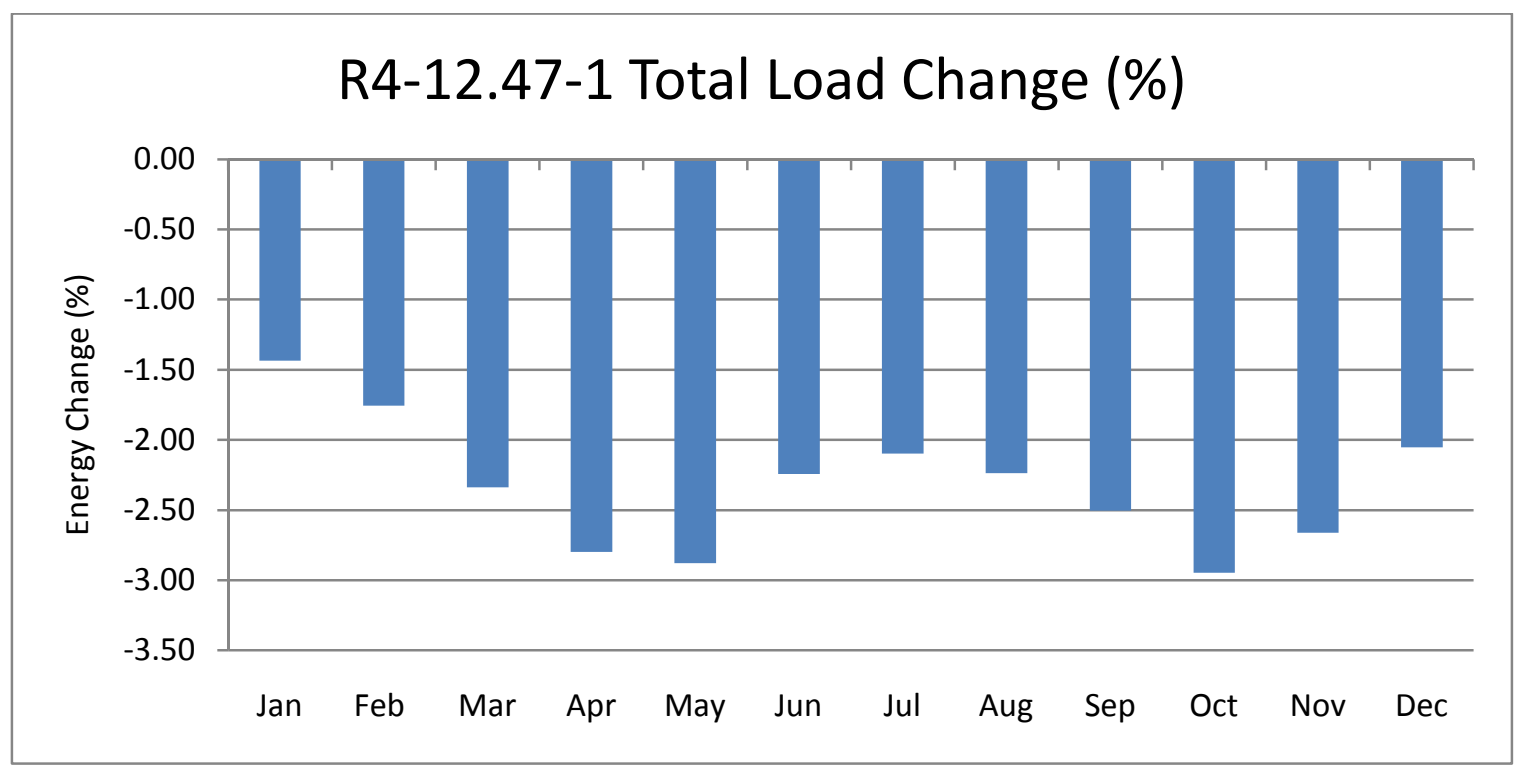

Figure 6.88: R4-12.47-1 Total Load Change (\%) 


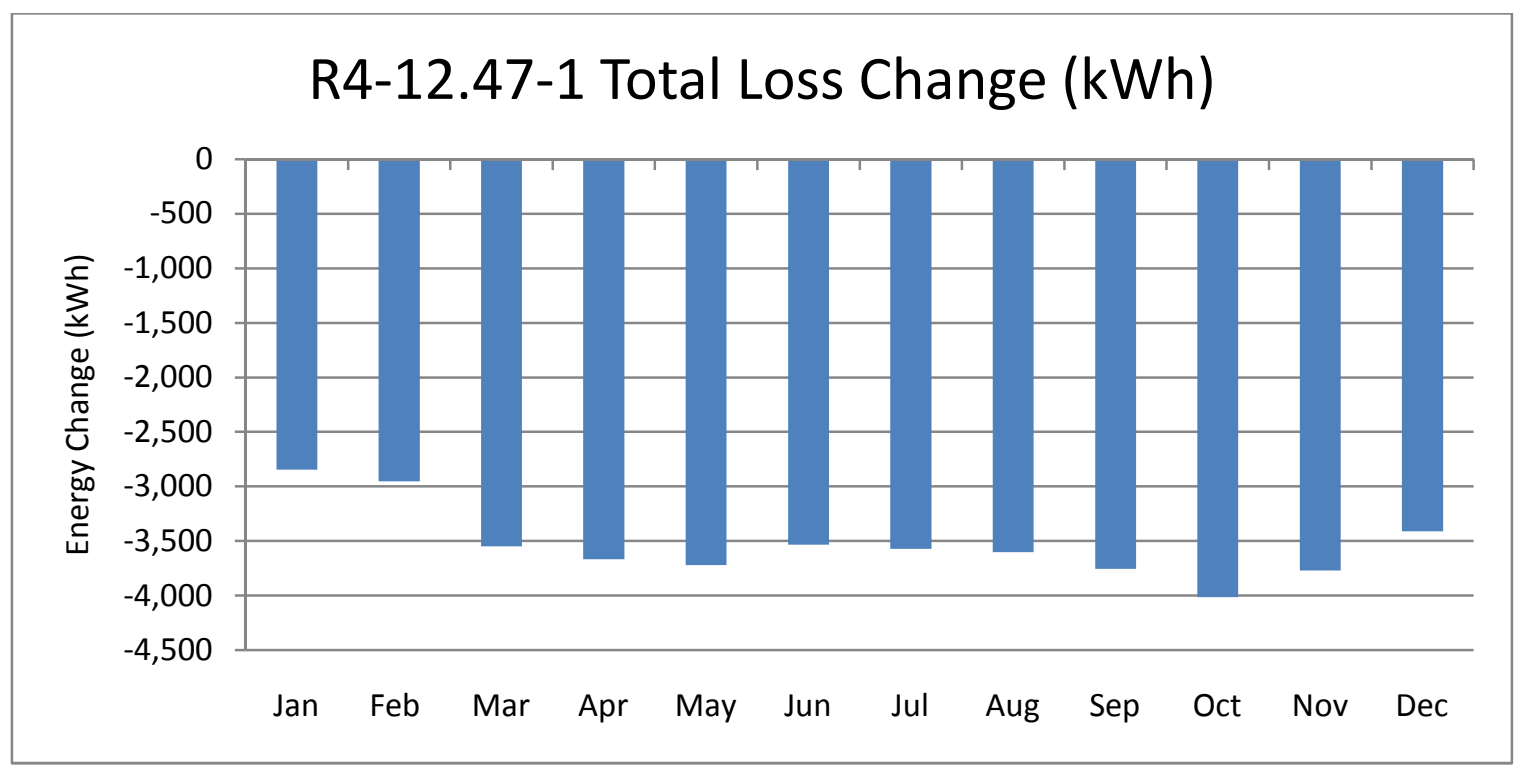

Figure 6.89: R4-12.47-1 Total Loss Change (kWh)

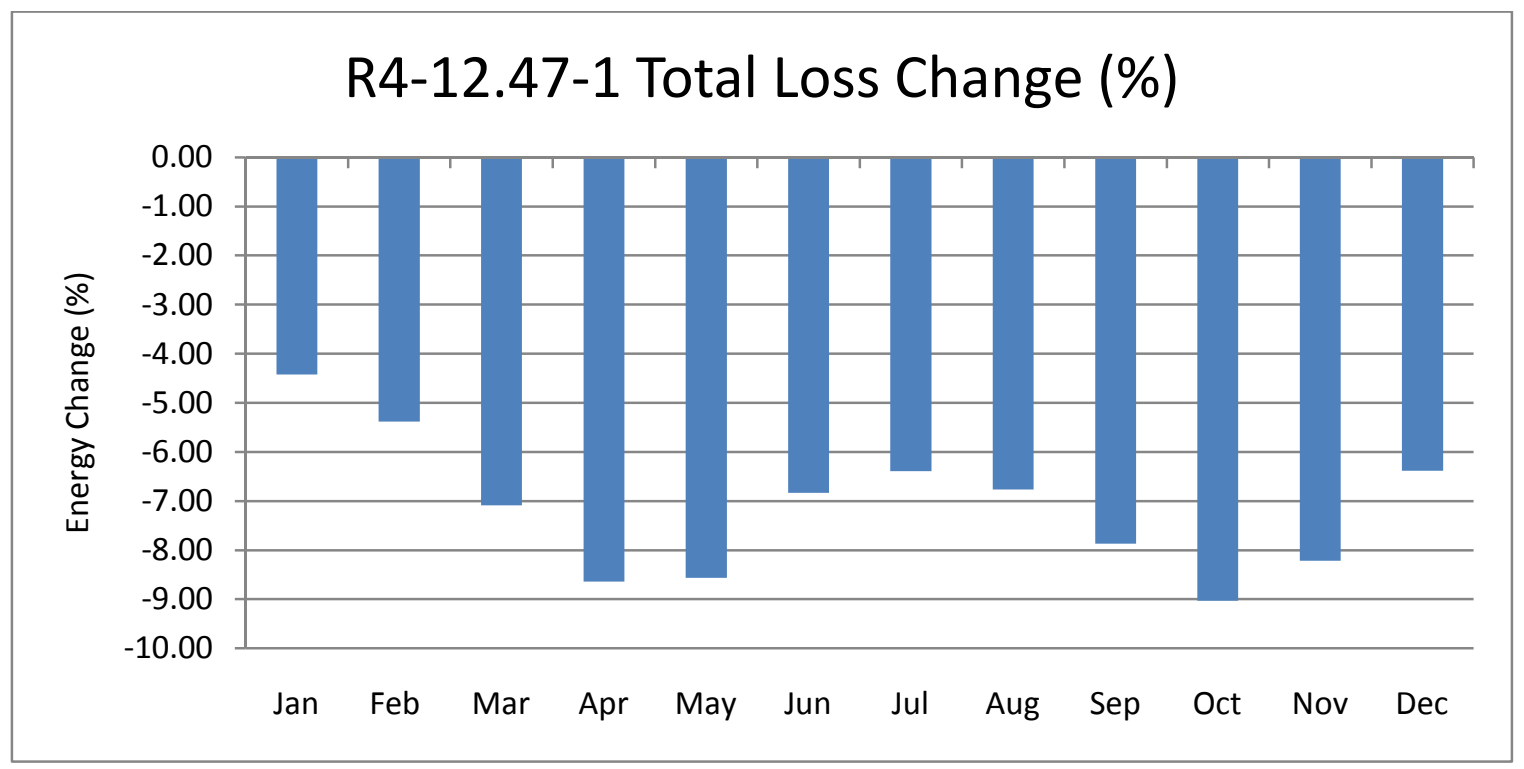

Figure 6.90: R4-12.47-1 Total Loss Change (\%) 


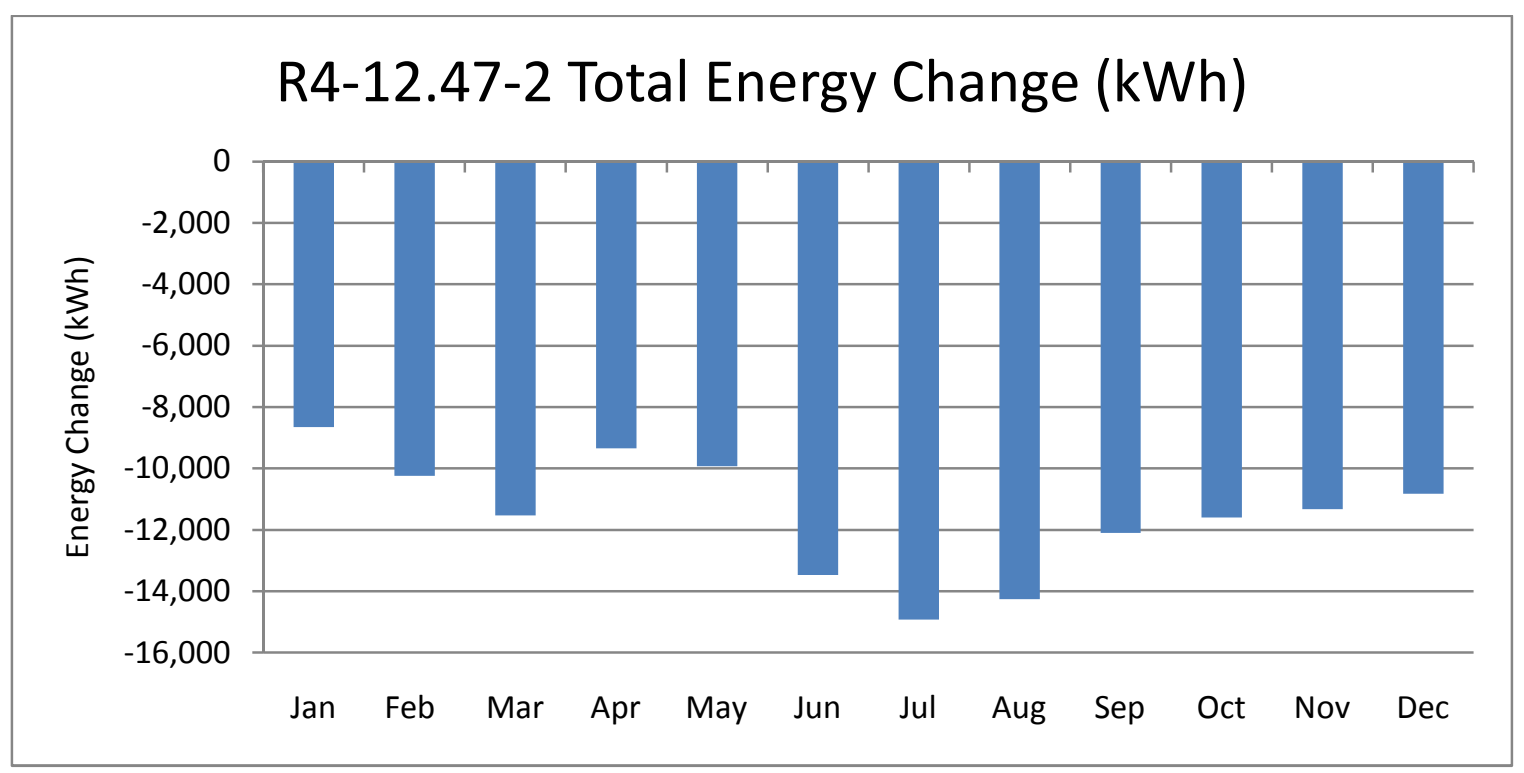

Figure 6.91: R4-12.47-2 Total Energy Change (kWh)

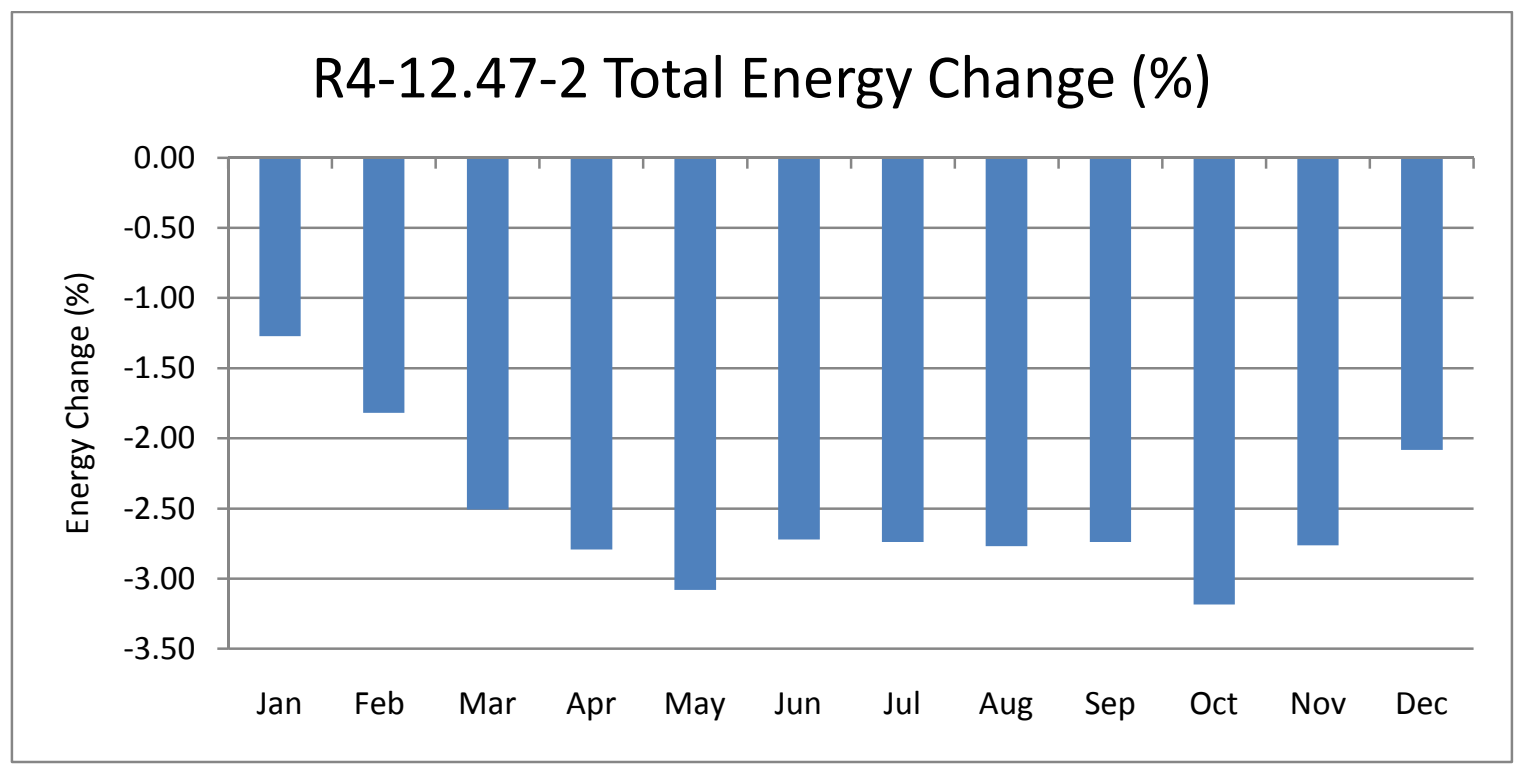

Figure 6.92: R4-12.47-2 Total Energy Change (\%) 


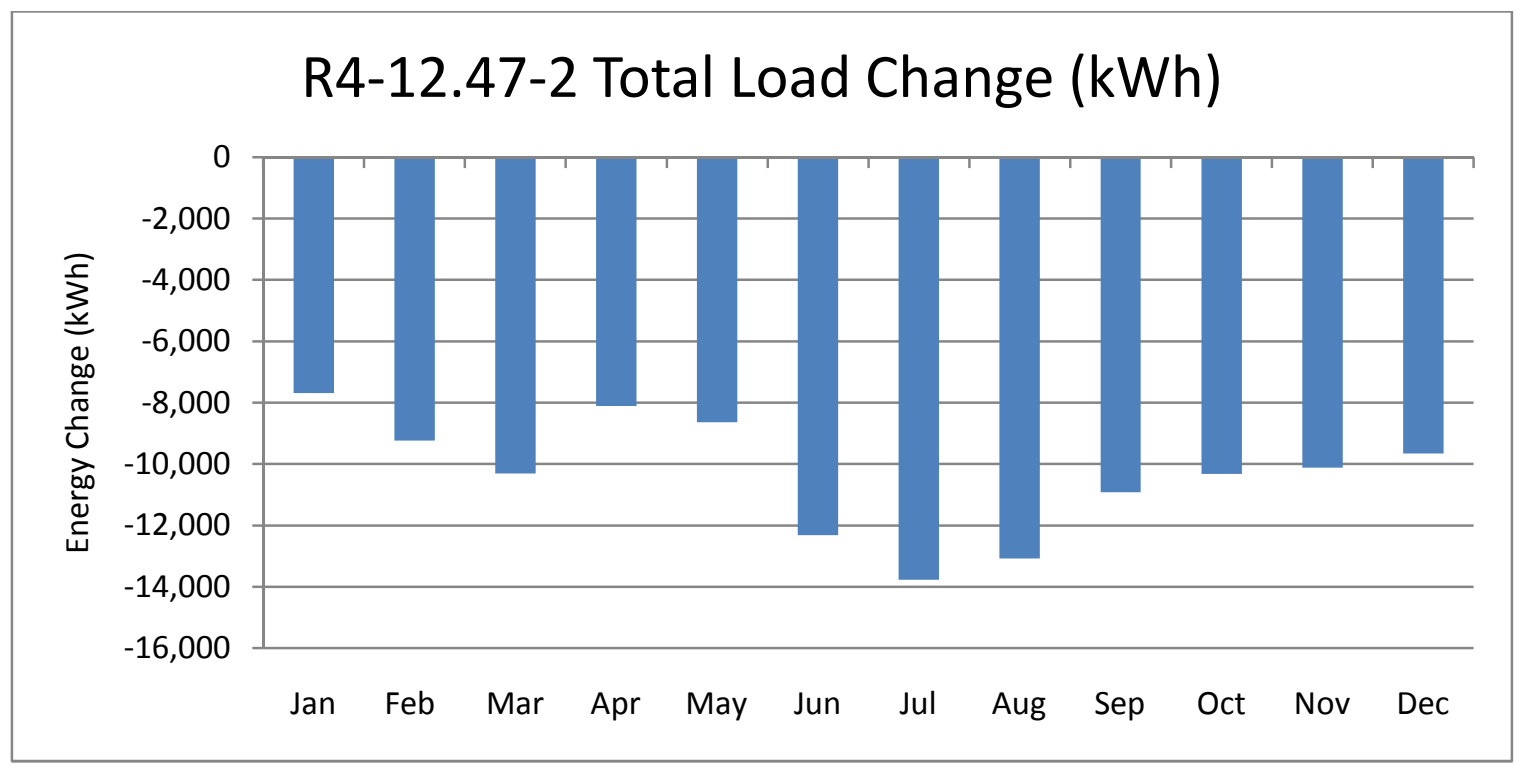

Figure 6.93: R4-12.47-2 Total Load Change (kWh)

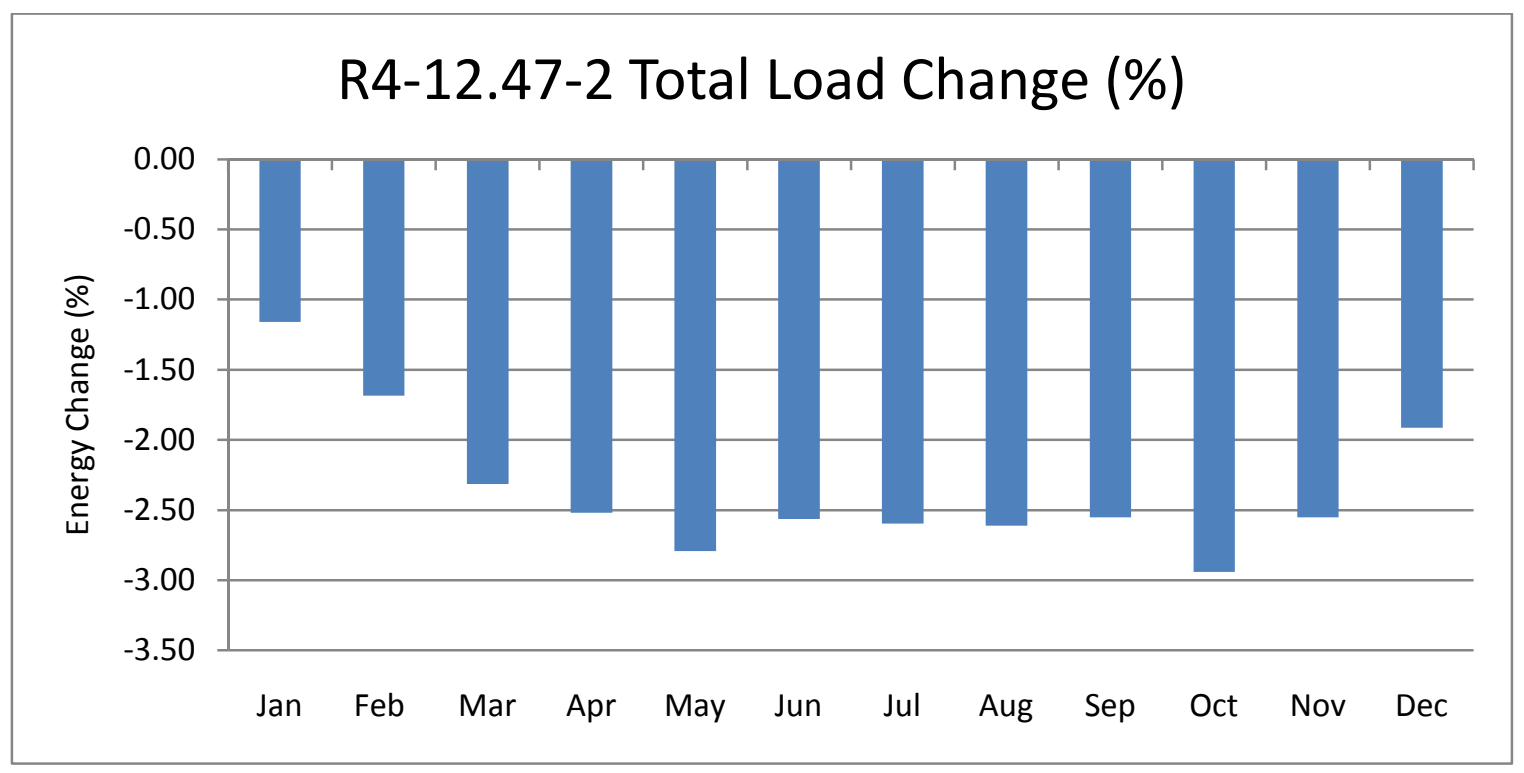

Figure 6.94: R4-12.47-2 Total Load Change (kWh) 


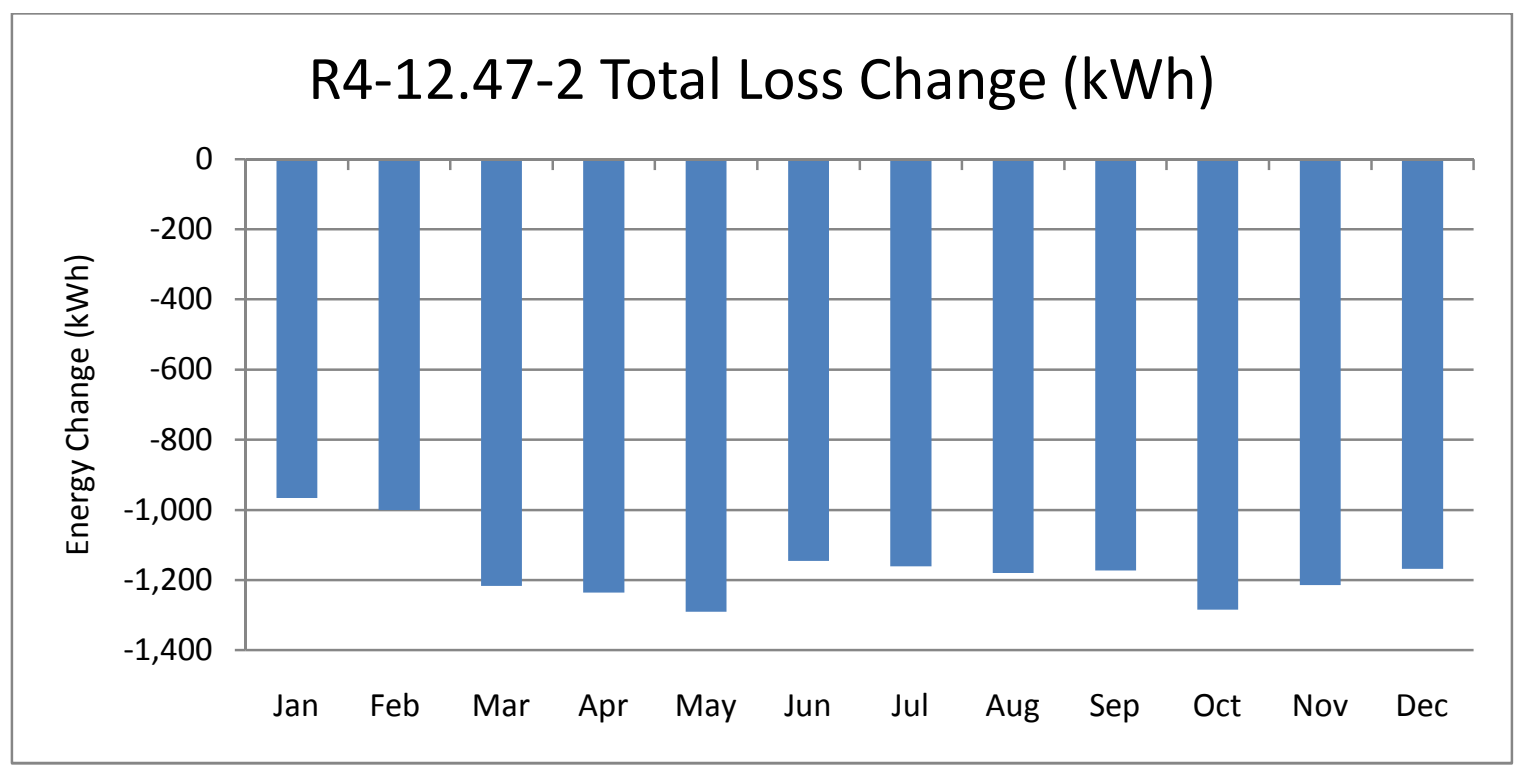

Figure 6.95: R4-12.47-2 Total Loss Change (kWh)

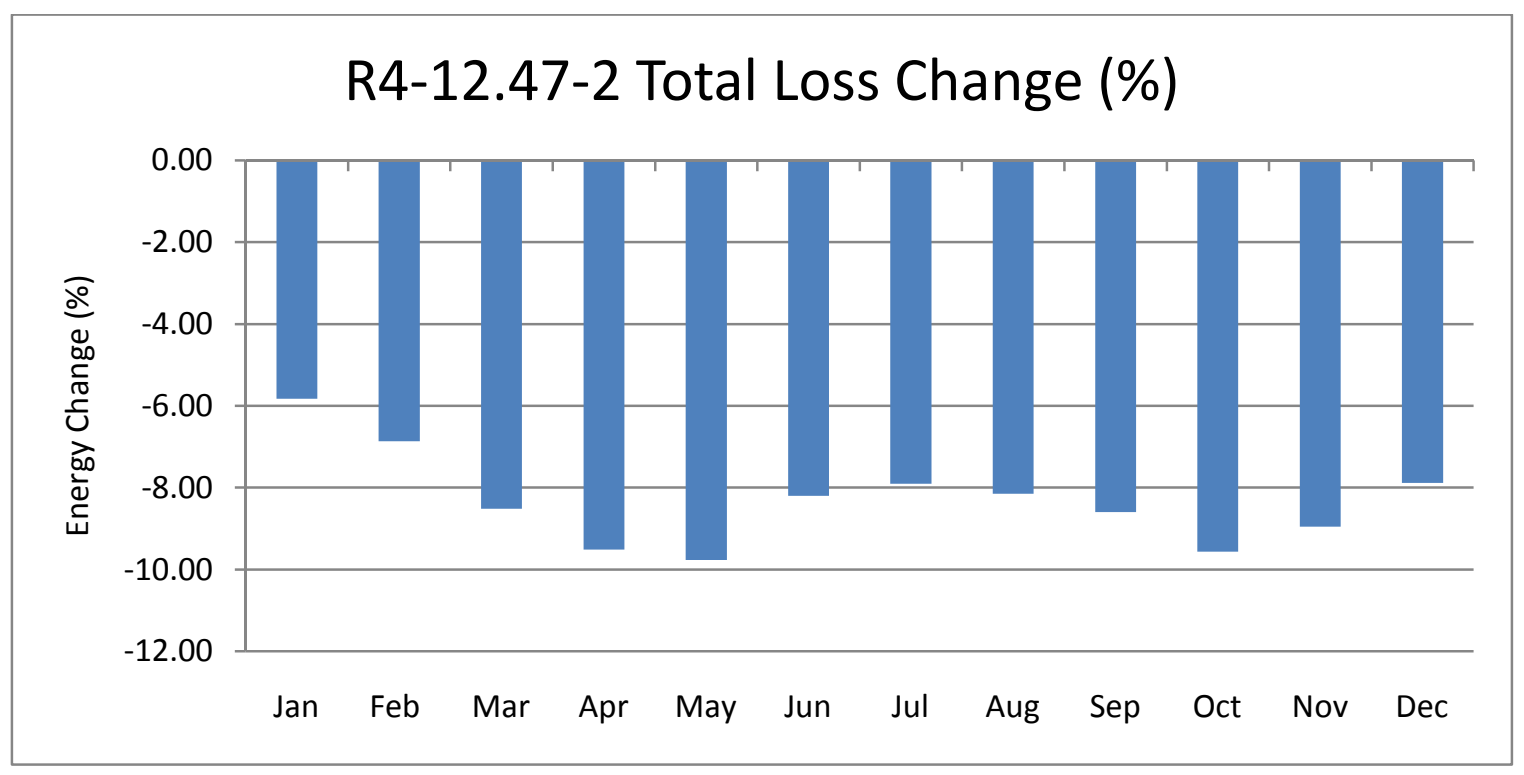

Figure 6.96: R4-12.47-2 Total Loss Change (\%) 


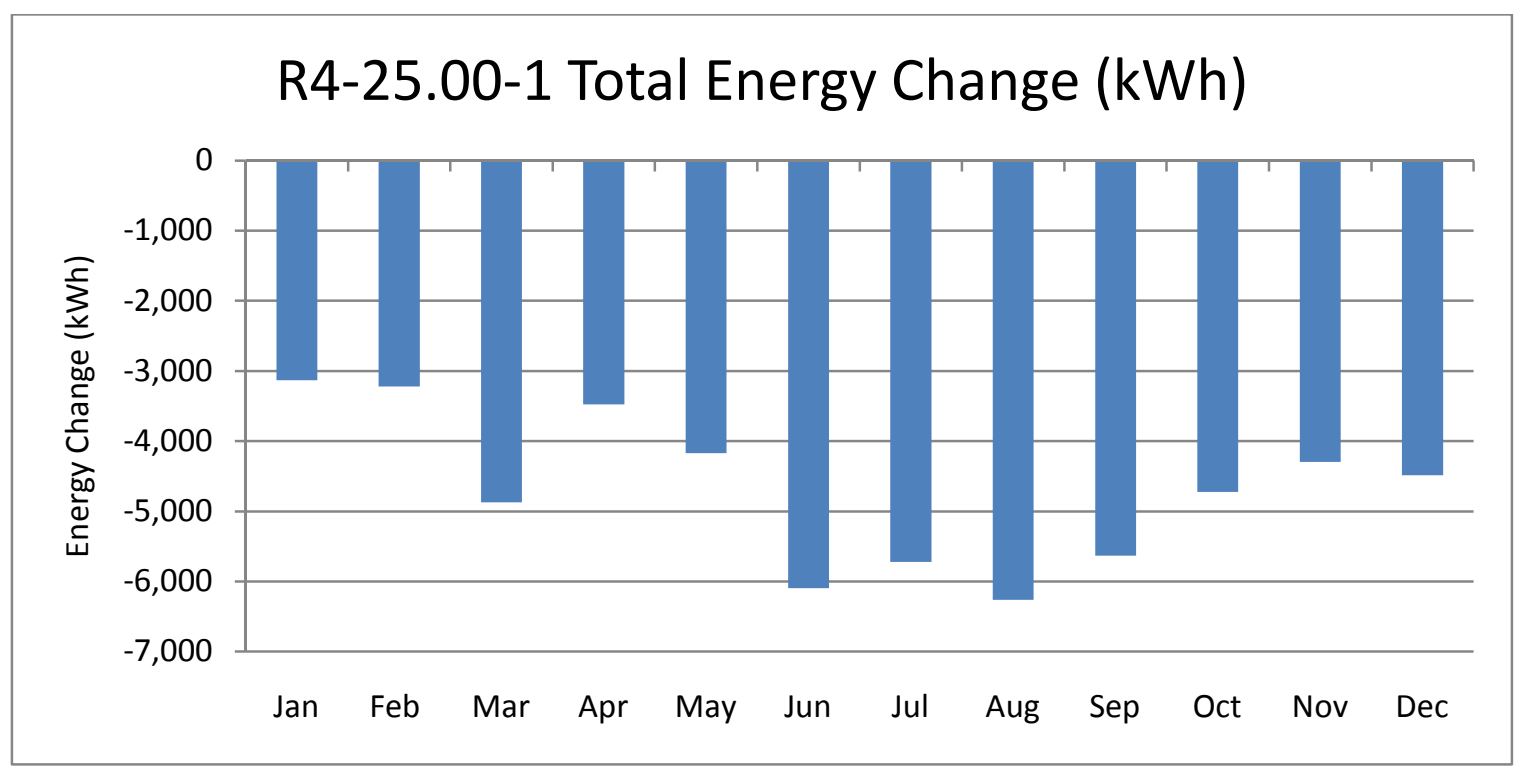

Figure 6.97: R4-25.00-1 Total Energy Change (kWh)

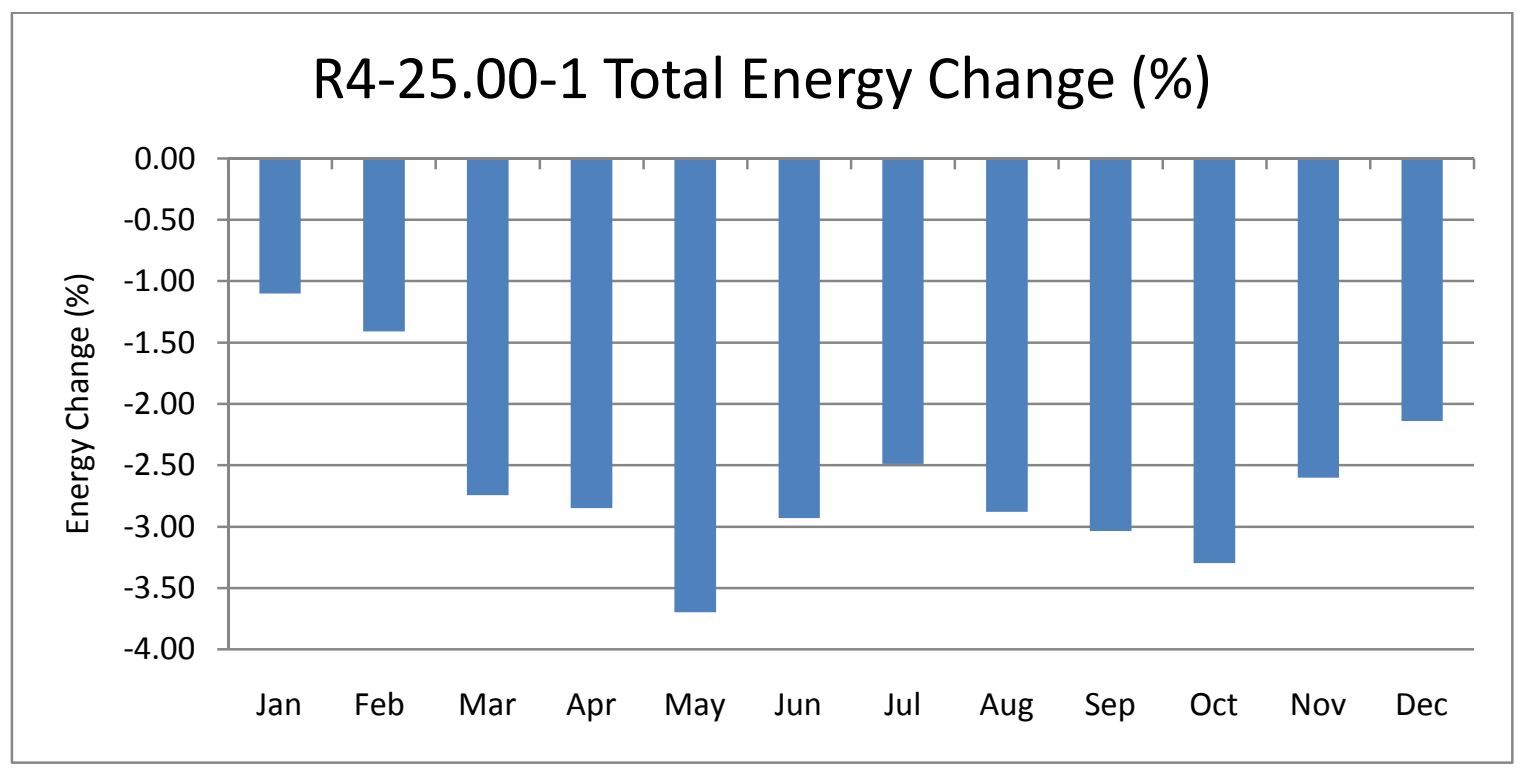

Figure 6.98: R4-25.00-1 Total Energy Change (\%) 


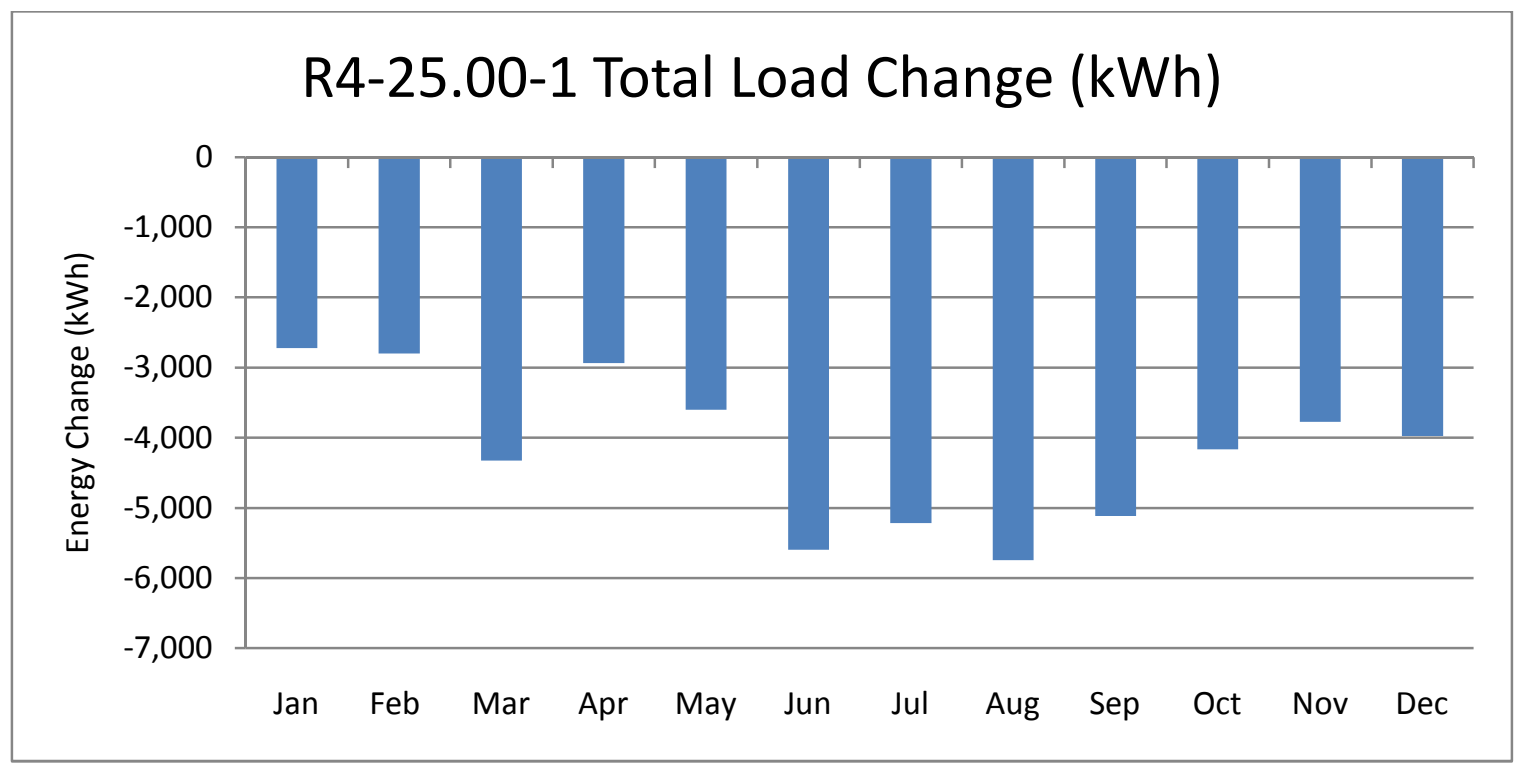

Figure 6.99: R4-25.00-1 Total Load Change (kWh)

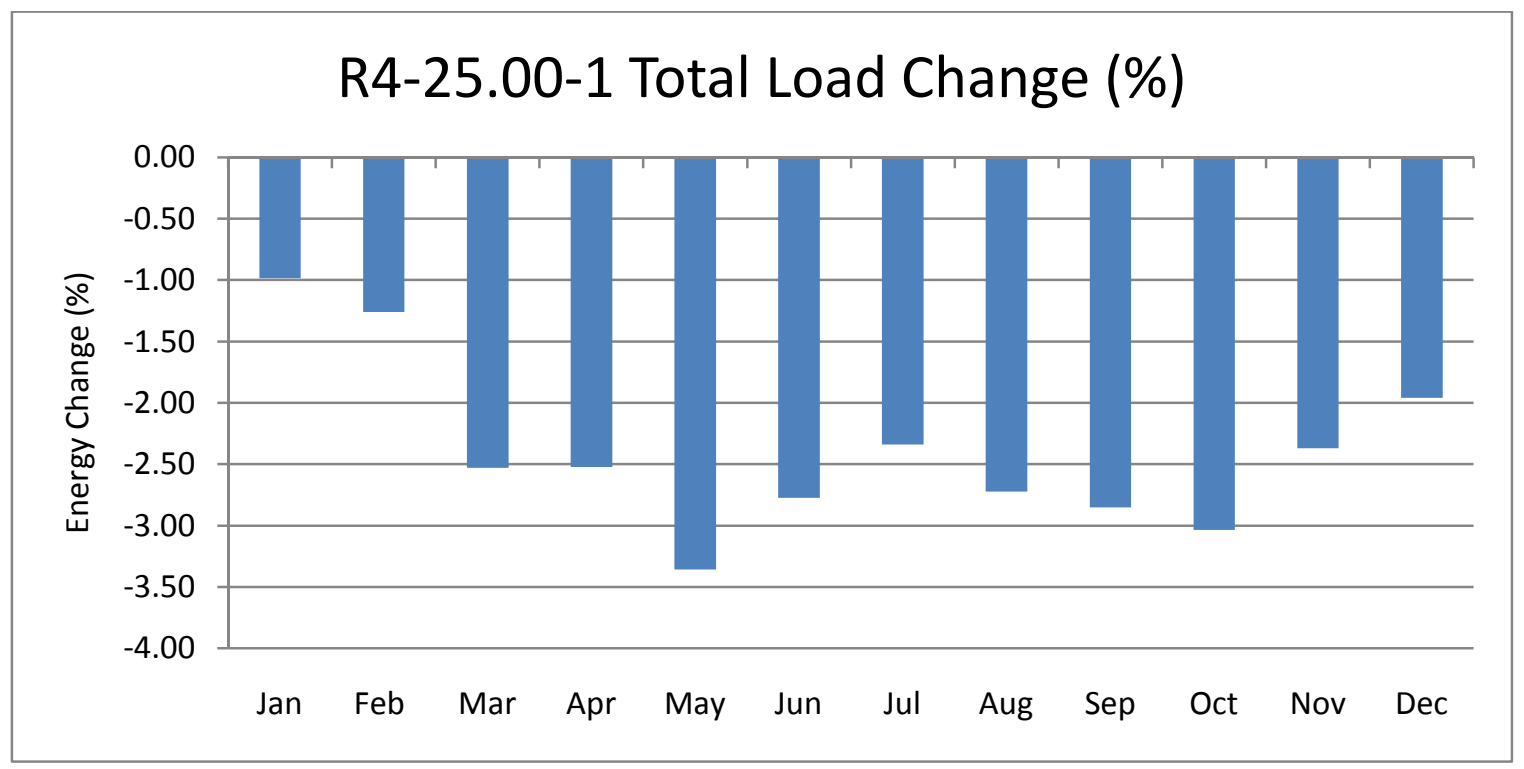

Figure 6.100: R4-25.00-1 Total Load Change (\%) 


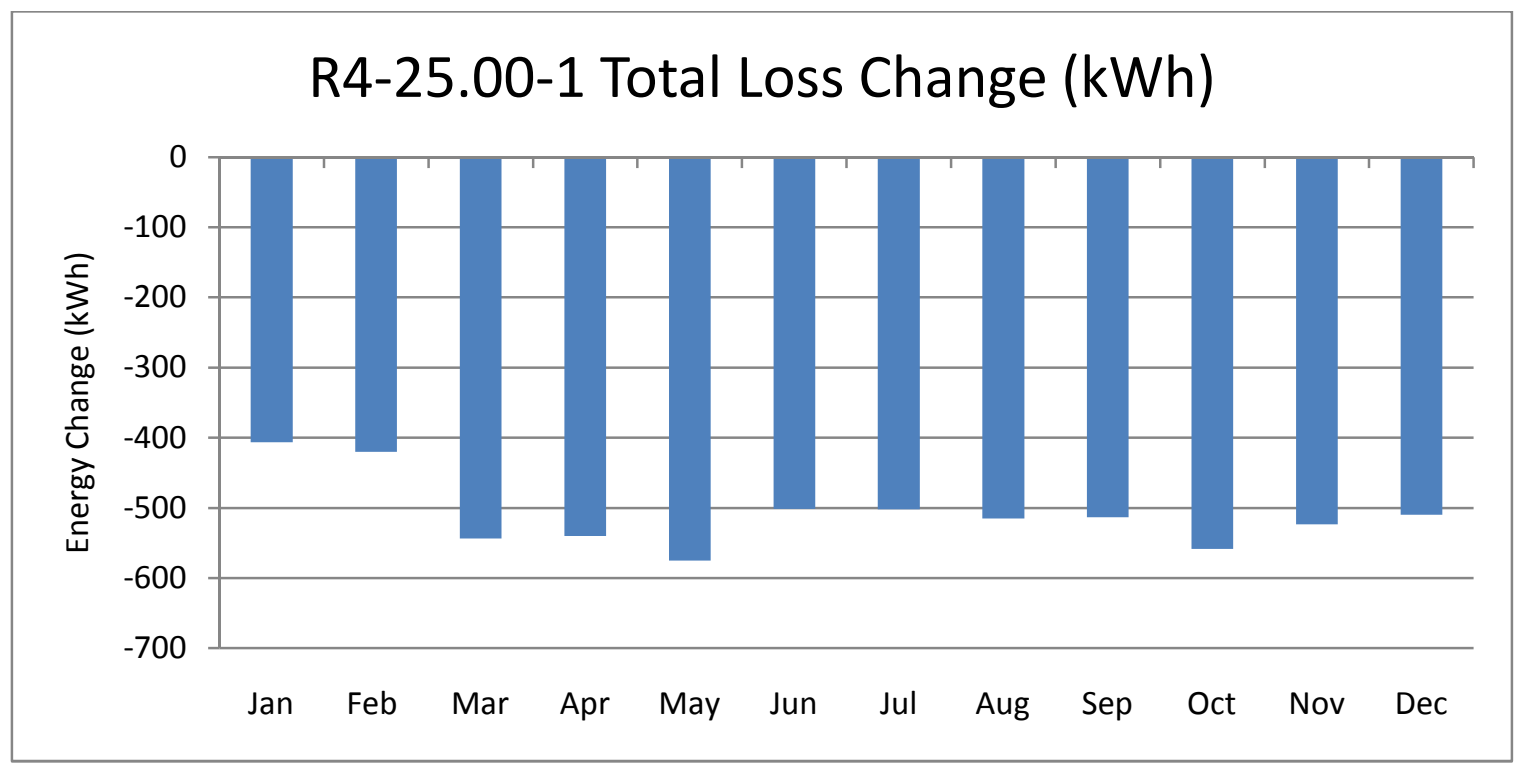

Figure 6.101: R4-25.00-1 Total Loss Change (kWh)

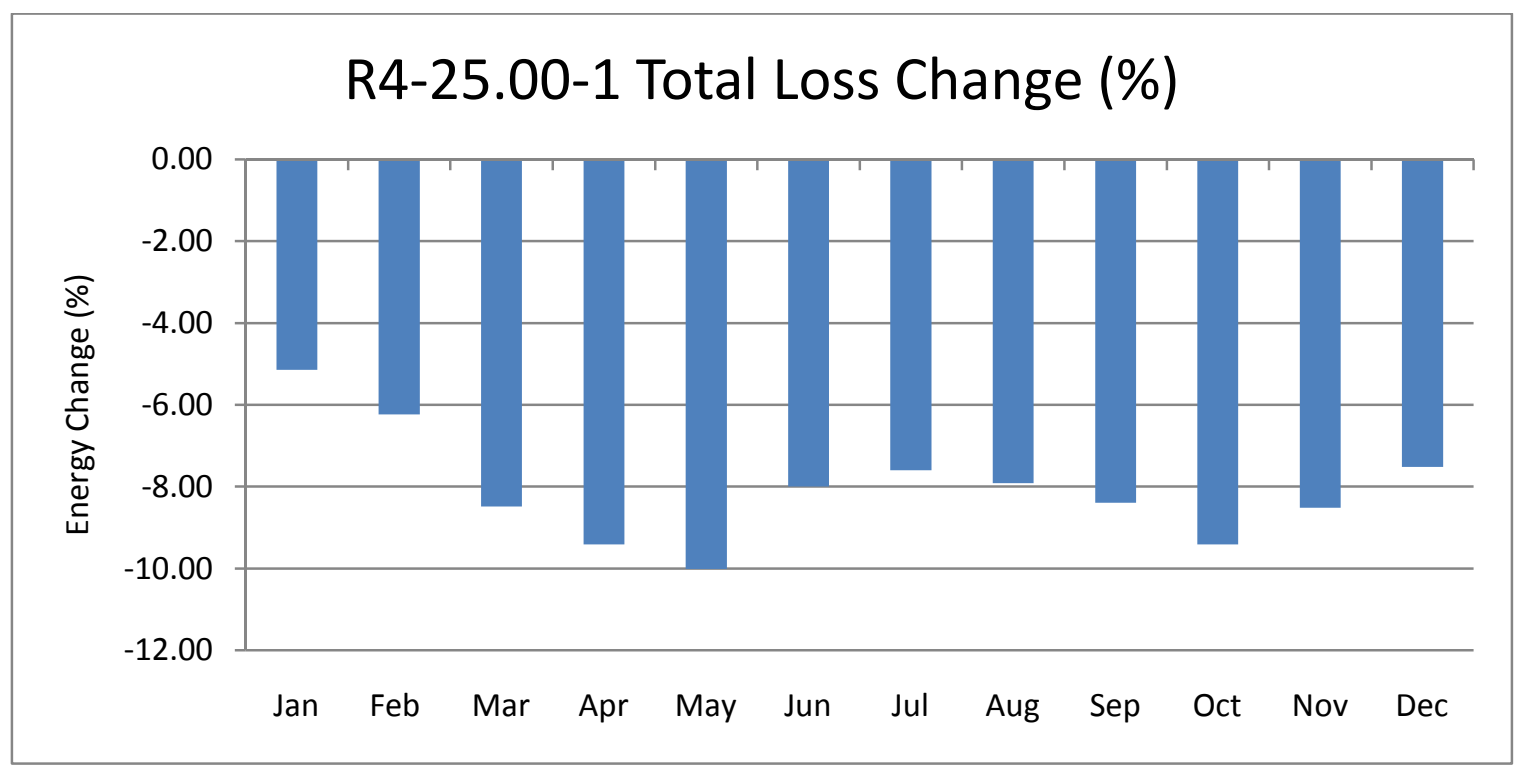

Figure 6.102: R4-25.00-1 Total Loss Change (\%)

6.5. Region 5: CVR Plots 


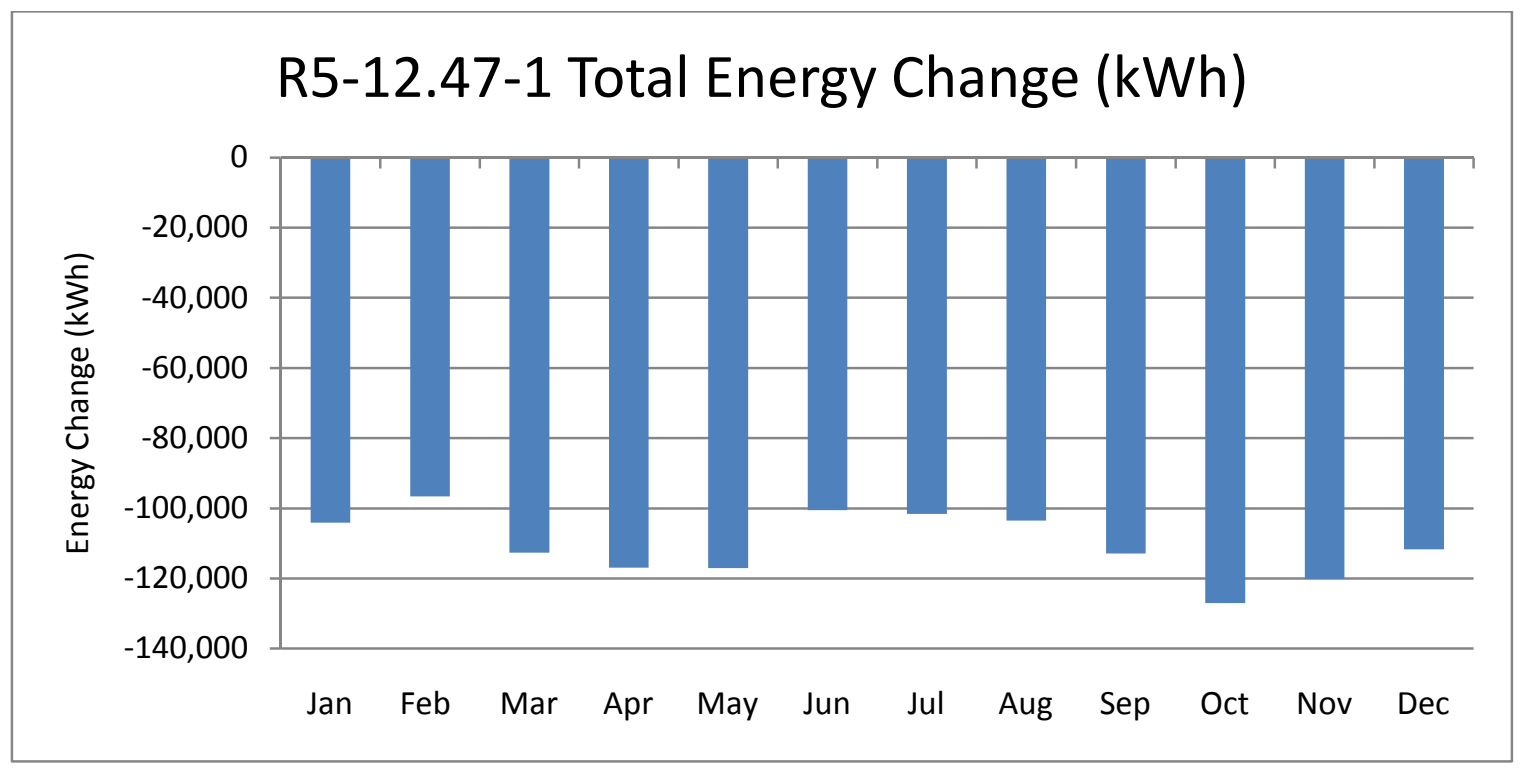

Figure 6.103: R5-12.47-1 Total Energy Change (kWh)

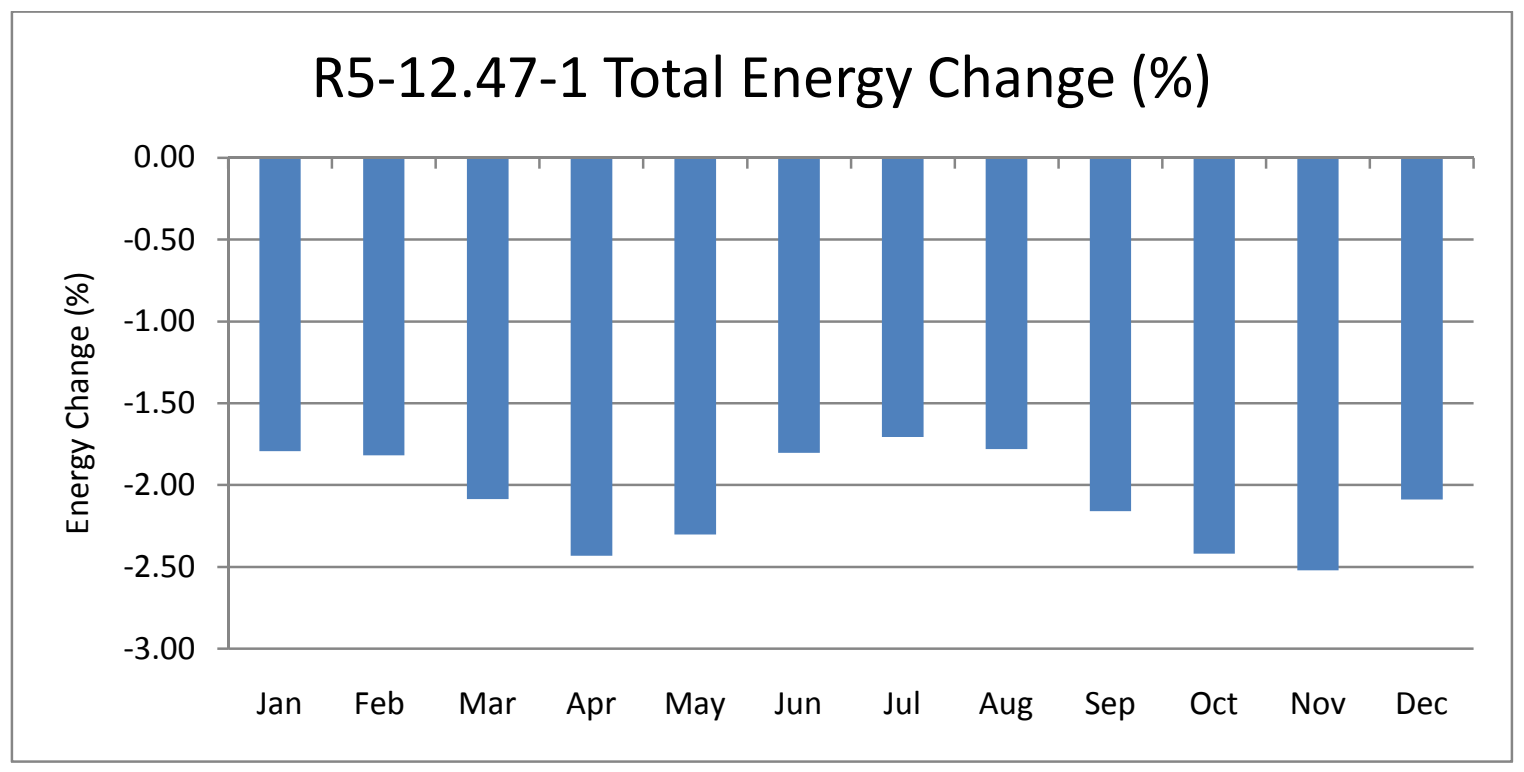

Figure 6.104: R5-12.47-1 Total Energy Change (\%) 


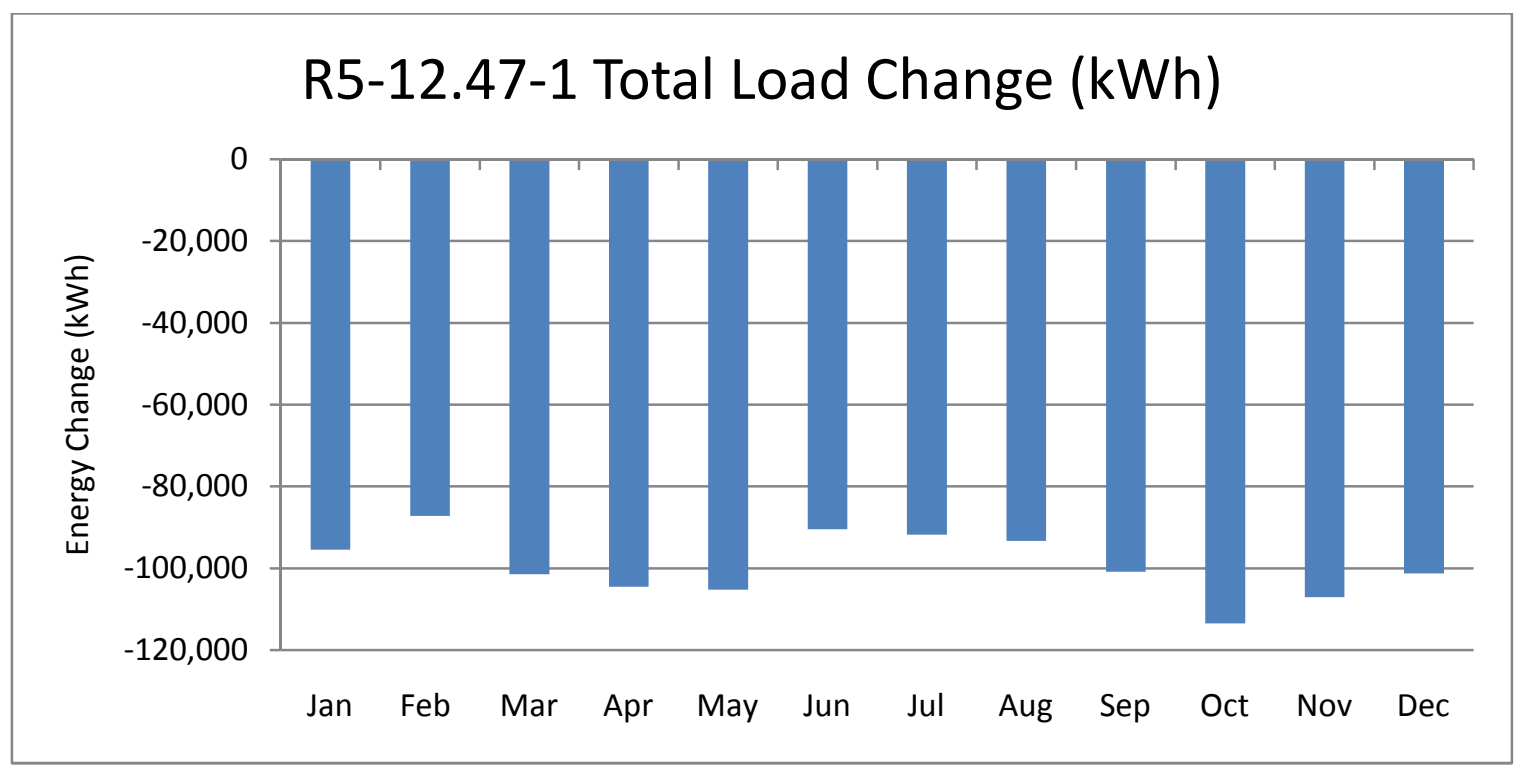

Figure 6.105: R5-12.47-1 Total Load Change (kWh)

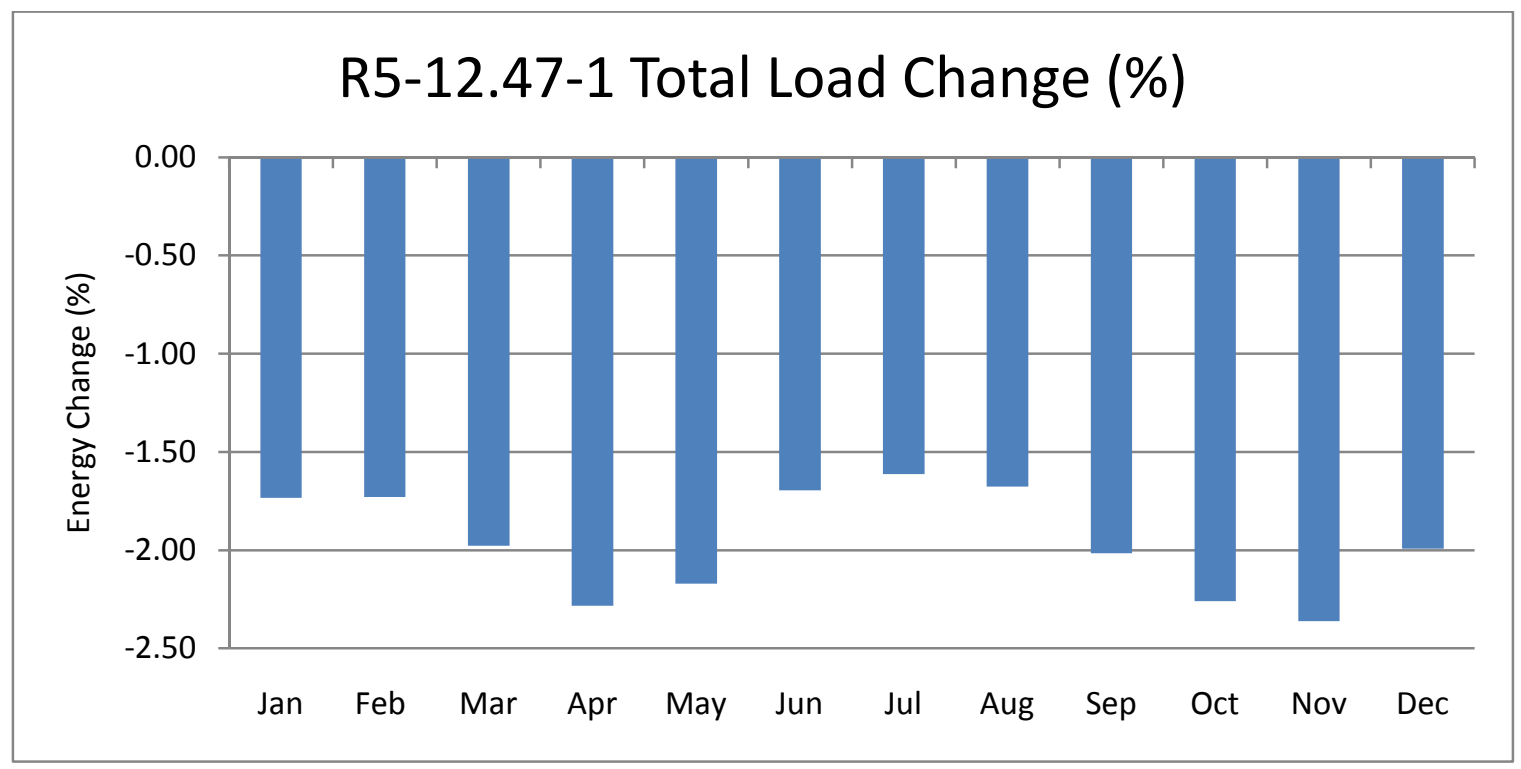

Figure 6.106: R5-12.47-1 Total Load Change (\%) 


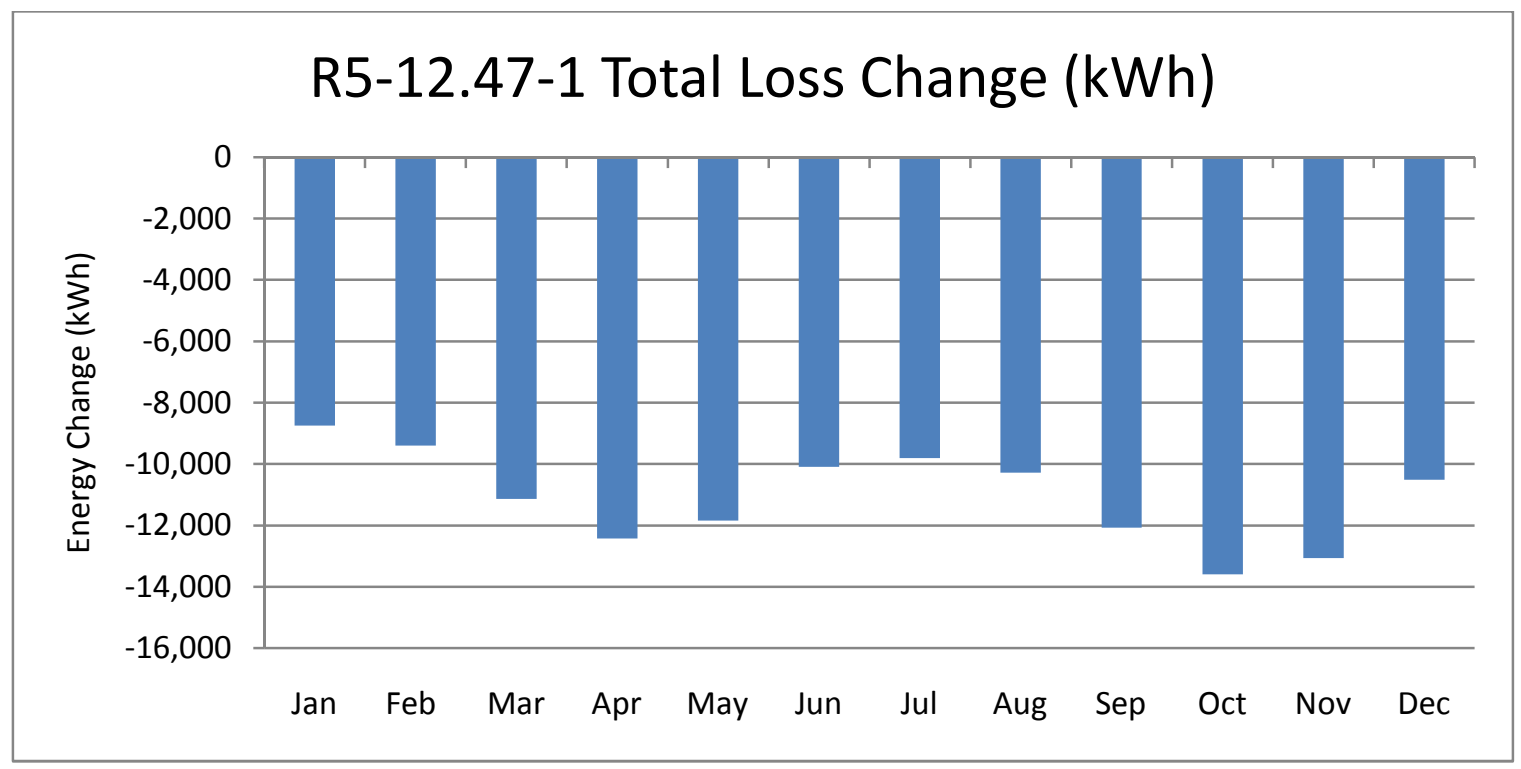

Figure 6.107: R5-12.47-1 Total loss Change (kWh)

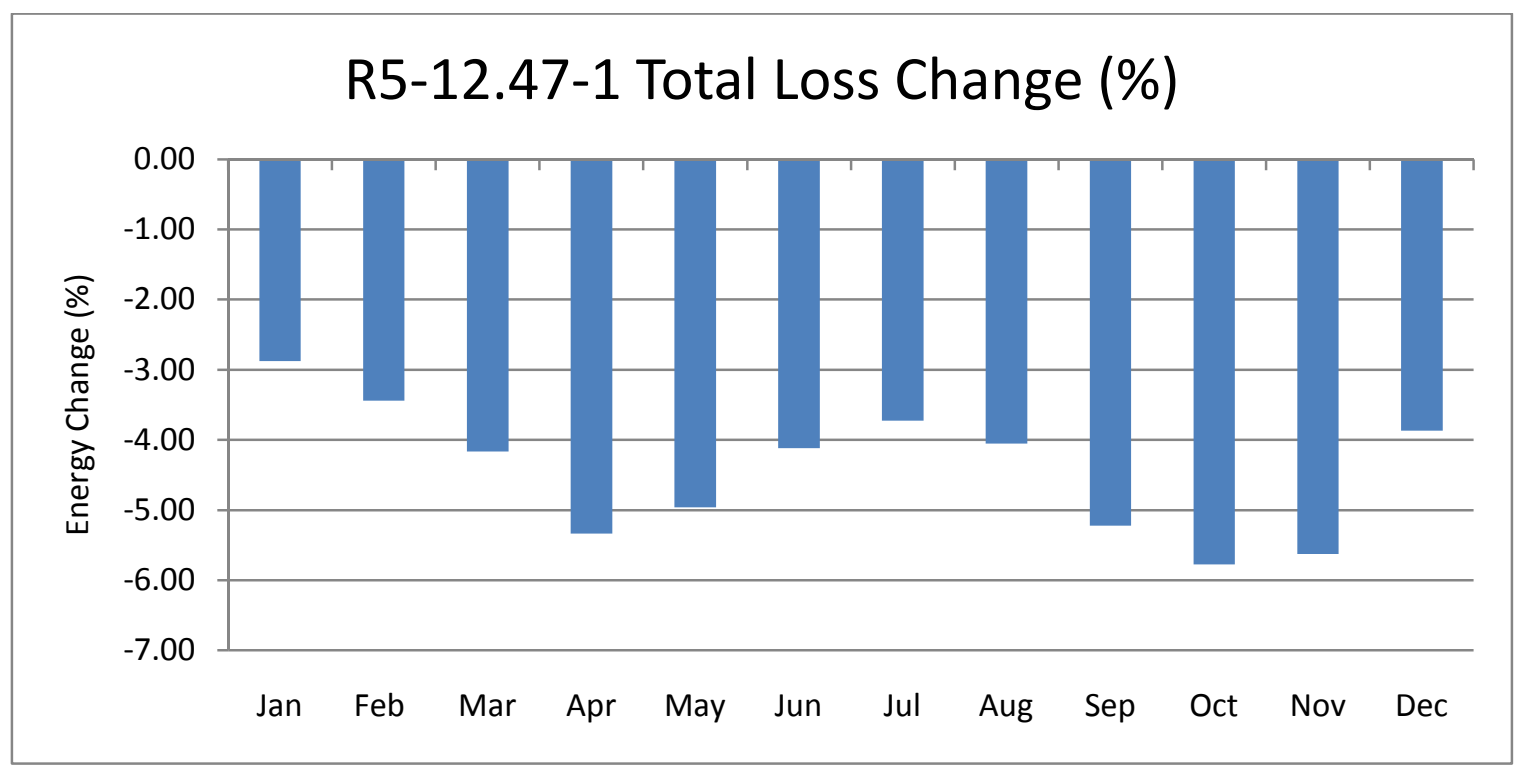

Figure 6.108: R5-12.47-1 Total Loss Change (\%) 


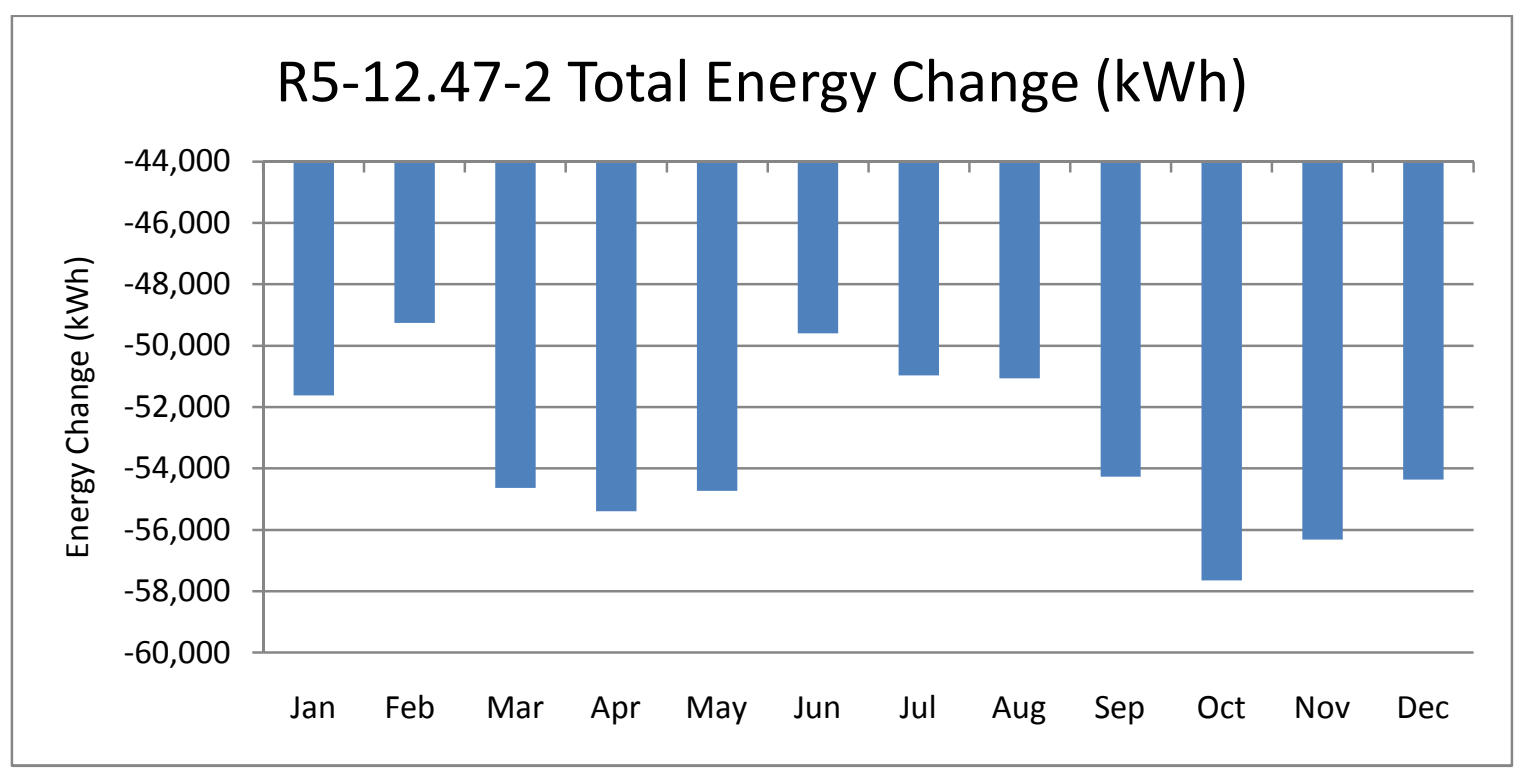

Figure 6.109: R5-12.47-2 Total Energy Change (kWh)

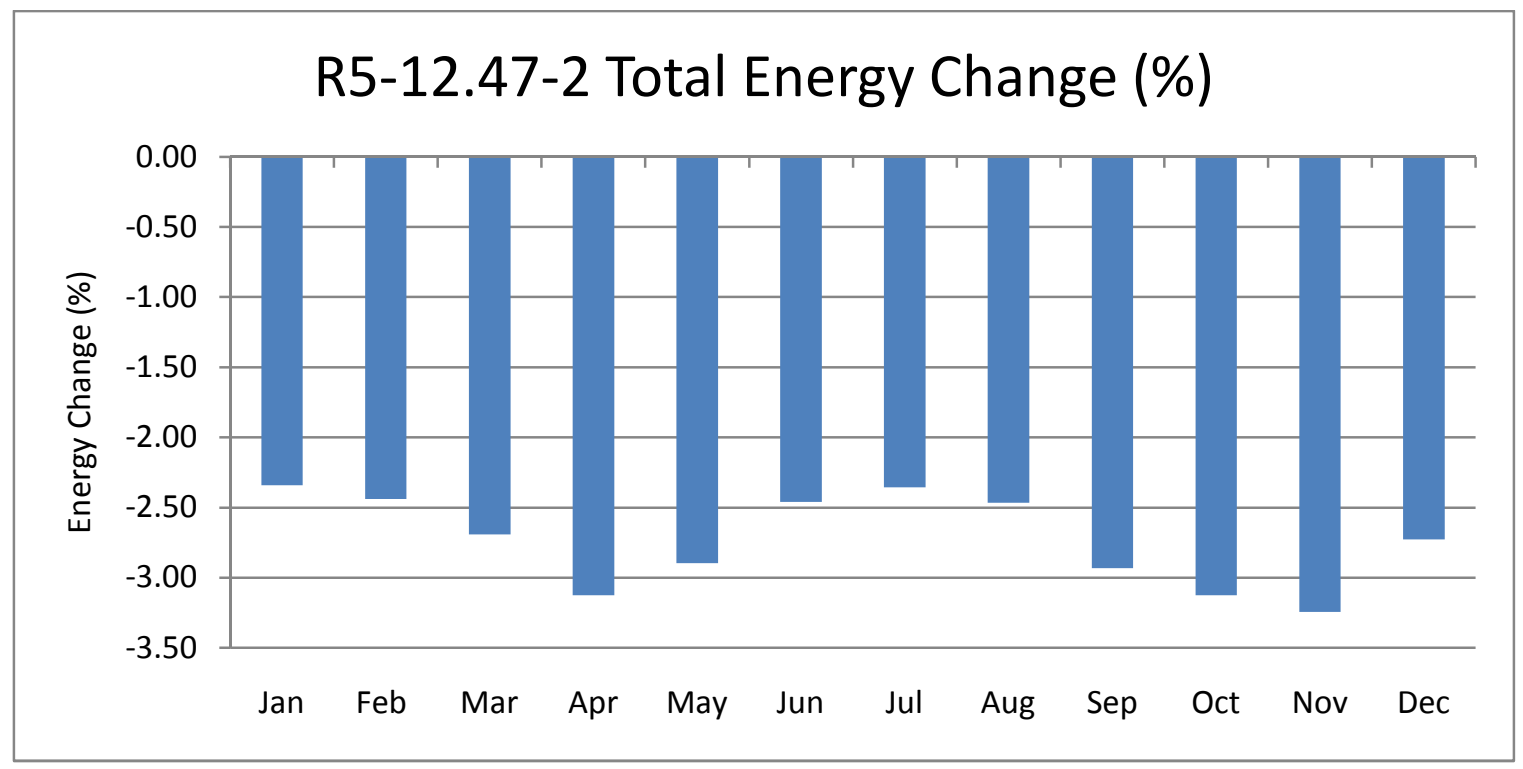

Figure 6.110: R5-12.47-2 Total Energy Change (\%) 


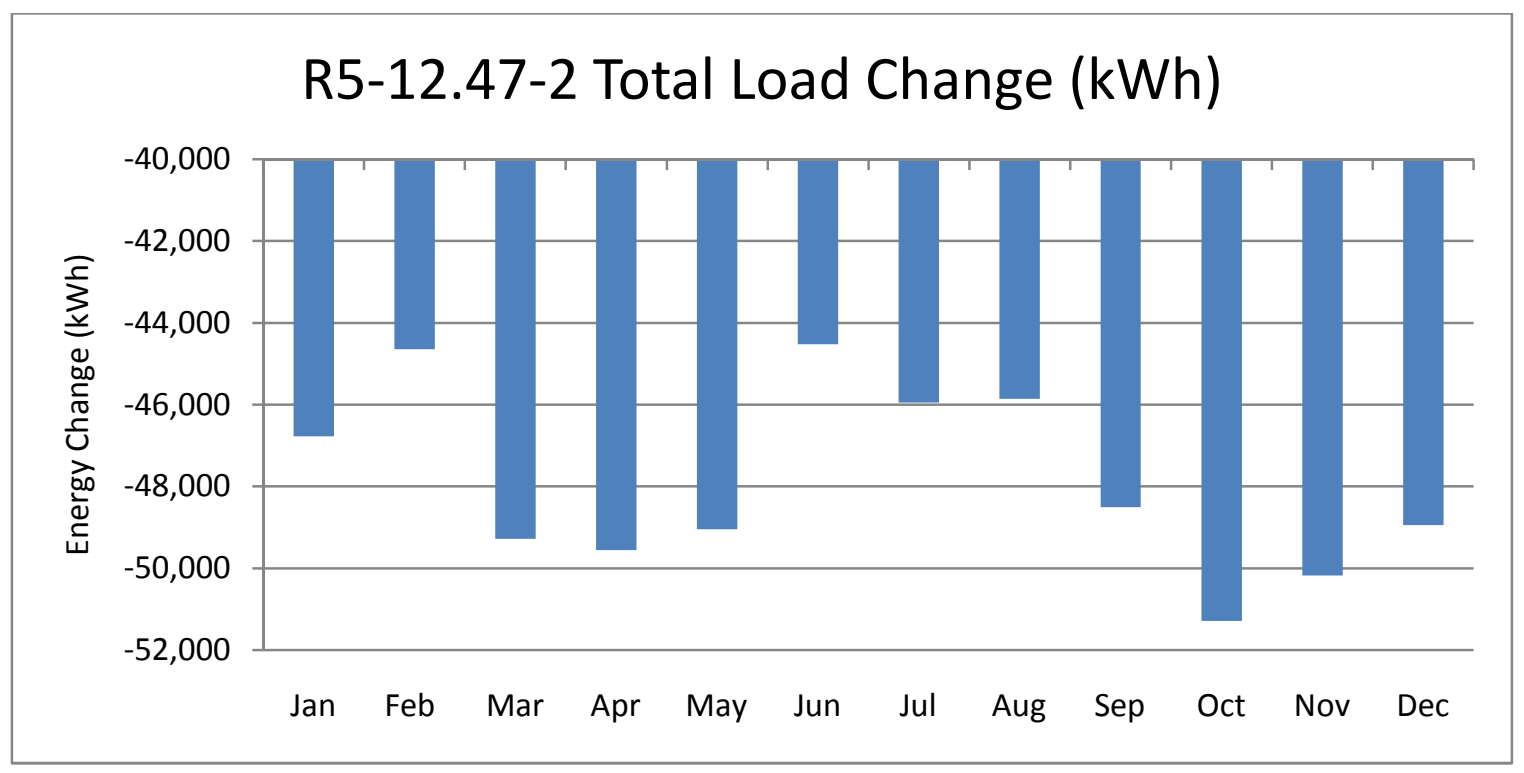

Figure 6.111: R5-12.47-2 Total Load Change (kWh)

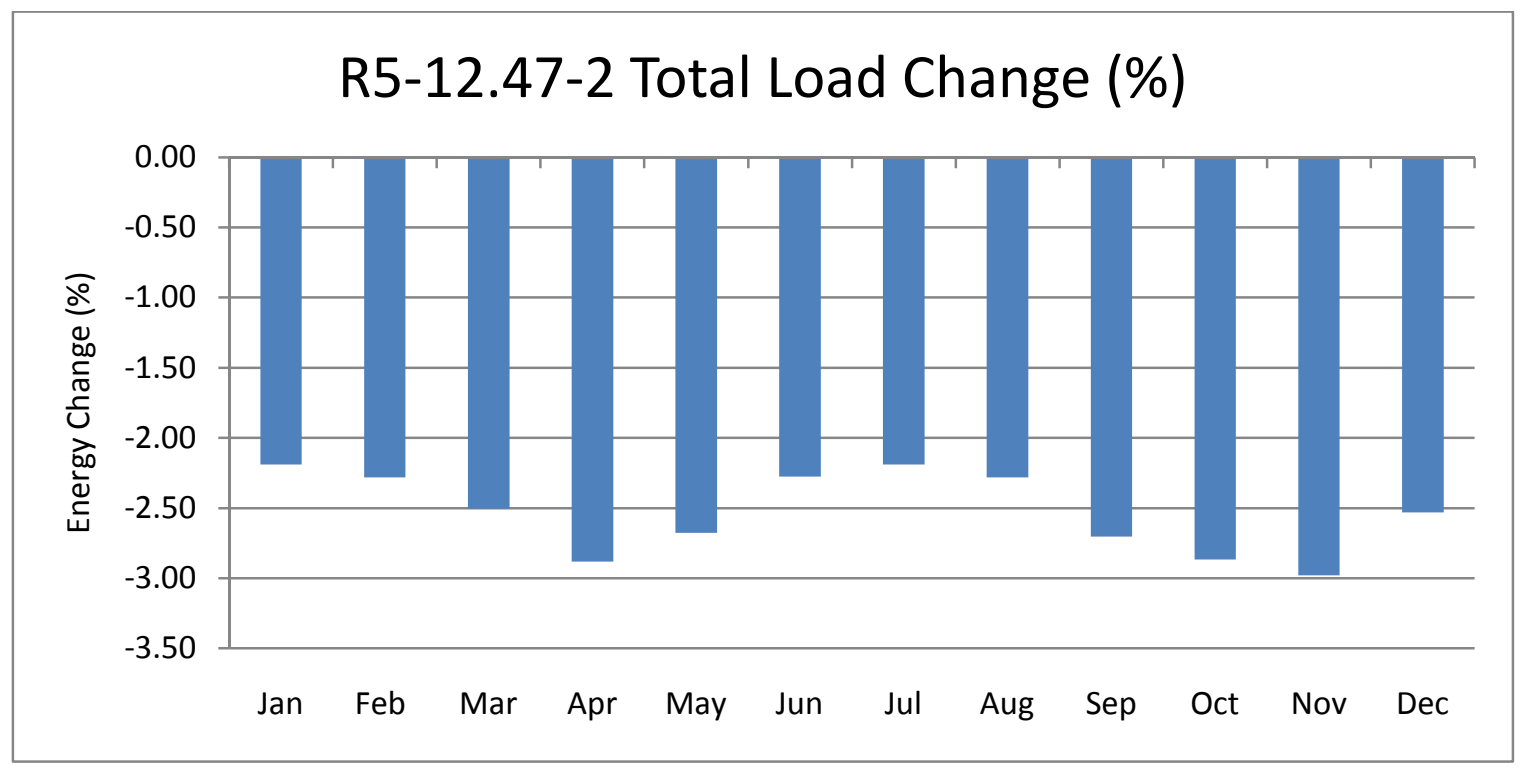

Figure 6.112: R5-12.47-2 Total Load Change (\%) 


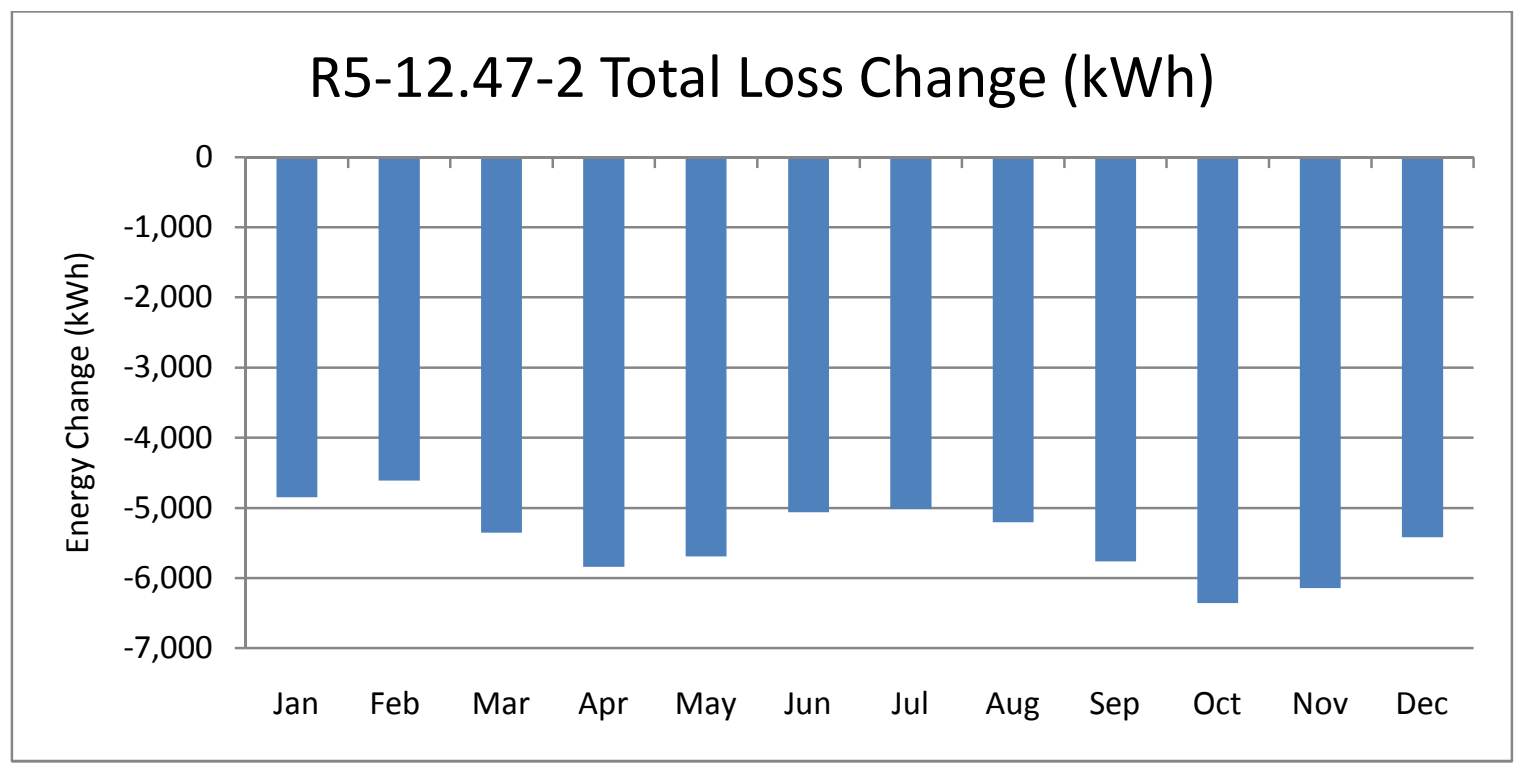

Figure 6.113: R5-12.47-2 Total Loss Change (kWh)

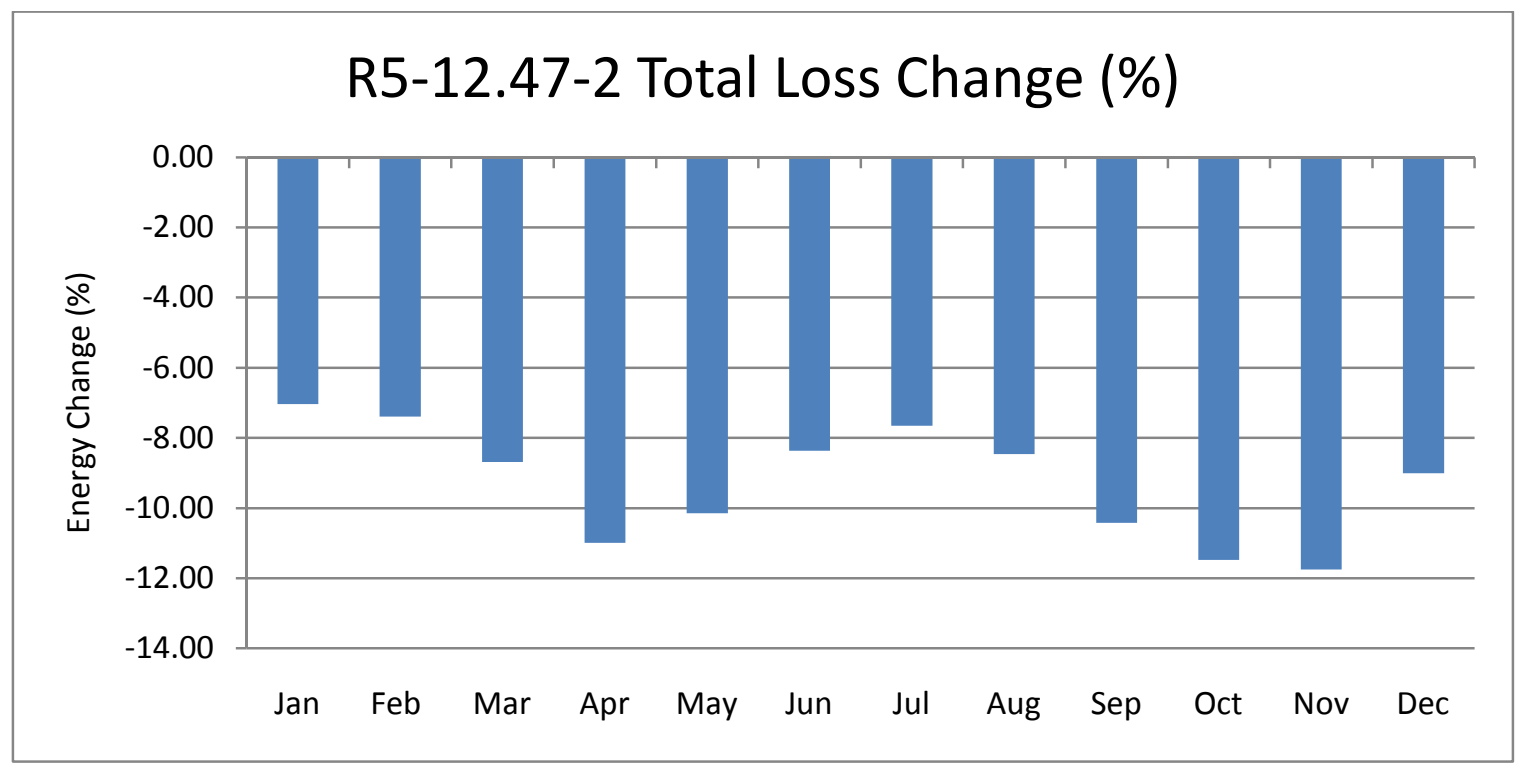

Figure 6.114: R5-12.47-2 Total Loss Change (\%) 


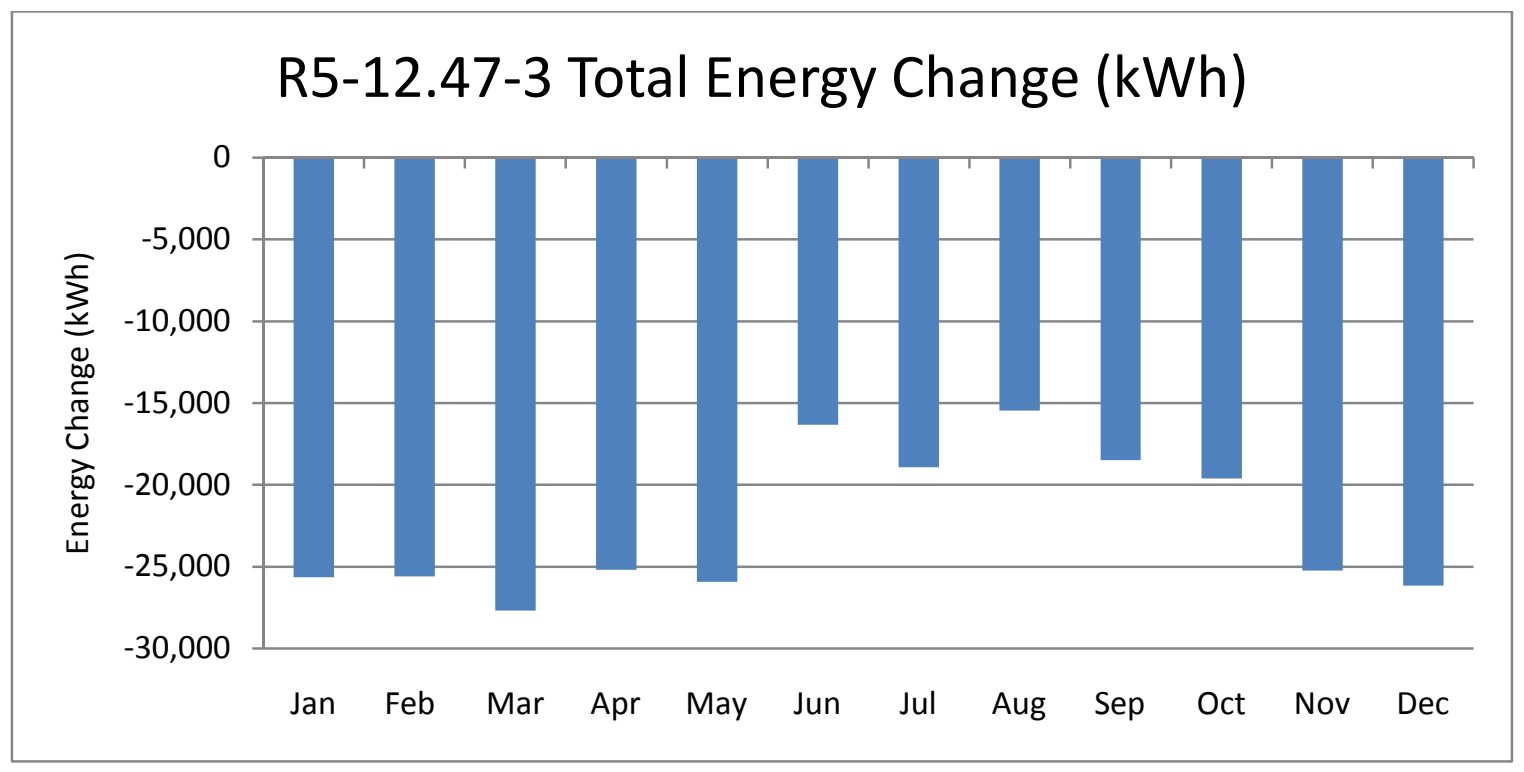

Figure 6.115: R5-12.47-3 Total Energy Change (kWh)

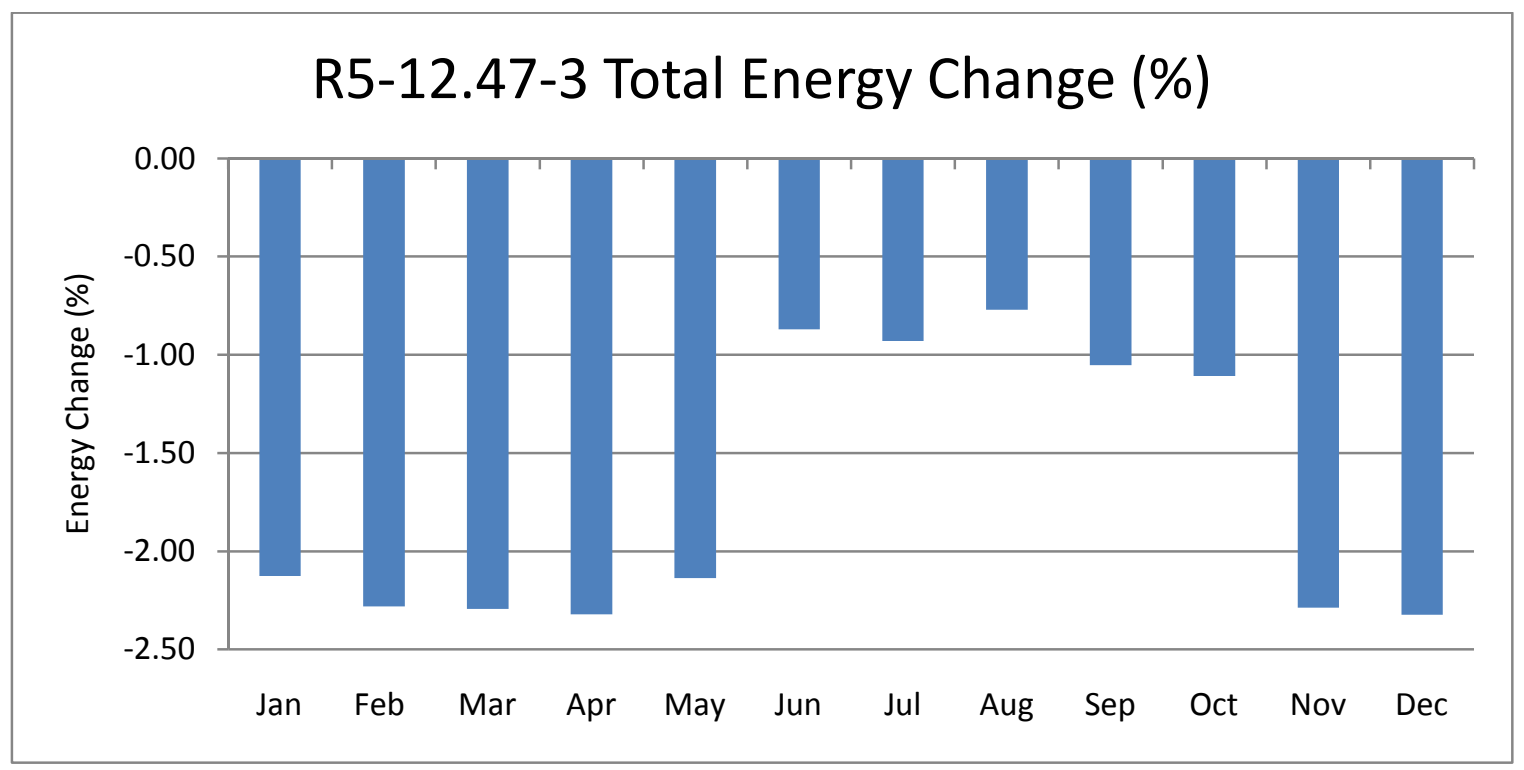

Figure 6.116: R5-12.47-3 Total Energy Change (\%) 


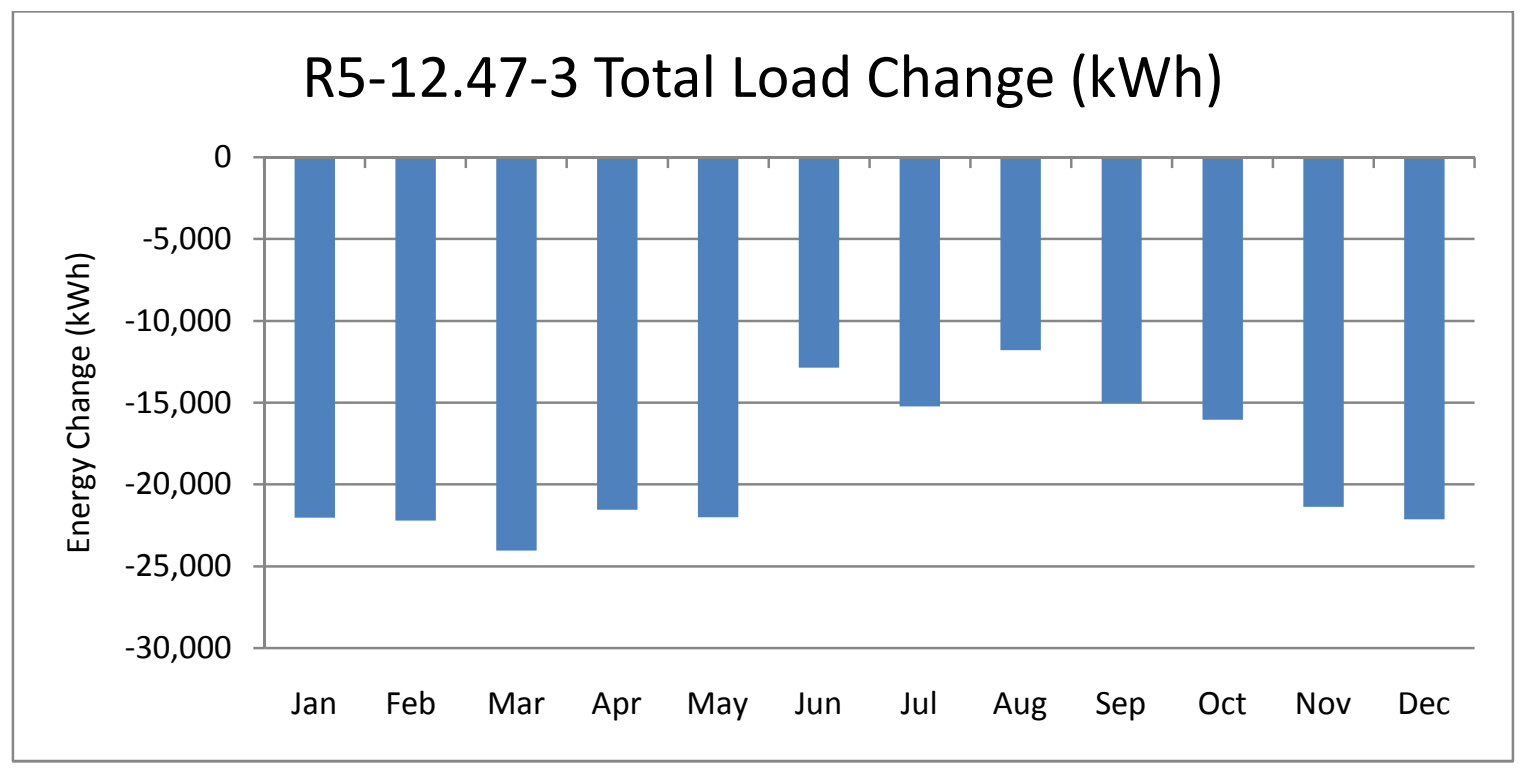

Figure 6.117: R5-12.47-3 Total Load Change (kWh)

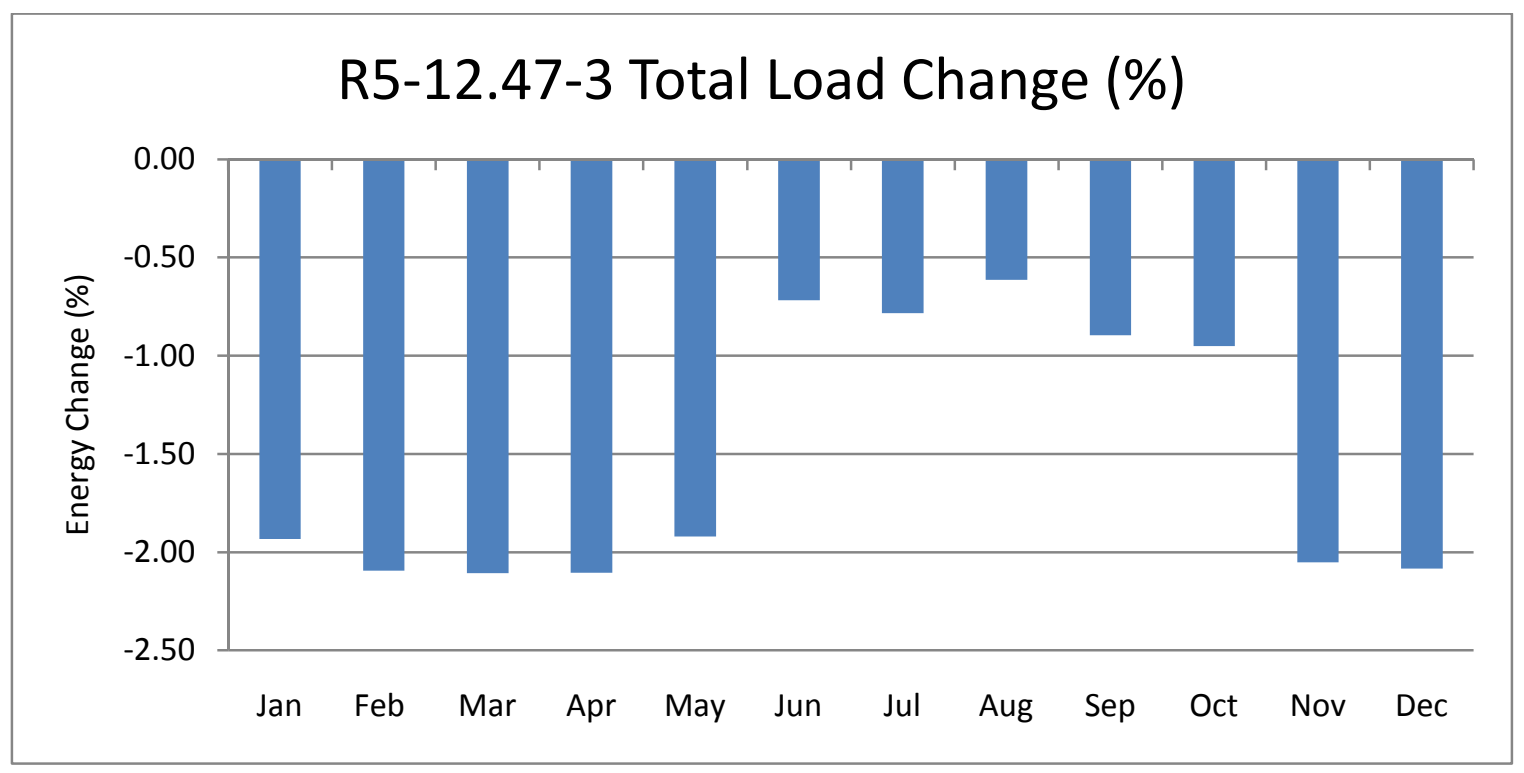

Figure 6.118: R5-12.47-3 Total Load Change (\%) 


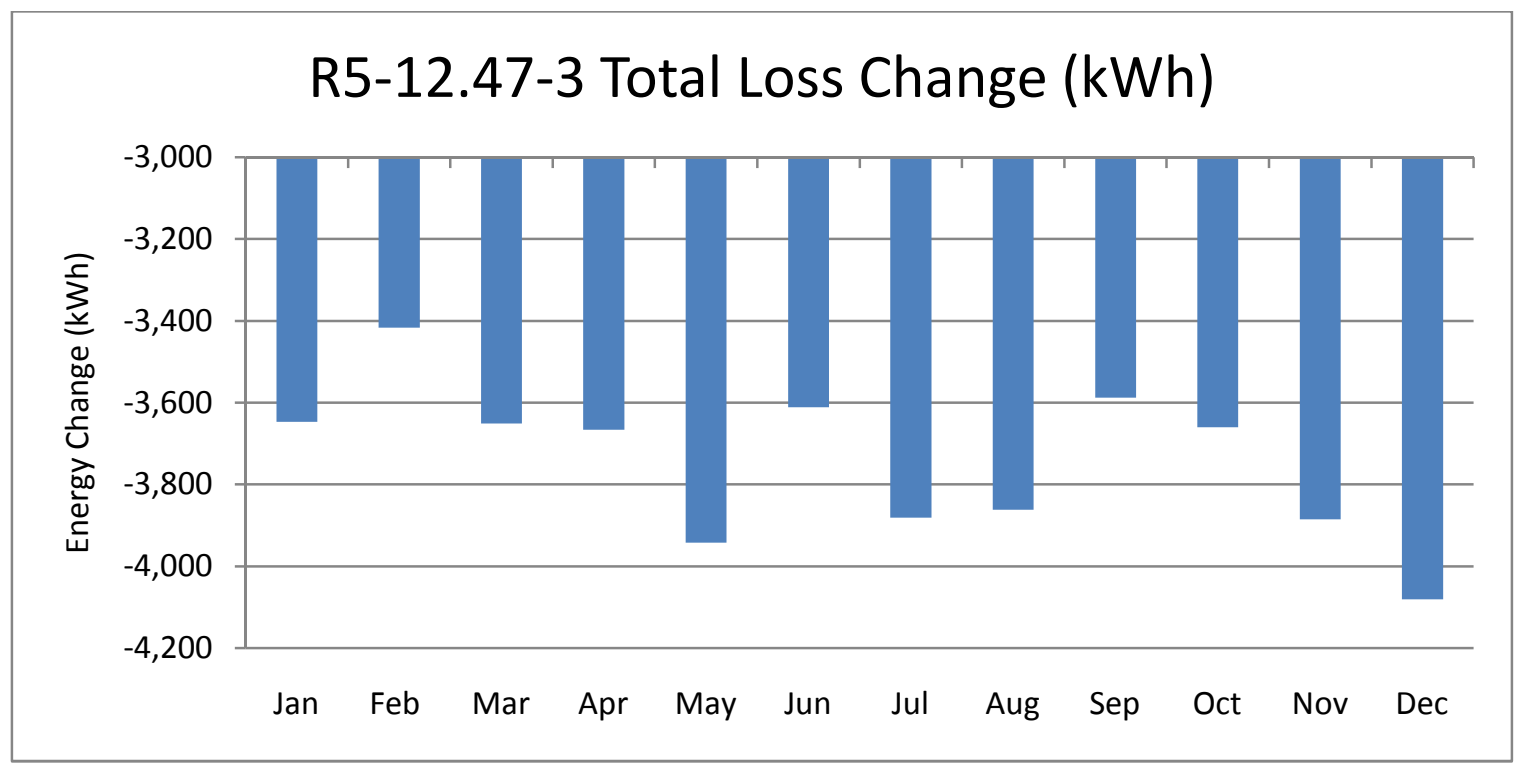

Figure 6.119: R5-12.47-3 Total Loss Change (kWh)

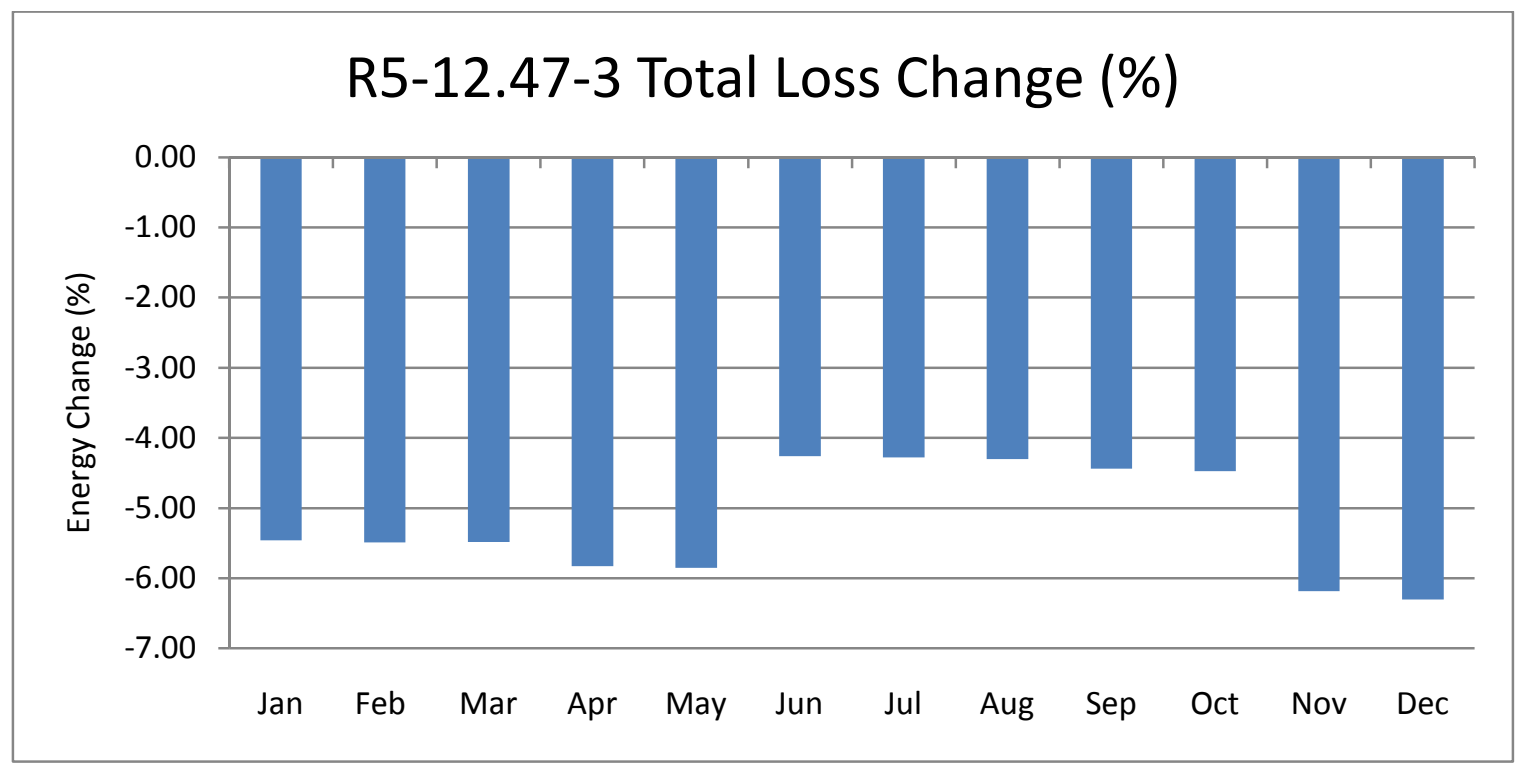

Figure 6.120: R5-12.47-3 Total Loss Change (\%) 


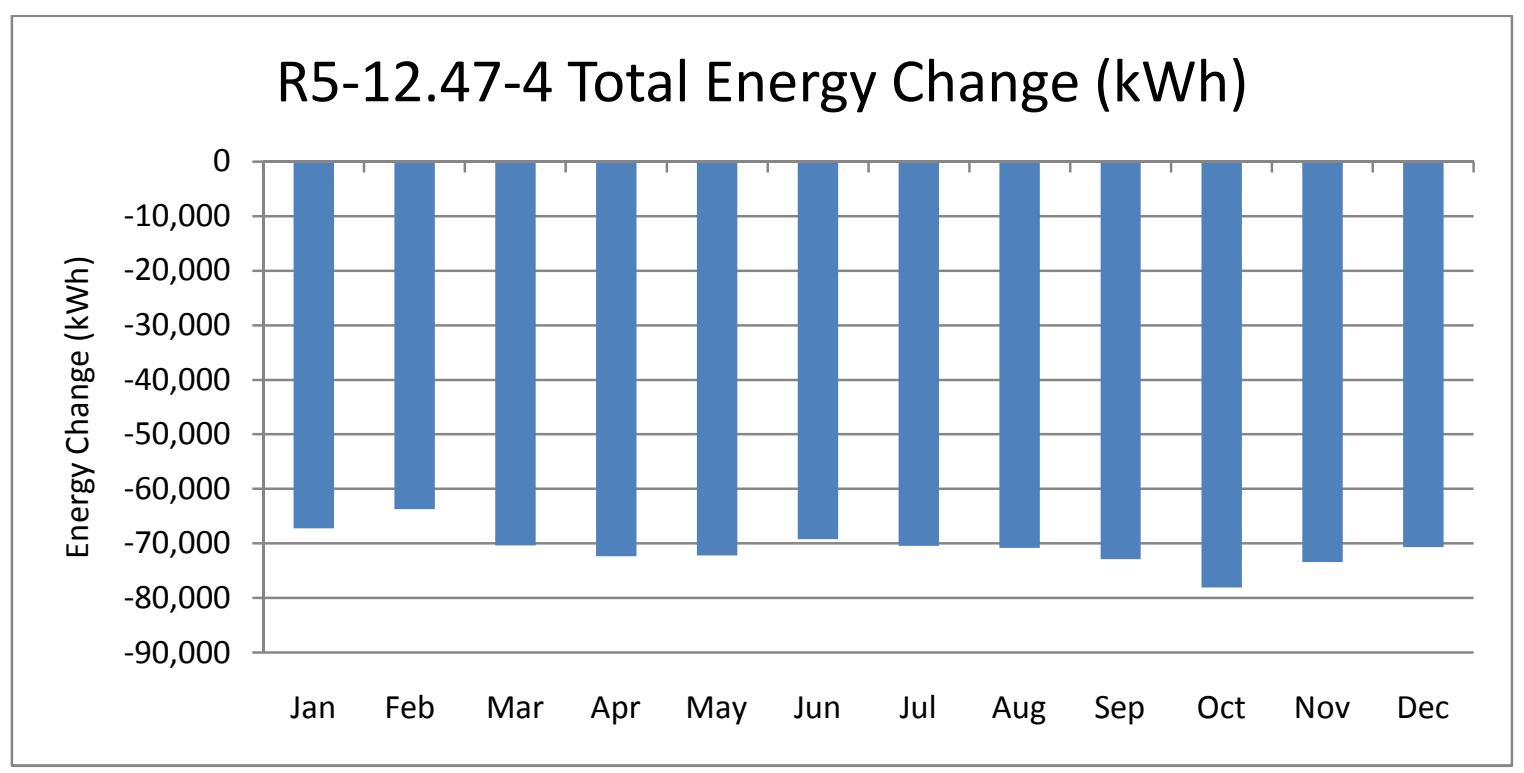

Figure 6.121: R5-12.47-4 Total Energy Change (kWh)

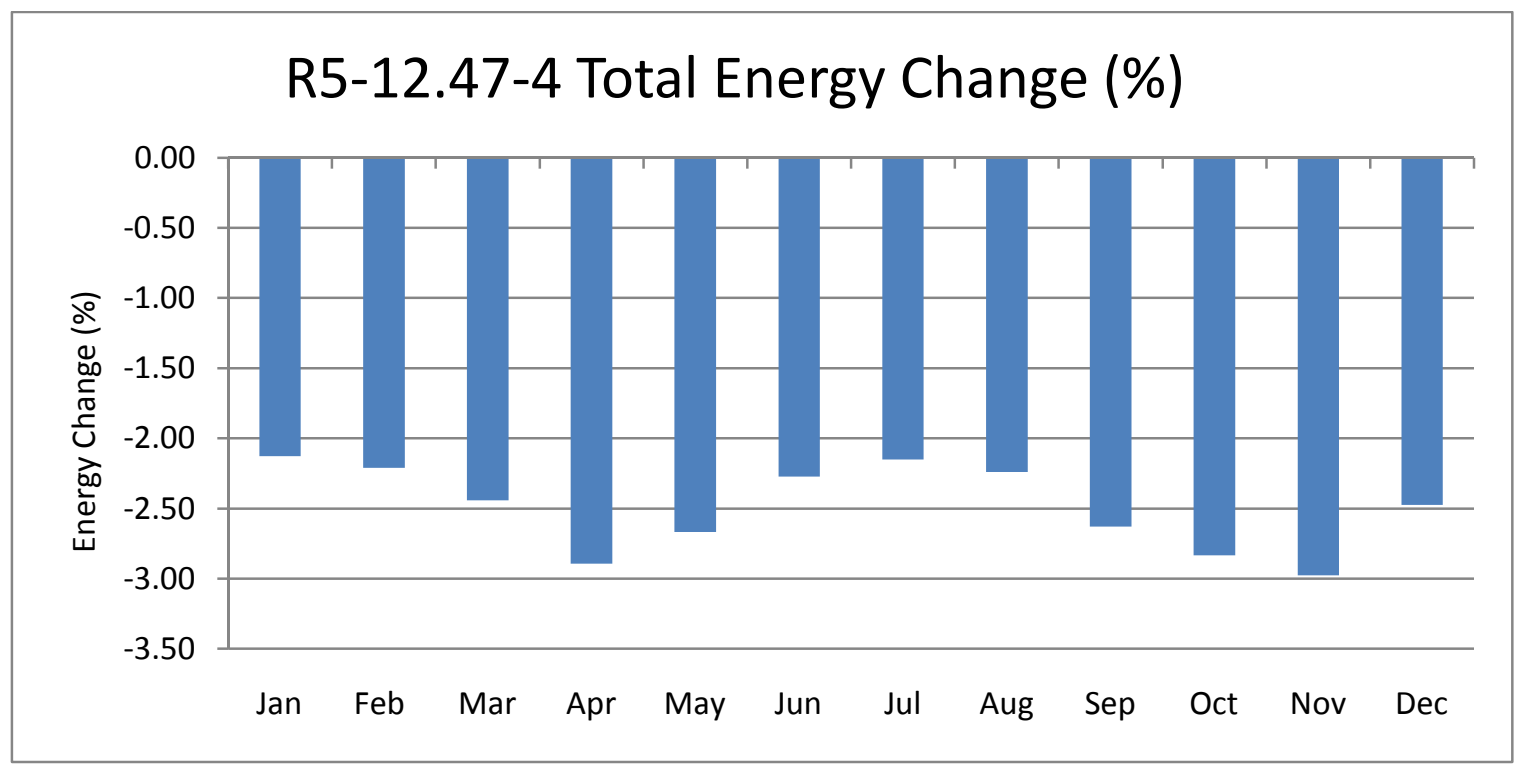

Figure 6.122: R5-12.47-4 Total Energy Change (\%) 


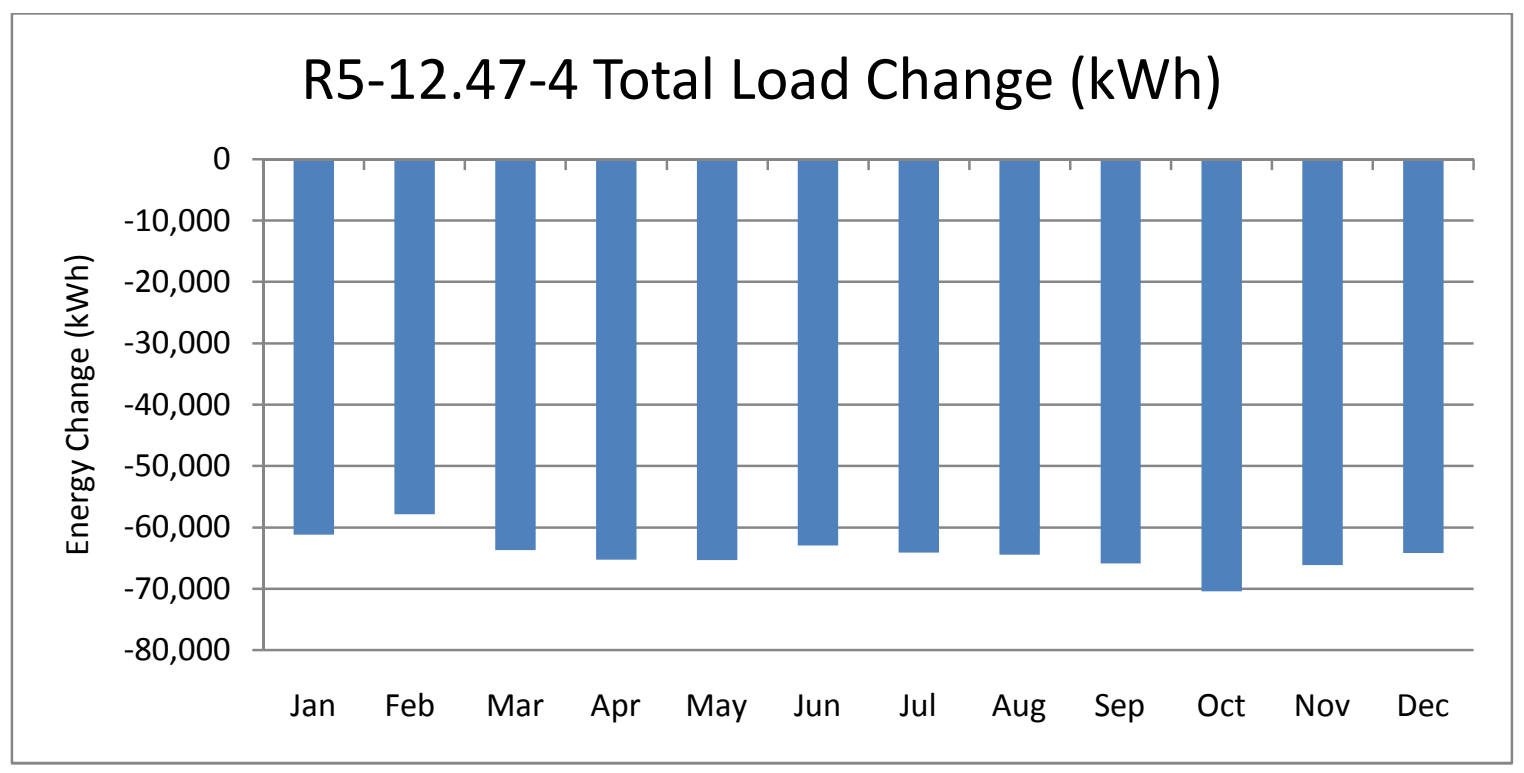

Figure 6.123: R5-12.47-4 Total Load Change (kWh)

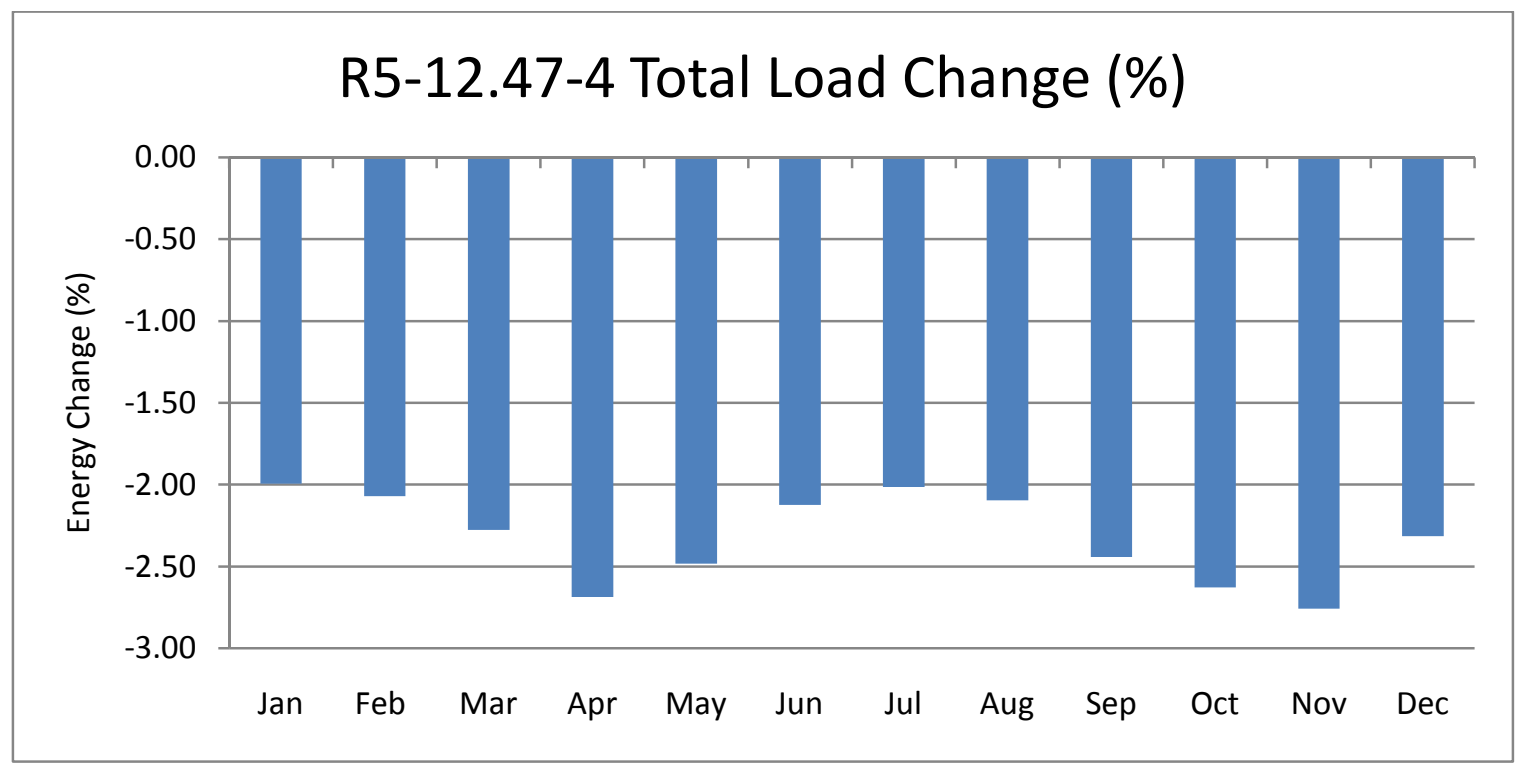

Figure 6.124: R5-12.47-4 Total Load Change (\%) 


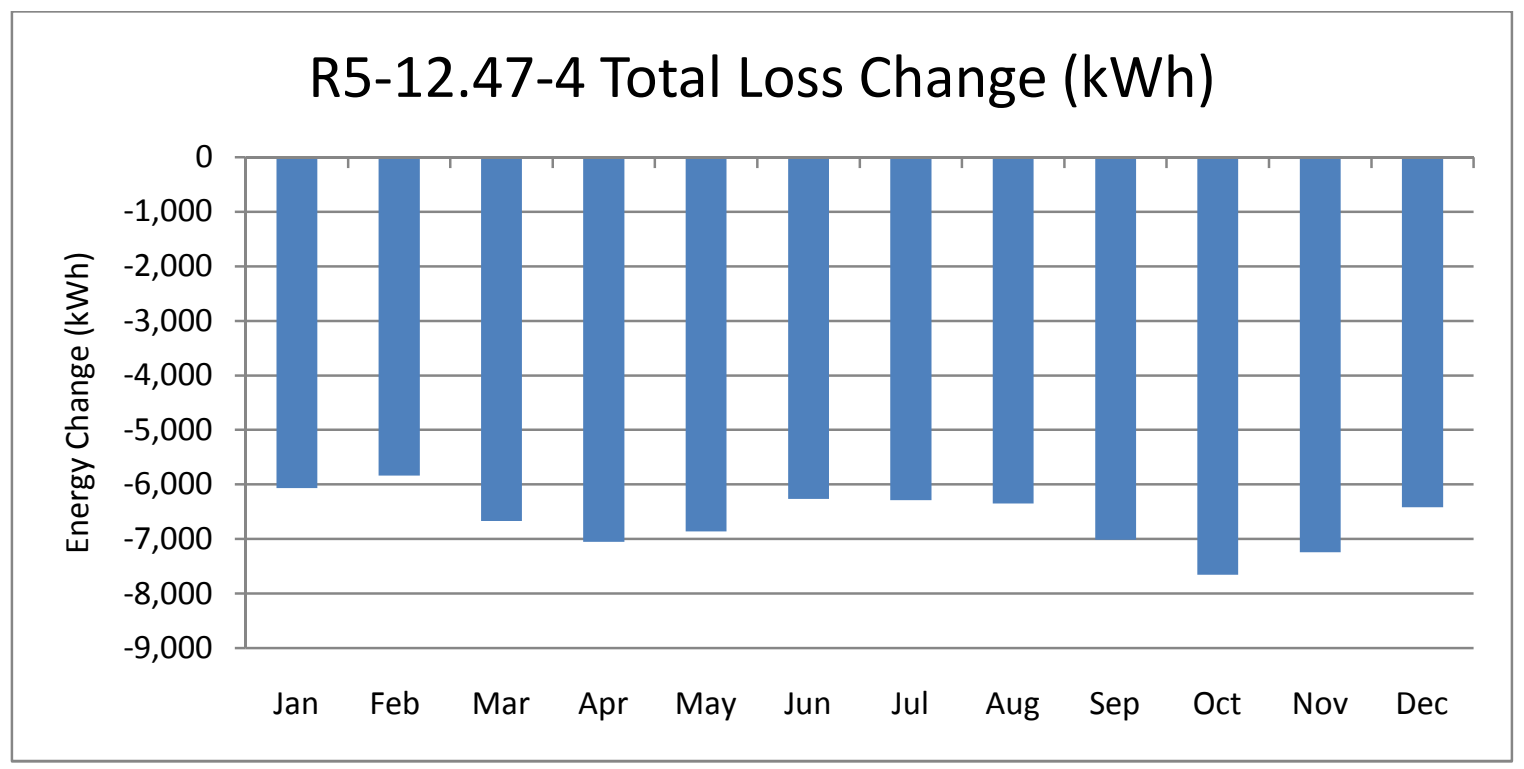

Figure 6.125: R5-12.47-4 Total Loss Change (kWh)

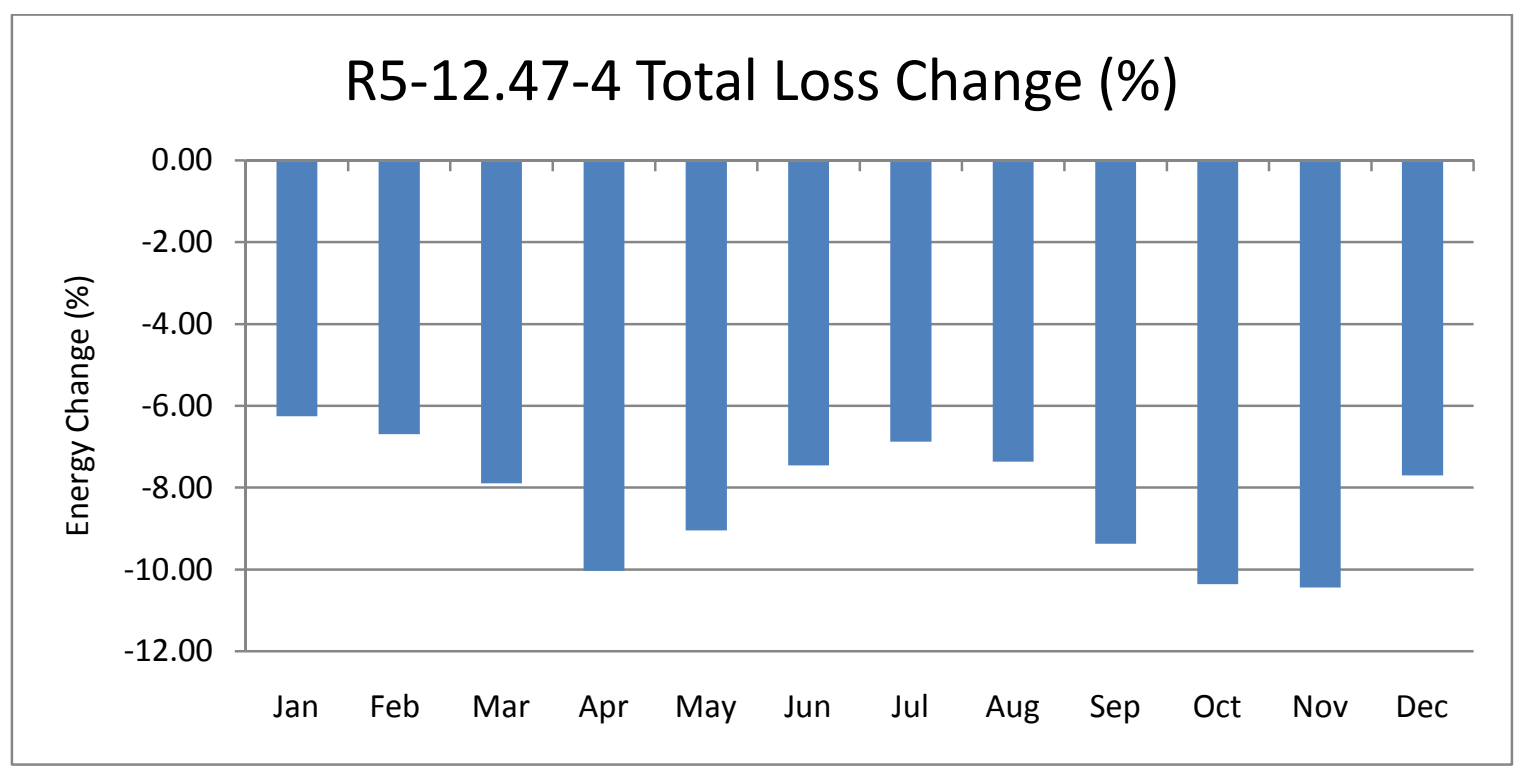

Figure 6.126: R5-12.47-4 Total Loss Change (\%) 


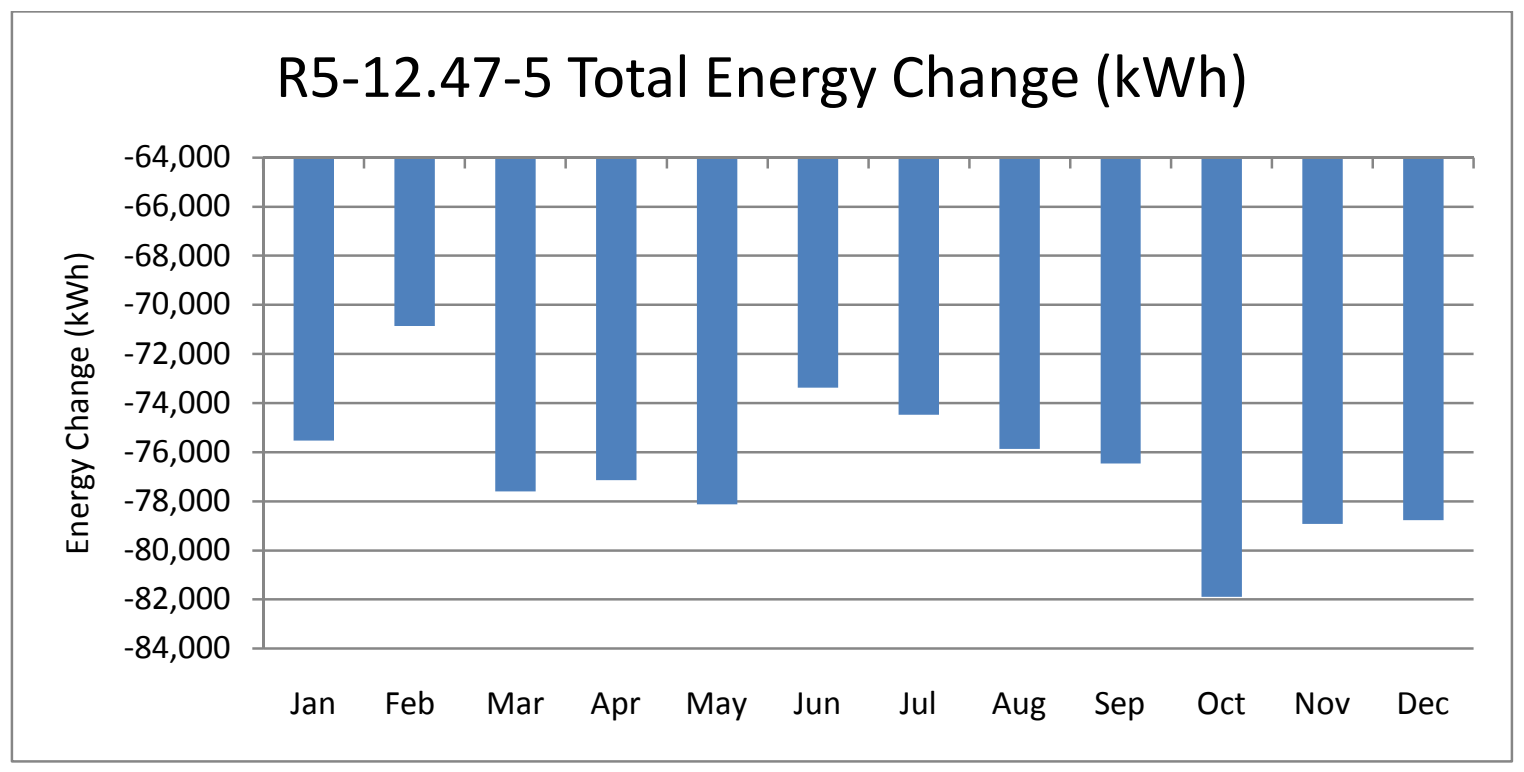

Figure 6.127: R5-12.47-5 Total Energy Change (kWh)

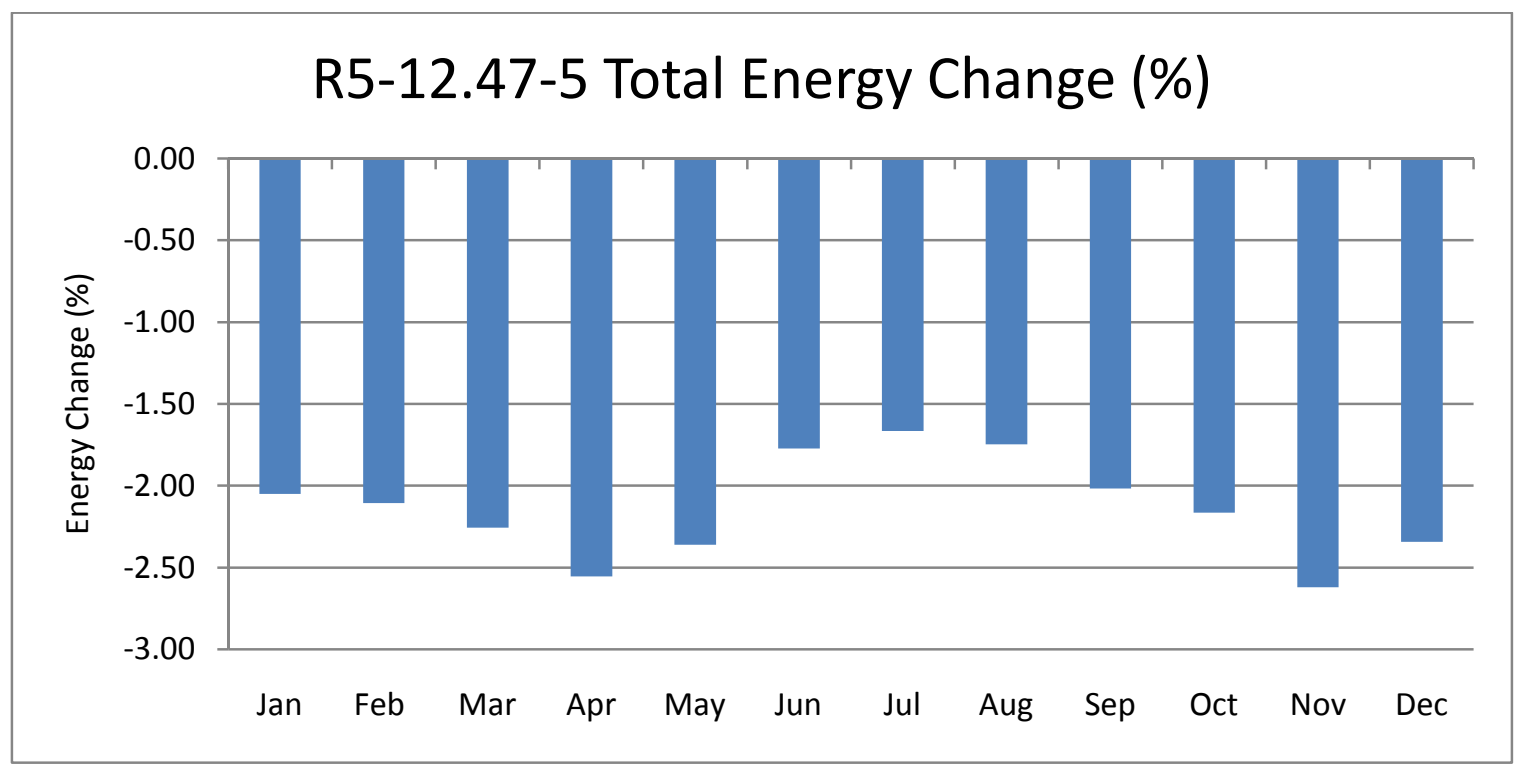

Figure 6.128: R5-12.47-5 Total Energy Change (\%) 


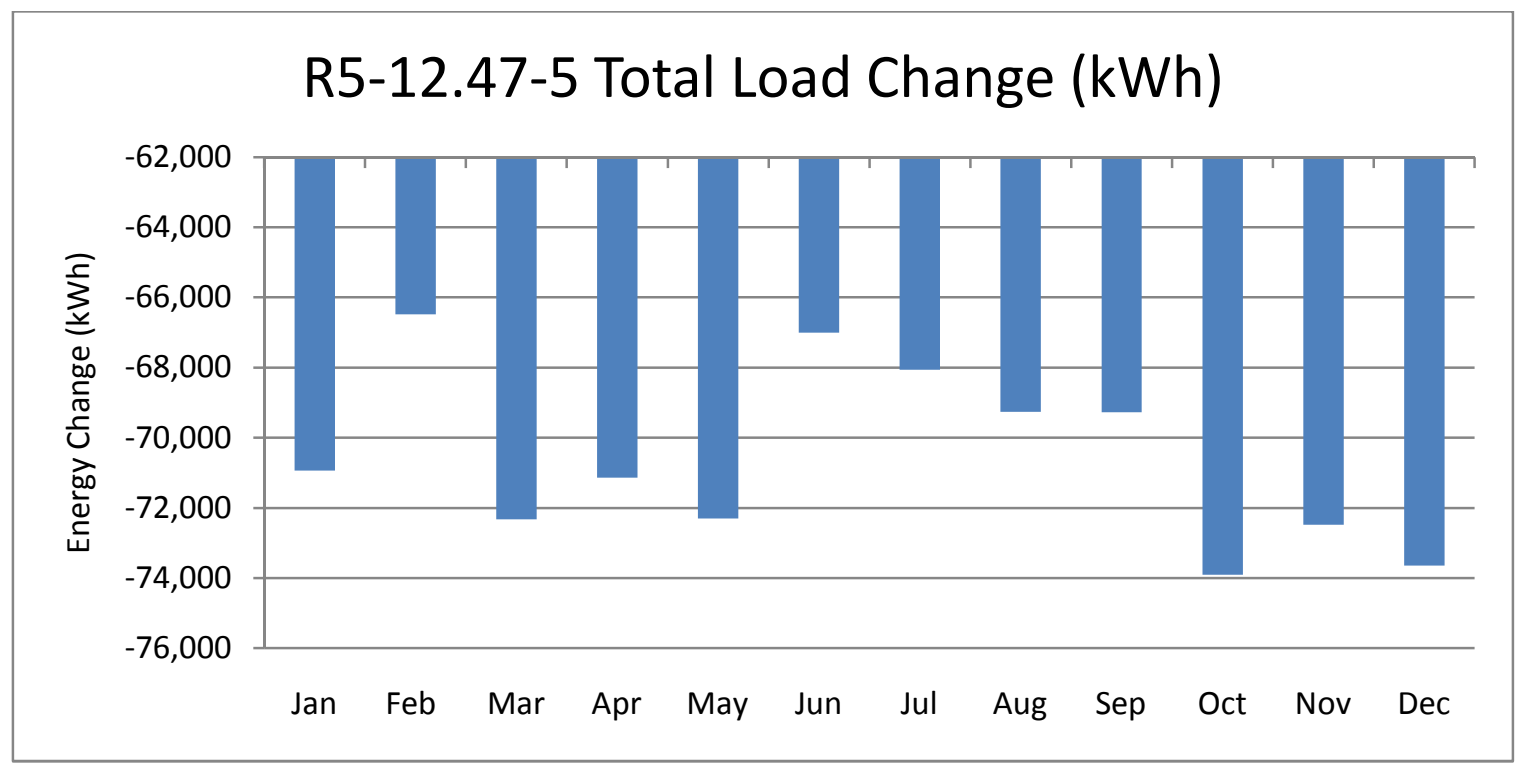

Figure 6.129: R5-12.47-5 Total Load Change (kWh)

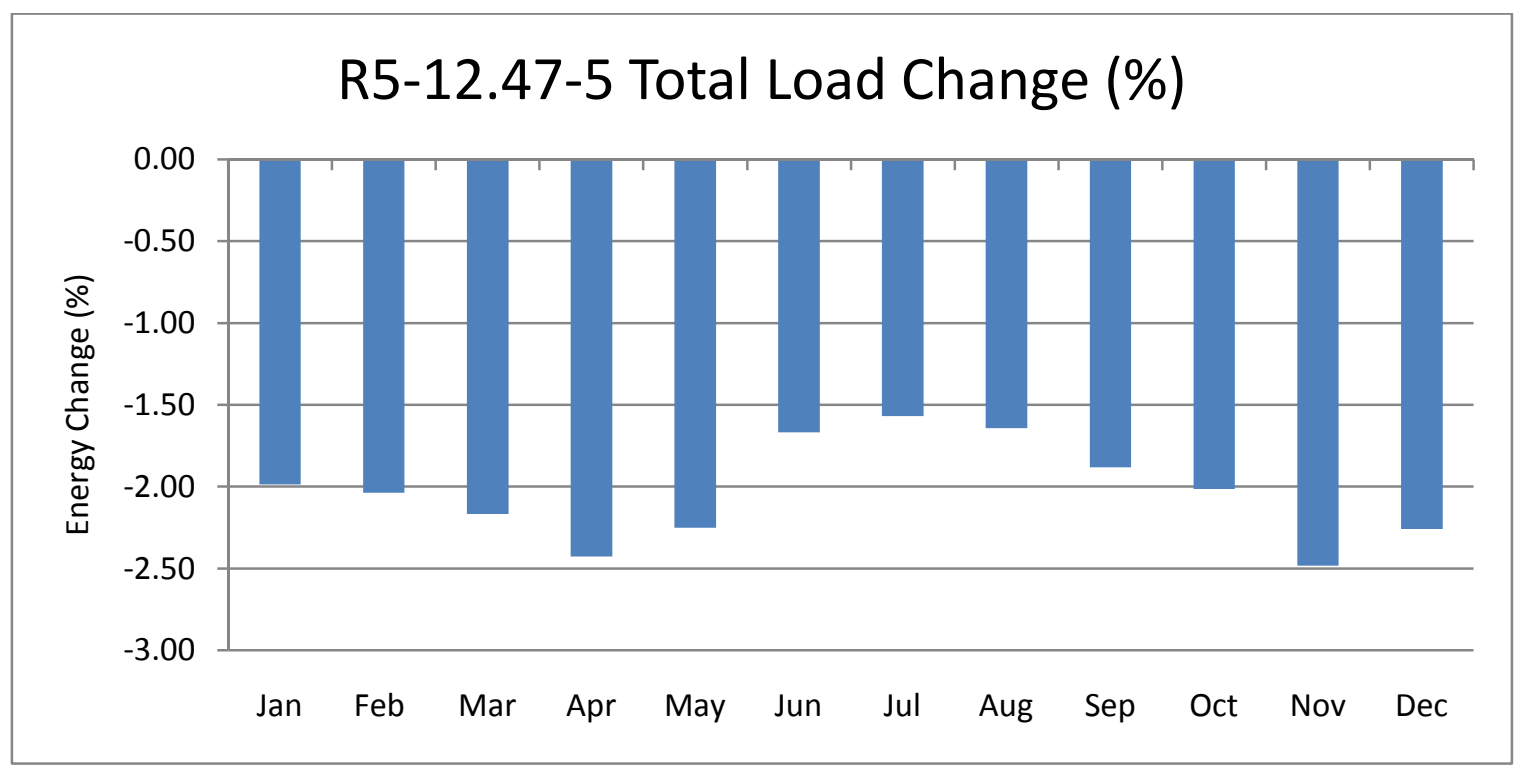

Figure 6.130: R5-12.47-5 Total Load Change (\%) 


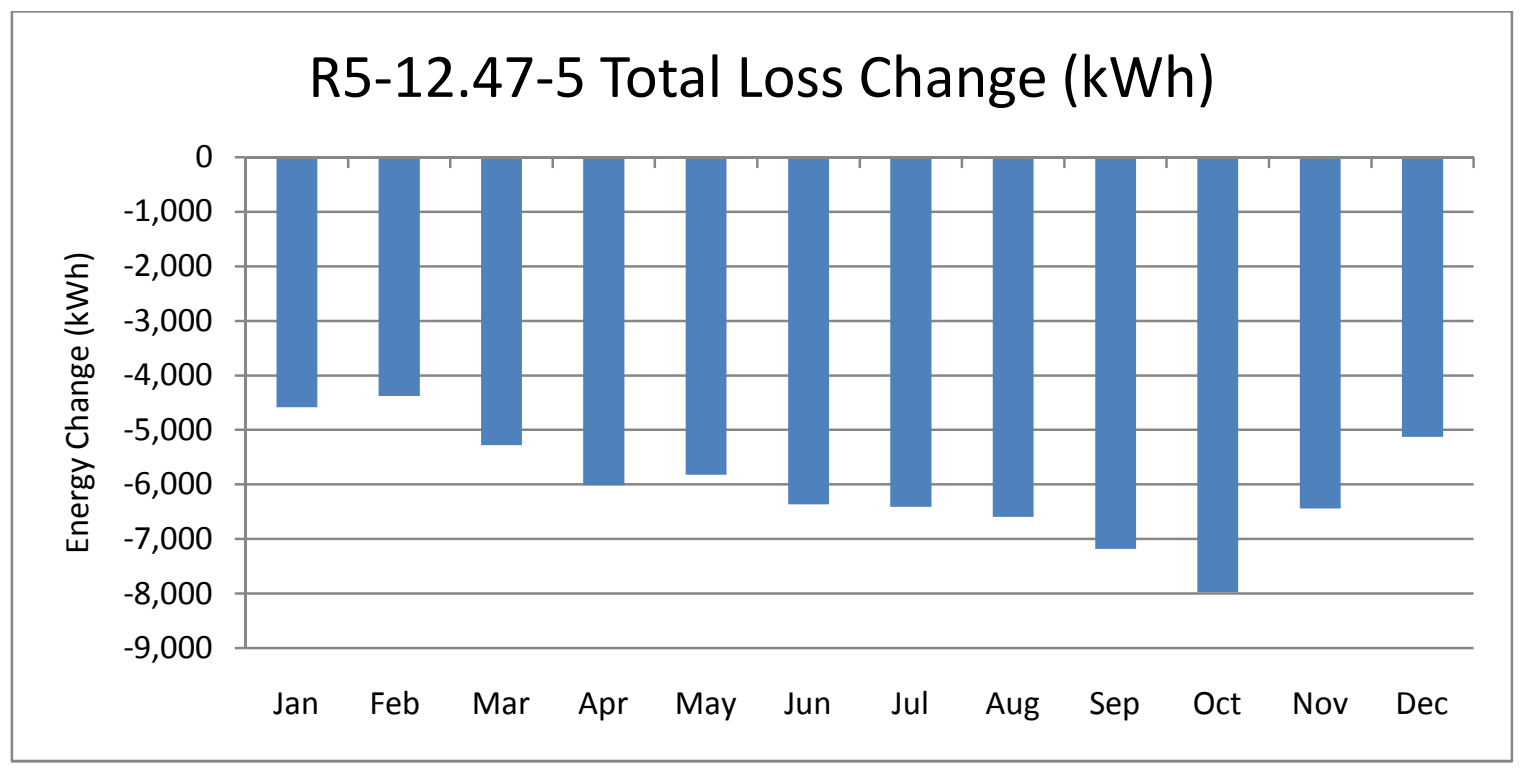

Figure 6.131: R5-12.47-5 Total Loss Change (kWh)

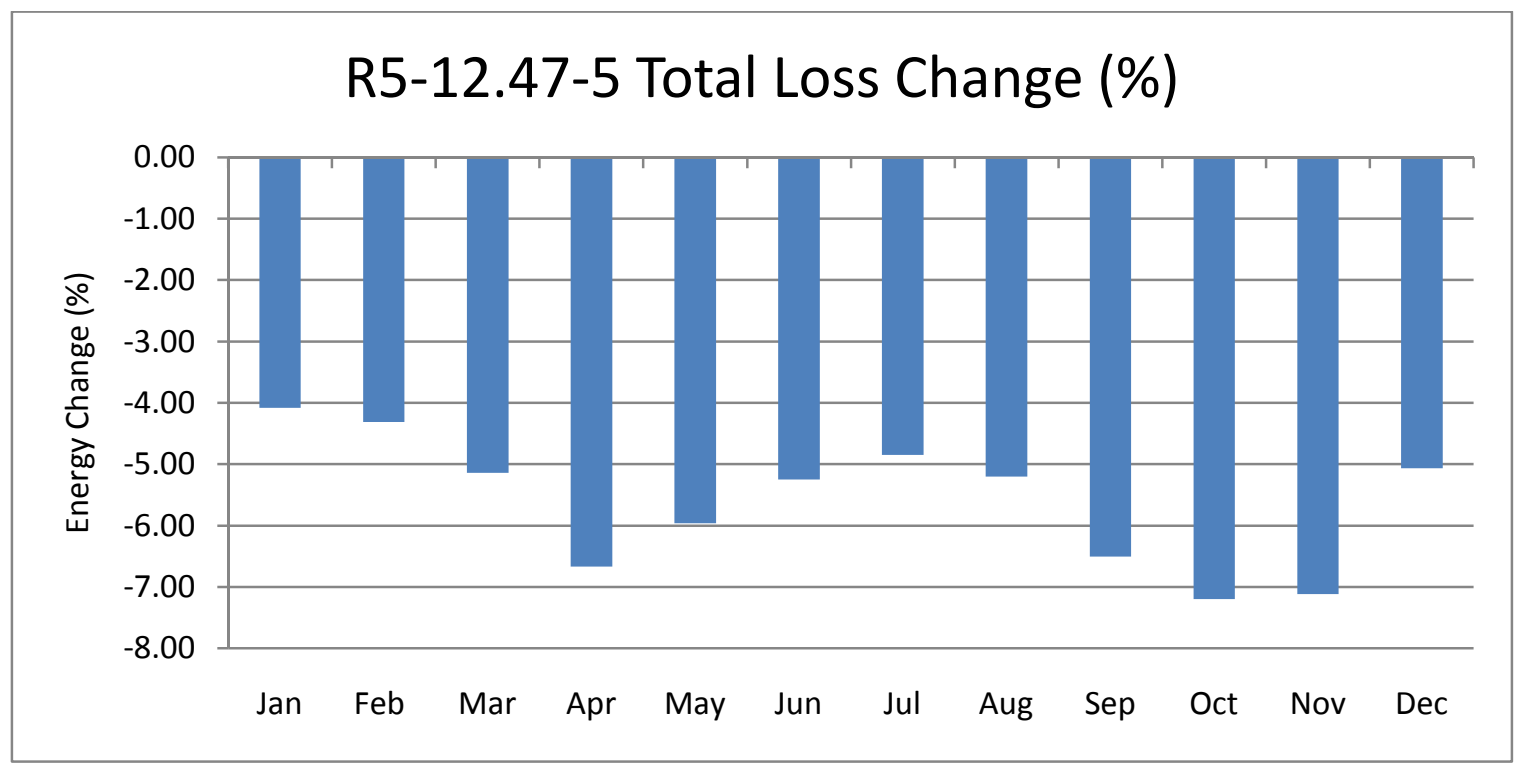

Figure 6.132: R5-12.47-5 Total Loss Change (\%) 


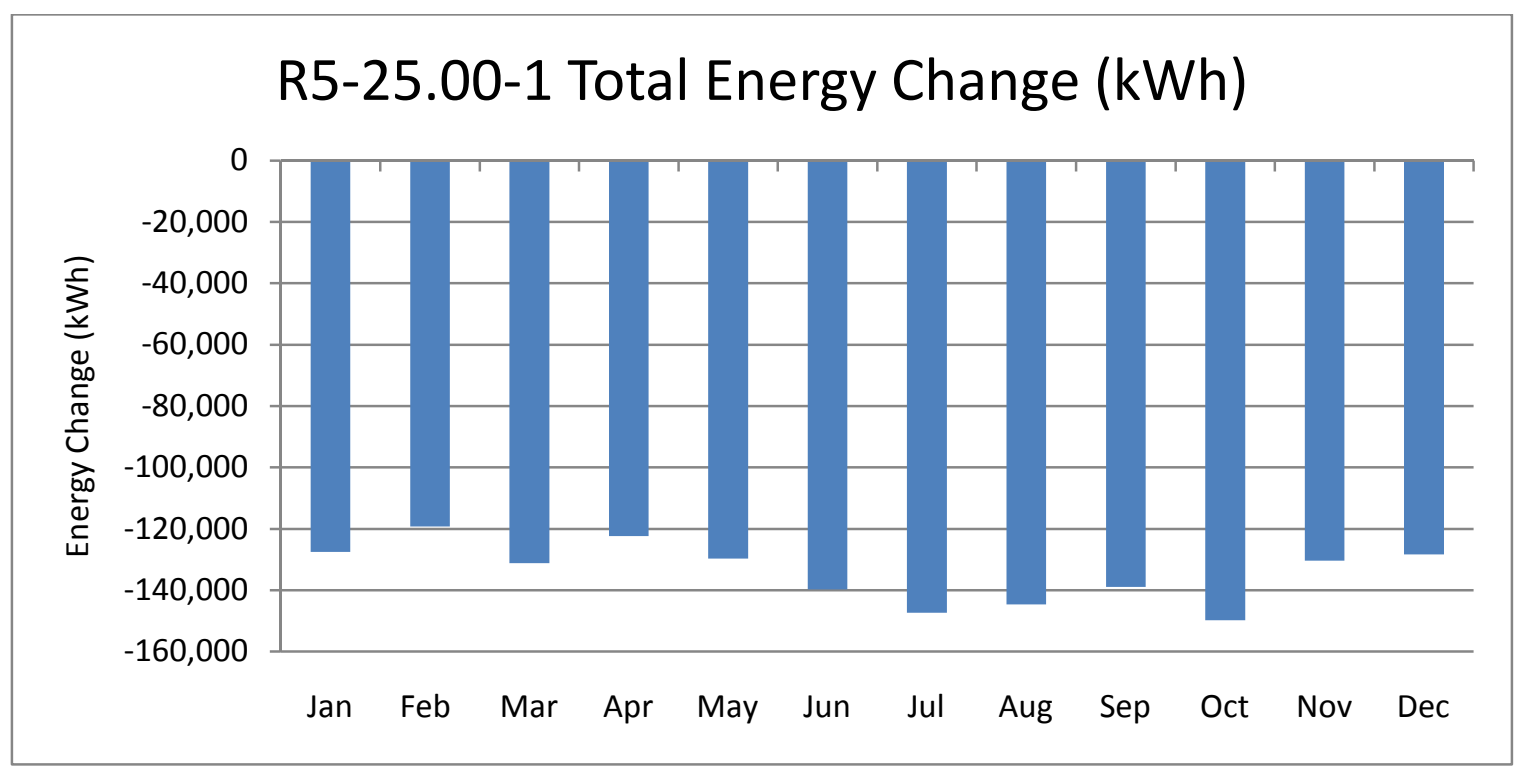

Figure 6.133: R5-25.00-1 Total Energy Change (kWh)

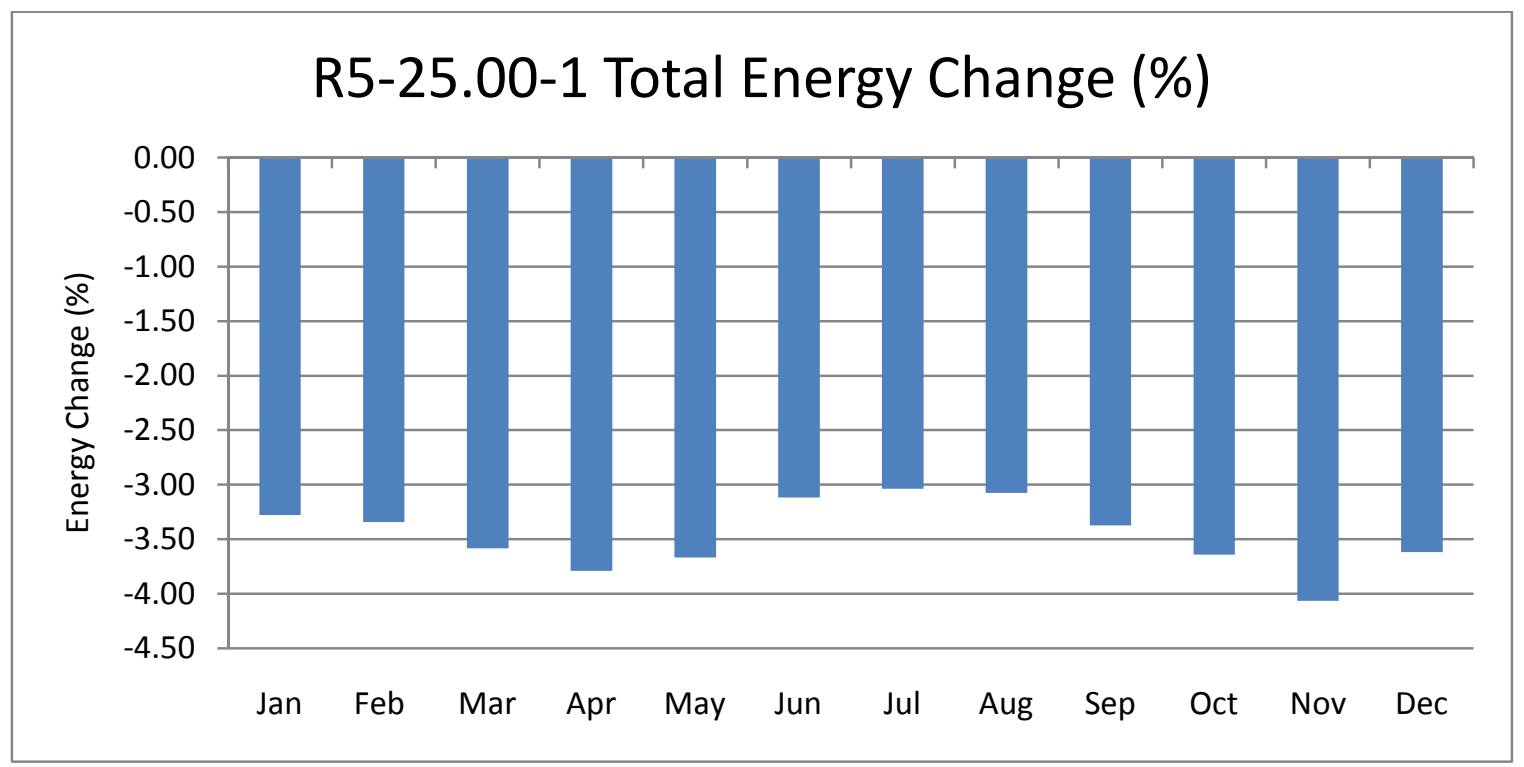

Figure 6.134: R5-25.00-1 Total Energy Change (\%) 


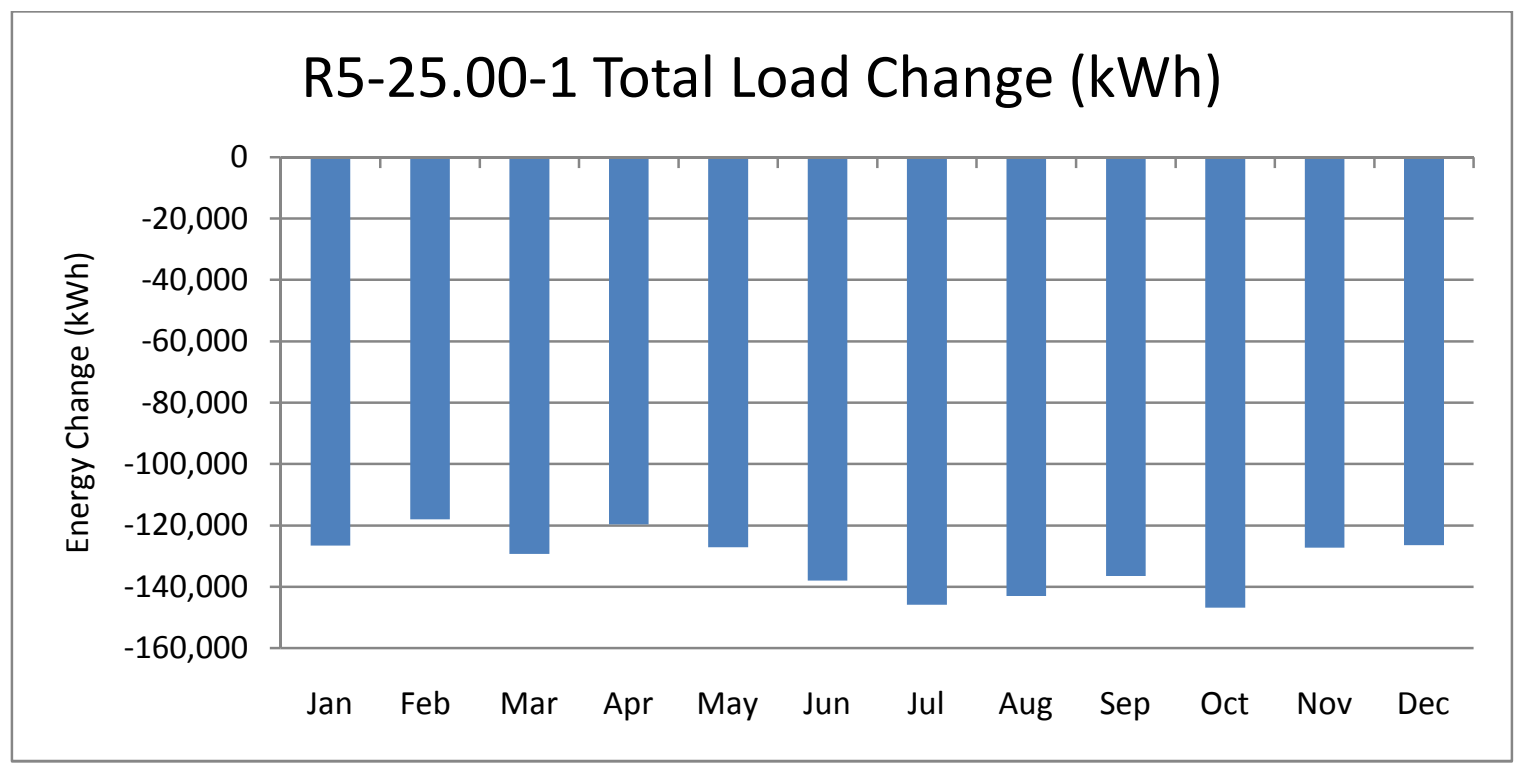

Figure 6.135: R5-25.00-1 Total load Change (kWh)

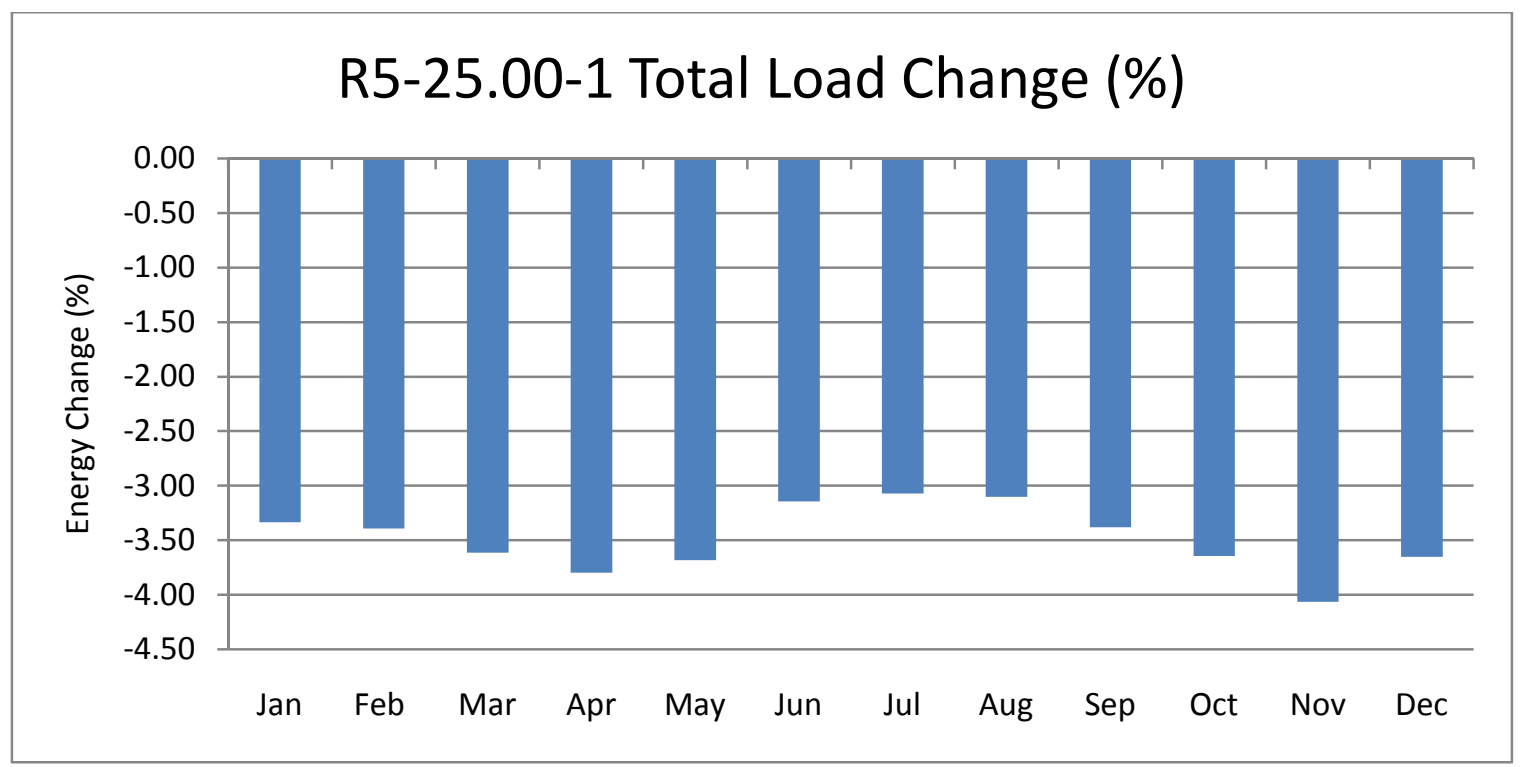

Figure 6.136: R5-25.00-1 Total load Change (\%) 


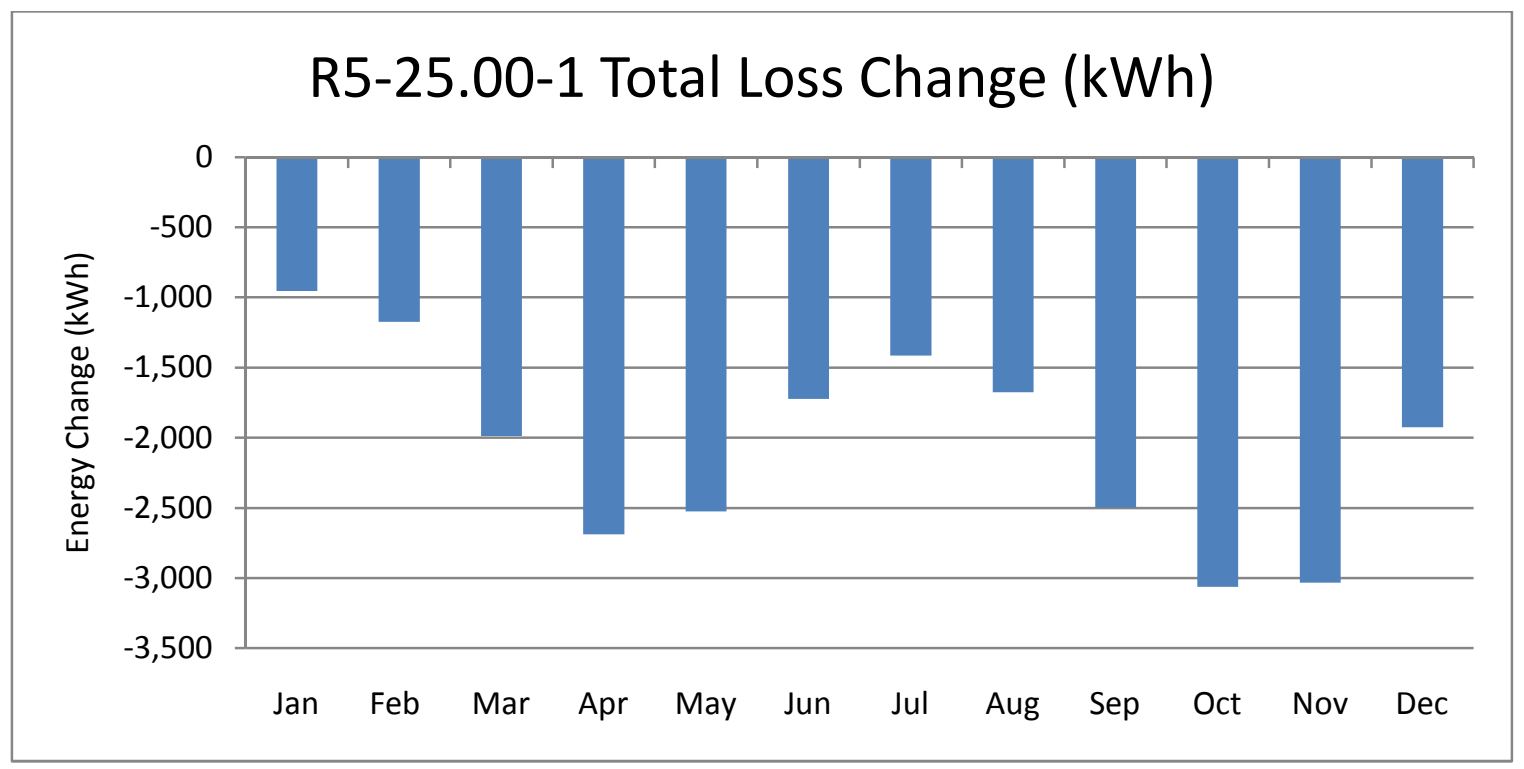

Figure 6.137: R5-25.00-1 Total Loss Change (kWh)

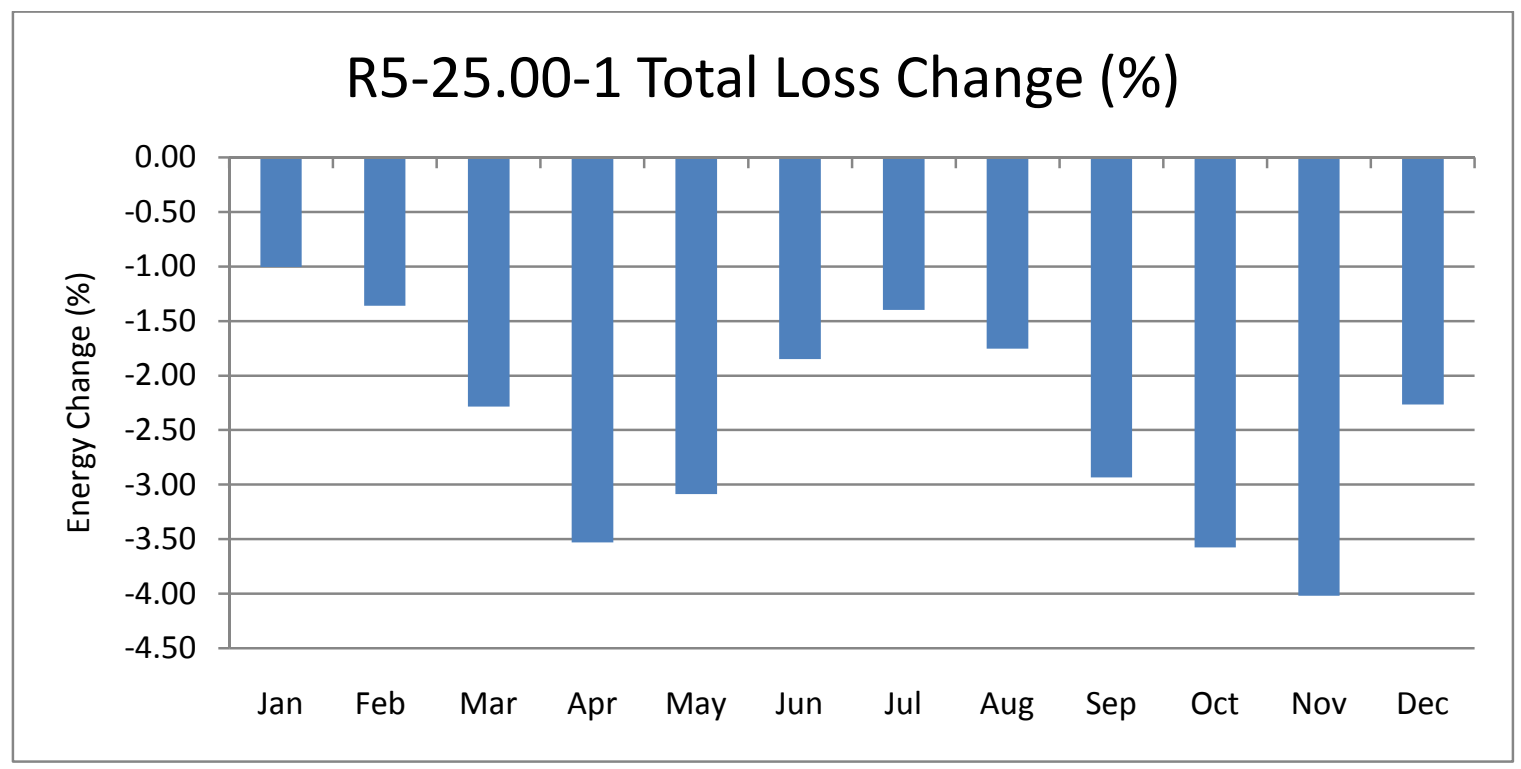

Figure 6.138: R5-25.00-1 Total Loss Change (kWh) 


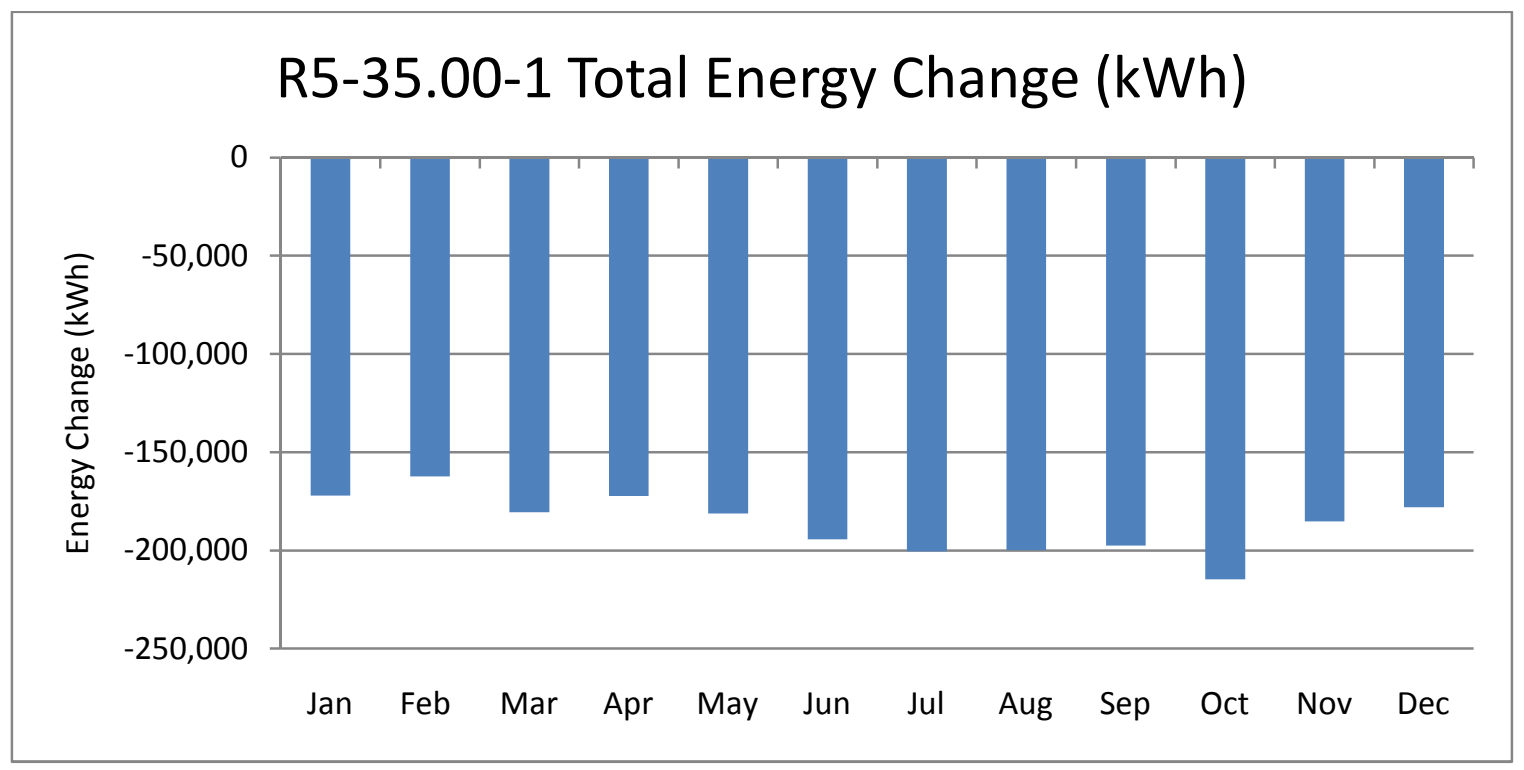

Figure 6.139: R5-35.00-1 Total Energy Change (kWh)

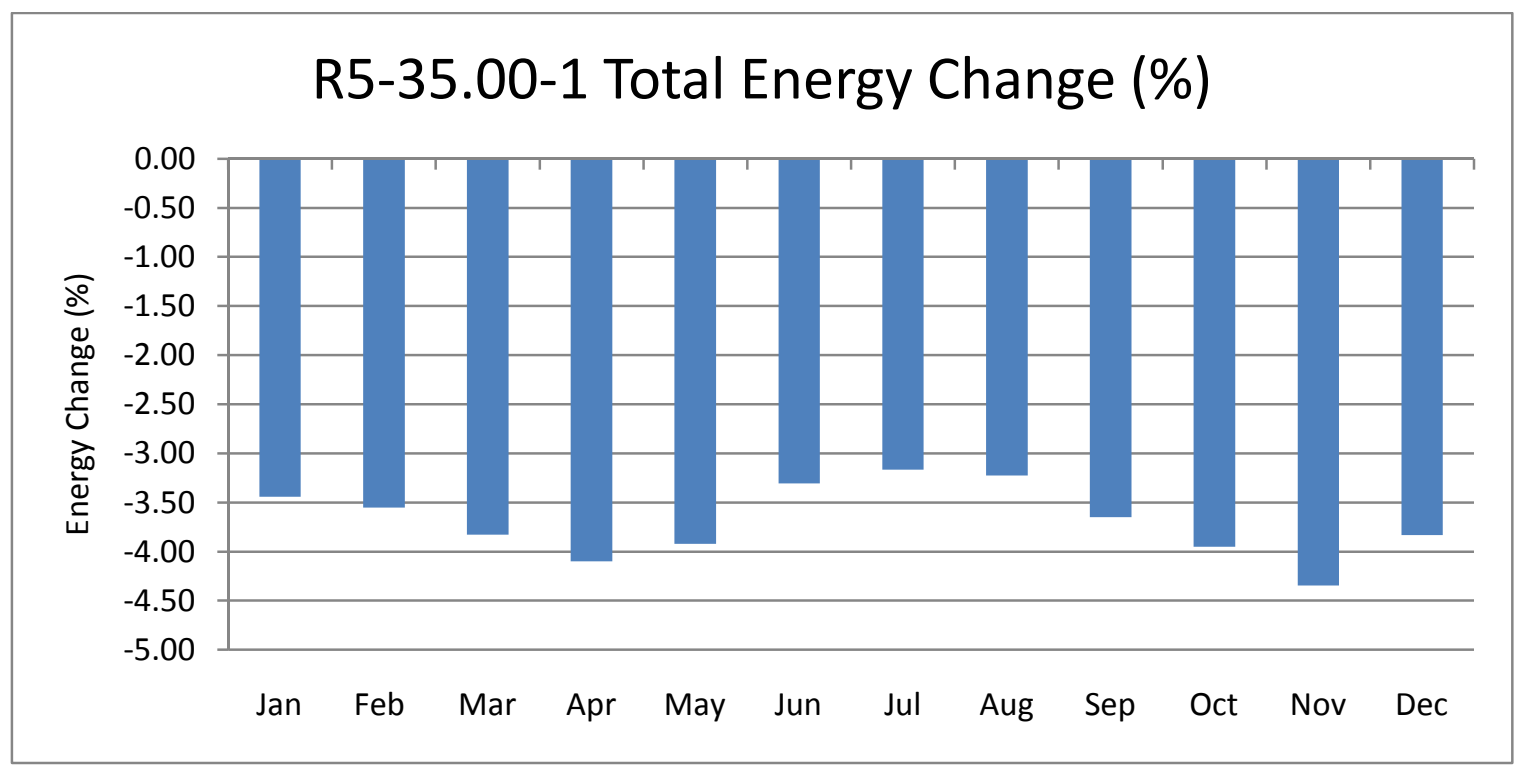

Figure 6.140: R5-35.00-1 Total Energy Change (\%) 


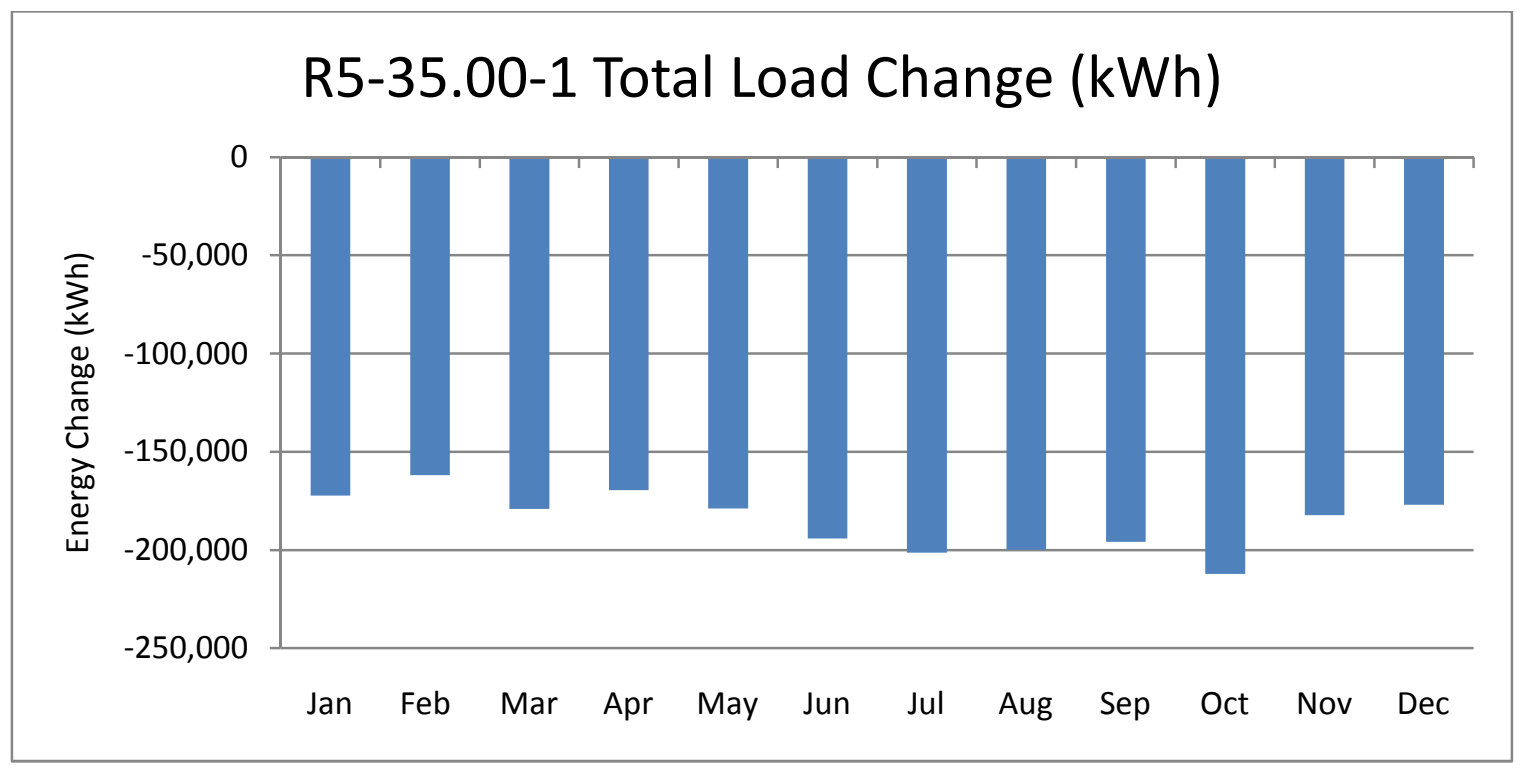

Figure 6.141: R5-35.00-1 Total Load Change (kWh)

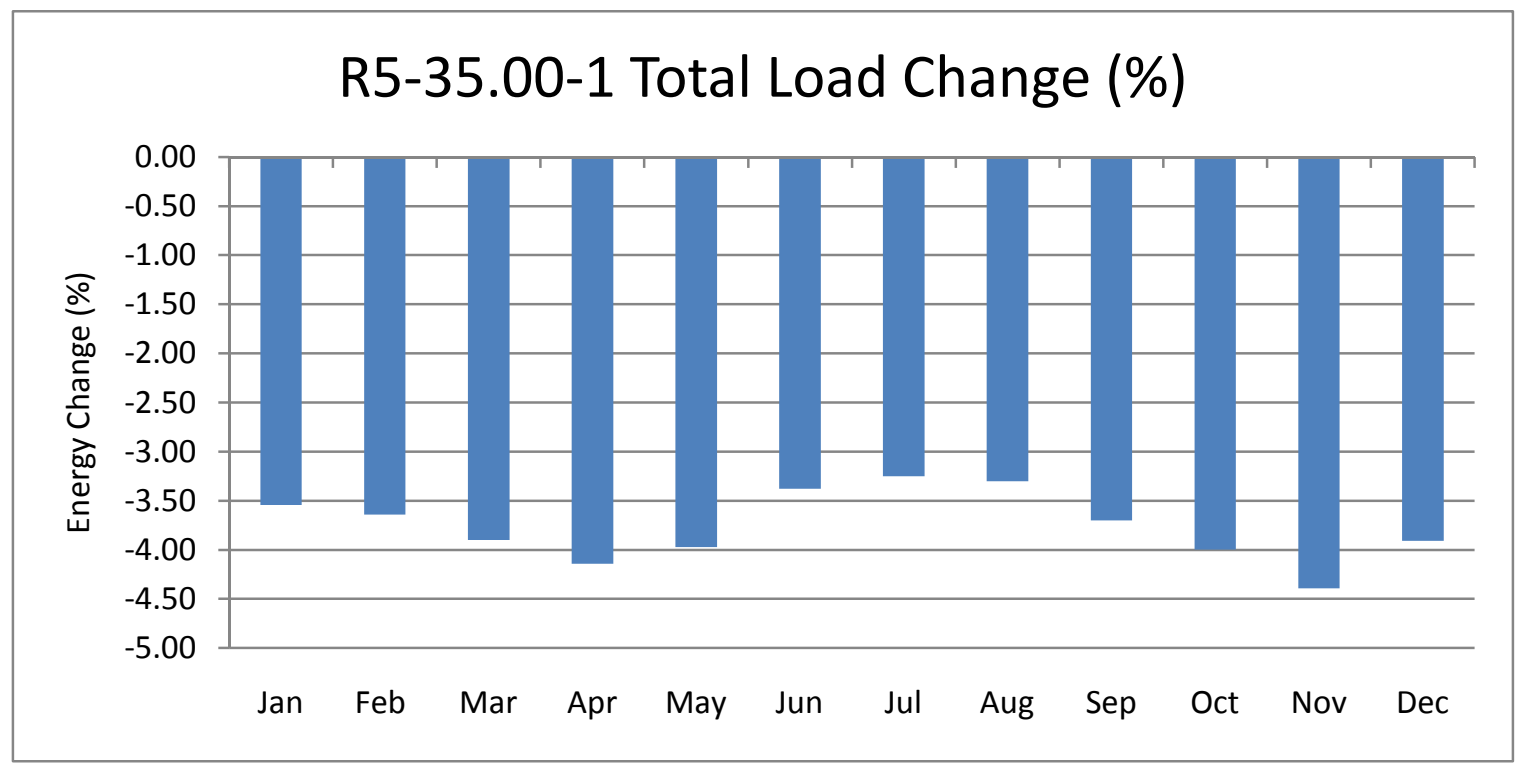

Figure 6.142: R5-35.00-1 Total Load Change (\%) 


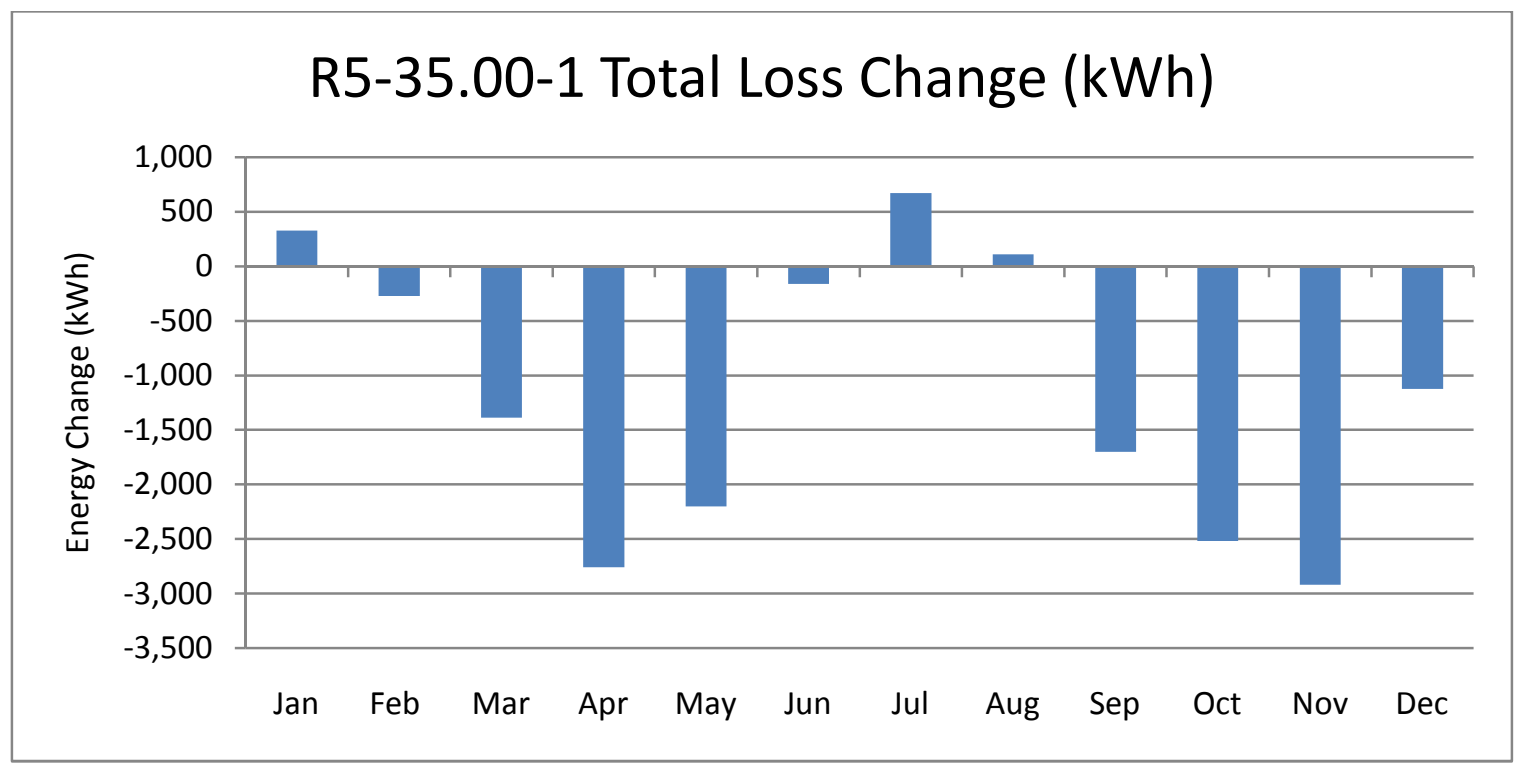

Figure 6.143: R5-35.00-1 Total Loss Change (kWh)

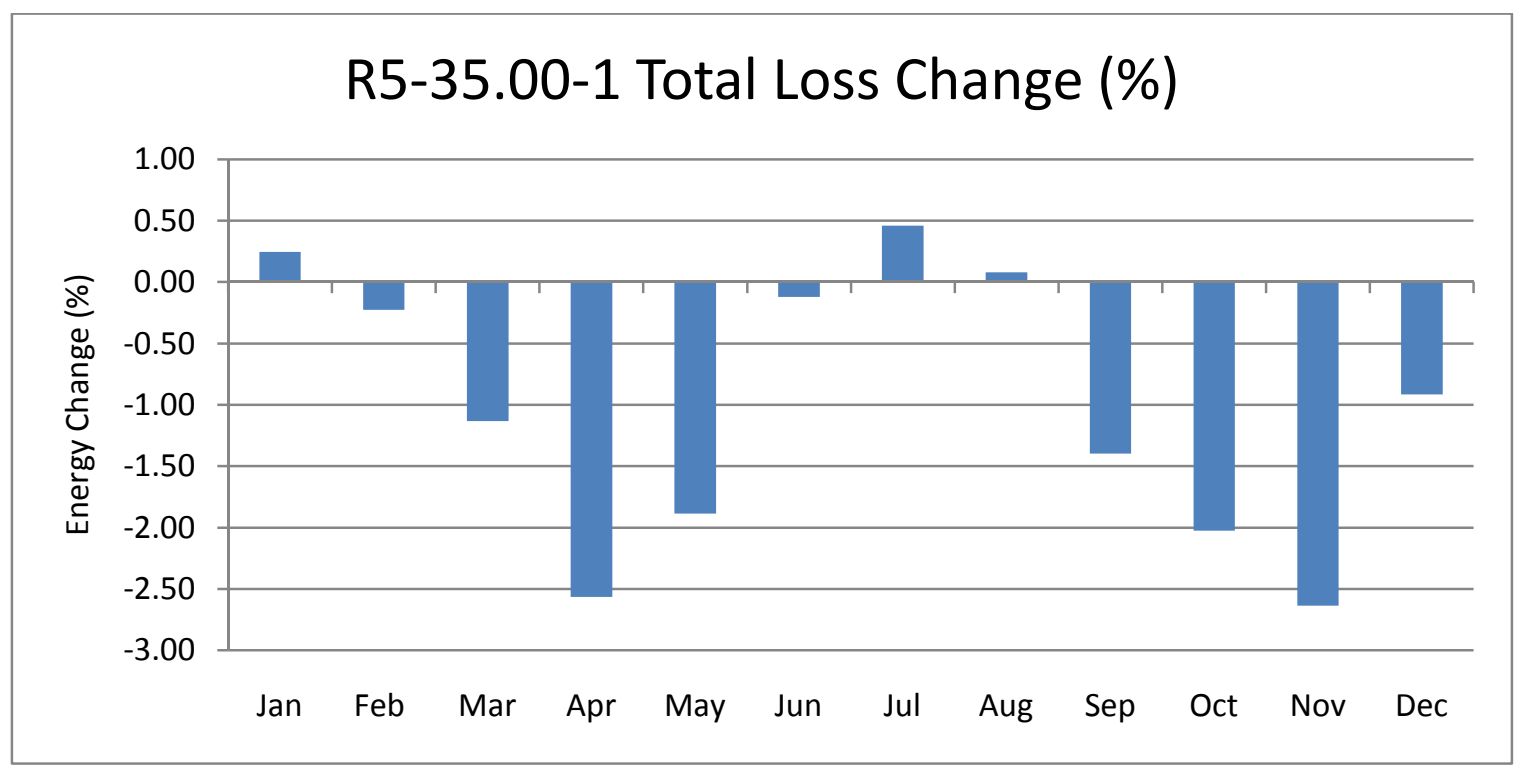

Figure 6.144: R5-35.00-1 Total Loss Change (\%) 


\section{References}

[1] K. Schneider, Y. Chen, D. Chassin, R. Pratt, D. Engel, and S. Thompson, "Modern Grid Initiative Distribution Taxonomy Final Report", PNNL Report 2008.

[2] R. S. Briggs, R. G. Lucus, Z. T. Taylor, "Climate Classification for Building Energy Codes and Standards", ASHRAE Winter Meeting, Chicago, IL, January, 2003

[3] R. Sonderegger, "Dynamic Models of House Heating Based on Equivalent Thermal Parameters", Report PU/CES 57, Doctoral Dissertation 1978, Princeton University, Princeton, New Jersey.

[4] K. Subbarao, "Thermal Parameters for Single and Multizone Buildings and Their Determination from Performance Data", Solar Energy Research Institute, 1981, Golden, Colorado.

[5] N. W. Wilson, B.S. Wagner and W.G. Colborne, "Equivalent Thermal Parameters for an Occupied Gas-Heated House", ASHRAE Transactions, 1985, vol. 91, part 2.

[6] http://www.eia.doe.gov/

[7] http://www.cooperpower.com/Library/Literature/section.asp?ProductLineID=24

[8] M. Dixon, “Autodaptive Volt/VAR Management System” Rural Electric Power Conference, 2001.

[9] B. de Souza and A. de Almeida, "Multiobjective Optimization and Fuzzy Logic Applied to Planning of the Volt/Var Problem in Distributions Systems" IEEE Transactions on Power Systems, Volume pp, Issue 99, pp. 1-1.

[10] A. Sarac and A. Stankovic, "A Robust Algorithm for Volt/VAR Control", IEEE PES Power System Conference and Exposition, 2009.

[11] V. Borozan, M. Baran, and D. Novosel, "Integrated Volt/VAR Control in Distribution Systems", IEEE PES Winter Meeting, 2001.

[12] M. Baran and M. Hsu, "Volt/VAR Control at Distribution Substations" IEEE Transactions on Power Systems, 1999, Volume 14, Issue 1, pp. 312-318.

[13] I. Roytelman, B. Wee, and R. Lugtu, "Volt/CAR Control Algorithm for Modern Distribution management System", IEEE Transactions on Power Systems, 1995, Volume 10, Issue 3, pp. 1454-1460.

[14] S. Auchariyament and S. Sirisumrannukul, $6^{\text {th }}$ International Conference on Electrical Engineering/Electronics, Computer, Telecommunications and Information Technology, 2009.

[15] T. Wilson, "Energy Conservation with Voltage Reduction-Fact or Fantasy", IEEE Rural Electric Power Conference, 2002.

[16] D. Kirshner, "Implementation of Conservation Voltage Reduction at Commonwealth Edison", IEEE Transactions on Power Systems, 1990, Volume 5, Issue 4, pp. 1178-1182.

[17] B. Kennedy and R. Fletcher, "Conservation Voltage Reduction (CVR) at Snohomish County PUD”, IEEE Transactions on Power Systems, 1991, Volume 6, Issue 3, pp 986-998.

[18] D. Lauria, "Conservation Voltage Reduction (CVR) at Northeast Utilities", IEEE Transactions on Power Delivery, 1987, Volume 2, Issue 4, pp. 1186-1191.

[19] B. Scalley and D. Kasten, "The Effects of Distribution Voltage Reduction on Power and Energy Consumption", IEEE Transactions on Education, 1981, Volume 24, Issue 3, pp. 210216. 
[20] S. Lefebvre, G. Gaba, A. Ba, D. Asber, A. Richard, C. Perreault, and D. Chartrand, "Measuring the Efficiency of Voltage Reduction at Hydro-Quebec Distribution", IEEE PES General Meeting, 2008.

[21] J. De Steese, J. Englin, and R. Sands, "Conservation Voltage Reduction potential in the Pacific Northwest", Energy Conversion Engineering Conference, 1990.

[22] V. Dabic, S. Cheong, J. Peralta, and D. Acebedo, "BC Hydro's Experience on Voltage VAR Optimization in Distribution System", IEEE Transmission and Distribution Conference and Exposition, 2010.

[23] General Electric's Integrated Volt Var Control, www.ge.com

[24] Cooper's Integrated Volt/VAR Control, www.cooperpower.com

[25] PCS UtiliData's AdaptiVolt, www.pcsutilidata.com

[26] ABB's Volt/VAR Optimization, www.abb.com 